Universidade de São Paulo

Instituto de Física

\title{
Simulação e validação de perfis de dose e seu uso para estimativa de grandezas dosimétricas para tomografia computadorizada
}

Alejandro Heyner Lopez Gonzales

Orientador: Prof. Dr. Paulo Roberto Costa

Tese de Doutorado apresentada ao Instituto de Física como requisito parcial para a obtenção do título de Doutor em Ciências.

Banca Examinadora:

Prof. Dr. Paulo Roberto Costa (IF-USP)

Prof. Dr. Vito Roberto Vanin (IF-USP)

Prof. Dr. Mauricio Moralles (IPEN)

Prof. Dr. Marcelo Baptista Freitas (Unifesp)

Profa. Dra. Helen Jamil Khoury (UFPE)

São Paulo

2019 


\section{FICHA CATALOGRÁFICA \\ Preparada pelo Serviço de Biblioteca e Informação do Instituto de Física da Universidade de São Paulo}

Lopez Gonzales, Alejandro Heyner

Simulação e validação de perfis de dose e seu uso para estimativa de grandezas dosimétricas para tomografia computadorizada. São Paulo, 2019.

Tese (Doutorado) - Universidade de São Paulo. Instituto de Física. Depto. de Física Nuclear

Orientador: Prof. Dr. Paulo Roberto Costa

Área de Concentração: Física Médica

Unitermos: 1. Física médica; 2. Tomografia computadorizada por raios X; 3 . Dosimetria; 4. Método de Monte Carlo.

USP/IF/SBI-094/2019 


\title{
University of São Paulo
}

Physics Institute

\section{Simulation and validation of dose profiles and their use to estimate dosimetric quantities for Computed Tomography}

\author{
Alejandro Heyner Lopez Gonzales
}

Supervisor: Prof. Dr. Paulo Roberto Costa

Thesis submitted to the Physics Institute of the University of São Paulo in partial fulfillment of the requirements for the degree of Doctor in Science.

Examining Committee:

Prof. Dr. Paulo Roberto Costa (IF-USP)

Prof. Dr. Vito Roberto Vanin (IF-USP)

Prof. Dr. Mauricio Moralles (IPEN)

Prof. Dr. Marcelo Baptista Freitas (Unifesp)

Profa. Dra. Helen Jamil Khoury (UFPE)

São Paulo

2019 
To my Family. 


\section{ACKNOWLEDGMENTS}

This work could never exist if my parents Rosa and Alejandro did not support my passion for Physics and believe I could make it right.

I want to thank my beautiful family to help me to be where and who I am: my sisters and brother, my aunts and uncles.

I want to thank my advisor Paulo for accepting a foreigner as his student and give me support, time, and to have patience with me. It was an incredible experience.

I want to thank my colleagues Denise and Nancy to help me with this work, to support me in the experimental measurements and dosimeter reading, respectively.

I want to thank José Maria and Josep Sempau to support me with the Monte Carlo modelling using the PENELOPE/penEasy package.

I want to thank Ricardo Terini to provide me the ribbon OSL dosimeters for dose profile measurements.

Special thanks go to my sister Janira, my colleagues: Josilene, Ana, and Louise who correct the thesis. However, for errors that remain, I am alone responsible for and apologize in advance.

I want to thank the whole group of the Radiation Dosimetry and Medical Physics who supported my work and the good coffee times.

I want to thank my professors of the Medical Physics and Radiation Dosimetry Group Elisabeth, Emico, Ricardo Terini, and Ana Regina who made me feel welcome.

I want to thank João Terassi for the designs performed.

I want to thank InRaD for allowing me to take access to the CT machines.

I want to thank Comissão Nacional de Energia Nuclear (CNEN) for financial support of the Doctoral Scholarship and the CNPq/FAPESP for funding of the project by INCT - Metrology of ionizing radiation in medicine [grant number 2008/57863-2]. 
"Listen, damn it, we will win"

James Hetfield 


\title{
Simulation and Validation of Dose Profiles and their use to estimate dosimetric quantities for Computed Tomography
}

\begin{abstract}
Computed Tomography (CT) has become a reference medical imaging technique with number of procedures growing over the years. This fact is mainly due to the rapid evolution of CT technology that means the utilization of the devices in a number of applications. Since a CT procedure implies dose delivered to the patient, it is a concern to track the radiation emission by a CT device. Efforts aiming to fill the gap about the capability of the traditional Computed Tomography Dose Index (CTDI) methodology to estimate the radiation emission characteristics of a current CT device have been performed. An analytical approach about the description of the dose distribution in cylindrical phantoms subdued to clinical CT scanning has been developed. This analytical approach helps to have a better understanding of the physical processes governing the dose deposition in a cylindrical phantom. The analytic approach predicts with sufficient accuracy the dose profile and the cumulated dose profile from single and multiple CT scans, respectively, along the central and peripheral axes of a cylindrical phantom. Moreover, from the single or multiple dose profiles, it is possible to derive more accurate dose descriptors for the current CT examinations than the traditional CTDI 100 metric.

In this work, a Monte Carlo (MC) modelling of the helical CT scan mode was performed aiming to compute single and cumulated dose profiles. The radiation emission characteristics of the multiple-detector CT (MDCT) scanners, GE Discovery CT750HD, and GE Lightspeed Ultra were modelled and used to assess dose deposition in PMMA cylindrical phantoms of 16 and $32 \mathrm{~cm}$ diameters. To model the helical procedure and the radiation transport of photons and electrons in the phantom material, the PENELOPE/penEasy package was used. The target self-attenuation, focal spot angle, and fan-beam geometry are incorporated. Body helical protocol with pitch values of $0.516,0.984$, and 1.375, nominal beam width, $n T=40 \mathrm{~mm}$, and the head helical protocol with pitch values of $0.531,0.969$, and 1.375 , and nominal beam width $n T=20 \mathrm{~mm}$ were chosen to be modelled for X-ray spectra corresponding to $80,100,120$, and $140 \mathrm{kV}$. The analytical formulation for the single dose profiles and experimental measurements of single and cumulated dose profiles were used to validate the MC modelling. The experimental dose profiles were measured using Optically Stimulated Luminescence (OSL) dosimeters in the form of ribbons. Also, the experimental values of the $C T D I_{100}$ were used to calibrate the simulated single and cumulative profiles. The match of the simulated dose profiles with the reference data reflects the correct modelling of the target self-attenuation and the radiation transport in the phantom material reflected in the tails of the dose profiles. From the calibrated cumulative dose profiles, metrics describing the CT dose characteristics were derived, such as Dose Line Integral $(D L I)$, Equilibrium Dose $\left(D_{e q}\right)$, pitchxEquilibrium Dose product $\left(p x D_{e q}\right)$, Approach to Equilibrium function $(H(L))$, and Equilibrium Scanning Length $\left(L_{e q}\right)$. A comprehensive data for the aforementioned metrics are obtained. $L_{e q}$ agrees with data from the literature along the central axis. The efficiency of the $C T D I_{100}$ to assess the product $p x D_{e q}$ were $80.8 \%$, and $87.8 \%$ for head phantom, and $56.5 \%$, and $86.2 \%$ for the body phantom along the central and peripheral axes for $120 \mathrm{kV}$.
\end{abstract}

Keywords: dose profiles, CT dose descriptors, MC modelling, CT helical scan mode 


\title{
Simulação e Validação dos Perfis de Dose e seu uso para estimativa de grandezas dosimétricas para Tomografia Computadorizada
}

Resumo

\begin{abstract}
A tomografia computadorizada (TC) tornou-se uma técnica de imagem médica de referência, com um número de procedimentos crescendo ao longo dos anos devido principalmente à rápida evolução da tecnologia CT. Como um procedimento de TC implica a dose entregue ao paciente, é uma preocupação rastrear a emissão de radiação por um dispositivo de TC. Esforços visando preencher a lacuna sobre a capacidade da metodologia tradicional do Índice de Dose por Tomografia Computadorizada (CTDI) para estimar as características de emissão de radiação de um dispositivo de TC atual foram realizados. Uma abordagem analítica sobre a descrição da distribuição da dose em simuladores cilíndricos submetidos a procedimentos de CT clínicos foi desenvolvida. Essa abordagem analítica ajuda a entender melhor os processos físicos que governam a deposição de doses em um simulador cilíndrico. A abordagem analítica prediz com precisão suficiente o perfil de dose e o perfil de dose acumulado a partir de uma única e múltipla tomografia computadorizada, respectivamente, ao longo dos eixos central e periférico de um simulador cilíndrico. Além disso, a partir dos perfis de dose única ou múltipla, é possível derivar descritores de dose mais precisos para os exames de TC atuais do que a métrica tradicional CTDI $I_{100}$.

Neste trabalho, uma modelagem de Monte Carlo (MC) do modo de CT helicoidal foi realizada com o objetivo de calcular perfis de dose acumulados. As características de emissão de radiação dos scanners CT com detector múltiplo (MDCT), GE Discovery CT750HD e GE Lightspeed Ultra foram modeladas e usadas para avaliar a deposição de dose em phantoms cilíndricos de PMMA de 16 e $32 \mathrm{~cm}$ de diâmetro. Para modelar o procedimento helicoidal e o transporte de radiação de fótons e elétrons no material do simulador, foi utilizado o pacote PENELOPE/penEasy. A auto-atenuação do alvo, o ângulo do ponto focal e a geometria do feixe são incorporados. Foram escolhidos um protocolo helicoidal de corpo com valores de pitch de 0,516, 0,984 e 1,375, largura nominal do feixe, $n T=40 \mathrm{~mm}$, e um protocolo helicoidal da cabeça com valores de pitch de 0,531, 0,969 e 1,375 e largura nominal do feixe $n T=20 \mathrm{~mm}$ para serem modelados utilizando espectros de raios $X$ correspondentes a 80, 100, 120 e $140 \mathrm{kV}$. Os perfis de dose experimentais foram medidos usando dosímetros de Luminescência Opticamente Estimulada (OSL) na forma de fitas. Além disso, os valores experimentais do $C T D I_{100}$ foram usados para calibrar os perfis acumulativos simulados. A correspondência dos perfis de dose simulados com os dados de referência mostra a modelagem correta da auto atenuação do alvo e do transporte de radiação no material do phantom refletidos nas caudas dos perfis de dose. A partir dos perfis de dose cumulativa calibrada, foram derivadas métricas que descrevem as características da dose de TC, como Integral linear da dose $(D L I)$, Dose de equilíbrio $\left(D_{e q}\right)$, produto de Dose de equilíbrio e pitch $\left(p x D_{e q}\right)$, função de equilíbrio $(H(L))$ e Varredura de equilíbrio $\left(L_{e q}\right)$. São obtidos dados abrangentes para as métricas mencionadas acima. $L_{e q}$ concorda com os dados da literatura ao longo do eixo central. A eficiência do $C T D I_{100}$ para avaliar o produto $p x D_{e q}$ foi de $80,8 \%$ e $87,8 \%$ para o phantom de cabeça e $56,5 \%$ e $86,2 \%$ para o phantom de corpo ao longo dos eixos central e periférico para $120 \mathrm{kV}$.

Palavras-chave: perfis de dose, descritores de dose de TC, modelagem de MC, modo de varredura helicoidal de TC.
\end{abstract}




\section{List of Figures}

Figure 1. Schematic illustration to obtain attenuation profiles, $\mathrm{p} \gamma 1$ and $\mathrm{p} \gamma 2$, at two angular positions, $\gamma 1$ and $\gamma 2$, for a system composed by three squares (in gray) inside of a circular dashed line (in blue). The detectors located at the opposite side of the $x$-ray source catch the signal transmitted by a square (signal inside the geometric shadow limited by the dashed lines). From the figure, in order to get sufficient spatial information for the reconstruction, more than one projection should be obtained to reproduce the square separations. Adapted from Buzug (Buzug, 2008).

Figure 2. Tomographic device built by Cormack in 1963. Adapted from Cormack (Cormack, 1980) 34

Figure 3. a) Prototype CT scanner by Hounsfield proposed to EMI Ltd., and b) an image of a preserved brain obtained with the equipment in 1968. Figures adapted from Cierniak (Cierniak, 2011).

Figure 4.a) First model of CT scanner, the EMI Mark I installed at the Atkinson Morley's Hospital in Wimbledon for clinical applications, and b) the brain transverse image of a patient with a suspected brain tumor taken in the same hospital. Figures adapted from Cierniak (Cierniak, 2011).....

Figure 5. Timeline evolution of CT technology, from 1971 to 2019. Adapted and updated from GE Healthcare (GE, 2019).

Figure 6. Block diagram of a CT system. Adapted from Hsieh (Hsieh, 2009).

Figure 7. a) CT scanner, model GE Discovery CT750 HD (image courtesy of GE Healthcare), Gantry and patient table, b) view of the gantry without its safety housing, which comprises the X-ray tube, collimator, the detector system, a high voltage generator, and controls. Adapted from Ulzheimer and Flohr (Ulzheimer and Flohr, 2009).

Figure 8. a) CT scanner representation with the associated three-dimensional Cartesian coordinate system (source: ImPACT 2001), b) and c) schematic diagram of a CT gantry with its major components: frontal view and lateral view, respectively. Figures adapted from Mahesh (Mahesh, 2009). 40

Figure 9. a) X-ray tube from 1978 with glass enclosure and rotating anode disk, b) schematic drawn of an X-ray tube and the process of X-ray production. The electrons are accelerated in the electric field between cathode and anode. $X$-ray radiation emerges from the deceleration of the fast electrons following their entry into anode material. Figures adapted from Buzug (Buzug, 2008)

Figure 10. X-ray spectra of a tungsten anode at acceleration voltages in the range of $\mathrm{Ua}=80-140 \mathrm{kV}$. The intensity versus photon energy (eV) plot shows the characteristic line spectrum as well as the continuous bremsstrahlung (courtesy of David, Philips Research Labs). The maximum energy is determined by the total energy, eUa, of the electron reaching the anode. Process illustrations: a) bremsstrahlung, b) characteristic emission, c) Auger process and d) direct electron-nucleus collision. Adapted from Buzug (Buzug, 2008). 45 Figure 11. a) Wehnelt cylinder used to forms and steers the electron beam onto a small focal point on the anode. Left: a dual-filament and Right: a modern mono-filament. b) Simulation of electron trajectories emitted from the filament and accelerated onto the anode. The potential at the Wehnelt cylinder controls the electron 
focus on the anode. Below: Shape and size of a large and small X-ray focus. Figures adapted from Buzug (Buzug, 2008) (courtesy of Philips Medical Systems).

Figure 12. a) Siemens's Straton X-ray tube and b) Its scheme, the anode directly attached to the tube envelope in cooling oil. The scheme also illustrates the alternating choose of two focal spots (courtesy of Siemens Medical Solution). Figures adapted from Buzug (Buzug, 2008).

Figure 13. $a-b)$ The sizes of the electronic focal spot, be, and the optic focal spot, b0, are determined by the orientation of the anode surface normal relative to the electron beam. Figures adapted from Buzug (Buzug, 2008).

Figure 14. Schematic representation of the target self-attenuation (heel effect). It is caused by attenuation of the X-ray beam by the anode. Figure adapted from Bushberg (Bushberg, 2002)

Figure 15. Beam geometry and profile characteristics, a) fan shaped X-ray beam, b) and c) dose profile from point and one-dimension focal spot. Adapted from Kalender (Kalender, 2011).

Figure 16. Beam hardening of an X-ray spectrum produced by a tungsten anode (anode angle $10^{\circ}$, acceleration voltage $\mathrm{Ua}=120 \mathrm{kV}$ ) due to flat shaped filters of $2.5 \mathrm{mmAl}$ and $0.2 \mathrm{mmCu}, 750 \mathrm{~mm}$ of air and also non-filtered spectrum is presented. The degree of absorption depends of the photon energy, in the figure, low energy photons are strongly absorbed. Figure adapted from Buzug (Buzug, 2008) (courtesy of B. David, Philips Research Labs.)

Figure 17. Impact of the bowtie filter attenuation on the $X$-ray fluence along fan angle. Bowtie filter reduces the incoming X-ray fluence (light-green profile) much higher at the periphery of the field than at the center due to its format, resulting in a more homogeneous photon fluence reaching the detection system (dark-green profile). Figure adapted from Bushberg et al. (Bushberg et al., 2011).

Figure 18. a) Sequential and b) helical CT scan mode. Figures adapted from Mahesh (Mahesh, 2009). c)

Schematic representation of the helical scan mode showing the nominal collimated beam width (width of active detector arrays) and the table feed per rotation of the gantry. Figure adapted from Bushberg et al. (Bushberg et al., 2011) 56

Figure 19. X-ray beam trajectories around the patient for different pitch values. $\mathrm{p}<1$ results in. .57 Figure 20. Schematic representation of the CTDI phantom used to measure the CTDI100. The holes at center and peripheral positions are used to put the $10 \mathrm{~cm}$ pencil ionization chamber to measure the center and peripheral CTDI100, respectively. Figures adapted from AAPM report 204 (AAPM, 2011). 59

Figure 21. CT dose descriptors based on the CTDI index. They are currently used for CT dosimetry evaluation. . 61 Figure 22. The total dose profile $\mathrm{f}(\mathrm{z})$ is the sum of a primary $\mathrm{fp}(\mathrm{z})$, and a scatter $\mathrm{fsz}$ component. Figure also shows a comparison with a simulated one.

Figure 23. Analytical primary profile $\mathrm{fp}(\mathrm{z})$, computed on the axis of rotation, AOR. The beam width a is $14 \%$ broader than the nominal one nT. Adapter from AAPM report 111 (AAPM, 2010)........................................64 Figure 24 Simplified geometrical model of a CT system. Figure adapted from (Dixon et al., 2005)....................65 Figure 25 Geometry for the generalized primary beam model. Figure adapted from (Dixon et al., 2005)..........67 Figure 26 Scheme to derive the primary dose profile at the central axis of rotation, AOR. 70 
Figure 27. Cumulated dose profiles DL(z), from the MC modelling of the Helical CT scan mode for a number $\mathrm{n}$ of $X$-ray source rotation. Along the a) central and b) peripheral axes of the head CTDI phantom.

Figure 28. Graphical representation of the methodology used to determine the effective diameter from the anterior-posterior, AP, and lateral dimensions of the patient cross section. The effective diameter corresponds to a circle having an area equal to that of the patient cross-section on a CT image. Figures adapted from AAPM report 204(AAPM, 2011).

Figure 29. Schematic representation of a CT device, highlighting the x-ray source model used to assess the dose profile and the $C T D I_{100}$ at center and peripheral positions. The orange line represents the position of the phase space plane, which serves to generate the phase space file.

Figure 30. Setup used to compute by MC simulation the primary dose profiles. Note that in this case, there is not CTDI phantom, only a cylinder of $1 \mathrm{~cm}$ diameter was located along the AOR to absorb the X-rays. .86

Figure 31. a) X-ray spectra generated by the TBC model used for the MC simulation, b) comparison between spectrum for $140 \mathrm{kV}$ and $6 \mathrm{~mm}$ Al with is corresponded measured one.

Figure 32. Geometrical characteristics and dimensions from Axial and Lateral view of the modelled bowtie filter. All numbers in the figure are in millimeters. Adapted from (thanks to João Terassi for AutoCAD design) ............88 Figure 33. Schematic representation to find the best position of the bowtie filter that 89 Figure 34. (a) cylindrical CTDI simulator used to compute the deposited energy at one center and four peripheral positions at $1 \mathrm{~cm}$ from the surface; (b) $3 D$ view showing the cylindrical bodies with $10 \mathrm{~cm}$ of length and $3 \mathrm{~cm}^{3}$ of volume.

Figure 35. Cumulated dose profiles, DL(z), of CT Helical examinations for a number of X-ray source rotations, from 1 to 10, along the a) central and b) peripheral axes of the head CTDI phantom. This data were obtained by Monte Carlo simulation with scan parameters, $n T=20 \mathrm{~mm}$, pitch=1.375, and $120 \mathrm{kV} X$-ray spectrum.

Figure 36. a) Body and head PMMA CTDI phantoms, pencil ion chamber, and radiation monitor. The CTDI phantoms have holes to introduce the pencil ion chamber. b) Schematic representation of the Experimental setup used to measure the CTDI100 in CTDI phantoms, and actual arrangement is shown at top-left of the figure which represent the alignment procedure using the red lasers of the CT device.

Figure 37. Experimental setup used to measure the CTDI100, air (free in air). In this case, the CTDI phantom is used as support.

Figure 38. OSL Ribbon dosimeters used to measure the Dose Profiles in CTDI phantoms. They have $20 \mathrm{~cm}$ length approximately.

Figure 39. a) and b) experimental setup used to measure the primary dose profiles, and c) single dose profiles.

They are measured using OSL ribbons.

Figure 40. Experimental setup for the measurements of the cumulative dose profiles in three CTDI body phantoms......

Figure 41. a) Reading system developed to register unidimensional dose profiles. It is based on the Pulsed Optically Stimulated Luminescence (POSL). b) Schematic representation of the reading system which uses LED to stimulate the OSL ribbon. The system is managed by an Arduino control board. Figures adapted from (Umisedo and Yoshimura, 2017). 
Figure 42. Calibration procedure of the simulated dose profiles, the corresponding CTDI100 values are used for the calibration. As the measurement of the CTDI100 is performed with a ion chamber of $10 \mathrm{~cm}$ length, the $10 \mathrm{~cm}$ central region of the dose profile was factored to its integral result in the CTDI100 value.

Figure 43. a) Primary profiles for four values, 40,30, 20 and $10 \mathrm{~mm}$, of the beam width onto the AOR, a, at $\varphi=7 \mathrm{o}$, the impact of the heel effect on the profile is indicated. b) Primary profiles computed for six values of the tilting angle of the focal spot, at a=20 $\mathrm{mm}$. The penumbra at both sides are indicated, also the influence of the heel effect when $\varphi=70$ at the left and right halves of the profile's upper part is indicated as well. All primary profiles were normalized to the unit at $z=0$, for better comparison, they were computed along the central axis of a CTDI phantom.

Figure 44. Scatter profiles for values of $138,30,20$, and $10 \mathrm{~mm}$ of the beam width, anto the AOR with a focal spot angle of $\varphi=70$. The central color region in each profile is inside the beam shadow and the black regions at both sides are out the beam shadow.

Figure 45. Total dose profile for values of 100, 80, 50, and $20 \mathrm{~mm}$ of the beam width, anto the AOR with a focal spot angle of $\varphi=70$. They are normalized to the unit at $z=0$.

Figure 46. User interface program implemented in MatLab R2012a environment (The MathWorks1 Inc., Natick, $M A, 2012$ ) showing the primary dose profile for a) central, b) peripheral phantom axis and c) the cumulative primary dose profile for central axis.

Figure 47. Primary profiles for head bowtie filter at nominal beam width of a) $\mathrm{nT}=20 \mathrm{~mm}$ and $\mathrm{b}$ ) $\mathrm{nT}=40 \mathrm{~mm}$. Measured using OSL ribbons for 80,100,120, and $140 \mathrm{kV}$ for both cases.

Figure 48. Primary profiles for body protocol (body bowtie filter) at nominal beam width of a) $\mathrm{nT}=40 \mathrm{~mm}$ and $\mathrm{b}$ ) $\mathrm{nT}=40 \mathrm{~mm}$. Measured using OSL ribbons.

Figure 49. Measured, theoretical, and simulated primary dose profiles a) for head bowtie filter at nominal beam width $\mathrm{nT}=20 \mathrm{~mm}$ and $\mathrm{b}$ ) body bowtie filter at nominal beam width $\mathrm{nT}=40 \mathrm{~mm}$. Experimental dose profiles were measured using OSL ribbons.

Figure 50. Dose profiles for a) head protocol at $\mathrm{nT}=20 \mathrm{~mm}$ and a) body protocol at $\mathrm{nT}=40 \mathrm{~mm}$. Measured using OSL ribbons at $120 \mathrm{kV}$. 114

Figure 51. Radiation background (BG) of dose profiles measurements.

Figure 52. Analytical (continuous) and simulated (dashed) primary profiles beam width, a, of 10, 20 and $27 \mathrm{~mm}$ onto the AOR.

Figure 53. (a) Comparison of analytical and simulated dose profile for 10, 20 and $27 \mathrm{~mm}$ of collimation projected onto the AOR. (b) Contribution of primary and scatter component on the dose profile for a cylindrical phantom with $32 \mathrm{~cm}$ diameter at $120 \mathrm{kV}$.

Figure 54. a) Central and b) Peripheral Dose Profile in Head CTDI phantom at 80kV and nT=20mm beam width. Comparison between simulated (black line) and experimental (red line) Dose Profiles.....

Figure 55. a) Central and b) Peripheral Dose Profile in Head CTDI phantom at $100 \mathrm{kV}$ and $\mathrm{nT}=20 \mathrm{~mm}$ beam width. Comparison between simulated (black line) and experimental (red line) Dose Profiles.

Figure 56. a) Central and b) Peripheral Dose Profile in Head CTDI phantom at $120 \mathrm{kV}$ and nT=20mm beam width. Comparison between simulated (black line) and experimental (red line) Dose Profiles. 
Figure 57. a) Central and b) Peripheral Dose Profile in Head CTDI phantom at $140 \mathrm{kV}$ and $\mathrm{nT}=20 \mathrm{~mm}$ beam width. Comparison between simulated (black line) and experimental (red line) Dose Profiles. 119 Figure 58. a) Central and b) Peripheral Dose Profile in Body CTDI phantom at 80kV and nT=40mm beam width. Comparison between simulated (black line) and experimental (red line) Dose Profiles. 120 Figure 59. a) Central and b) Peripheral Dose Profile in Body CTDI phantom at $100 \mathrm{kV}$ and nT=40mm beam width. Comparison between simulated (black line) and experimental (red line) Dose Profiles. 120 Figure 60. a) Central and b) Peripheral Dose Profile in Body CTDI phantom at $120 \mathrm{kV}$ and nT=40mm beam width. Comparison between simulated (black line) and experimental (red line) Dose Profiles. 120

Figure 61. a) Central and b) Peripheral Dose Profile in Body CTDI phantom at 140kV and nT=40mm beam width. Comparison between simulated (black line) and experimental (red line) Dose Profiles. 121 Figure 62. Sequential cumulative dose profiles along the central and peripheral axes of the head CTDI phantom. The imaging parameter is $\mathrm{nT}=10 \mathrm{~mm}$ in both cases and a) $\mathrm{I}=10 \mathrm{~mm}$ and $b$ ) $\mathrm{I}=15 \mathrm{~mm}$. Measured using OSL ribbons. Measurements for $120 \mathrm{kV}$. 122

Figure 63. Accumulative dose profiles in CTDI body phantom at central and peripheral positions a) pitch=1.375 and $\mathrm{nT}=40 \mathrm{~mm}$ and $\mathrm{b}$ ) pitch values: $0.531,0.969$, and 1.375 and $\mathrm{nT}=20 \mathrm{~mm}$ and $\mathrm{nT}=40 \mathrm{~mm}$. Measured using OSL ribbons. Measurements for $120 \mathrm{kV}$.

Figure 64. Accumulative dose profiles in CTDI body phantom for pitch values:0.531, 0.969, and 1.375, imaged volume length of $\mathrm{nT}=20 \mathrm{~mm}$ and $\mathrm{nT}=40 \mathrm{~mm}$, along the a) central and b) peripheral positions. Measured using OSL ribbons.

Figure 65. a) Central and b) Peripheral Dose profile for $120 \mathrm{kV}$ in term of air kerma (mGy/100 mAs) for pitch values: $0.531,0.969$ and 1.375 for head helical protocol. Both helical and axial dose profile correspond to a single rotation $(n=1)$ with beam width $20 \mathrm{~mm}$.

Figure 66. a) Peripheral and b) Central Dose profile for $120 \mathrm{kV}$ in term of air kerma (mGy/100 mAs) for pitch values: $0.516,0.984$ and 1.375 for body helical protocol. Both helical and axial dose profile correspond to a single rotation ( $n=1$ ) with beam width $40 \mathrm{~mm}$.

Figure 67. a) Central and b) Peripheral Dose profile for $120 \mathrm{kV}$ in term of air Kerma (mG/100 mAs) for pitch values: $0.531,0.969$ and 1.375 for head helical protocol. Both helical and axial dose profile correspond to a 10 rotations $(n=10)$ with beam width $20 \mathrm{~mm}$.

Figure 68. a) Central and b) Peripheral Dose profile for $120 \mathrm{kV}$ in term of air Kerma (mG/100 mAs) for pitch values: $0.516,0.984$ and 1.375 for body helical protocol. Both helical and axial dose profile correspond to a 10 rotations $(n=10)$ with beam width $40 \mathrm{~mm}$.

Figure 69. a) Dose profiles at central and peripheral axes for 0.531 pitch value and 1 and 10 rotations, b) Dose profiles at central and peripheral axes for 1.375 pitch value and 1 and 10 rotations. With $120 \mathrm{kV}$ and $20 \mathrm{~mm}$ beam width.

Figure 70. a) Dose profiles at central and peripheral axes for 0.516 pitch value and 1 and 10 rotations, b) Dose profiles at central and peripheral axes for 1.375 pitch value and 1 and 10 rotations. With $120 \mathrm{kV}$ and $40 \mathrm{~mm}$ beam width. 
Figure 71. a) Central and b) peripheral cumulated dose profile in head CTDI phantom for helical scan with

pitch $=1.375$.

Figure 72. a) Central and b) peripheral cumulated dose profile in body CTDI phantom for helical scan with pitch $=1.375$.

Figure 73. Simulated Dose Line Integral for head helical protocols of pitch values: 1.5, 1.375, 0.969, 0.75 and 0.531 at $120 \mathrm{kV}$ as a function of the scan length, L, a) along the central (DLIC) and peripheral (DLIp) axes and for b) Weighted-DLI, DLIw. Simulations were performed in an infinite cylindrical phantom of PMMA with $16 \mathrm{~cm}$ diameter and beam width of $20 \mathrm{~mm}$.

Figure 74. Simulated Dose Line Integral for body helical protocols of pitch values: $1.5,1.375,0.984,0.75$ and 0.516 at $120 \mathrm{kV}$ as a function of the irradiation length, L, a) along the central (DLIc) and peripheral (DLIp) axes and for b) Weighted-DLI, DLIw. Simulations were performed in an infinite cylindrical phantom of PMMA with $32 \mathrm{~cm}$ diameter and beam width of $40 \mathrm{~mm}$.

Figure 75. Pitch $x$ Equilibrium Dose product, pxDeq, for both head and Helical protocols at $\mathrm{nT}=20 \mathrm{~mm}$ and $\mathrm{nT}=40 \mathrm{~mm}$, respectively. Data is presented for the central and peripheral axis, and also the weighted Deq is presented, for both head and body helical protocols. The graph show clearly the dependence of the pxDeq with the tube voltage.

Figure 76. Cumulated dose profiles, DL(z), of CT Helical examinations for a number of X-ray source rotations, from 1 to 10, along the a) central and b) peripheral axes of the head CTDI phantom. This data were obtained by Monte Carlo simulation with scan parameters, $\mathrm{nT}=20 \mathrm{~mm}$, pitch=1.375, and $120 \mathrm{kV}$ X-ray spectrum. 134 Figure 77. Maximum dose at $\mathrm{z}=0$ of the cumulated dose profile, $\mathrm{DL}(0)$, from a helical scanning simulation at $80 \mathrm{kV}$ X-ray spectrum, a) DL(0) at the central and peripheral axes of the head CTDI phantom, and b) weighted$\mathrm{DL}(0)$ computed from $\mathrm{DL}(0)$ at central and peripheral axes.

Figure 78. Ratio Maximum Dose at $z=0$ to Equilibrium Dose, DL(0)/Deq at a) central and peripheral positions and $b$ ) weighted by the CTDIw coefficients for pitch values $0.531,0.969$ and 1.375 at $80 \mathrm{kV}$. 136 Figure 79. Ratio Maximum Dose at $z=0$ to Equilibrium Dose, DL(0)/Deq at a) central and peripheral positions and b) weighted by the CTDIw coefficients for pitch values $0.531,0.969$ and 1.375 at $100 \mathrm{kV}$. 136 Figure 80. Ratio Maximum Dose at $z=0$ to Equilibrium Dose, DL(0)/Deq at a) central and peripheral positions and b) weighted by the CTDIw coefficients for pitch values $0.531,0.969$ and 1.375 at $120 \mathrm{kV}$. 137 Figure 81. Ratio Maximum Dose at $z=0$ to Equilibrium Dose, DL(0)/Deq at a) central and peripheral positions and b) weighted by the CTDIw coefficients for pitch values $0.531,0.969$ and 1.375 at $140 \mathrm{kV}$. 137 Figure 82. Ratio Maximum Dose at $z=0$ to Equilibrium Dose, DL(0)/Deq at a) central and peripheral positions and b) weighted by the CTDIw coefficients for pitch values 0.531, 0.969 and 1.375 at $140 \mathrm{kV}$. 137 Figure 83. Ratio Maximum Dose at $z=0$ to Equilibrium Dose, DL(0)/Deq at a) central and peripheral positions and b) weighted by the CTDIw coefficients for pitch values $0.531,0.969$ and 1.375 at $140 \mathrm{kV}$. 138 Figure 84. Ratio Maximum Dose at $z=0$ to Equilibrium Dose, DL(0)/Deq at a) central and peripheral positions and b) weighted by the CTDIw coefficients for pitch values $0.531,0.969$ and 1.375 at $140 \mathrm{kV}$. 138 Figure 85. Ratio Maximum Dose at $z=0$ to Equilibrium Dose, DL(0)/Deq at a) central and peripheral positions and b) weighted by the CTDIw coefficients for pitch values $0.531,0.969$ and 1.375 at $140 \mathrm{kV}$. 
Figure 86. Dose Line Integral versus Dose Length Product for head CTDI phantom at $120 \mathrm{kV}$.

Figure 87. Simulated and measured dose profile comparison for Head CTDI phantom at $n T=20 \mathrm{~mm}$.

Figure 88. Simulated and measured dose profile comparison for Head CTDI phantom at $n T=50 \mathrm{~mm}$.

Figure 89. Simulated and measured dose profile comparison for Bead CTDI phantom at $n T=40 \mathrm{~mm}$.

Figure 90. Simulated and measured dose profile comparison for Bead CTDI phantom at nT=40mm.

Figure 91. a) Central and b) peripheral cumulated dose profile in head CTDI phantom for helical scan with pitch $=0.531$.

Figure 92. a) Central and b) peripheral cumulated dose profile in head CTDI phantom for helical scan with pitch $=0.969$.

Figure 93. a) Central and b) peripheral cumulated dose profile in head CTDI phantom for helical scan with pitch=1.375.

Figure 94. a) Central and b) peripheral cumulated dose profile in head CTDI phantom for helical scan with pitch $=0.531$.

Figure 95. a) Central and b) peripheral cumulated dose profile in head CTDI phantom for helical scan with pitch $=0.969$.

Figure 96. a) Central and b) peripheral cumulated dose profile in head CTDI phantom for helical scan with pitch $=1.375$.

Figure 97. a) Central and b) peripheral cumulated dose profile in head CTDI phantom for helical scan with pitch $=1.5$.

Figure 98. a) Central and b) peripheral cumulated dose profile in head CTDI phantom for helical scan with pitch $=0.75$.

Figure 99. a) Central and b) peripheral cumulated dose profile in head CTDI phantom for helical scan with pitch $=0.531$

Figure 100. a) Central and b) peripheral cumulated dose profile in head CTDI phantom for helical scan with pitch $=0.969$.

Figure 101. a) Central and b) peripheral cumulated dose profile in head CTDI phantom for helical scan with pitch $=1.375$......

Figure 102. a) Central and b) peripheral cumulated dose profile in head CTDI phantom for helical scan with pitch $=0.531$.

Figure 103. a) Central and b) peripheral cumulated dose profile in head CTDI phantom for helical scan with pitch $=0.969$.

Figure 104. a) Central and b) peripheral cumulated dose profile in head CTDI phantom for helical scan with pitch=1.375......

Figure 105. a) Central and b) peripheral cumulated dose profile in body CTDI phantom for helical scan with pitch $=0.516$.

Figure 106. a) Central and b) peripheral cumulated dose profile in body CTDI phantom for helical scan with pitch $=0.984$. 
Figure 107. a) Central and b) peripheral cumulated dose profile in body CTDI phantom for helical scan with pitch $=1.375$.

Figure 108. a) Central and b) peripheral cumulated dose profile in body CTDI phantom for helical scan with pitch $=0.516$.

Figure 109. a) Central and b) peripheral cumulated dose profile in body CTDI phantom for helical scan with pitch $=0.984$.

Figure 110. a) Central and b) peripheral cumulated dose profile in body CTDI phantom for helical scan with pitch=1.375.

Figure 111. a) Central and b) peripheral cumulated dose profile in body CTDI phantom for helical scan with pitch $=1.5$.

Figure 112. a) Central and b) peripheral cumulated dose profile in body CTDI phantom for helical scan with pitch $=0.75$.

Figure 113. a) Central and b) peripheral cumulated dose profile in body CTDI phantom for helical scan with pitch $=0.516$.

Figure 114. a) Central and b) peripheral cumulated dose profile in body CTDI phantom for helical scan with pitch $=0.984$.

Figure 115. a) Central and b) peripheral cumulated dose profile in body CTDI phantom for helical scan with pitch=1.375.

Figure 116. a) Central and b) peripheral cumulated dose profile in body CTDI phantom for helical scan with pitch $=0.516$.

Figure 117. a) Central and b) peripheral cumulated dose profile in body CTDI phantom for helical scan with pitch $=0.984$.

Figure 118. a) Central and b) peripheral cumulated dose profile in body CTDI phantom for helical scan with pitch=1.375.

Figure 119. Simulated Dose Line Integral for head helical protocols of pitch values: 1.375, 0.969 and 0.531 at 80 $k V$ as a function of the scan length, $L$, a) along the central (DLIC) and peripheral (DLIC) axes and for b) Weighted DLI, DLIW. Simulations were performed in an infinite cylindrical phantom of PMMA with $16 \mathrm{~cm}$ diameter and beam width of $20 \mathrm{~mm}$.

Figure 120. Simulated Dose Line Integral for head helical protocols of pitch values: 1.375, 0.969 and 0.531 at $100 \mathrm{kV}$ as a function of the scan length, $L, a)$ along the central (DLIC) and peripheral (DLIC) axes and for b) Weighted DLI, DLIw. Simulations were performed in an infinite cylindrical phantom of PMMA with $16 \mathrm{~cm}$ diameter and beam width of $20 \mathrm{~mm}$

Figure 121. Simulated Dose Line Integral for head helical protocols of pitch values: $1.5,1.375,0.969,0.75$ and 0.531 at $120 \mathrm{kV}$ as a function of the scan length, L, a) along the central (DLIC) and peripheral (DLIC) axes and for b) Weighted $D L I, D L / w$. Simulations were performed in an infinite cylindrical phantom of PMMA with $16 \mathrm{~cm}$ diameter and beam width of $20 \mathrm{~mm}$

Figure 122. Simulated Dose Line Integral for head helical protocols of pitch values: $1.375,0.969$ and 0.531 at $140 \mathrm{kV}$ as a function of the scan length, $L, a)$ along the central (DLIC) and peripheral (DLIC) axes and for b) 
Weighted DLI, DLIw. Simulations were performed in an infinite cylindrical phantom of PMMA with $16 \mathrm{~cm}$ diameter and beam width of $20 \mathrm{~mm}$

Figure 123. Simulated Dose Line Integral for body helical protocols of pitch values: $1.375,0.984$, and 0.516 at 80 $k V$ as a function of the irradiation length, L. Simulations were performed in an infinite cylindrical phantom of PMMA with $32 \mathrm{~cm}$ diameter and beam width of $40 \mathrm{~mm}$. 166

Figure 124. Simulated Dose Line Integral for body helical protocols of pitch values: 1.375, 0.984, and 0.516 at $100 \mathrm{kV}$ as a function of the irradiation length, L. Simulations were performed in an infinite cylindrical phantom of PMMA with $32 \mathrm{~cm}$ diameter and beam width of $40 \mathrm{~mm}$. 166

Figure 125 Simulated Dose Line Integral for body helical protocols of pitch values: 1.5, 1.375, 0.984, 0.75 and 0.516 at $120 \mathrm{kV}$ as a function of the irradiation length, L. Simulations were performed in an infinite cylindrical phantom of PMMA with $32 \mathrm{~cm}$ diameter and beam width of $40 \mathrm{~mm}$. 167

Figure 126. Simulated Dose Line Integral for body helical protocols of pitch values: $1.375,0.984$, and 0.516 at $140 \mathrm{kV}$ as a function of the irradiation length, L. Simulations were performed in an infinite cylindrical phantom of PMMA with $32 \mathrm{~cm}$ diameter and beam width of $40 \mathrm{~mm}$. 167

Figure 127. Equilibrium Length for $80 \mathrm{kV}$ head protocol 168

Figure 128. Equilibrium Length for $100 \mathrm{kV}$ head protocol 169

Figure 129. Equilibrium Length for $120 \mathrm{kV}$ head protocol 170

Figure 130. Equilibrium Length for $140 \mathrm{kV}$ head protocol 171

Figure 131. Equilibrium Length for $80 \mathrm{kV}$ body protocol 172

Figure 132. Equilibrium Length for $100 \mathrm{kV}$ body protocol 173

Figure 133. Equilibrium Length for $120 \mathrm{kV}$ body protocol 174

Figure 134. Equilibrium Length for $140 \mathrm{kV}$ body protocol 


\section{List of Tables}

Table 1. Approximations used to derive the analytical function for primary dose profile.

Table 2. Technical specifications of the GE Discovery 750HD.

Table 3. Physical parameters about the TBC spectra for 80, 100, 120, and 140kV. 87

Table 4. Main features of the servers available in the Group of Radiation Dosimetry and Medical Physics of the University oof São Paulo.

Table 5. Specifications (complies with IEC 61223-3-5 and IEC 606001-2-44 standards)

(https://www.sunnuclear.com/documents/datasheets/ctdi phantoms.pdf). 96

Table 6. Clinical body and head protocols chosen to be modelled by MC simulation. 98

Table 7. Measured CTDI100, air, CTDI100, $\mathrm{c}$ and CTDI100, $\mathrm{p}$ and computed CTDIw (according to Equation (14)) for head and body CTDI phantoms and for tube potentials of 80, 100, 120 and $140 \mathrm{kV}$. Nominal beam width, of $n T=10$, and $n T=20 \mathrm{~mm}$ for head phantom and $n T=10$, and $n T=40 \mathrm{~mm}$ for body phantom. For all measurements the systematic uncertainty (Type B) was $8.1 \%$, and the large focal spot was selected. Data for the GE Discovery CT750 HD scanner (General Electric Company, Boston, USA)......

Table 8. Ratios of CTDI100, c/CTDI100, p and CTDIw/CTDI100, air for head and body CTDI phantoms and for tube potentials of $80,100,120$ and $140 \mathrm{kV}$. For all cases, the beam width was set to $\mathrm{nT}=10 \mathrm{~mm}$. CTDosimetry (www.impactscan.org). $11.5 \%$ of uncertainty.

Table 9. Ratios of CTDI100, c/CTDI100, p and CTDIw/CTDI100, air for head and body CTDI phantoms and for tube potentials of $80,100,120$ and $140 \mathrm{kV}$. The beam width was fixed to $\mathrm{nT}=40 \mathrm{~mm}$ and $\mathrm{nT}=20 \mathrm{~mm}$ for body and head CTDI phantom respectively. CTDosimetry (www.impactscan.org). $11.5 \%$ of uncertainty.

Table 10. Percentage difference of CTDI100, c/CTDI100, $\mathrm{p}$ and CTDIw/CTDI100, air ratios between simulated, measured and values from CTDosimetry datasheet for tube potentials of 80, 100, 120 and $140 \mathrm{kV}$.

Table 11. a collimator aperture projected onto the AOR, a/nT is the over-beaming factor. Measured for this work on GE Discovery CT750 HD scanner, (Dixon and Ballard, 2007) on GE VCT scanner.

Table 12. Conversion coefficients to convert the dose profiles units from deposited dose per simulated history to Air-Kerma (mGy/100 mAs). Simulation over the head CTDI phantom with $n T=20 \mathrm{~mm}$.

Table 13. Conversion coefficients to convert the dose profiles units from deposited dose per simulated history to Air-Kerma (mGy/100 mAs). Simulation over the body CTDI phantom with $n T=40 \mathrm{~mm}$.

Table 14. Equilibrium Dose, Deq as a function of typical pitch values for Head helical protocols (a=20 $\mathrm{mm}$ ). Deq was obtained by fitting the DLI using a linear model where the intercept was fixed in 0 . The R-Square of the fitting was 1 for all cases.

Table 15. Equilibrium Dose, Deq as a function of typical pitch values for Body helical protocols ( $\mathrm{a}=20 \mathrm{~mm})$. Deq was obtained by fitting the DLI using a linear model where the intercept was fixed in 0 . The $R$-Square of the fitting was 1 for all cases. 
Table 16. Pitch value $x$ Equilibrium Dose, pxDeq as a function of typical pitch values for Head helical protocols (nT=20 mm).

Table 17. Pitch value $x$ Equilibrium Dose, pxDeq as a function of typical pitch values for Body helical protocols $(\mathrm{nT}=40 \mathrm{~mm})$

Table 18. Mean value of Pitch value $x$ Equilibrium Dose, pxDeq as a function of typical pitch values for Head (nT=20 mm) and Body $(\mathrm{nT}=40 \mathrm{~mm})$ helical protocols

Table 19. Equilibrium scan length, Leq, and scatter fraction, $\alpha$, for head helical protocol...............................140

Table 20. Equilibrium scan length, Leq, and scatter fraction, $\alpha$, for body helical protocol...............................140

Table 21. Scatter to primary ratio, SPR, for head helical protocol................................................................ 141

Table 22. Scatter to primary ratio, SPR, for body helical protocol............................................................. 141

Table 23. Scatter to primary ratio, SPR, for head and body helical protocols. ............................................. 142

Table 24. Equilibrium scanning length, Leq, for head and body helical protocols. ...................................... 142

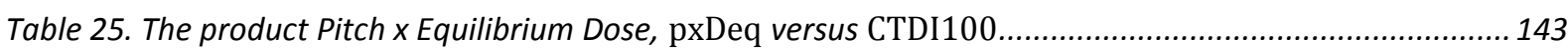

Table 26. CTDI100 values at central and peripheral axes, for tube voltages of 80, 100, 120, and $140 \mathrm{kV}$........148

Table 27. CTDI100 values at central and peripheral axes, for tube voltages of 80, 100, 120, and $140 \mathrm{kV} . \ldots . . .149$ 


\section{Acronyms}

AOR: Axis of Rotation

AI: Artificial Intelligence

CT: Computed Tomography

CTDI: Computed Tomography Dose Index

$\mathrm{CTDI}_{100, \text { air }}$ : Free in air Computed Tomography Dose Index

$\mathrm{CTDI}_{100, \mathrm{c}}$ : Central Computed Tomography Dose Index

$\mathrm{CTDI}_{100, \mathrm{p}}$ : Peripheral Computed Tomography Dose Index

CTDI $_{\mathrm{vol}}$ : Volumetric Computed Tomography Dose Index

$\mathrm{CTDI}_{\mathrm{w}}$ : Weighted Computed Tomography Dose Index

$\mathrm{D}_{\mathrm{eq}}$ : Equilibrium Dose

DLI: Dose Line Integral

FDA: Food and Drug Administration

IAEA: International Atomic Energy Agency

ICRP: International Commission on Radiological Protection

ICRU: International Commission on Radiation Units and Measurements

IEC: International Electrotechnical Commission

$\mathrm{L}_{\mathrm{eq}}$ : Equilibrium Scanning Length

MC: Monte Carlo

MDCT: Multi-Detector Computed Tomography

MSAD: Multi-Slice Averaged Dose

NCRP: National Council on Radiation Protection \& Measurements

PMMA: Polymethyl Methacrylate.

TLD: Thermoluminescent Dosimeter 


\section{Glossary}

$\tau=$ gantry rotation time $\left(360^{\circ}\right.$ rotation $) \approx 1 \mathrm{~s}$

$F=$ focal spot to axis of rotation distance $=541 \mathrm{~mm}$

$F_{\mathrm{c}}=$ focal spot to collimator distance along central ray $=162 \mathrm{~mm}$

$w=$ collimator aperture on central ray of x-ray tube.

$a=\left(F / F_{\mathrm{c}}\right) w \equiv M w=$ projected collimator aperture onto the AOR.

$M=F / F_{\mathrm{c}}=$ magnification factor.

$\alpha=$ anode target angle $=7^{\circ}$.

$s=$ focal spot length parameter in source plane $\left(s, x^{\prime}\right)$ parallel to anode surface

$z^{\prime}=s \sin \alpha=$ optical projection of $s$ perpendicular to central ray

$c_{0}^{\prime}=l \sin \alpha=$ focal spot length (optical projection perpendicular to central ray) $\approx 1 \mathrm{~mm}$

$c_{0}=(M-1) c_{0}^{\prime}=$ [penumbra width projected on central axis at $\mathrm{z}=0$ due to focal spot length $c_{0}^{\prime}$ (as if by slit on central ray)].

$g(s / l)=g\left(z^{\prime} / c_{0}^{\prime}\right)=$ focal spot relative emission intensity.

$z_{\alpha}=F \tan \alpha=$ scaling parameter (anode cutoff z-value on AOR) $=66.4 \mathrm{~mm}$.

$c^{\prime}(z)=\left(1-z / z_{\alpha}\right) c_{0}^{\prime}=$ apparent (optical) length of the tilted focal spot as viewed from a point $\mathrm{z}$ on the axis of rotation.

$c(z)=(M-1) c^{\prime}(z)=$ penumbra projected onto the AOR at $z$ by $c^{\prime}(z)$.

$\tilde{f}_{p}(z)=$ primary-beam axial dose profile with anode tilt but not including the heel effect

$f_{p}(z)=\rho(z) \tilde{f}_{p}(z)=$ primary beam axial dose profile with heel effect included.

$\rho(z)=$ heel effect modulation factor.

$\tilde{\rho}(z) \approx \rho(z)-\left\langle\mu_{p}\right\rangle R z^{2} / 2 F^{2}=$ [modified heel effect factor to include cone beam phantom attenuation for beams $>40 \mathrm{~mm}]$.

$\mu(E)=$ attenuation coefficient.

$\mu_{p}(E)=$ attenuation coefficient of the phantom

$\mu_{f}(E)=$ attenuation coefficient of the bowtie filter.

$k(E)=$ fluence to dose conversion factor.

$n T=$ nominal beam width.

$b=$ generalized CT table advance per rotation

$N=$ total number of gantry rotations in axial or helical scan series.

$v=$ table velocity for helical scans. 
$L=$ generalized definition of total scan length.

$R=$ radius of cylindrical phantom.

$\eta=$ scatter to primary ratio.

$p=b / n T=$ generalized pitch. 


\section{SUMARY}

\section{Table of Contents}

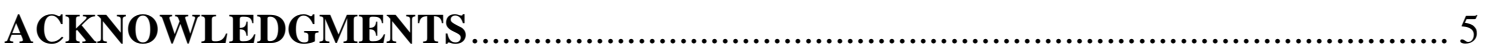

Abstract

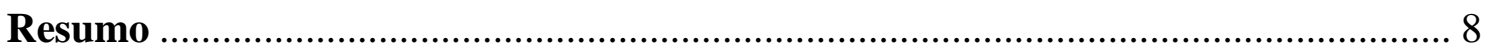

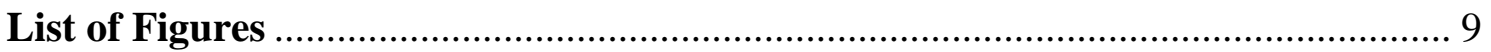

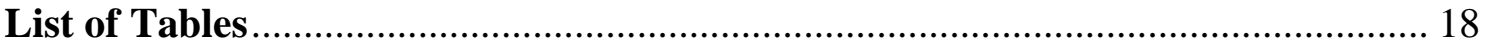

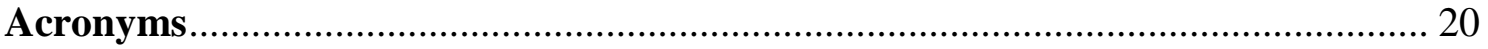

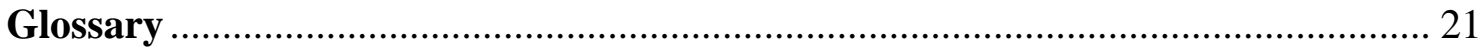

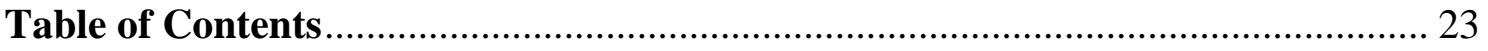

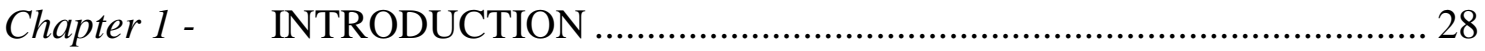

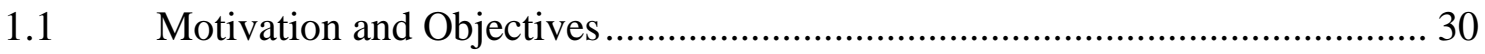

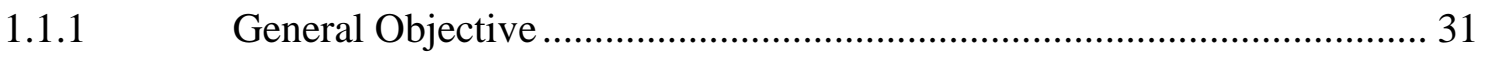

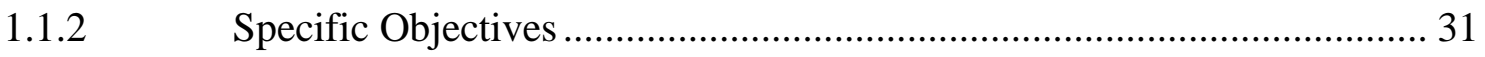

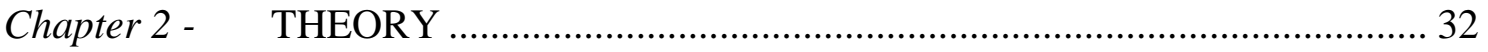

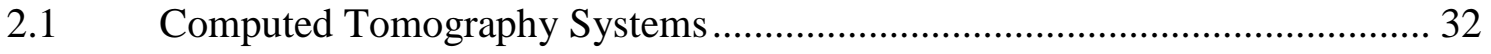

2.1.1 Brief history and evolution of CT scanners......................................... 32

2.1.2 Major components of a CT scanner..................................................... 37

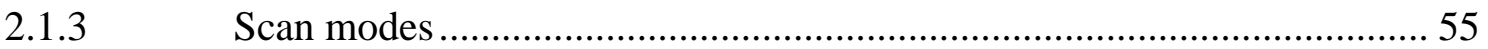

$2.2 \quad$ Current CTDI method for CT Dosimetry …............................................. 57

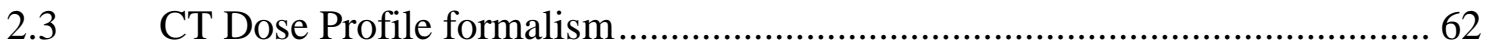

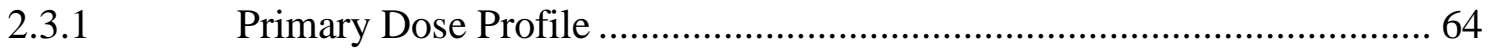

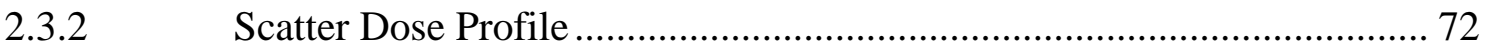

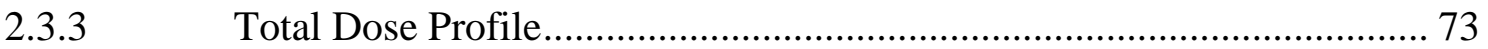

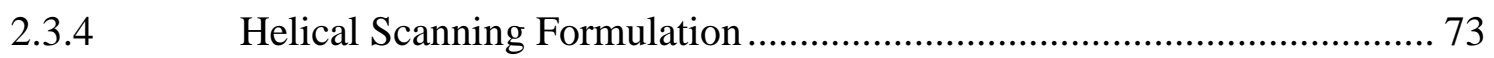

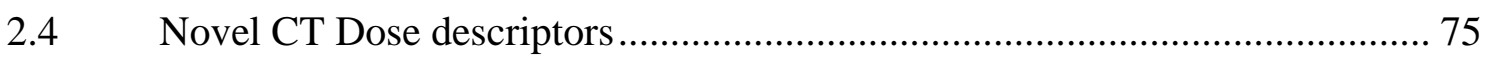

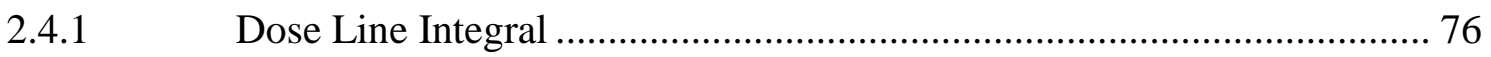

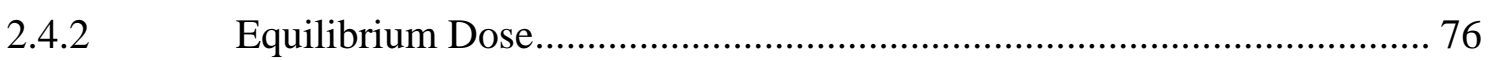




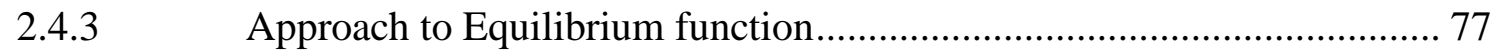

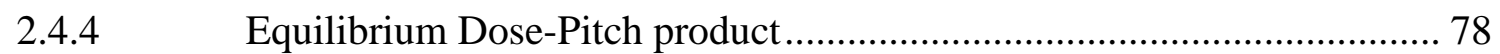

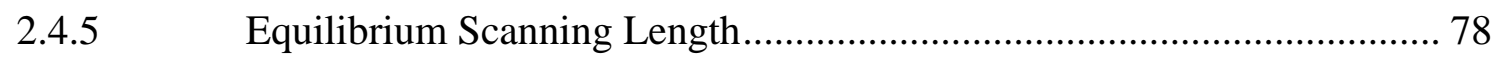

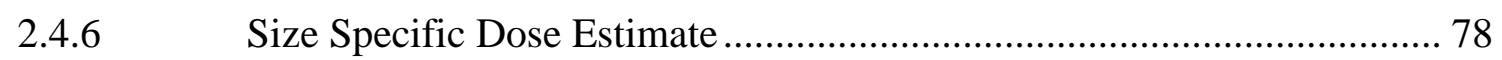

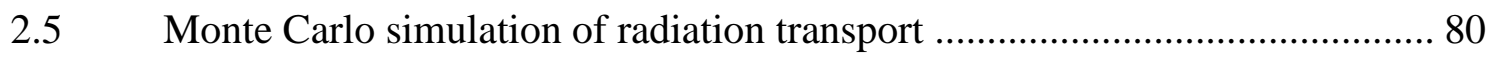

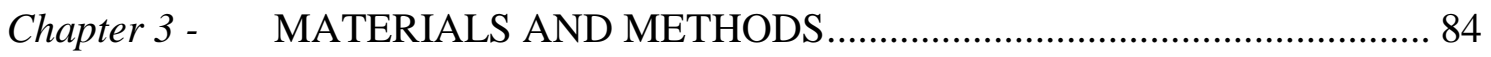

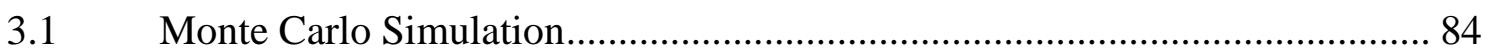

3.1.1 MC modelling of the CT X-ray emission ................................................. 84

3.1.2 Simulation of single and helical CT scanning ....................................... 90

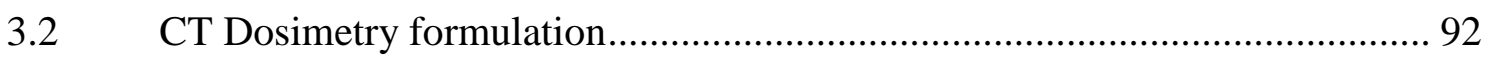

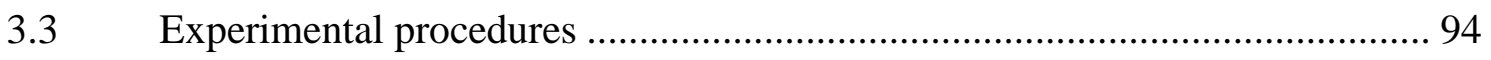

3.3.1 Computed Tomography Dose Index, CT DI100 _................................ 95

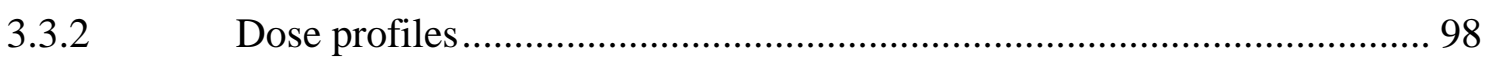

Chapter 4 - RESULTS AND DISCUSSIONS .................................................. 104

4.1 Validation of the X-ray source model........................................................ 104

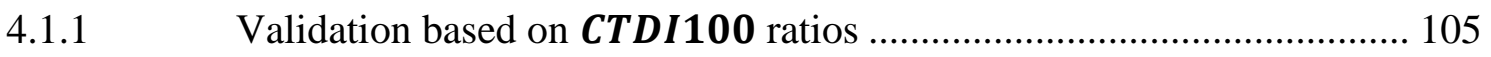

4.1.2 Validation based on dose profiles...................................................... 107

4.2 Cumulated Dose Profiles for Helical/Sequential Protocols .......................... 121

4.2.1 Cumulated Dose Profiles from experimental measurements ................. 122

4.2.2 Cumulated Dose Profiles from MC simulation ..................................... 124

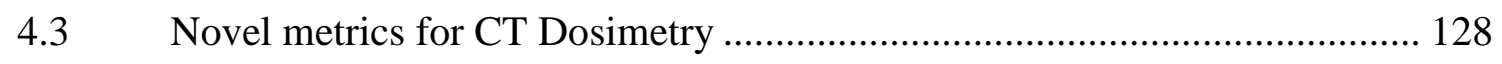

4.4 Evaluation of the efficiency of the CTDI100 method................................ 143

4.4.1 The product pitch x Equilibrium dose versus CT DI100 ..................... 143

4.4.2 Dose line integral versus dose length product ..................................... 143

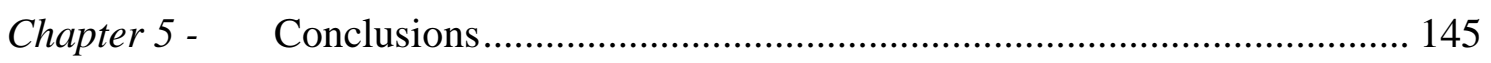

Appendix A: Experimental evaluation for the CTDI100 in GE Discovery CT750 HD148

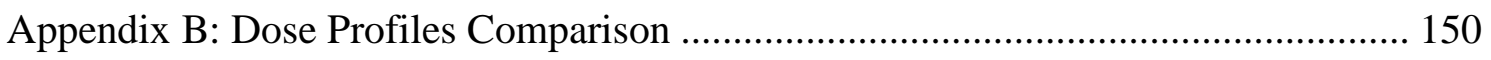

Appendix C: Cumulative Dose Profiles from MC simulation. ..................................... 154

Appendix D: Dose Line Integral versus Scan Length. .............................................. 164 
Appendix E: Rise to Equilibrium functions, assessing the Equilibrium Scanning Length, Leq. 168

List of publications, conferences papers, posters and training sessions 176

References. 178 


\section{Chapter 1 - INTRODUCTION}

The Computed Tomography scanner, CT scanner, is an X-ray machine which produces cross-sectional images which provides anatomical information of the human body (Marchal et al., 2005, Baert and Passariello, 2010, Schoepf and Meinel, 2016, Teasdale and Aitken, 2009). When the first clinical CT scanner was introduced in 1971 (Hounsfield, 1973, Ambrose, 1973), the biological effects of the dose produced by X-ray had already been described, mainly from researchers at hospitals (Crocker, 1897) or from reported cases in conventional radiography (BMJ, 1899). Therefore, several approaches to assess the radiation exposure $^{\mathrm{a}}$ and the radiation dose from a CT scanning were proposed. For instance, in the era of single CT slice, film dosimeters (Dixon and Ekstrand, 1978), thermoluminescent dosimeters (TLDs) (Shope et al., 1982) and ionization chambers (Jucius and Kambic, 1977, Suzuki and Suzuki, 1978, Kambic and Jucius, 1979, Moore et al., 1981) have been used to measure doses at selected locations in cylindrical phantoms and also dose profiles along the central axis perpendicular to the plane in which the CT X-ray tube rotates.

Aiming to have a practical approach to assess radiation exposure and radiation dose characteristics resulting from CT procedures consisting of a series of adjacent scans (or multiple scan procedure), the Computed Tomography Dose Index (CTDI) was introduced and formally defined in 1981 (Shope et al., 1981). CTDI is computed by total integration of the dose distribution resulting from a single CT scan, and it represented a practical method to characterize dose distribution from multiple scans. Nevertheless, a restricted version of the CTDI, the CTDI 100 was introduced by Leitz et al. in 1995 (Leitz et al., 1995) and rapidly adopted by the community. CTDI 100 integrates only $100 \mathrm{~mm}$ of the central region of the dose distribution resulting from a single CT scan. Therefore, it represents accurately dose characteristics resulting from a CT examination of specific $100 \mathrm{~mm}$ of scan length. Experimentally, $C T D I_{100}$ is measured using a pencil ion chamber of $100 \mathrm{~mm}$ length introduced in cylindrical phantoms of PMMA of $16 \mathrm{~cm}$ or $32 \mathrm{~cm}$ of diameter. This experimental procedure is adequately described in the AAPM report 96 (AAPM, 2008) and the IAEA/TRS 457 (IAEA, 2011).

The $C T D I_{100}$ is the current quantity used to track the radiation emission of a CT device, to be used in tests of acceptance and quality control, to compare CT protocols and

\footnotetext{
${ }^{\text {a }}$ Radiation exposure is a measure of the ionization of air due to ionizing radiation from photons; that is, gamma rays and X-rays. It is defined as the electric charge freed by such radiation in a specified volume of air divided by the mass of that air.
} 
even to be related with the patient absorbed dose. Nevertheless, the efficacy of the $C T D I_{100}$ to describe the radiation exposure resulting from a current CT examination has been challenged:

- Boone reported that the efficiencies of the $C T D I_{100}$ measured in the $32 \mathrm{~cm}$ diameter CTDI phantom were $63 \%$ and $88 \%$ for the center and peripheral holes, respectively, for beam width up to $40 \mathrm{~mm}$. This author also identified that efficiencies are reduced for beam width above $40 \mathrm{~mm}$ and down dramatically for beam width above $100 \mathrm{~mm}$ (Boone, 2007). This result causes concerns since the current CT devices incorporate the multiple-row detector technology that allows producing X-ray beams larger than $16 \mathrm{~cm}$. Moreover, the introduction of the cone beam technology allows the use of CT device in cardiac examinations, in which the CT table is maintained static. This CT procedure also challenges the use of the CTDI $_{100}$-paradigm $^{\mathrm{b}}$ as reported by Dixon and Boone (Dixon and Boone, 2014).

- The introduction of the Tube Current Modulation (TCM) as a dose reduction technique currently used in CT examinations, challenges the CTDI-paradigm defined as a shift invariant method for the single dose profile. In other words, the CTDI methodology considers that the cumulated dose profile from multiple slice CT examination can be computed from the single dose profile, using a simple mathematical operation. The TCM technique modules the radiation intensity emitted by the X-ray tube and therefore changes the dose profile from a single rotation along the scan length. This property is incongruent with the CTDI shiftinvariant paradigm (Dixon and Boone, 2013).

- Another important limitation of the $C T D I_{100}$ is that its measurement is limited to two phantom diameters. This fact causes problems to assess the patient absorbed dose since it does not take into account the variety of patient dimensions (AAPM, 2011), and its X-ray attenuation properties (AAPM, 2014a, AAPM-EFOMP, 2019). For instance, in Monte Carlo (MC) simulations of absorbed patient dose that take into account patient size, it has been shown that the dose increases much more slowly than does $C T D I_{100}$ or its derived metrics (Schmidt and Kalender, 2002, Turner et al., 2011).

\footnotetext{
${ }^{\mathrm{b}}$ Thomas Kuhn defined paradigms as "universally recognized scientific achievements that, for a time, provide model problems and solutions for a community of researchers (KUHN, T. S. \& HACKING, I. 2012. The Structure of Scientific Revolutions: 50th Anniversary Edition, University of Chicago Press.)
} 
From the aforementioned points, researchers are proposing to retire the $C T D I_{100}$ dose descriptor. In a point/counterpoint paper between David Brenner and Cynthia McCollough with Colin Orton as moderator (Brenner et al., 2006), Dr. Brenner emphasizes that the organ dose resulting from a CT examination obtained by direct measurement in anthropomorphic phantoms must be the dose descriptor in CT due to its direct association with radiationinduced cancer risk, where $C T D I_{100}$ have not direct association. On the other hand, Dr. McCollough defends the practical methodology based in the $C T D I_{100}$ because, despite its limitations, it represents a practical methodology for dose optimization that at the same time influences the organ dose. In 2010, the AAPM report 111 (AAPM, 2010) proposed a unified methodology for dose assessment that cover the axial/helical scan mode, the fan/cone beam geometry and for procedures with or without CT table movement. This methodology is based on the analytical formulation developed by Dixon (Dixon, 2019, Dixon, 2003, Dixon et al., 2005, Dixon and Ballard, 2007) that describes dose distribution in cylindrical phantoms, and also, proposes a new dosimetric method based on the measurement of the equilibrium dose with an ion chamber of $1 \mathrm{~cm}$ length inserted in a cylindrical phantom of sufficient length (Dixon, 2003, Dixon and Ballard, 2007). The dose descriptors proposed by the AAPM report 111 are the Dose Line Integral $(D L I)$, Equilibrium Dose $\left(D_{e q}\right)$, Equilibrium Dose-pitch product $\left(p D_{e q}\right)$, the approach to Equilibrium function $(H(L))$, and the Equilibrium Scanning Length $\left(L_{e q}\right)$.

\subsection{Motivation and Objectives}

This work aims to derive the dose descriptors proposed by the AAPM report 111 (AAPM, 2010) from the cumulated dose profiles produced in cylindrical phantoms of PMMA using Monte Carlo (MC) Method to simulate the couple electron-photon transport and to model a clinical helical CT scan mode. As mentioned above, the current $C T D I_{100}$ metric is a restricted version of the CTDI (as originally defined), which produces a reduction in the efficiency of CT dose estimation. Therefore, other dose descriptors derived from CTDI 100 may not be as accurate as desirable. This work incorporates in detail specific aspects of the radiation emission from an X-ray tube such as heel effect, focal spot angle, and X-ray spectrum, and therefore, our results include that detailed characteristics. Additionally, the analytical approach was evaluated to support its previous validations to confirm that the analytical approach is consistent and flexible for the different models of CT devices. 


\subsubsection{General Objective}

- Determination of the dose descriptors proposed by the AAPM report 111 to be applied in computed tomography using the Monte Carlo method.

\subsubsection{Specific Objectives}

- Study of the analytical formulation of the dose profiles in CTDI phantoms and the dose descriptors suggested by the AAPM report 111.

- Monte Carlo modelling of the radiation emission characteristics of a MDCT scanner and the helical scan acquisition mode.

- Validation and calibration of the Monte Carlo results with the air-kerma length product and measured dose profiles.

- Assessment of the deposited dose distribution inside the head and body CTDI phantoms by Monte Carlo simulation to determine the cumulated dose distribution along the central and peripheral phantom axes.

- Computation of the dose descriptors proposed by the AAPM report 111 from the simulated cumulative dose distribution corresponding to the helical CT scan mode. 


\section{Chapter 2 - THEORY}

This chapter conceptualizes the main topics developed in this work. It is structured as follows: Section 2.1 gives a brief history of the CT technology evolution, describes the CT major components, and defines the Helical/Axial CT acquisition protocols. Section 2.2 presents the current CT dosimetry methodology based on the CTDI 100 dose metric. Section 2.3 presents an overview about the analytical theory for CT dose profiles from single and multiple scans. Section 2.4 shows analytical equations for the new proposed CT dose descriptors. Finally, Section 2.5 comments about the PENELOPE/penEasy system that is a Monte Carlo based method to simulate the couple electron-photon transport in matter.

\subsection{Computed Tomography Systems}

\subsubsection{Brief history and evolution of CT scanners}

The entrance in the medical diagnostic scene of the CT technology depended of mathematical, computational and physical aspects. Mathematically, the CT technological development depended on the solution of the so-called inverse problem, which represents the image reconstruction of an object from its projections (or shadows). In general terms, the inverse problem had been studied by a number of prominent mathematicians along the $19^{\text {th }}$ century and the beginning of the $20^{\text {th }}$ century. A pioneer publication was performed by Abel in 1826 which showed how to calculate an axially symmetric emission function from its projection (Abel, 1826). In the beginning of the $20^{\text {th }}$ century, Radon presented a generalized method for image reconstruction introducing the Radon transform (Radon, 1917), which represented the basis for image reconstruction methods from projections. The graphic scheme in Figure 1 illustrates the basic idea of projection's formation in Xray technology applied to $\mathrm{CT}$, which helps us to understand the idea of the inverse problem. In Figure 1, three square elements (in gray) contained in a circular dashed line (in blue) are illuminated by an X-ray beam in two angular positions denoted by $\gamma_{1}$ and $\gamma_{2}$. The set of X-ray transmission signals form the functions $p_{\gamma_{1}}$ and $p_{\gamma_{2}}$, which represent the $\mathrm{X}$-ray signal that across each square region and arrive to a set of detectors located on the side opposite to the X-ray source. $p_{\gamma}(\xi)$ is the attenuation profile function of the beam versus the $\mathrm{X}$-ray detector coordinate array, $\xi$, under the projection angle, $\gamma$. Of course, to have the sufficient object spatial information to reconstruct an image, it is necessary to 
irradiate the object at a sufficient number of angular positions, $\gamma_{i}$, as is evidenced comparing the function $p_{\gamma_{2}}$ that gives information about the distance between the squares in Figure 1, in contrast to the function $p_{\gamma_{1}}$ where this information is missing. If $p_{\gamma_{i}}(\xi)$ is plotted over the all projection angles, then a bi-dimensional sinusoidal arrangement, or socalled sinogram, is obtained and represents the radon space of the object, essentially the set of raw data. In CT, the process of the object's image reconstruction from its sinogram is called the inverse problem (Buzug, 2008).

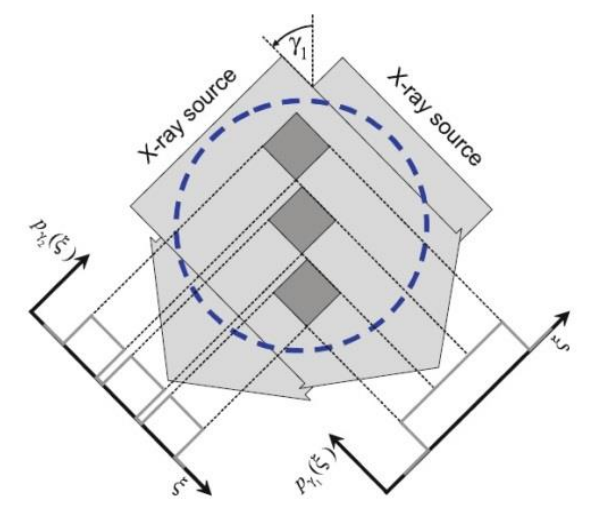

Figure 1. Schematic illustration to obtain attenuation profiles, $p_{\gamma_{1}}$ and $p_{\gamma_{2}}$, at two angular positions, $\gamma_{1}$ and $\gamma_{2}$, for a system composed by three squares (in gray) inside of a circular dashed line (in blue). The detectors located at the opposite side of the $x$-ray source catch the signal transmitted by a square (signal inside the geometric shadow limited by the dashed lines). From the figure, in order to get sufficient spatial information for the reconstruction, more than one projection should be obtained to reproduce the square separations. Adapted from Buzug (Buzug, 2008).

Computationally, image reconstruction requires huge computational resources, therefore, the development of computer fundamentals in the first part of the $20^{\text {th }}$ century boosted the advance of the CT technology in the second part of the $20^{\text {th }}$ century. Despite that others historical developments of a calculating machine (machine to do arithmetic operations) come from the abacus (familiar to the ancient Greeks and Romans), the Napier's bones (a system for the multiplication of numbers), the machine constructed by Pascal (to perform addition and subtraction) or the arithmometer, which was built independently by Baldwin and Odhner (Cierniak, 2011). It was the formalism developed by Turing in his paper: On Computable Numbers, with an Application to the Entscheidungsproblem (Turing, 1937) that could be regarded as the basis for the construction of current computers. It is important to note, that the increment of computing power is accompanied by the progress in the fields of electricity and electronics. Developments such us: the electronic valve (produced by Philips company in 1917), the transistor (developed by Americans Bardeen and Brattain in 1948) as well as the 
integrated circuit (developed by a group of researchers at Intel in 1969) permitted the miniaturization of computer components and the development of microcomputers to be used in CT technology (Cierniak, 2011).

Physically, in the beginning of the $20^{\text {th }}$ century, advance on understanding about the nature of the physical interaction of radiation with matter (radiation attenuation) had been achieved. Researchers concluded that the radiation attenuation follows an exponential behavior. In 1956 at the University of Harvard, Cormack began working on radiation attenuation in inhomogeneous materials tackling theoretically the problem of image reconstruction from X-ray projection (Cormack, 1963, Cormack and M., 1964, Cormack, 1973), after, confirming his results experimentally in 1963 using the apparatus shown in Figure 2. As additional information, Cormack started his studies in this subject after observing radiotherapy treatment plans in the Radiology Department, at the Groote Schuur Hospital, Cape Down. In that occasion, the isodose chart calculation for a treatment was computed in homogeneous materials, and A.M. Cormack thought, since humans have inhomogeneous bodies, how distorted the isodose chart would be by the inhomogeneities. This fact motivated Cormack on the computation of the attenuation coefficient distribution of tissues in a body and looking that these distributions had to be found by measurements made external to the body (Cormack, 1980).

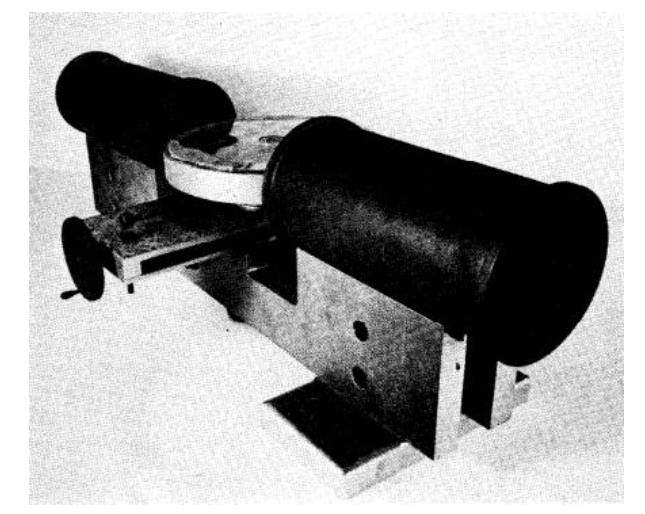

Figure 2. Tomographic device built by Cormack in 1963. Adapted from Cormack (Cormack, 1980)

In parallel to developments performed by Cormack, the Englishman Hounsfield, who was employed at the Central Research Laboratories of EMI Ltd., led the group that constructed the first CT scanner as a practical device developed in the late 1960s (Hounsfield, 1973, Ambrose, 1973). It is important to remark that both works were developed independently. Figure 3.a illustrates the CT scanner prototype developed by Hounsfield at the Central Research Laboratories of EMI Ltd., and Figure 3.b shows the 
CT image of a human brain prepared in formalin obtained with the equipment in 1968. In September 1971, the first CT system, the EMI Mark I illustrated in Figure 4.a, was installed for clinical applications at the Atkinson Morley's Hospital in Wimbledon. In October 1st 1971, the first tomographic examination of a patient brain was performed, as shown in Figure 4.b.

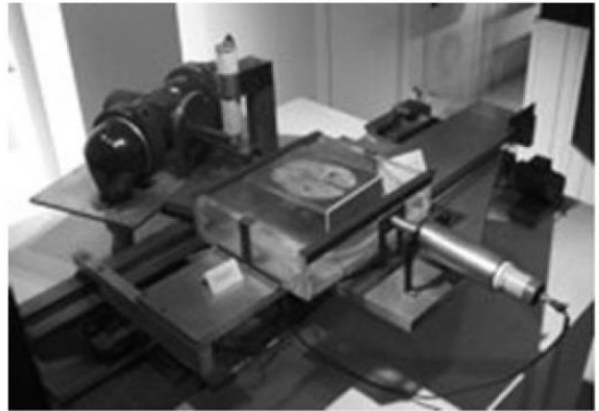

(a)

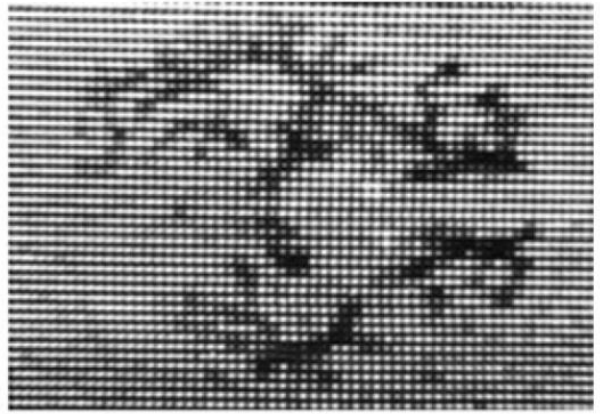

(b)

Figure 3. a) Prototype CT scanner by Hounsfield proposed to EMI Ltd., and b) an image of a preserved brain obtained with the equipment in 1968. Figures adapted from Cierniak (Cierniak, 2011).

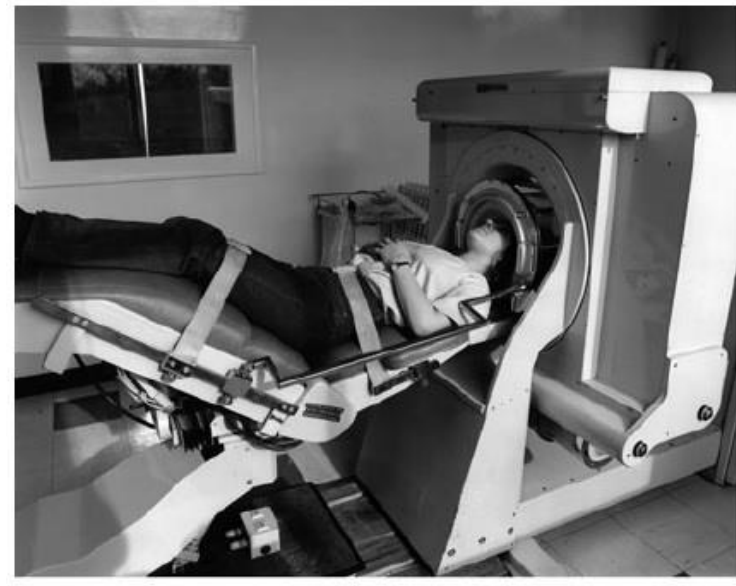

(a)

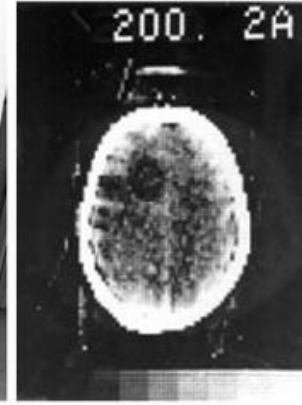

(b)

Figure 4.a) First model of CT scanner, the EMI Mark I installed at the Atkinson Morley's Hospital in Wimbledon for clinical applications, and b) the brain transverse image of a patient with a suspected brain tumor taken in the same hospital. Figures adapted from Cierniak (Cierniak, 2011).

From the EMI Mark I first clinical CT scanner installed in 1971 to the CT scanners used nowadays, remarkable advances have been incorporated which resulted in the improvement of image quality, reduction of acquisition time and reduction of X-ray exposure per reconstructed image (Pelc, 2014). Figure 5 shows a timeline of the CT technology evolution which highlights the main improvements on the X-ray tube, reduction in acquisition time, evolution of the detector system and the image reconstruction algorithm. For instance, in terms of the acquisition time, the first CT examination had taken about $4.5 \mathrm{~min}$ to rotate 
around the patient head in order to get transmission data for reconstructing one slice, while modern CT's acquisitions take up to less than $0.35 \mathrm{~s}$ for acquiring data to reconstruct until 320 slices. The time reduction for CT acquisitions represented the basic demand since the beginning of CT. The introduction of the slip ring technology in 1985 to allow the transfer of the necessary electrical energy for the X-ray tube by slip rings instead of cables permitted a continuous data acquisition and impulse the introduction of the Helical CT examination (Kalender, 2011). Special attention was taken as well for the detector system which its continuous developments allowed the use of broader fan beams to obtain several images per one gantry rotation, 320 slices per rotation under 2007 year. The number of detector rows was maintained from 2007 mainly because a broader beam imply large detector panel which increase the scatter, cone bean artifact, heel effect, and a potential tradeoff in image quality(Li, 2012). Recently photon counting detectors are being tested to be incorporated in clinical CT scanners (Lell and Kachelrieß, 2019). High power X-ray tubes are being introduced to be used in low $\mathrm{kV}$ scanning and also to switch the tube voltage on real time for better characteristics of CT exams. The software for image reconstruction is suffering an important evolution incorporating iterative algorithms (Renker et al., 2011, Schindera et al., 2013), for instance, the adaptive statistical iterative reconstruction algorithm (ASiR) introduced in 2007, automated image preprocessing, and machine learning algorithms have moved into focus today. For instance, the ACCIPIO $^{\circledR}$ ICH software which utilized deep learning technologies will be integrated on Philips' CT systems in 2019 (Maxq, 2019). All of the improvements allowed the use of CT in Cardiac (temporal resolution) (Achenbach and Kondo, 2012), dynamic (perfusion) CT, angiography and others examinations (Lell et al., 2015, Lell and Kachelrieß, 2019). Interesting technical, anthropomorphic, forensic, and archeological as well as paleontological applications of CT have been developed. These applications further strengthen the method as a generic diagnostic tool for non-destructive material testing and three-dimensional visualization beyond its medical use. Magnetic resonance imaging fails whenever the object to be examined is dehydrated. In these circumstances, CT is the three-dimensional imaging method of choice. Overall, new trends in medical imaging including new business models, artificial intelligence and the Internet of Things that create disruptive possibilities in health care can fund in this work (Alexander et al., 2019). 


\section{Timeline of CT}

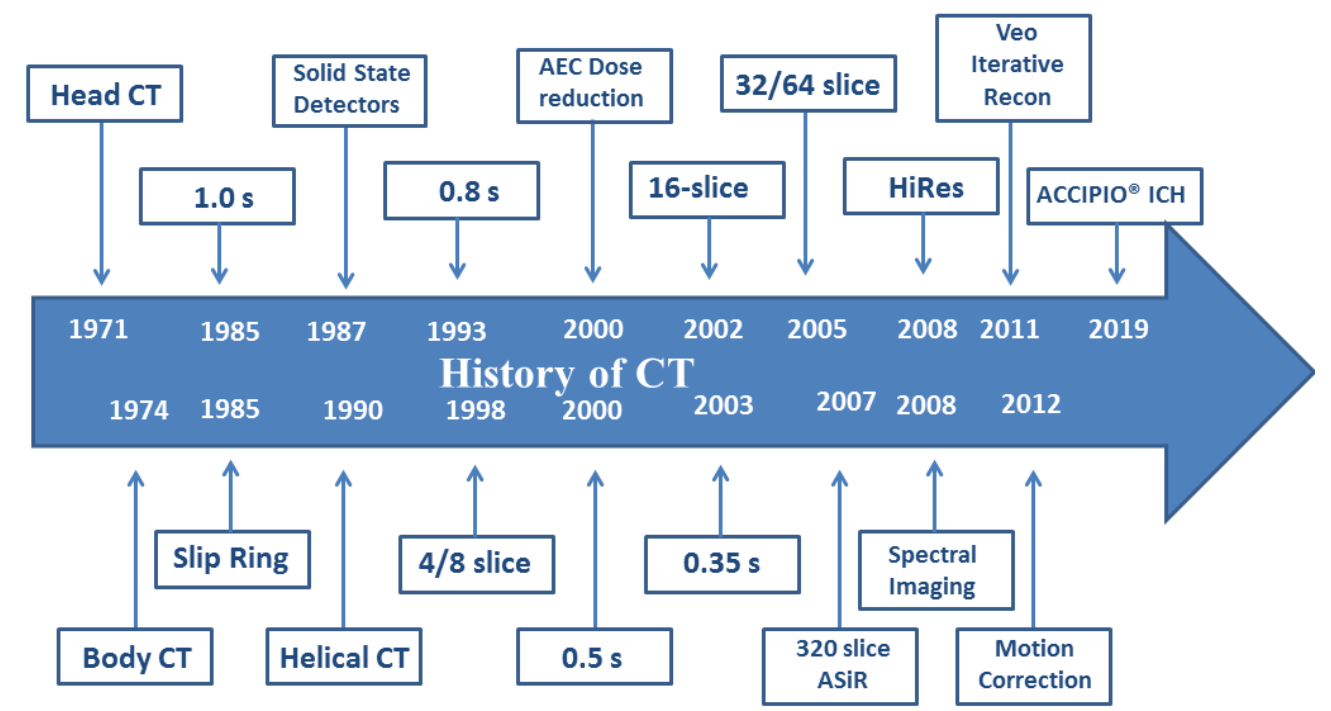

Figure 5. Timeline evolution of CT technology, from 1971 to 2019. Adapted and updated from GE Healthcare (GE, 2019).

\subsubsection{Major components of a CT scanner}

A Computed Tomography system integrates a number of elements adequately arranged in a radiology facility to guarantee high image quality, radiation safety for workers and patients and a confident quality assurance programme. Figure 6 illustrates a block diagram of a CT system where we can identify some of its elements, such as the operator console and displays, a computational system for image generation and a simple scheme of a CT scanner. Others, but not less important elements not shown in Figure 6 are the power generators, electronics and air conditioning. It is important to mention that the choice of the CT-system's computer software, for image reconstruction from the detector data (reconstruction algorithm), has considerable repercussion on image quality as well as on its diagnostic capacity, detailed information about reconstruction algorithms and a comparison between them can be found in (Zeng, 2010, Jensen et al., 2014, Schindera et al., 2013). Recently, Artificial Intelligence (AI) is being introduced in medical imaging for imagerecognition tasks and they are mainly implemented in Computed Tomography and Magnetic Resonance, more than $50 \%$ of the current articles about AI in medical imaging are in these areas, additional information can be found in (Pesapane et al., 2018, Hosny et al., 2018). 


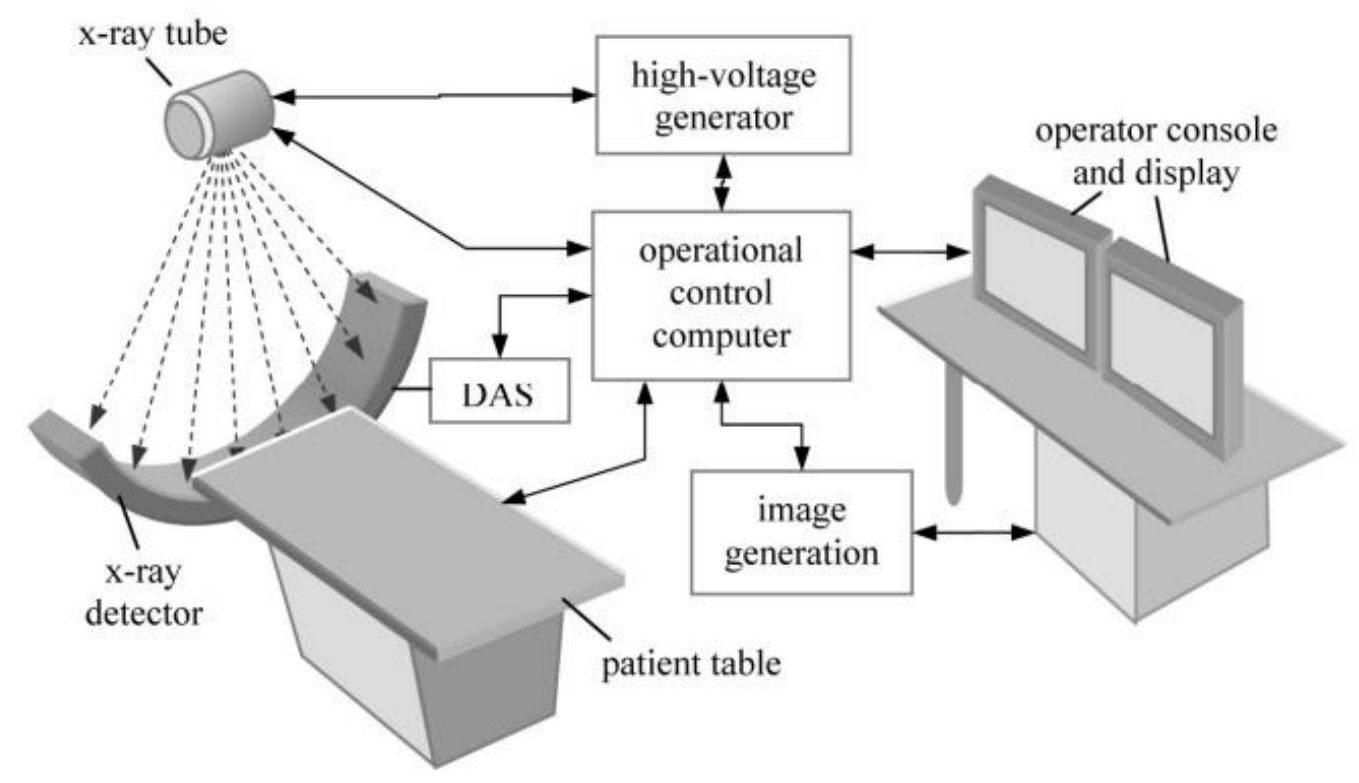

Figure 6. Block diagram of a CT system. Adapted from Hsieh (Hsieh, 2009).

CT scanners have similar dimensions for most manufactures, being their two major components the gantry and the patient table, as shown in Figure 7.a. In this section, we will describe the gantry's internal components and their geometrical arrangement. This description is important in this present study since their configuration impacts on the Monte Carlo modelling of the CT-scanner's radiation emission. Figure 7.b shows the gantry, which contains the main hardware of a CT scanner, without its safety housing where is possible to identify an X-ray tube system, the pre-patient collimator, the data measurement system, a high voltage generator and controls as well as their arrangement inside the gantry. Gantry's components weigh about $1,000-2,000 \mathrm{~kg}$, which rapidly rotate around the patient. They are inside a large housing to prevent patient mechanical injuries.
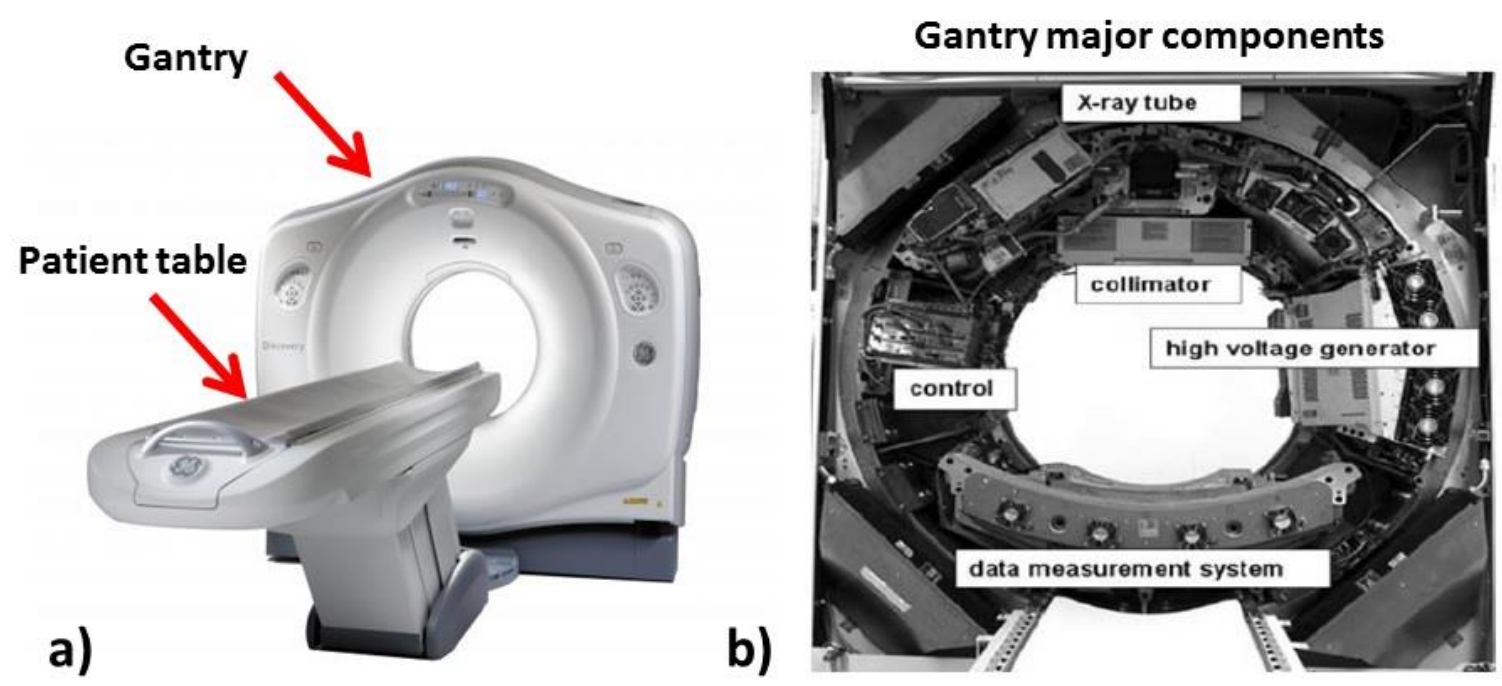
Figure 7. a) CT scanner, model GE Discovery CT750 HD (image courtesy of GE Healthcare), Gantry and patient table, b) view of the gantry without its safety housing, which comprises the X-ray tube, collimator, the detector system, a high voltage generator, and controls. Adapted from Ulzheimer and Flohr

(Ulzheimer and Flohr, 2009).

Before the gantry's major components description, three-dimensional Cartesian coordinate system must be defined for a formal description of the gantry rotation, CT component arrangement and MC modelling of the CT's radiation emission. Figure 8.a shows a CT-scanner's representation in which is possible to identify a three-dimensional Cartesian system with coordinates, $(x, y, z)$, and three conventional tomographic planes: $x-y$ (scan/transaxial plane), $x-z$ (coronal plane) and $y-z$ (sagittal plane). The origin point of the Cartesian coordinate system coincides with the CT isocenter, which is defined by the intersection between the gantry's AOR and the fan-beam central axis, as shown in Figure 8.c. Conventionally, the z-axis is aligned with the gantry's AOR pointing toward the patient table side (coming out from the page's plane in Figure 8.a) and the directions of the $x$ and $y$ axes are defined by the right-hand rule (plane $x-y$ is parallel to the page's plane). The gantry rotation occurs on a plane parallel to the $x-y$ plane (clockwise direction in Figure 8.a) and the couch movement is parallel to the $z$-axis. Additionally, Figure 8.a shows dashed red lines representing laser lights, which provide a reference to allow proper positioning and alignment of the patient or the CTDI phantoms.

Figure 8.b and Figure 8.c present the frontal (in-plane direction) and lateral (longitudinal direction) views, respectively, of a CT-gantry schematic diagram with its major components (Mahesh, 2009). Figure 8.b illustrates a schematic representation of the X-ray tube system (X-ray tube and filters), pre-patient collimator, patient and table positions, and the X-ray detectors. In this figure, the two continuous black lines represent a divergent X-ray fan beam shaped by the pre-patient collimator and attenuated by the patient and couch in its path toward the detectors. In MDCT, the fan beam aperture defines the scan field of view (SFOV) by circular areas commonly set around $50-55 \mathrm{~cm}$ in diameter. Also, the gantry opening has a circular shape up to $70 \mathrm{~cm}$ in diameter to allow the patient to pass through it during the scan (Mahesh, 2009). 

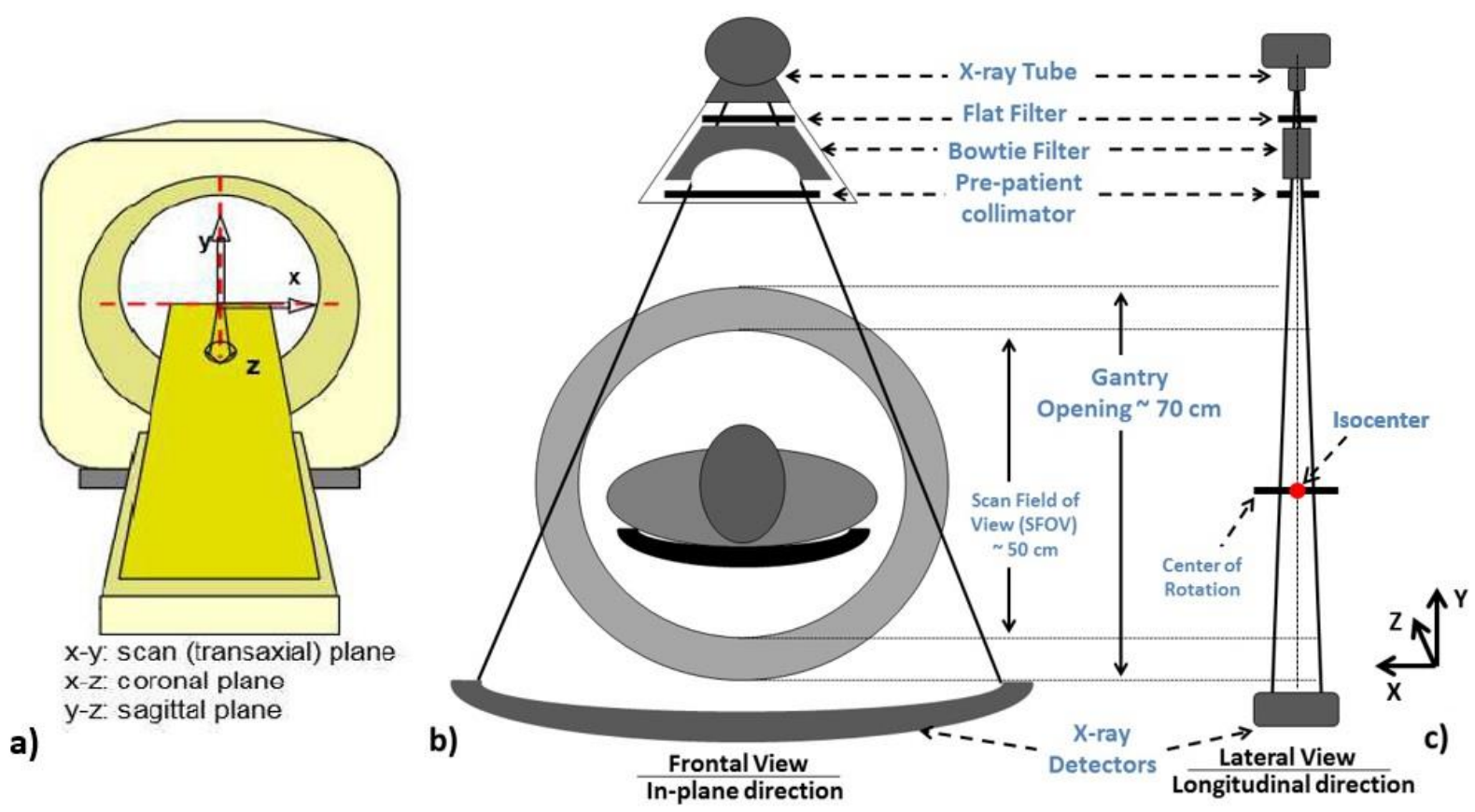

Figure 8. a) CT scanner representation with the associated three-dimensional Cartesian coordinate system (source: ImPACT 2001), b) and c) schematic diagram of a CT gantry with its major components: frontal view and lateral view, respectively. Figures adapted from Mahesh (Mahesh, 2009).

Next, the major CT components contained in the gantry will be described.

\section{- X-ray tube:}

$\mathrm{X}$-rays tubes are incorporated in most medical diagnostic devices. They represent the workhorse in medical diagnostic playing an important role in the success of most X-ray examinations. X-ray tubes have their major demand in CT scanners where they have contributed to spurring the rapid innovation of the CT technology (Mahesh, 2009). Figure 9 presents the basic X-ray tube's components which have been maintained along the time despite the CT-scanner broaden use and also despite its continue growing performance needed in a current CT examination. Figure 9.a shows an actual X-ray tube from 1978 constituent of a single-slice CT with scan time of 3 s per slice (Buzug, 2008) in which the rotating anode disk, the cathode and the tube housing (glass enclosure) can be identified. The X-ray tube housing assembly, comprise the vacuum electronics of an X-ray tube and means for radiation shielding, cooling, electrical insulation and mechanical interfacing (Behling and Grüner, 2018). Figure 9.b illustrates a schematic drawing of the X-ray production where a potential difference, $U_{a}$, is established between the anode disk and the cathode. Electrons produced in 
the filament by thermionic emission ${ }^{c}$ are accelerated by $U_{a}$ towards the anode disk. When the electrons impinge the anode surface, they interact with the atoms by various physical processes resulting into the X-ray emission which is represented geometrically in Figure 9.b by a cone beam. A vacuum condition is established inside the tube enclosure in order to avoid the contribution of electrons from the air to the electron stream, which would disrupt the Xray production process and damage the tube, and also to provide electrical insulation between the electrodes. The electron emission follows a thermionic process where the filament is directly heated to approximately $2,400 \mathrm{~K}$ to overcome the binding energy of the electrons from the metal of the filament ${ }^{\mathrm{d}}$ (Buzug, 2008).

The number of electrons delivered by the filament is essentially determined by the emission current density, $j_{e}$, which is a function of temperature and can be described by the Richardson-Dushman Equation (1).

$$
j_{e}=C_{R D} T^{2} e^{-\frac{\varphi}{k T}}
$$

Where $C_{R D}$ is the Richardson-Dushman ${ }^{e}$ constant, which is defined by Equation (2).

$$
C_{R D}=\frac{4 \pi m_{e} k^{2} e}{h^{3}}
$$

$k$ is the Boltzmann constant $\left(k=1.3810^{-23} J^{-1}\right)$ and $\varphi$ is the work function $(4.5 \mathrm{eV}$ for tungsten) which is the difference between the binding energy, $E_{v}$, and the Fermi energy edge. From the thermionic emission, an electron cloud is formed around the filament and these electrons are subsequently accelerated toward the anode. When the electrons reach the anode surface they will be stopped abruptly.

\footnotetext{
${ }^{c}$ Thermionic emission is the thermally induced flow of charge carriers from a surface or over a potential-energy barrier. This occurs because the thermal energy given to the carrier overcomes the work function of the material.

${ }^{\mathrm{d}}$ Filaments are usually made of thoriated tungsten with a melting point at $3,410^{\circ} \mathrm{C}$. Thoriated tungsten electrodes were introduced approximately fifty years ago as an alternative to the use of pure tungsten electrodes. The word "thoriated" means that each of the electrodes contains a small amount ( 1 or $2 \%$ by weight) of thorium dioxide (ThO2).

${ }^{\mathrm{e}}$ For ideal metals $C_{R D} \approx 120 \mathrm{Acm}^{-2} \mathrm{~K}^{-2}$. However, in practice $C_{R D}$ is material-dependent.
} 

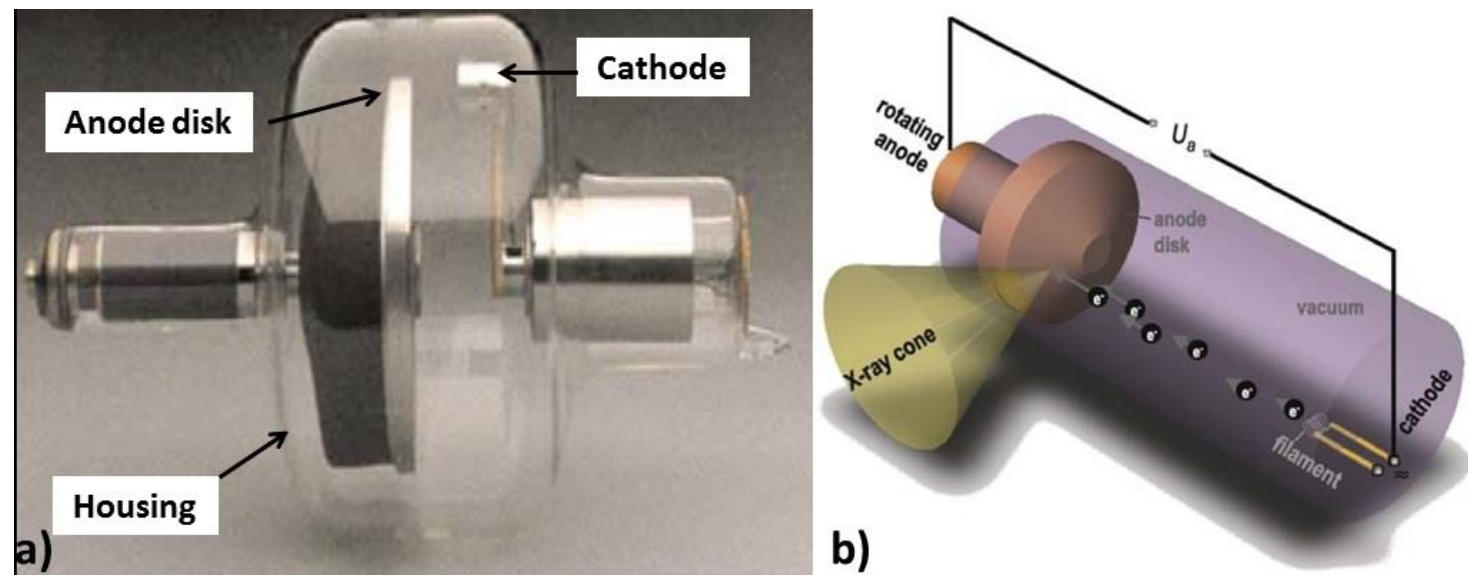

Figure 9. a) X-ray tube from 1978 with glass enclosure and rotating anode disk, b) schematic drawn of an $X$-ray tube and the process of $X$-ray production. The electrons are accelerated in the electric field between cathode and anode. $X$-ray radiation emerges from the deceleration of the fast electrons following their entry into anode material. Figures adapted from Buzug (Buzug, 2008)

As mentioned before, when the thermionic electrons reach the anode surface, they are decelerated by charged particle interactions via Coulomb force with atoms of the anode material. The accelerated electrons interact with the orbital electrons and the atomic nucleus resulting in several physical processes close to the anode surface which cause the electrontrajectory deflection and decrease of the electron kinetic energy producing electromagnetic wave emission, as supported by the classical electrodynamics (Jackson, 1975). The classical mechanism of charged particle interaction (Coulomb-force interactions) with matter can be classified according to the relation between the classic impact parameter, $b$, versus the atomic radius, $a$. According to Attix, a 'soft' collision occurs when, $b>>a$, a Hard 'knock-On' collision occurs when, $a \sim b$, and a Coulomb-force interaction with the external nuclear field occurs when $a>b$, detailed information about these kind of interactions can be found in (Attix, 1986). From the previously mentioned interactions, just the interaction with the nuclear field produces X-ray emission, where the emitted photon transports a fraction (up to $100 \%)$ of the electron kinetic energy ${ }^{\mathrm{f}}$. Such X-rays are referred to as bremsstrahlung, the German word for "braking radiation". The radiation emission due to the interaction with the nuclear field is significant for electrons and can be neglected for other more massive charger particles $^{\mathrm{g}}$ (Attix, 1986). Figure 10 depicts some kind of physical interactions between an accelerated electron with the field of orbital electrons and atomic nuclei, as well as, at the

\footnotetext{
${ }^{\mathrm{f}}$ Actually, around the 2-3\% of the cases in which the electron passes near the nucleus, an inelastic radiative interaction occurs in which an $\mathrm{X}$ ray photon is emitted.

${ }^{\mathrm{g}}$ Despite the differential atomic cross section for this inelastic collision is proportional to $\mathrm{Z}^{2}$, it also is proportional to the inverse square of the mass of the particle, for a given particle velocity. Thus bremsstrahlung generation by charged particles other than electrons is totally insignificant.
} 
bottom of the figure, four X-ray spectra corresponding to $80,100,120$ and $140 \mathrm{kV}$ potential difference are also presented. The X-ray spectra are expressed in units of photons per $\mathrm{mAs} . \mathrm{mm}^{2}$ at $0.75 \mathrm{~m}$ from the focal spot as a function of the photon energy in units of kiloelectronvolt, $\mathrm{keV}^{\mathrm{h}}$. Figure 10.a shows radiation emission processes in which an electron interacts with the atomic nuclear field, two electromagnetic-wave emissions (blue lines) are represented in this figure in which their wavelengths are related to the deflection degree of the electron trajectory. In Figure 10.a, the bigger deflection corresponds to the shortest radiation wavelength while a smaller deflection corresponds to the largest radiation wavelength. Despite that during the electron complete deceleration process several photons could be emitted, the emission of single photon with the whole electron kinetic energy is also possible. In this case, the maximum photon energy, $E_{\max }$, is equal to $e U_{a}$, which is the upper limit of the X-ray spectra presented in Figure 10. Therefore, it is possible to establish the following equations: $e U_{a}=h v_{\max }=E_{\max }$. From the equivalent expression for $E_{\max }$, it can be defined the minimum wavelength, $\lambda_{\min }$, as Equation (3).

$$
\lambda_{\min }=\frac{h c}{e U_{a}}=\frac{1.24 n m}{U_{a} / k V}
$$

In Equation (3), $h$ is the Planck's constant and $c$ is the speed of light ${ }^{\mathrm{i}}$. In the X-ray spectra shown in Figure 10, each spectrum interval is determined by the tube acceleration voltage and the intensity of each X-ray spectrum or number of X-ray quanta is controlled by the anode current. The slowing down of the electron kinetic energy throughout the electron path in the anode material is performed gradually by several interactions. Therefore, the energies of the emitted photons by this scheme form a continuous distribution of energies commonly called continuous radiation. Unfortunately, as mentioned before, the process of Xray generation by electron deflection is a rare one. The conversion efficiency from kinetic electron energy to bremsstrahlung energy can be described by Equation (4).

$$
\eta=K Z U_{a}
$$

Where $K$ is a material constant that was found by (Kramers, 1923) to theoretically ${ }^{\mathrm{j}}$ be $K=9 \cdot 2 \cdot 10^{-7} \mathrm{kV}^{-1}$ when the acceleration voltage, $U_{a}$, is given in $\mathrm{kV}$ (Buzug, 2008). For instance, from Equation (4), the conversion efficiency for a Tungsten anode $(\mathrm{Z}=74)$ and $U_{a}=140 \mathrm{kV}$ is roughly $1 \%$.

\footnotetext{
${ }^{\mathrm{h}}$ In physics, the electronvolt (symbol eV, also written electron-volt and electron volt) is a unit of energy equal to exactly $1.602176634 \times 10^{-19}$ joules (symbol J) in SI units.

i Planck's constant: $\mathrm{h}=6.626 .10^{-34} \mathrm{Js}$; speed of light in vacuum: $\mathrm{c}=2.998 .108 \mathrm{~m} / \mathrm{s}$.

j experiments are in good agreement with Kramers' result, but shown a slight dependence on Z.
} 
Figure 10.b shows a photon emission, denoted by $K_{\beta}$, produced by an electron transition from the M-shell to a vacancy in the K-shell. Vacancy was produced by a hard collision $(a \sim b)$ between a fast electron with a K-shell orbital electron, i.e., the atom is ionized by the loss of an inner electron, an electron of one of the higher shells fills the vacant position on the K-shell, as the inner shells represent states having a lower potential energy than outer shells, this process is accompanied by the emission of a photon. A photon produced by this process have characteristic-energy values which depend of the atomic element (atomic number), these photons are named characteristics X-rays and they appear in the spectrum as lines of high intensity, as shown in Figure 10. Overall, characteristic X-rays are produced when fast electrons collide with electrons of the atomic inner shells. The notation of this lines is agreed upon as follows: $K_{\alpha}, K_{\beta}, K_{\gamma}, \ldots$ denote the transition of an electron from the L-, M-, $\mathrm{N}-, \ldots$ shell to the K-shell and $L_{\alpha}, L_{\beta}, L_{\gamma}, \ldots$ denote the transition from the M-, N-, O-, .. shell to the L-shell, etc. The position of the characteristics K-line spectrum is given by Moseley's Law, shown in Equation (5).

$$
\lambda=\frac{h c}{E_{n}-E_{1}}=\frac{h c}{13.6 e V(Z-1)^{2}\left(1-1 / n^{2}\right)}
$$

Where $Z$ is the atomic number of the anode material and $n$ is the principal quantum number of the electron falling the K-shell. Despite, the characteristic-lines high intensity in the spectra shown in Figure 10, their contribution to the total spectrum is far less than the bremsstrahlung. Figure 10.c shows a non-radiative process in which the $K_{\beta}$ radiation is absorbed by an orbital electron of the same atom, as a consequence, the electron is released from the atom. This above described process is named the Auger process, for extension, the released electron receives the name of Auger electron. The probability of the Auger process is constant for all elements, however, this one competes with the characteristic radiation emission which probability is given by the ratio shown in Equation (6).

$$
P=\frac{Z^{4}}{Z^{4}+a}=\frac{1}{1+\frac{a}{Z^{4}}}<1
$$

Where $a$ is a positive constant ${ }^{\mathrm{k}}$. For elements with low atomic numbers, the Auger process dominates, whereas for heavy elements, characteristic emissions dominate. As mentioned before, the fast electron can lose the totality of its kinetic energy from a direct

\footnotetext{
${ }^{\mathrm{k}}$ For K-shell emission a $=1.1210^{6}$. OTENDAL, M. 2006. A Compact High-Brightness Liquid-Metal-Jet X-Ray Source Doctoral Thesis, Royal Institute of Technology..
} 
collision with the atomic nucleus, this process represents an ideal conversion of the entire kinetic energy of the electron to bremsstrahlung in one single deceleration process, as illustrated in Figure 10.d. Nevertheless, it can be concluded from the spectra shown in Figure 10 that direct collision is a very rare interaction process.

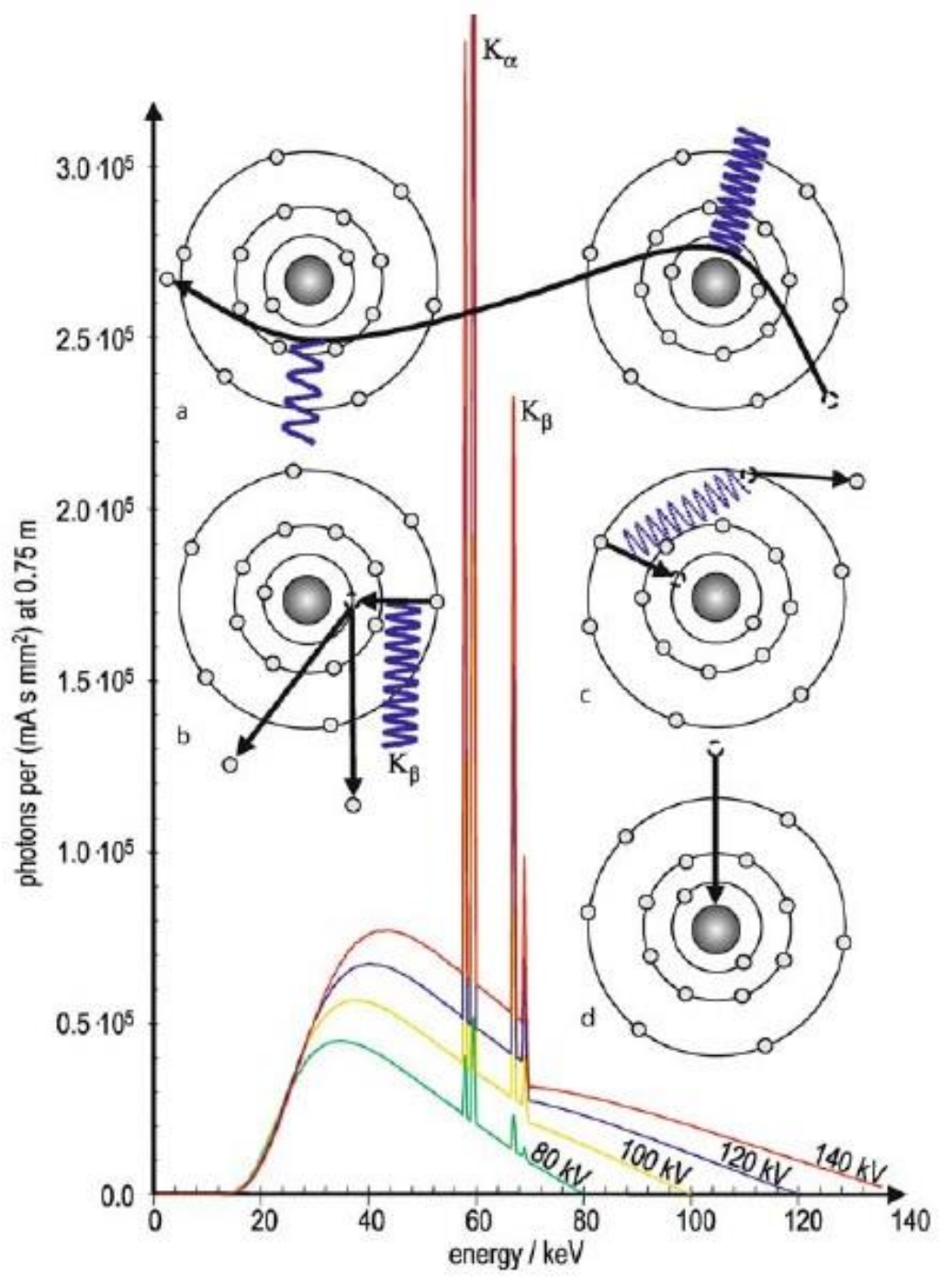

Figure 10. X-ray spectra of a tungsten anode at acceleration voltages in the range of $U_{a}=80-140 \mathrm{kV}$. The intensity versus photon energy $(\mathrm{eV})$ plot shows the characteristic line spectrum as well as the continuous bremsstrahlung (courtesy of David, Philips Research Labs). The maximum energy is determined by the total energy, $e U_{a}$, of the electron reaching the anode. Process illustrations: a) bremsstrahlung, b) characteristic emission, c) Auger process and d) direct electron-nucleus collision. Adapted from Buzug (Buzug, 2008).

The mean energy of the electron lost in matter can be quantitatively described by Equation (7).

$$
\left(\frac{d E}{d x}\right)=\left(\frac{d E}{d x}\right)_{\text {bremsstrahlung }}+\left(\frac{d E}{d x}\right)_{\text {ionization }}
$$


The first term in Equation (7) is given by quantum electrodynamics (QED), it is expressed in Equation (8).

$$
\left(\frac{d E}{d x}\right)_{\text {bremsstrahlung }}=-4 \alpha N_{A} \rho \frac{Z^{2}}{A}\left(\frac{e^{2}}{m_{e} c^{2}}\right)^{2} E \ln \left(\frac{183}{Z^{1 / 3}}\right)
$$

Where $\alpha$ is the fine structure constant ${ }^{1}, Z$ is the atomic number, $\rho$ is the density, $A$ is the atomic weight of the material, and $N_{A}$ is the Avogadro constant ${ }^{\mathrm{m}}$. The second term is presented in Equation (9).

$$
\left(\frac{d E}{d x}\right)_{\text {ionization }}=-4 \pi N_{A} \rho \frac{Z}{A}\left(\frac{e^{4}}{m_{e} c^{2}}\right) \frac{z^{2}}{\beta^{2}}\left[\frac{1}{2} \ln \left(\frac{2 m_{e} c^{2} \beta^{2} \gamma^{2} T_{\max }}{I}\right)-\beta^{2}-\frac{\delta}{2}\right]
$$

Where, the electron velocity is given in units of the speed of light, i.e., $\beta=\frac{v}{c}$, and, here, the charge of the electron $z$ is given in units of the elementary charge, therefore, $z=1$. $\gamma$ is the Lorentz factor, i.e., $\gamma=\left(1-\beta^{2}\right)^{-1 / 2}$. $T_{\max }$ is the maximum kinetic energy to be transferred in a single collision. $I$ is the mean ionization energy of the material and $\delta$ is a density correction of the ionization energy.

In order to focus the accelerated electron beam on the anode surface, an electron optics or Wehnelt optic (frequently named Wehnelt cylinder) system is employed. Figure 11.a presents the focusing device (Wehnelt cylinder) used to module the shape of the electron beam, basically, it is a cup-shaped electrode that forms the electric field near the filaments such that the electron current is directed to a small spot. Figure 11.b illustrates how the potential of the Wehnelt optics influences the electron trajectories, moreover, the potential can easily be controlled to produce a large or small X-ray focus.

\footnotetext{
${ }^{1}$ Fine structure constant: $\alpha=e^{2} /\left(4 \pi \varepsilon_{0} \hbar c\right) \approx 1 / 137.036$.

${ }^{\mathrm{m}}$ Avogadro constant: $N_{A}=6.02310^{23} \mathrm{~mol}^{-1}$.
} 


\section{Focusing device}

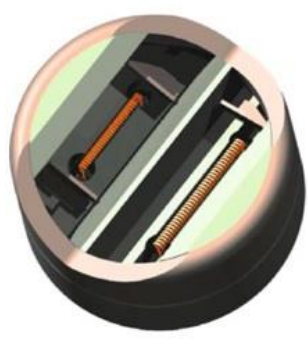

Dual-filament

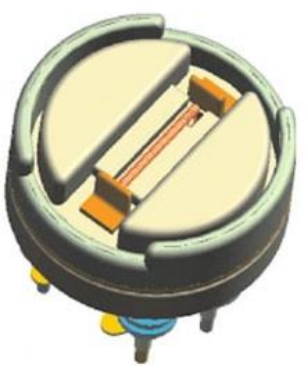

Mono-filament
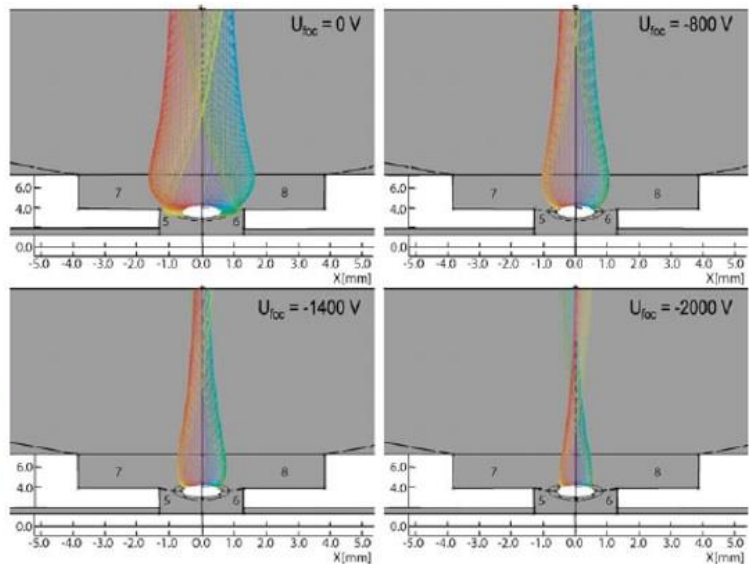

a)

b)
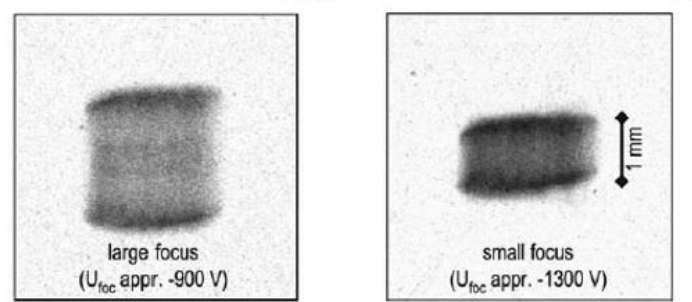

Figure 11. a) Wehnelt cylinder used to forms and steers the electron beam onto a small focal point on the anode. Left: a dual-filament and Right: a modern mono-filament. b) Simulation of electron trajectories emitted from the filament and accelerated onto the anode. The potential at the Wehnelt cylinder controls the electron focus on the anode. Below: Shape and size of a large and small X-ray focus. Figures adapted from Buzug (Buzug, 2008) (courtesy of Philips Medical Systems).

As mentioned before, the X-ray production is a low efficiency process, in which just a very small fraction of the incident energy is converted into X-ray radiation, for instance. In CT X-ray tubes roughly $1 \%$ of the incident energy is converted into X-rays, whereas $99 \%$ of the incident energy is convert into heat, this fact can be verified using Equation (4). Therefore, it can be inferred that X-ray tubes have serious heating problems more so for those that are used in high-performance CT devices where high-intensity X-ray beams are needed. In $\mathrm{CT}$ examinations, the tube current, $I$, and the exposure time, $t$, or, more precisely, the product of current in milliamperes $(\mathrm{mA})$ and exposure time in seconds (s) are two important parameters that the radiologists need to choose adequately. The heat capacity of X-ray tubes measured in Head Units (HU) can be computed from Equation (10). Common values of tube head capacity used in multi-slice CT systems are 5-8 106 HU (Buzug, 2008).

$$
H U=U . I . t
$$

Since that, in a CT examination, long time exposure (especially for spiral scanning on large anatomical regions) and high intensity beams (for high values of table speed) are required, X-ray tubes with a good head capacity are needed in order to avoid melting the anode or break the tube housing. Several strategies were developed to deal with the anode 
disk heating problem, one of the them was the introduction of the rotation anode disk, invented in 1919 and introduced in 1929 (Otendal, 2006). Previously in 1896, the year after Roentgen's discovery of x-rays, the American physicist R.W. Wood suggested that the ebeam power load capacity of an anode would increase if it could be rotated during the exposure (Wood, 1896). However, the complexity of such an X-ray tube delayed the introduction of rotating-anode sources on the market until 1929 when Philips presented their Rotalix tube 1929. In which rather than concentrating the energy of the electron beam at one reduced region, it is spread out over a line, called the focal line. Rotating anodes are disks mounted on a rotor assembly supported by bearings in the X-ray tube insert. The disk is made of graphite, with tungsten or tungsten-rhenium alloy deposited in one face via chemical steam process. Tungsten provides great bremsstrahlung production, graphite increases the heat capacity in the disk by a factor of 10 , in comparison with a disk made of pure tungsten, and rhenium provides added resistance to surface damage. In design of X-ray tube anode, materials with a large, $Z$, high thermal conductivity, $\lambda_{t}$, and a maximum melting point temperature, $\vartheta_{\max }$, must be chosen. The expression $Z \vartheta_{\max } x\left(\lambda_{t} \rho c\right)^{1 / 2}$ must be optimized, where $\rho$ is the mass density and $c$ is the heat capacity (Buzug, 2008). Tungsten fulfills these requirements (Morneburg and Aktiengesellschaft, 1995). Other developments change the strategy for tube cooling, an example is the Straton ${ }^{\circledR}$ X-ray tube (Siemens Medical Solutions, Erlangen, Germany), shown in Figure 12.a where the whole tube housing rotates completely embedded in cooling oil, as illustrated in Figure 12.b, achieving cooling rates values of about 4.8 MHU/min. This tube utilizes convective cooling rather than the radiation process. The Straton ${ }^{\circledR}$ X-ray tube is the first tube for the class of rotating enveloped tubes introduced in 2003 to be used in CT (Schardt et al., 2004). Its high cooling rate allows to eliminate the need for a large heat storage capacity at the anode and therefore allowing a smaller tube size. Thanks to its low weight, this kind of X-ray device has been integrated into the current stateof-the-art dual source generation of CT scanners (Buzug, 2008). A latest development in the X-ray tube technology is the Philips iMRC® (Philips Healthcare, Hamburg, Germany) tube, which implement double quadrupole and dipole for magnetic focusing, incorporated in the Brilliance $\mathrm{iCT} \circledast$ and spectra detection IQon ${ }^{\circledR}$ scanner families (Philips Healthcare, Andover, MA). For more information about this recent X-ray tubes developments, see, (Shefer et al., 2013, Behling and Grüner, 2018, Behling, 2015). As described above, the current clinical CT 
devices produce X-ray radiation based on the bremsstrahlung process, for alternative ways to produce X-ray radiation for imaging purposes see, (Behling and Grüner, 2018).

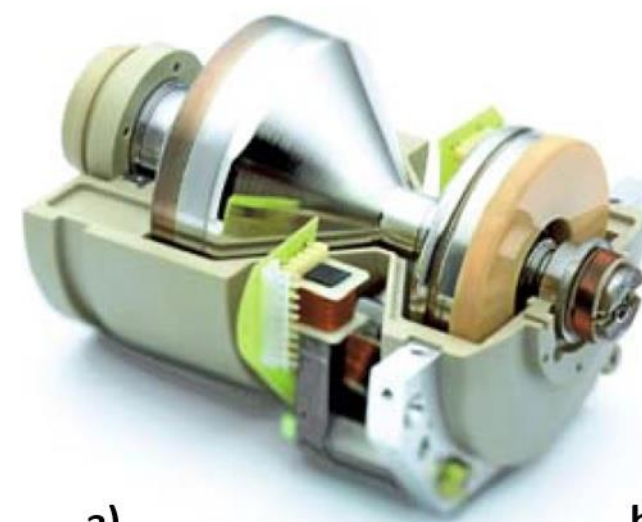

a)

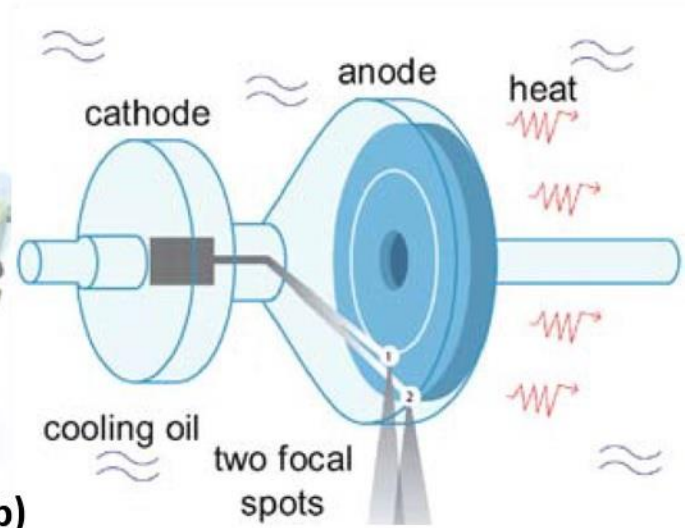

b) spots

Figure 12. a) Siemens's Straton X-ray tube and b) Its scheme, the anode directly attached to the tube envelope in cooling oil. The scheme also illustrates the alternating choose of two focal spots (courtesy of Siemens Medical Solution). Figures adapted from Buzug (Buzug, 2008).

As illustrated in Figure 11.b, X-rays are produced in a non-point focus, this fact causes important influence in the dose-profile's shape (especially in the primary profile) and in the quality of the resulting image. Overall, image quality is degraded when a large-focus is used. A large-diameter focus produces a penumbra formation in the image which causes its blurring and loss of its sharpness. A detailed explanation of the image quality dependence with the focus size is not the scope of this work, more information about this issue with a good mathematical formalism can be found in Chap.9 of Buzug (Buzug, 2008). The focus area or also called focal spot is the anode-surface area where the electron-beam front strikes the anode (the X-ray focus diameter for diagnostic X-ray tubes is typically found to be between $0.3 \mathrm{~mm}$ and $2 \mathrm{~mm}$ ), the diameter of the X-ray focus depends on the diagnostic application, see for examples, (Huda, 2010). This area defines the electronic focal spot length, $b_{e}$, which is the focal spot dimension on the anode surface parallel to the page plane and it depends on the angle, $\varphi$, between the beam direction and a ray perpendicular to the anode surface, as shown in Figure 13. Moreover, the projection of the focal spot length on an axis that coincide with the electron beam direction define the optical focal spot, $b_{0}$, which can be understood as the focal-spot's length seen from a point located on the detector system, mathematically, $b_{0}$, is defined by $b_{0}=b_{e} \cdot \sin \varphi$ and is also illustrated in Figure 13. When the angle, $\varphi$, becomes larger, the electronic focal spot length increases as well. This fact increases the tube power limit, because it allows the heat to be deposited across a relatively large spot delivering the 
electron kinetic energy in a bigger area. At the same time, the optical focal spot length becomes larger too, which causes artifact in the final image (loss of image sharpness).
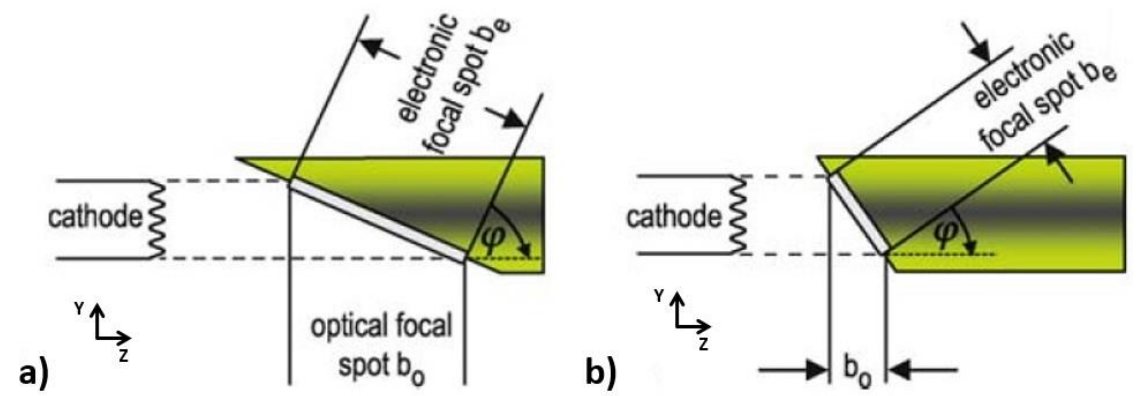

Figure 13. a-b) The sizes of the electronic focal spot, $b_{e}$, and the optic focal spot, $b_{0}$, are determined by the orientation of the anode surface normal relative to the electron beam. Figures adapted from Buzug (Buzug, 2008).

\section{- Heel effect}

Figure 14 illustrates the anode self-attenuation effect on the emitted X-ray beam intensity. As can be identified in the polar coordinate system, the X-ray beam portion at the anode side (dark beam region) is more attenuated than the portion at the cathode side (clear beam region). The X-ray production is performed along the electron route during its path inside the anode, actually, the penetration depth of electrons in the anode depend on the kinetic energy and the anode material and can be found up to $30 \mathrm{um}$, for diagnostic voltages. Basically, the $\mathrm{X}$-ray production follows an isotropic emission, nevertheless, depending on the path direction followed to exit the anode, the X-rays travels different distances which imply asymmetric attenuation, moving away from the ideal uniform beam, which is the paradigm for image reconstruction in $\mathrm{CT}^{\mathrm{n}}$. The mentioned effect is called the Heel Effect, which has important consequences for broader X-ray beams. In Figure 14, the anode surface is tilted $15^{\circ}$ with respect to a central ray (y-axis direction), the beam is limited by a collimator to cover $30^{\circ}$ in the polar coordinate system. The dashed black line represents a percentage of the exposure output at $15^{\circ}$ of emission angle (left side in the figure), which is reduced up to roughly $25 \%$ due to the heel effect, as can be seen in Figure 14. Consequence of the Heel Effect on the primary and total dose profile will be described in Section 4.1.2.

\footnotetext{
${ }^{\mathrm{n}}$ the paradigm for image reconstruction in CT is that the object to be scanned must be illuminated homogeneously.
} 


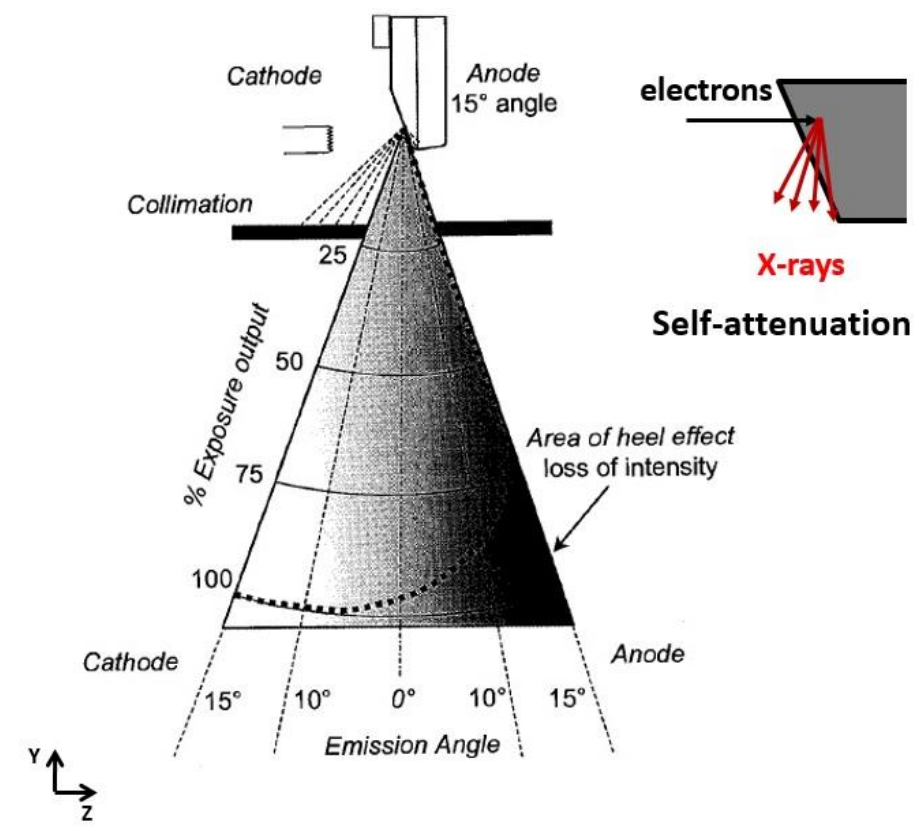

Figure 14. Schematic representation of the target self-attenuation (heel effect). It is caused by attenuation of the X-ray beam by the anode. Figure adapted from Bushberg (Bushberg, 2002)

\section{- Collimators}

In most $\mathrm{CT}$ devices, collimators have the function of shaping the X-ray beam into a fan or cone geometry depending on the kind of CT examination. Basically, CT systems incorporates two fixed collimators commonly called the pre-patient and post-patient collimators, as presented in Figure 15.b and Figure 15.c, which refine the beam shaping of the beam emitted from the tube portal into the desirable one. The first reduction of the X-ray beam is performed by the tube housing which has a lead shell that absorb the X-rays to just allow radiation emission through the tube portal. Since the tube housing avoids the emission of X-rays in any directions, it plays an important role in the radiation protection of patients and workers. This beam area reduction is done close to the tube focus and reduces the generated radiation roughly to the maximally anticipated beam for the given detector and geometry. A second step in the beam shaping is performed by a fixed collimator located after the bowtie filter, as presented in Figure 8.b and Figure 8.c. It allows adjustable aperture to the desired slice width or multiple slice widths. For instance, Figure 15.a illustrates the case of a fan X-ray beam which was shaped by the pre-patient collimator. This is placed as far from the focus as possible, for instance, close to the gantry housing aiming to minimize penumbra regions caused by the finite focus size (non-point focus), as presented in Figure 15.c. Figure 15.b and Figure 15.c show the characteristics of the dose profile on the axis of rotation (AOR) for a fan beam generated from a point focus and non-point focus, respectively. A point focus (or point 
focal spot) generates a rectangular dose profile on the AOR with width equal to the beam width, on the other hand, a non-point focus generates a trapezoidal dose profile with the length of its upper base is equal to the beam width (BW), as illustrated in Figure 15.b and Figure 15.c, respectively. A post-patient collimator is located close to the detectors as also presented in Figure 15.b and Figure 15.c. They reduce the incoming of the scattered radiation from the patient and therefore reduce the apparition of images artifacts.
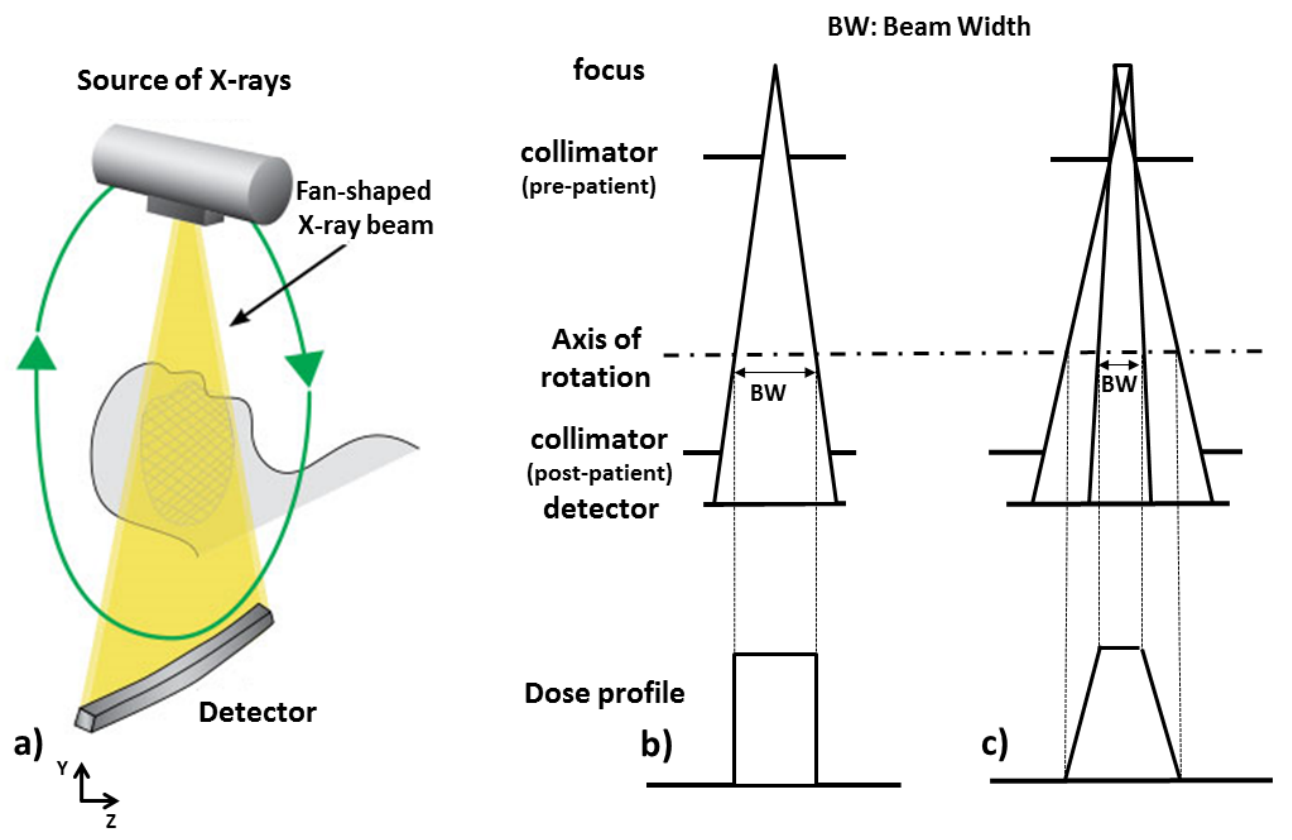

Figure 15. Beam geometry and profile characteristics, a) fan shaped X-ray beam, b) and c) dose profile from point and one-dimension focal spot. Adapted from Kalender (Kalender, 2011).

\section{- Flat and bowtie filter:}

As mentioned before, bremsstrahlung produces X-rays with energies ranging from few electronvolts up to a maximum value, $E_{\max }$, defined by the tube voltage. Low energy X-rays are not desirable because they have high probability of absorption by the human body, therefore, they have stronger contribution to the patient dose. In medical imaging, low energy quanta are responsible for the apparition of image artifacts due to the beam hardening effect ${ }^{\mathrm{o}}$. Therefore, in order to cut photons with low energies from the beam spectrum, additional filterers are incorporated in the X-ray tube portal. Although, the X-ray beam is filtered by the inherent filtration of the tube, additional filtration is added aiming to reduce more photons with low energies. A first step of filtration is performed by a flat shaped filter, which

\footnotetext{
${ }^{\circ}$ beam hardening is the physical process in which photons with low energies are cut from the spectrum resulting in a more penetrated beam with high average energy value.
} 
generally consists of a metal filter of some millimeters of thickness, which can visualized in Figure 8.b and Figure 8.c. Figure 16 illustrates the beam hardening performed by filters of $2.5 \mathrm{~mm}$ of $\mathrm{Al}$ or $0.2 \mathrm{~mm}$ of $\mathrm{Cu}$ on a spectrum produced by a tube voltages set to $120 \mathrm{kV}$, both presenting the characteristics lines $K_{\alpha}$ and $K_{\beta}\left(K_{\alpha 1}=59.3 \mathrm{keV}, K_{\alpha 2}=57.9 \mathrm{keV}, K_{\beta 1}=\right.$ $69.1 \mathrm{keV}, K_{\beta 2}=67.2 \mathrm{keV}$ ) of Tungsten, W. Moreover, X-ray spectrum with no filter and another one filtered by $750 \mathrm{~mm}$ of air are also presented. The X-ray beam with no filter contains fluorescence X-rays, characteristic of the target atomic number, as well as bremsstrahlung. In the figure, the spectra are in units of photons $/ \mathrm{mAs} . \mathrm{mm}^{2}$ at $0.75 \mathrm{~m}$. The spectra are in function of the photon energy in units of $\mathrm{keV}$. In the non-filtered spectrum and in the filtered by $750 \mathrm{~mm}$ of air, the characteristic lines, $L_{\alpha}$ and $L_{\beta}$, can be visualized, in contrast to the others two spectra filtered by $2.5 \mathrm{~mm}$ of $\mathrm{Al}$ and $0.2 \mathrm{~mm}$ of $\mathrm{Cu}$, where the $\mathrm{L}$ lines and low energy photons are absorbed producing a shifting of the center of the polychromatic X-ray to higher energies or harder radiation, this is the origin of what is called beam hardening. This pre-hardening of the radiation helps to reduce the apparition of beamhardening artifacts in the image, because today is standard to consider the X-ray to be monochromatic within the mathematical reconstruction process, for revision of artifact due to beam hardening, see Sect. 9.6.2 of Buzug (Buzug, 2008).

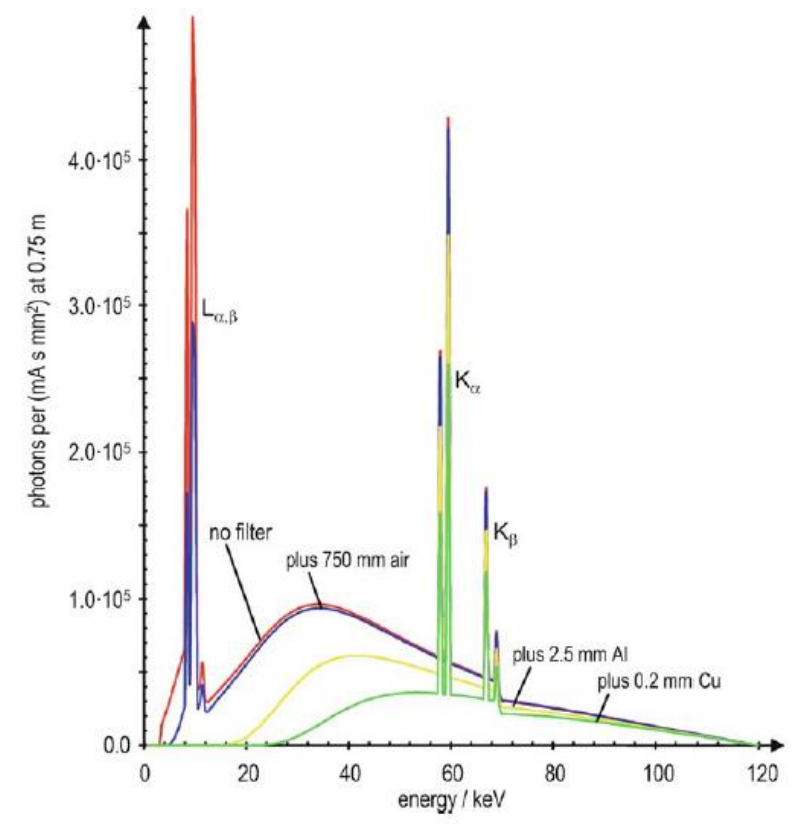

Figure 16. Beam hardening of an $\mathrm{X}$-ray spectrum produced by a tungsten anode (anode angle $10^{\circ}$, acceleration voltage $U_{a}=120 \mathrm{kV}$ ) due to flat shaped filters of $2.5 \mathrm{mmAl}$ and $0.2 \mathrm{mmCu}, 750 \mathrm{~mm}$ of air and also non-filtered spectrum is presented. The degree of absorption depends of the photon energy, in the figure, low energy photons are strongly absorbed. Figure adapted from Buzug (Buzug, 2008) (courtesy of B. David, Philips Research Labs.) 
In addition to the flat shaped filter, beam-shaped filters, so-called bowtie filters due to their shape, are also incorporated in the X-ray tube portal to provide an effective mean of dose utilization. Rather than the flat shaped filters, which reduced uniformly low energy photons from the beam along its extension, bowtie filter attenuation has a dependence of the polar angle and it aims to modulate the beam intensity in the plane perpendicular to the AOR, Axial plane, $x-y$, as shown in Figure 8.b and Figure 17. Bowtie filters absorb photons harder on the off-axis than the central axis to maintain a more uniform X-ray field at the detector (it compensates the roughly ellipsoidal cross-section of the human body), as illustrated in Figure 17 where the X-ray fluence is reduced as function of the fan angle (polar angle). In terms of dose reduction, bowtie filter allows reduction of periphery dose in patient without a big increment of the image noise. Also it reduces substantially the X-ray scattering effects, improving the signal-to-noise ratio in the image. Typically manufactures incorporates two sizes of bowtie filters (head and body filters), nevertheless, some of them offers filters based on size of the scanning object, incorporating small, medium, and large filters. Overall, proper selection of bowtie filters based on patient size and imaging needs may result in dose saving of up to $50 \%$ (Mahesh, 2009).

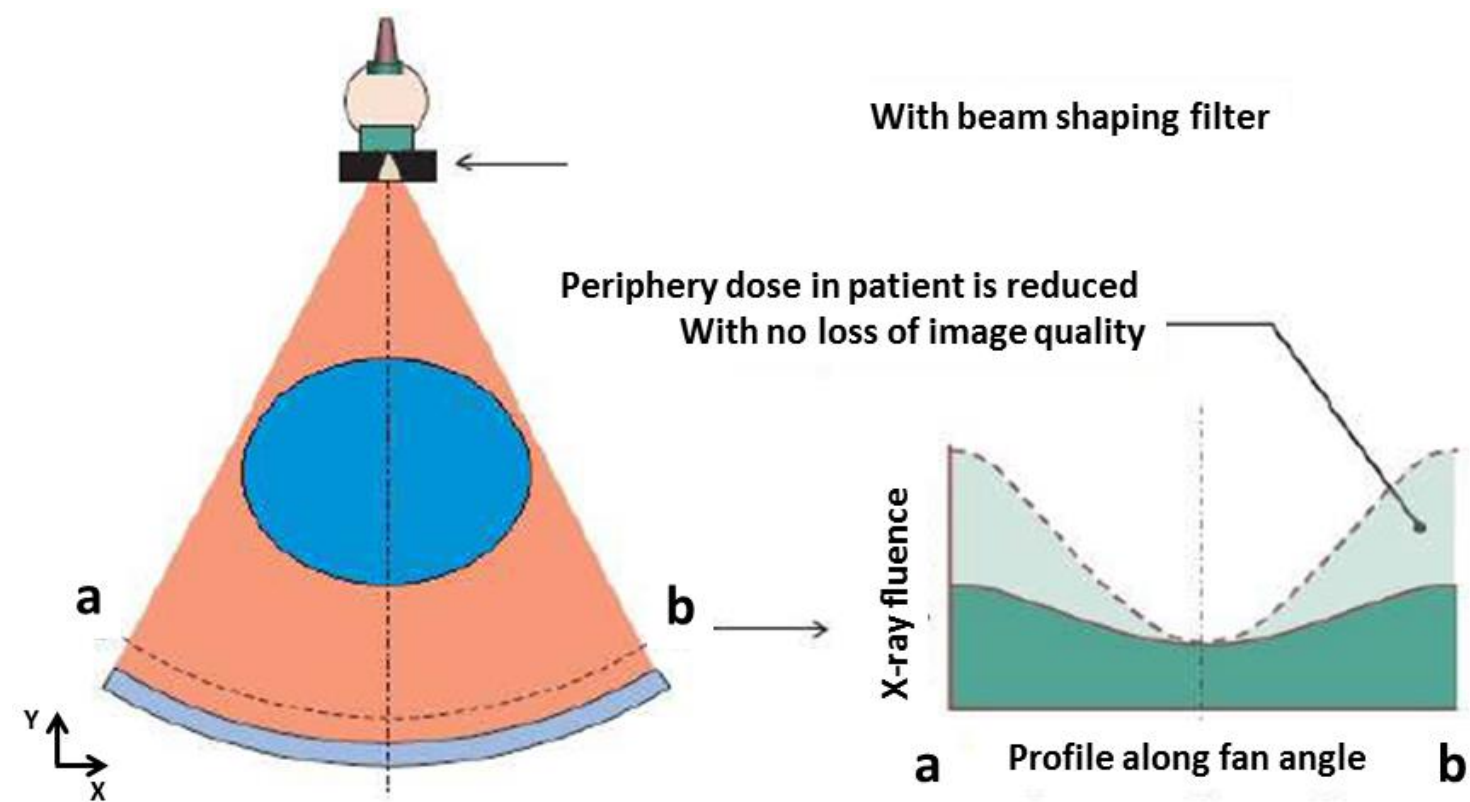

Figure 17. Impact of the bowtie filter attenuation on the $X$-ray fluence along fan angle. Bowtie filter reduces the incoming $X$-ray fluence (light-green profile) much higher at the periphery of the field than at the center due to its format, resulting in a more homogeneous photon fluence reaching the detection system (dark-green profile). Figure adapted from Bushberg et al. (Bushberg et al., 2011). 


\section{- CT table:}

The CT table or patient bed, shown in Figure 7.a, is an intrinsic component of CT scanners, which supports the patient during the CT examination. It can be lowered down to allow the patient to comfortably get on it and can be moved upwards again, according to necessity (ranges from 30 to $100 \mathrm{~cm}$ ). Patient bed is constructed to have precision of 1 to $2 \mathrm{~mm}$ for patient positioning and it move at constant or variable speed in and out of the gantry (ranges up to $200 \mathrm{~cm}$ ). Most Multiple-row detector CT (MDCT) tables can accommodate patient weights from 204 to $270 \mathrm{~kg}$. CT tables are commonly made up of carbon fiber materials that have the least X-ray absorption capabilities (Mahesh, 2009).

\subsubsection{Scan modes}

After that the patient is on the CT table to be examined by the CT scanner, two common CT scan modes could be selected to perform the CT examination and they are described as follow. First, the sequential or "step and shoot" scan mode where after each scan the table is moved to the next position before imaging the next region. Figure 18.a shows a schematic representation of the sequential scan mode in which there is no table movement during data acquisition, as well as, trying a minimum inter-scan delay between each slice as the table is moved to the next location. Certain cranial and cardiac CT scans are performed using the sequential scan mode. These days, CT angiography scans are doing with the sequential scan mode aiming for lower radiation doses (Mahesh, 2009).

Figure 18.b and Figure 18.c present schematic representations of the helical or spiral scan mode. In this case, the X-ray tube is continuously rotated with the examination table being moved linearly through the measuring field. This scan process produces data that take a helical or spiral path. This method offers the possibility of computing any number of slices retrospectively, so that an accurate three-dimensional rendering can be expected. In such a system, power supply to the X-ray tube and the signal transfer from the detector system is guaranteed, even though the imaging system continuously rotates. During helical scanning, the X-ray tube and the detectors rotate around the patient while the patient table moves at a constant speed along the $\mathrm{z}$ axis, creating a spiral exposure, as illustrated in both Figure 18.b and Figure 18.c. As the scanning is now helical, the data has to be interpolated to reconstruct each planar section. 

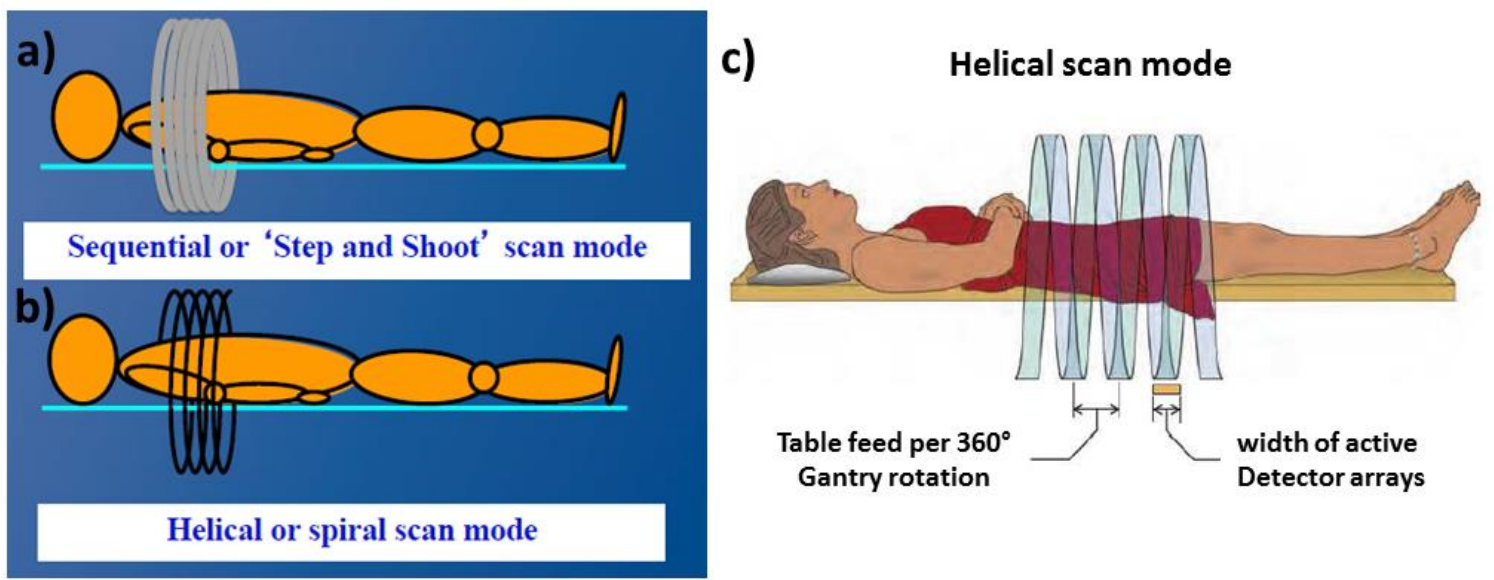

Figure 18. a) Sequential and b) helical CT scan mode. Figures adapted from Mahesh (Mahesh, 2009). c) Schematic representation of the helical scan mode showing the nominal collimated beam width (width of active detector arrays) and the table feed per rotation of the gantry. Figure adapted from Bushberg et al.

(Bushberg et al., 2011).

The relative motion of the table per tube rotation is described by the dimensionless parameter pitch, $b$, defined in Equation (11):

$$
p=\frac{b}{n T}
$$

In this equation, $b$ is the table feed per tube rotation, $n T$ is the nominal collimated beam width, $n$ is the number of detector rows and $T$ is the detector width. This definition is appropriated for single slice $(n=1)$ as well as for multiple slice $(n>1)$ scans. For example, a 16-slice scan $(n=16)$ with detector width $T=1.25 \mathrm{~mm}$ and table feed $b=5 \mathrm{~mm}$ has a pitch of $p=0.25$. Pitch $p=1.0$ corresponds to contiguous axial CT (Figure 19, middle). For the same tube current-time product value, pitch $p<1.0$ results in overscanning and hence higher radiation dose to the patient, but with improved image quality, compared to a scan performed with $p=1.0$ (Figure 19, top). On the other hand pitch $p>1.0$ represents underscanning and results in lower radiation dose (Figure 19, bottom), but the resultant images may contain reconstruction artifacts. 


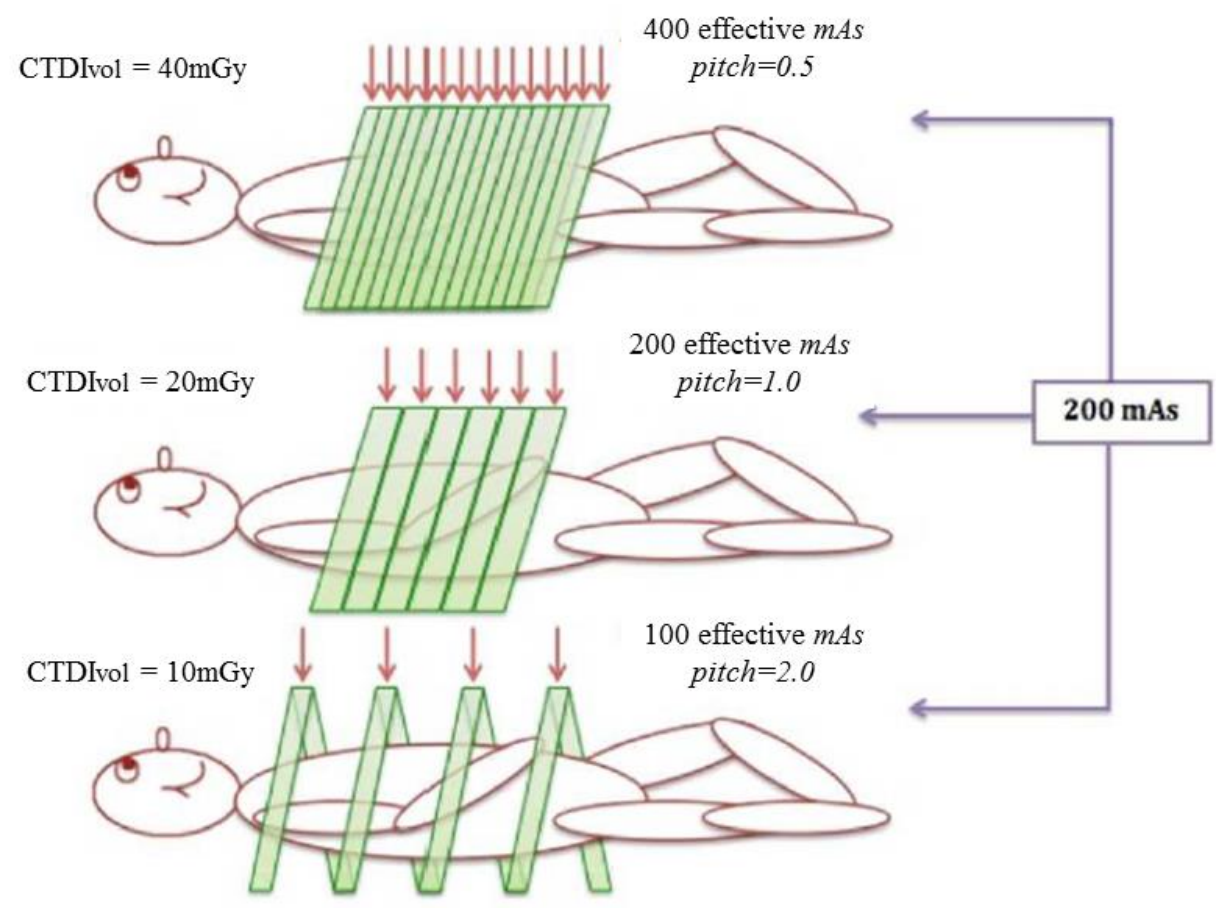

Figure 19. X-ray beam trajectories around the patient for different pitch values. $p<1$ results in overscanning (top), for pitch $p=1$ there is no overlap of the radiation beam path, and no uncovered regions, for pitch $p>1$ some regions may be uncovered due to underscanning. (source: Hypermedia MS).

\subsection{Current CTDI method for CT Dosimetry}

One of the main concerns in CT procedures is the quantification of the absorbed dose during a CT examination. Since a direct measurement of the patient absorbed dose is complex, alternative methods have been proposed in order to assess the patient dose indirectly. At the beginning of 80's, the Computed Tomography Dose Index, CTDI, was introduced by Shope et al. (Shope et al., 1981) in order to quantify the radiation output from a series of adjacent scans (sequential scans). With the advent of Multiple-row Detector CT, MDCT, introduced in 1998 (Kalender, 2011), the Volumetric Computed Tomography Dose Index, $C T D I_{v o l}$, was introduced to include the effect of "pitch" (table increment) on dose tracking (IEC, 2001). Helical protocols implying the scanning with continuous table motion and large scan lengths boost the definition of the Dose Length Product, DLP. This section presents in detail the definition and characteristics of the aforementioned CT metrics.

In the late of 1970 s, a common CT examination was composed by a number of continuous single axial scans spaced between them by a specific distances in the order of the selected nominal slice thickness (see, section 2.1.3 for explanation of sequential scan mode). As the number of $\mathrm{CT}$ examinations were increasing, many reports about the radiation 
exposure or dose delivered by CT systems have appeared which had described a series of measurement methods to characterize the radiation delivered by CT. Basically, these methods perform a measurement of the dose profile along the central axis or an axis parallel to the central axis of a PMMA cylindrical phantom for a single axial scan. Metrics such as the maximum dose value or the integral of the dose profile were taken into account to be the descriptor of the dose delivered by a CT examination (Shope et al., 1981), but, they are not sufficient to describe adequately the CT mode of operation. A practical method for dose description in CT was published in 1981 by a US Food and Drug Administration, FDA, group lead by Shope (Shope et al., 1981), who refined the mathematical formalism previously introduced by Jucius and Kambic and Suzuki and Suzuki (Jucius and Kambic, 1977, Suzuki and Suzuki, 1978). The method proposed by Shope et al. uses the Computed Tomography Dose Index, CTDI, as the dose descriptor for CT procedures consisting of a series of axial scans, as stated by Shope et al. "CTDI is a method for describing absorbed dose delivered by $X$-ray transmission computed tomography, which provides a mean to characterize the doses resulting from CT procedures consisting of a series of adjacent scans" (Shope et al., 1981). Formally, CTDI is defined as the integral of a single-scan dose profile, $f(z)$, along a line perpendicular to the tomographic section of a PMMA cylindrical phantom at a given point $(x, y)$ in the axial plane (see, Figure $8 . a)$ divided by $T$, the nominal slice thickness as stated by the manufacturer or selected by the CT system operator. Accordingly, CTDI is given by Equation (12) and often quoted in units of milligray (mGy).

$$
C T D I=\frac{1}{T} \int_{-\infty}^{\infty} f(z) d z
$$

As can be seen from Equation (12), CTDI represents a useful methodology to characterize radiation dose for multiple axial scans since only the single dose profile, $f(z)$, is needed for CTDI computation. Shope et al. determined the single scan dose profile by fitting the sum of two Gaussian functions to dose data obtained using thermoluminescent dosimeters, TLD. Although the use of TLD for CT dosimetry was frequently applied at the beginning of CT dosimetry, the use of pencil ion chamber (Jucius and Kambic, 1977, Suzuki and Suzuki, 1978) in the late 1970's presented advantages such as high sensitivity and dynamic ranges, quantitative measurement, the ability to measure internal and surface exposures, and immediate readings, yet at the same time the use of ion-chamber produced lack of information about the dose profile and requirement of a special chamber (pencil ion chamber). As defined by the AAPM Diagnostic Radiology Committee Task Force on CT Scanner Phantoms 
(AAPM, 1977) and adopted by the FDA in 1984 (FDA, 1984), the phantom used for CT dosimetry has cylindrical shape with 14 or $15 \mathrm{~cm}$ length and 16 or $32 \mathrm{~cm}$ diameters to represent the average head and body cross sections of an adult patient, respectively. For preprogramed protocols applied in pediatric exams, the $16 \mathrm{~cm}$ diameter phantom may refer to a child's torso, but this convention is not standardized across CT models of all manufactures (AAPM, 2010). Polymethyl Methacrylate (PMMA) is used as phantom material.

CTDI became the standard metric for CT dosimetry and, for practical purposes, a restriction in the integration interval was established. Despite the FDA establishment of the $C T D I_{F D A}$ with integration limits of $\pm 7 T$, where $T$ is the nominal beam width ${ }^{\mathrm{p}}$, the quantity $C T D I_{100}$ became the standard. This restricted definition of CTDI was introduced by Leitz et al. (Leitz et al., 1995) in 1995, which integrate the single dose profile from $-50 \mathrm{~mm}$ to $+50 \mathrm{~mm}$ centered on the peak of the physical dose profile. The work of Leitz et al. aimed to represent 'dose' for a national survey in Sweden and it was based on a $100 \mathrm{~mm}$ long pencil chamber measurement inside PMMA cylindrical phantoms. The effective patient doses calculated by Leitz et al (Leitz et al., 1995) were compared with Monte Carlo calculations and showed satisfactory agreement. Equation (13) presents the mathematical definitions of the $C T D I_{100}$, where $T$ is the nominal beam width.

$$
C^{2} D I_{100}=\frac{1}{T} \int_{-50 \mathrm{~mm}}^{50 \mathrm{~mm}} f(z) d z
$$

Figure 20 is a schematic representation of the methodology used to measure the $C T D I_{100}$ along the central and peripheral axes of the cylindrical PMMA phantom, where a hole at center and four holes at peripheral positions are used to put the $10 \mathrm{~cm}$ length ionization chamber.

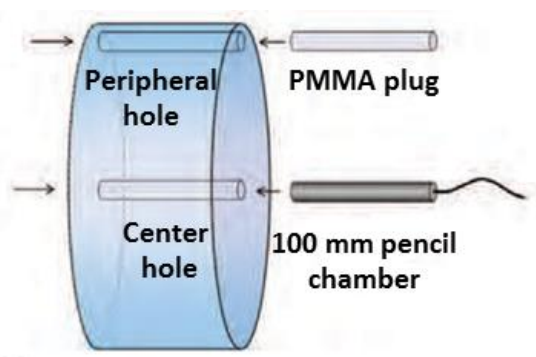

Figure 20. Schematic representation of the $C T D I$ phantom used to measure the $C T D I_{100}$. The holes at center and peripheral positions are used to put the $10 \mathrm{~cm}$ pencil ionization chamber to measure the center and peripheral $C T D I_{100}$, respectively. Figures adapted from AAPM report 204 (AAPM, 2011).

\footnotetext{
${ }^{\mathrm{p}}$ this metric had an implicit practical inconvenient due to the dependence of the integration limits with the nominal beam width which become non-practical the measurement of dose profile for high values of $\mathrm{T}$.
} 
$C T D I_{100}$ method was widely adopted as the most practical indicator of dose delivered by CT scanners $(\mathrm{EC}, 1998)$. Since the $C T D I_{100}$ has different values for central and peripheral phantom axes, the new index weighted-CTDI, $C T D I_{w}$, was introduced aiming to have a single $C T D I_{100}$ number (IEC, 2004, EC, 1998). This weighted $C T D I_{100}$ value led to an approximate 'weighted average' dose across the central scan plane at $\mathrm{z}=0$, if the total dose profile is centered at this point. $C T D I_{100}$ depends on scanner geometry, collimation and beam prefiltration, as well as on X-ray tube voltage, X-ray tube current, and gantry rotation time. Equation (14) presents the mathematical representation for the $C T D I_{w}$, it is defined in milligray (mGy):

$$
C T D I_{w}=(1 / 3) C T D I_{100, c}+(2 / 3) C T D I_{100, p}
$$

Where, $C T D I_{100, c}$ is the index measured along the central axis and $C T D I_{100, p}$ is the index measured along the peripheral axis. The weighting factors were assumed ad hoc as linear variation of $C T D I_{100}$ from central to peripheral phantom axis (IAEA, 2011).

With the advent of MDCT in 1998, the normalization term in the CTDI 100 mathematical definition, Equation (13), was redefined to $n T$ which is the active detector length as projected back to scanner AOR and represents the total available scan width for reconstruction. In this case, $n$ represents the number of tomographic sections imaged in a single axial scan. This is equal to the number of data channels used in a particular scan. The value of $n$ is less than or equal to the maximum number of data channels available on the CT detector system. $T$ is the nominal width of the tomographic section along the $\mathrm{z}$-axis imaged by one data channel (note that, in this case, the definition of $T$ was changed) (see, Section 2.1.3). Thus, Equation (13) was redefined to Equation (15) as follow:

$$
C T D I_{100}=\frac{1}{n T} \int_{-50 \mathrm{~mm}}^{50 \mathrm{~mm}} f(z) d z
$$

The introduction of the helical CT (see, Section 2.1.3) by Kalender (Kalender et al., 1989) brought a new need to cover the movement of patient table during the CT examination. Therefore, the $C T D I_{w}$ was modified by the IEC (IEC, 2001) to include the CTDI $I_{\text {vol }}$ considering the effect of "pitch", $p$, on dose as shown in Equation (16):

$$
C T D I_{\text {vol }}=p^{-1} C T D I_{w}
$$

Where, $p=b / n T$ is applied for both helical and sequential scans. $b$ is the table displacement per gantry rotation (for helical scan mode) or the midpoint-to-midpoint spacing 
between successive scans (for sequential scan mode). The index $C T D I_{\text {vol }}$ represented an improvement since it eliminates $n T$ in the formulation so that only the inverse of the table increment per rotation, $b^{-1}$, matters (Spokas, 1982, Dixon, 2003). This quantity is displayed on the console of current CT scanners. $C T D I_{v o l}$ estimates the average radiation dose within the middle portion of the irradiated volume of CT acquisition. Therefore, CTDI $I_{v o l}$ does not represent a volume average as its subscript might suggest. However, it still represents the planar average dose over the central scan plane (at $\mathrm{z}=0$ ) for a $100 \mathrm{~mm}$ scan length. Its basis is still $C T D I_{100}$ which is hidden in the formulation. An important issue to be noted is that when $b$ tends to be zero, $(b \rightarrow 0), C T D I_{v o l}$ tends to be infinite, however, this is meaningless since the actual dose remains finite, therefore, $C T D I_{v o l}$ does not applied to static table (or stationary phantom) modes (Dixon and Boone, 2014).

$C T D I_{v o l}$ does not indicate the total energy deposited in the scan volume, its value remains unchanged whether $1 \mathrm{~cm}$ or $100 \mathrm{~cm}$ of patient anatomy is scanned. Therefore, for better representation of the overall energy (or dose) deposition, an integration over the scan length is needed, resulting in the new descriptor, Dose Length Product, DLP $=L x C T D I_{\text {vol }}$. Note that in this case, the $D L P$ does not depend on $b$ since, $L=N b$ and $C T D I_{v o l}$ is proportional to $b^{-1}$. Thus $b$ is canceled in the product, and $D L P$ really depend only on the number of rotations $N$ or the total $m A s$. Therefore, a change in the technique (mAs/rotation) affects the $C T D I_{v o l}$, while a change in acquisition length (at the same technique) is reflected in the DLP. Figure 21 shows the schematic evolution of CT dose descriptors based on the CTDI method, their evolution depended on the incorporation of new CT characteristics.

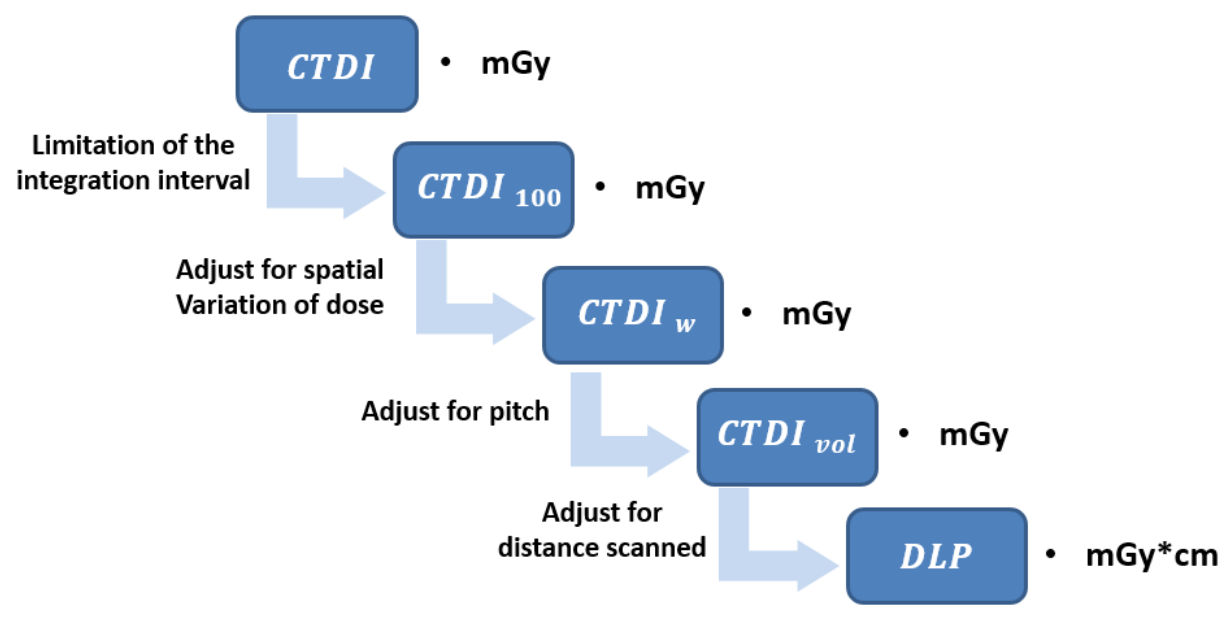

Figure 21. CT dose descriptors based on the CTDI index. They are currently used for CT dosimetry evaluation. 
It is important to note that the core of the CTDI paradigm is the dose profile produced in a single scanning, as presented in Equation (12). The following section presents the analytical formalism for the single dose profiles, which will be used to validate the dose profiles computed from MC simulation.

\subsection{CT Dose Profile formalism}

Radiation dose distribution in CT can be defined considering the spatial distribution of the deposited energy inside of a CTDI phantom produced due to the interaction of the X-ray beam with the phantom material. For CT dosimetry purposes, one-dimensional dose distributions are commonly measured or computed along the central or peripheral axes of the CTDI phantoms, which are named dose profiles. In analytical terms, a dose profile function is generally expressed as a function of the z-coordinate of a three-dimensional coordinate system in which the z-axis is aligned with the Axis of Rotation (AOR) of the CT gantry. For an analytical or physical study, it is instructive to understand a dose profile produced by a single axial-rotation scan as composed by two components. Primary component, $f_{p}(z)$, which is the deposited energy from the interaction between the primary beam with the phantom material on an axis of interest and a scatter component, $f_{s}(z)$, which is the deposited energy on an axis of interest delivered by the secondary radiation produced by the interaction of the primary radiation with the phantom material. Both components and a total dose profile from a single axial-rotation scan are illustrated in Figure 22. Therefore, analytically the dose profile is expressed as the sum of the primary, $f_{p}(z)$, and scatter, $f_{s}(z)$, components as formulated in Equation (17).

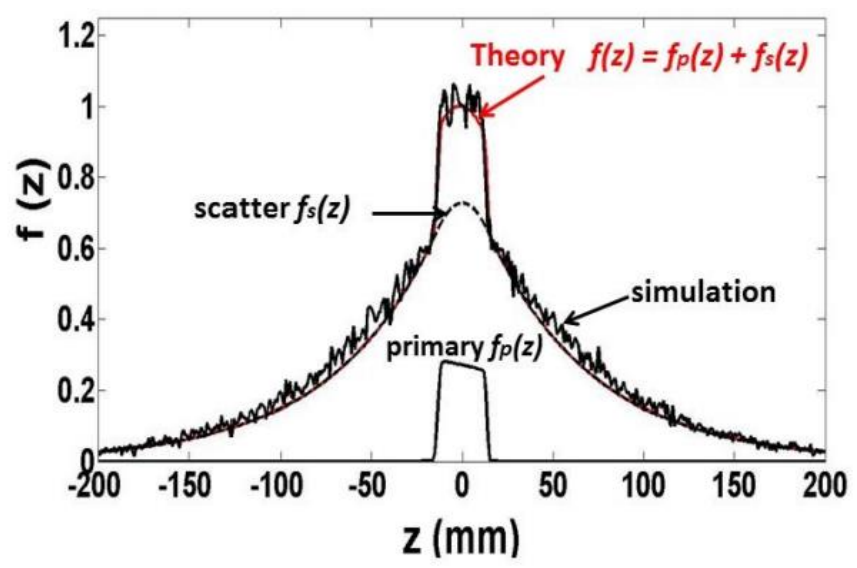

Figure 22. The total dose profile $f(z)$ is the sum of a primary $f_{p}(z)$, and a scatter $f_{s}(z)$ component. Figure also shows a comparison with a simulated one. 


$$
f(z)=f_{p}(z)+f_{s}(z)
$$

Analytical formulations have been developed to study the influence on the dose profile by the CT geometry, CT radiation output and novel dose reduction strategies (Gagne, 1989, Dixon et al., 2005, Dixon and Boone, 2013). A pioneer work about an analytical formulation for the dose profile was published in 1989 by Robert Gagne (Gagne, 1989). This work proposed an analytical formalism for the dose profiles based on a simplified CT geometrical model, which showed the functional dependence of the dose profile with the shape and size of the X-ray tube focal spot and the position and aperture of the CT pre-patient collimator (Costa, 1993, Gagne, 1989). Nevertheless, the Gagne's analytical model disregarded the effects on the dose profile by the target self-attenuation (heel effect), the anode angle (penumbra asymmetry) and the X-ray energy spectrum (Dixon et al., 2005). With the advent of MDCT X-ray systems and the use of broader X-ray beams, $160 \mathrm{~mm}$ of beam aperture for a 320-detector row $\mathrm{CT}^{\mathrm{q}}$, effects on the penumbra due to the anode angle, distortions on the primary dose profile due to heel effect and the influence of the X-ray energy spectrum have become more important. A more realistic analytical formulation for the dose profile, which transcends all of the aforementioned limitations of the Gagne model, was proposed by Dixon et al (Dixon et al., 2005), which included the effects of the target self-attenuation, the anode angle and the X-ray energy spectrum. Dixon et al's formulation extended the analytical formalism for CT dose profiles to peripheral axis (Gagne's model only apply for regions near to the central axis) located at $10 \mathrm{~mm}$ below the surface of a CTDI phantom. It was performed for completeness, improving the information about that peripheral dose profile and to implement the use of a small ion-chamber of $1 \mathrm{~cm}$ length and $0.6 \mathrm{~cm}^{3}$ volume as a new methodology for dosimetry evaluation in CT (Dixon, 2003).

The following sections present the analytical formalisms for the primary and scatter components of the dose profile. Section 2.3.1 presents the analytical formalism for the primary component represented along the central and peripheral axes of the CTDI phantom. Section 2.3.2 describes the derivation for the scatter component from the convolution between the scatter impulse response function (LSF) with the primary beam function. Section 2.3.3 presents the final analytical form for the total dose profile and Section 2.3.4 shows the analytical formulas for the cumulative dose profile produced by a CT helical examination.

\footnotetext{
${ }^{\mathrm{q}}$ For instance, the CT scanner Aquilion One introduced in 2007 by the Toshiba Medical Systems (Otawara-shi, Japan).
} 


\subsubsection{Primary Dose Profile}

The curve in Figure 23 represents a primary dose profile, $f_{p}(z)$, computed on AOR of the CT gantry without the influence of the CTDI phantom (dose profile free-in-air) with CT parameters setting to: beam width, $a=11.4 \mathrm{~mm}$, body bowtie filter and small focal spot. As mentioned before, a primary dose profile is affected by the anode angle and the heel effect. Therefore, the shape of the primary profile can be used to evaluate these CT device characteristics. In Figure 23, the initial (left) and final (right) portion of the curve are named penumbras and they are produced due to the focal spot is a non-point X-ray source. As observed, these penumbras are asymmetric between them which is consequence of the focal spot tilt. The heel effect, which is the X-ray non-uniform attenuation inside the anode, affects the X-ray fluence along the $\mathrm{z}$-direction, making that the deposited energy on the $\mathrm{z}$-axis not be uniform. Therefore, a decreasing intensity in the primary dose profile from left to right at its central region is observed, as also shown in Figure 23. Next, the analytic formulation of Dixon et al is described for the primary dose profile at the central and peripheral axes of a CTDI phantom. The formalism incorporates the effects of the anode angle, heel effect and the X-ray energy spectrum as mentioned by Dixon et al (Dixon et al., 2005).

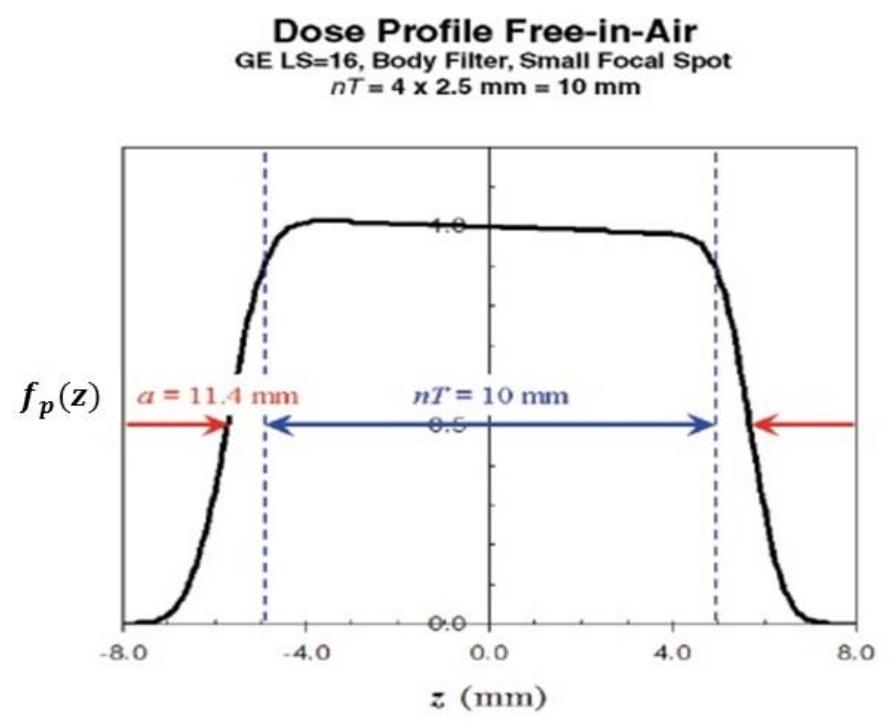

Figure 23. Analytical primary profile $f_{p}(z)$, computed on the axis of rotation, AOR. The beam width $a$ is $14 \%$ broader than the nominal one $n T$. Adapter from AAPM report 111 (AAPM, 2010)

Firstly, it is instructive to start the analytic derivation of $f_{p}(z)$ understanding the simplified CT geometry illustrated in Figure 24. It shows basic CT elements such as the focal spot tilted by the angle, $\alpha$, the pre-patient collimator, the gantry AOR, as well as, the focal spot to collimator distances, $F_{c}$, and the focal spot to AOR distances, $F$. In this model, the 
focal spot is a two-dimensional region on surface of the target from which the X-ray radiation is emitted. As explained in Section 2.1.2, the electron beam impinges the target and produces $\mathrm{X}$-ray radiation, which is assumed in this simplified model, emitted only from the focal spot (two-dimensional region). In Figure 24, the dashed lines from the center of the focal spot to the AOR are plotted to define the projection, $a$, of the collimator aperture, $w$, on the AOR. In the same figure, the continuous lines from both edges of the focal spot to the AOR are plotted to define the penumbra width denoted by, $c$. Geometrically, the differences in the penumbra sizes at left and right is determined by the target angle.

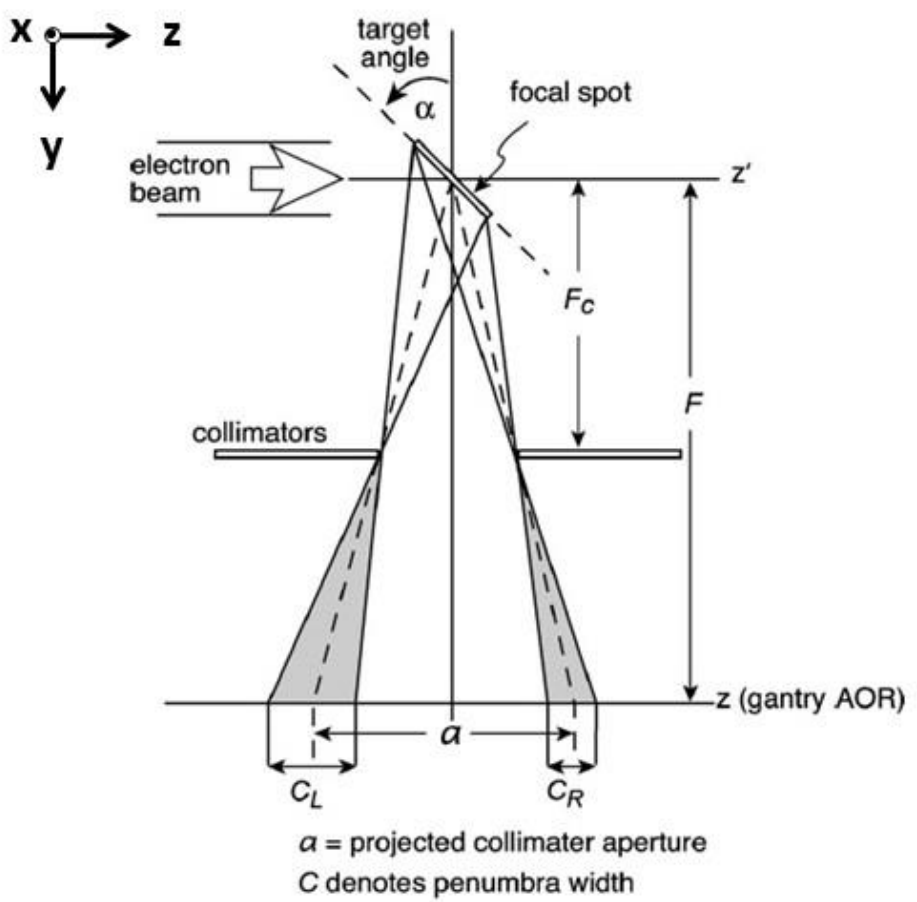

Figure 24 Simplified geometrical model of a CT system. Figure adapted from (Dixon et al., 2005).

Summarizing, in Figure 24, a represent the collimator aperture projected onto the gantry AOR from the central point of the focal spot, and $c_{L}$ and $c_{R}$ represent the penumbra width at left and right sides, respectively. Figure 24 also shows the target angle, $\alpha$, determined with respect to an axis perpendicular to the AOR. Moreover, the ratio between the focal spot to AOR distance, $F$, and the focal spot to collimator distance, $F_{c}$, is named the magnification of the CT system, $M=F / F_{c}$. The projection, $a$, is proportional to the collimator aperture, $w$, by the magnification factor, $M$, as $a=M . w$. The optical length, $c^{\prime}$, defined as the apparent length of the focal spot for a viewer on the AOR (see, Figure 13.a) produces an asymmetric 
penumbra $c$ on the AOR, with the relationship $c / c^{\prime}=M-1$. When $\alpha \rightarrow 90^{\circ}$, the model presented in the Figure 24 becomes a Flat Model and the penumbra and the dose profile become symmetric. The beam profile integral, which is the area of the trapezoid in Figure 24, depends solely of the projection collimator aperture, $a$, and the peak dose, $A_{0}$, and it is independent of the focal spot size or angle.

A more realistic CT geometrical model is illustrated in Figure 25. In this model, the focal spot, which its relative size has been highly exaggerated for clarity, is a small distance beneath the actual target surface (represented by the dashed line). This set up help to implement the target self-attenuation in the analytical formulation of the primary dose profile. Three coordinate systems can be identified in Figure 25. At left, the coordinate system, $\left(x^{\prime}, y^{\prime}, z^{\prime}\right)$, is associated to the $\mathrm{x}$-ray tube target. At medium, the pre-patient collimator is associated to the $\left(x_{c}, y_{c}, z_{c}\right)$ coordinate system and at right, the coordinate system, $(x, y, z)$, is associated to a cylindrical phantom of radius, $R$. CT systems are manufactured such as the target and gantry axis of rotation and the collimator plane are parallel, therefore in the figure, $z^{\prime}, z_{c}$ and $z$ are parallel axes. The planar focal spot (two-dimensional), represented in Figure 25 , is on the $s-x^{\prime}$ plane, in which the coordinate, $s$, is on the length, $l$, of the focal spot (in this case, $l$, represent the biggest dimension of the 2-dimensional focal spot) and $x^{\prime}$ is perpendicular to the page. Dixon et al start the derivation of $f_{p}(z)$ defining the fluence rate function, $\dot{S}_{A}\left(s, x^{\prime}\right)$, with units of photons $/ \mathrm{cm}^{2} / \mathrm{s}$ as the emission intensity distribution per unit time. This intensity represents the number of photons emitted by the focal spot at $\left(s, x^{\prime}\right)$ $\left(\left(s, x^{\prime}\right)\right.$ are the coordinates of a point on the focal spot surface, on the $s-x^{\prime}$ plane $)$ per unit area and unit time and where the photon energy distribution correspond to the bremsstrahlung emission of the electron-target collision. 


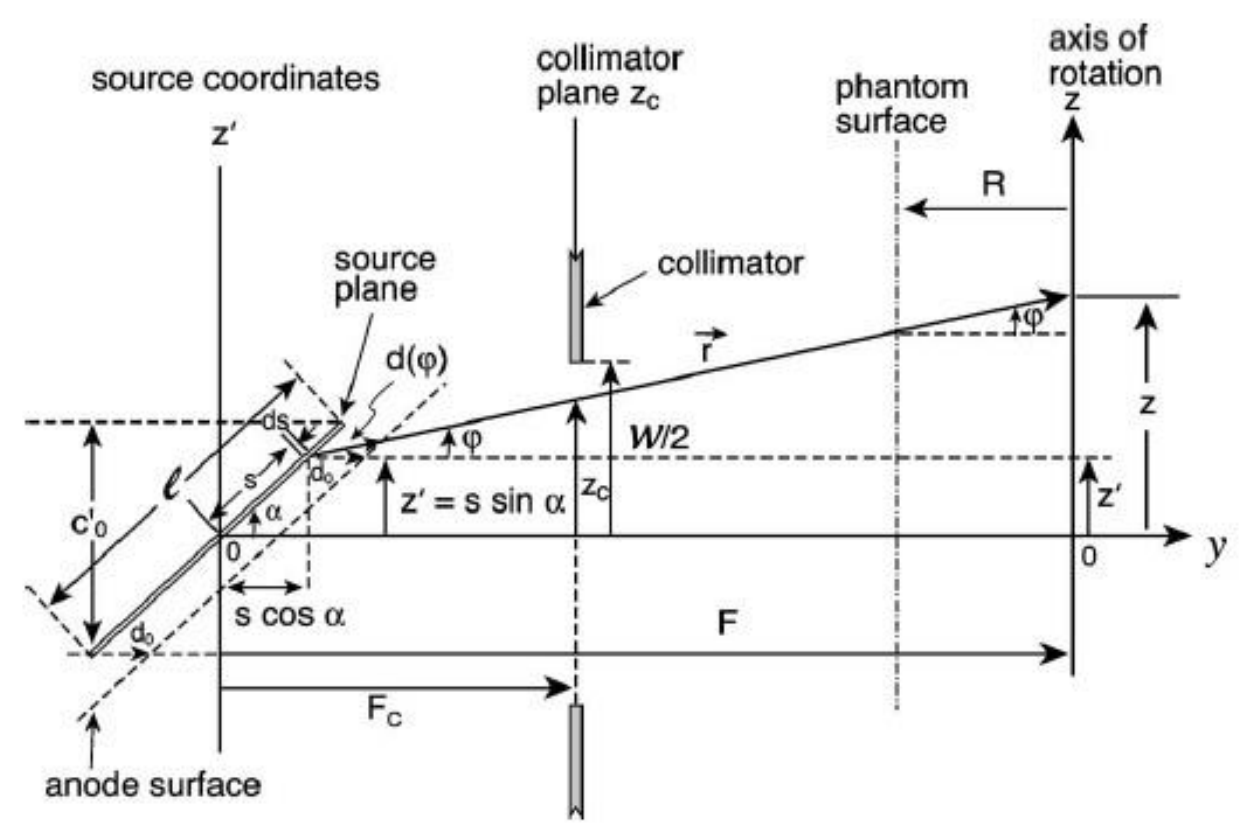

Figure 25 Geometry for the generalized primary beam model. Figure adapted from (Dixon et al., 2005).

Using geometrical assumptions, the two-dimensional function, $\dot{S}_{A}\left(s, x^{\prime}\right)$, can be reduced to a 1-dimensional one since, in CT machines, the actual focal spot dimension along the axis, $x^{\prime}$, is around $\approx 1 \mathrm{~mm}$. Therefore, it can be considered a point. For instance, in the GE Lightspeed family scanners, the focal spot to AOR distance is $F=541 \mathrm{~mm}$, then, $F \gg 1 \mathrm{~mm}$, and, a viewer on AOR sees the focal spot dimension along $x^{\prime}$ approximately as a point. The focal spot can be modelled as a 1-dimensional radiation source (line source) having an intensity per unit length along, $s$, given by the equation (18).

$$
\int_{-\infty}^{\infty} \dot{S}_{A}\left(s, x^{\prime}\right) d x^{\prime} \equiv \dot{S} \frac{1}{l} g\left(\frac{s}{l}\right)=\left\{\begin{array}{c}
\text { number photons } / \text { s emitted } \\
\text { per unit length at } s
\end{array}\right\}
$$

In equation (18), $\dot{S}$ is the total number of photons per unit of time (photons/s) emitted by the focal spot, which also contains the emitted photons across the focal spot dimension on the $x^{\prime}$ axis. The term, $\frac{1}{l} g\left(\frac{s}{l}\right)$, represents the photon intensity distribution function, along the $s$ axis in units $(1 / \mathrm{cm})$, normalized to unit area over $(-\infty,+\infty)$. In particular cases, if $g\left(\frac{s}{l}\right)$ represents a Gaussian or "rectangular" distribution, modelled as: $\exp \left[-\pi\left(\frac{s^{2}}{l^{2}}\right)\right]$ or $\Pi\left(\frac{s}{l}\right)$, respectively, $l$ is a representative parameter of the full width at half maximum (FWHM) of the corresponding function. As mentioned before, the photon energy distribution emitted by a "point" on the focal spot surface follows the bremsstrahlung emission, which is the electromagnetic radiation emitted by a charged particle (i.e. electrons) when it is deflected by 
an electromagnetic field (nucleus field), (Attix, 1986). In contrast to the fluorescence emission, which presents an isotropic emission in an electron-target interaction (neglecting attenuation of $\mathrm{x}$-rays in escaping the target), the bremsstrahlung radiation is emitted anisotropically, tending to reach a major intensity in the electron's direction with increasing energy (Attix, 1986). At this point, Dixon et al simplified the analytical derivation of the primary dose profile considering a small $\left(\approx 1^{\circ}-2^{\circ}\right)$ angular interval, in the slice-width $(\mathrm{z})$ direction, in which the angular dependence of the bremsstrahlung emission can be neglected. This simplification is valid for beam width up to $40 \mathrm{~mm}$ on the AOR. Therefore, the photon emission function can be written only with its energy's dependence, as: $\dot{S}=\dot{S}(E)$.

In order to establish the dose contribution at a point $z$ on the AOR due to a point $s$ on the focal spot, a straight line from $s$ to $z$, represented by the vector, $\vec{r}$, which crosses the collimator with aperture, $w$, is defined as shown in Figure 25. To define a differential equation for the incremental (scalar) photon flux density, $\Phi$, at $z$, contributions of photons from $d s$ at $s$ having energy in $d E$ at $E$ are considered and it is defined the equation (19), that is, a second-order differential equation in which two new factors appears.

$$
d^{2} \Phi=\frac{\dot{S}(E) d E}{4 \pi r^{2}} e^{-\mu(E) d(\varphi)} \frac{1}{l} g\left(\frac{s}{l}\right) d s
$$

In equation (19), the term $4 \pi r^{2}$ represents the surface area of a sphere with radius, $r$. This term allows to introduce the isotropic divergence photon emission (inverse-square law) from a point on the focal spot. In other words, the focal spot emits the total number of photons $\dot{S}$ in all direction without preference. The exponential term, $e^{-\mu(E) d(\varphi)}$, (where, $\mu(E)$ is the linear attenuation coefficient of the target material, and $d(\varphi)$, is the path length in the target) represents the target self-attenuation. In order to put in evidence the term that produces the heel effect, the target path length $d(\varphi)$ is expressed as follow, $d(\varphi)=d_{0}+\Delta d(\varphi)$, where, the constant value, $d_{0}$, is the distance beneath the actual target surface (dashed line) where the focal spot is located, it is defined parallel to the $y^{\prime}$ axis, as shown in Figure 25. The term, $\Delta d(\varphi)$, which can be positive or negative, models the difference of photon path in the target with respect to $d_{0}$. Associating the exponential factor involving $d_{0}$ with the spectral emission term, $\dot{S}(E)$, such that $\dot{S}_{0}(E)=\dot{S}(E) e^{-\mu(E) d_{0}}$ now represents the thick target spectrum emitted in the central ray direction, $y-y^{\prime}$, when $\varphi=0$. So, the remaining term $e^{-\mu(E) \Delta d(\varphi)}$ models the non-uniform attenuation inside the anode and it will express the heel effect. 
A constraint may be applied to the photon flux density, $\Phi$, in order to model the collimator's transmission. The function, $\Pi\left(\frac{z_{c}}{w}\right)$, which represents the probability of the photon emitted in the focal spot reaches the point $z$ on the AOR, is chosen. The coordinate $z_{c}$ corresponds to an axis related to the collimator parallel to AOR. The attenuation factors for the filter and for the phantom are $e^{-\mu_{f}(E) d_{f} / \cos (\varphi)}$ and $e^{-\mu_{p}(E) R / \cos (\varphi)}$, respectively. Where $d_{f}$ represents the central ray filter thickness and $R$ is the phantom radius. Therefore, the primary beam dose rate is shown in equation (20).

$$
\begin{aligned}
& \dot{f}_{p}(z) \\
& =\int_{E} \frac{k(E) \dot{S}_{0}(E) d E}{4 \pi F^{2}}\left\{\int_{s} \frac{1}{l} g\left(\frac{S}{l}\right) e^{-\mu(E) \Delta d(\varphi)} e^{-\mu_{f}(E) d_{f} / \cos (\varphi)} e^{-\mu_{p}(E) R / \cos (\varphi)} \frac{\cos ^{2}(\varphi)}{\left(1-\frac{s}{F} \cos (\alpha)\right)^{2}} \Pi\left(\frac{z_{c}}{w}\right) d s\right\}
\end{aligned}
$$

Where, $k(E)=E\left(\mu_{e n} / \rho\right)$ represents the fluence-to-dose conversion coefficient. The last equation was obtained considering: $r \cos (\varphi)=F-s \cos (\alpha)$. It was used to eliminate the dependence on $r$. Note that $z_{c}$ and $\varphi$ are both function of $s$, connected by the constraint equation (21):

$$
\tan (\varphi)=\frac{z-s \sin \alpha}{F-s \cos \alpha}=\frac{z_{c}-s \sin \alpha}{F_{c}-s \cos \alpha}
$$

The dose rate on the AOR depends over the phantom geometry for cylindrical phantoms. It turns independent of the beam rotation angle $(\theta=\omega t)$ and time. Therefore, we can obtain the dose profile on the AOR multiplying the equation (20) by the rotation time $\tau$.

Figure 26 shows the scheme to derive the primary dose spread function at the central axis of rotation, AOR. 


$$
\begin{aligned}
& \dot{S}_{A}\left(s, x^{\prime}\right) \rightarrow\left\{\text { emission intensity distribution }\left[\text { photons } / \mathrm{cm}^{2} / s\right]\right\} \\
& \downarrow \\
& \int_{-\infty}^{\infty} \dot{S}_{A}\left(s, x^{\prime}\right) d x^{\prime} \equiv \dot{S} \frac{1}{l} g\left(\frac{s}{l}\right) \rightarrow\left\{\begin{array}{c}
\text { number photons } / \text { s emitted } \\
\text { per unit length at } s
\end{array}\right\} \\
& d^{2} \Phi=\frac{\dot{S}(E) d E}{4 \pi r^{2}} e^{-\mu(E) d(\varphi)} \frac{1}{l} g\left(\frac{s}{l}\right) d s \rightarrow\left\{\begin{array}{c}
\text { incremetal photon flux density at "z" } \\
\text { contributted by phantom emitted from ds at s } \\
\text { having energy in } \mathrm{dE} \text { at } \mathrm{E} .
\end{array}\right\} \\
& \downarrow \quad\left[\begin{array}{c}
d(\varphi)=d_{0}+\Delta d(\varphi) / \dot{S}_{0}(E)=\dot{S}(E) e^{-\mu(E) d_{0}} \\
e^{-\mu_{f}(E) d_{f} / \cos (\varphi)} \rightarrow \text { filter attenuation } \\
e^{-\mu_{p}(E) R / \cos (\varphi)} \rightarrow \text { phatom attenuation } \\
\Pi\left(\frac{z_{c}}{w}\right) \rightarrow \text { probability of photons will reach a point at } z \\
k(E)=E\left(\mu_{e n} / \rho\right) \rightarrow \text { fluence to dose conversion factor }
\end{array}\right] \\
& \dot{f}_{p}(z)=\int_{E} \frac{k(E) \cdot \dot{S}_{0}(E) \cdot d E}{4 \pi \cdot F^{2}} \cdot\left\{\int_{s} \frac{1}{l} \cdot g\left(\frac{S}{l}\right) \cdot e^{-\mu(E) \Delta d(\varphi)} \cdot e^{-\mu_{f}(E) d_{f} / \cos (\varphi)} \cdot e^{-\mu_{p}(E) R / \cos (\varphi)} \cdot \frac{\cos ^{2}(\varphi)}{\left(1-s \cdot \cos \left(\frac{\alpha}{F}\right)\right)^{2}} \cdot \Pi\left(\frac{Z_{c}}{w}\right) \cdot d s\right\}
\end{aligned}
$$

Figure 26 Scheme to derive the primary dose profile at the central axis of rotation, AOR.

A set of approximations will be considered in order to obtain an analytical function for the primary dose profile along the AOR for a single slice.

- Small angle approximation, $\cos (\varphi)=1$ : Currently, for a single slice corresponding to a maximum beam width $T=40 \mathrm{~mm}$ ( $N \Delta T$ total active detector length), the maximum angle $\varphi$ will be, $\varphi_{\max }=2.4^{\circ}$, therefore, $\cos \varphi_{\max }=0.9991$. The approximation consists in setting $\cos \varphi=1$ with error of $0.1 \%$ for $T=20 \mathrm{~mm}$ and $0.3 \%$ for $T=40 \mathrm{~mm}$ (Dixon et al., 2005). This approximation overestimates the absorbed dose and implies that the phantom attenuation is uniform and the dose profile shape along the phantom axis has the same form in air, without phantom material. The small angle approximation allows to put out the filter and phantom attenuation terms of the source coordinate integral and put it into the energy integral. After the small angle approximation, the filter attenuation factor is absorbed into the spectral term, such that the modified spectrum, $\tilde{S}_{0}(E)=S_{0}(E) \cdot e^{-\mu_{f}(E) \cdot d_{f}}$, represents the bremsstrahlung spectrum on the central ray exiting the gantry port. This spectrum has not the contribution of low photon energy due to the filter attenuation. Therefore, the primary dose profile can be expressed as shown in the equation (22).

$$
\dot{f}_{p}(z) \cong \int_{E} \dot{A}_{0}(E) d E\left\{\int_{s} \frac{1}{l} g\left(\frac{s}{l}\right) e^{-\mu(E) \Delta d(\varphi)}\left(1-\frac{s}{F} \cos (\alpha)\right)^{-2} \Pi\left(\frac{z_{c}}{w}\right) d s\right\}
$$

Where the energy integrand term, $A_{0}(E)$, is defined in equation (23) as: 


$$
A_{0}(E)=\frac{k(E) \tilde{S}_{0}(E) e^{-\mu_{p}(E) R}}{4 \pi F^{2}}=\left\{\begin{array}{c}
\text { emitted dose per unit energy } \\
\text { at the central phantom axis }
\end{array}\right\}
$$

The term shown in equation (24) will be regardless because produce a small excursion in the penumbra regions at $z= \pm a / 2$ of $\pm 0.8 \%$ (Dixon et al., 2005), respectively, which would be barely noticeable in these high gradient regions. It will be therefore ignored.

$$
\left(1-\frac{s}{F} \cdot \cos \alpha\right)^{-2}
$$

For the larger beam widths $\left(a \geq 2 c_{0} \approx 5 m m\right), f_{p}(0)=A_{0}$.

- The heel effect factor: The photon fluence changes because of the self-attenuation inside the target. Both the photon intensity and photon energy distribution will be modified. Considering the empirical fit of heel effect slope set on $\mu d_{0}=0.28$, equation (25) and equation (26), as follow:

$$
\begin{gathered}
d(\varphi)=d_{0} \frac{\sin \alpha}{\sin (\alpha-\varphi)} \\
\tan (\varphi)=\frac{z-z^{\prime}}{F\left(1-z^{\prime} / z_{\alpha}\right)}
\end{gathered}
$$

The approximation of the point source will be possible with an error smaller than $0.2 \%$ and the exponential factor will become $\exp \left[-\mu \Delta d\left(\varphi_{0}\right)\right]$, where $\tan \left(\varphi_{0}\right)=z / F$, which is independent of the source coordinate $s$. Elsewhere, considering the small angle approximation, $\sin \varphi \cong \tan \varphi$, and $\cos \varphi \cong 1$, and using equation (25) and equation (26), we can obtain equation (27):

$$
\Delta d\left(\varphi_{0}\right)=\Delta d(z)=d_{0} \frac{z / z_{\alpha}}{1-z / z_{\alpha}} \cong d_{0} \frac{z}{z_{\alpha}}\left(1+\frac{z}{z_{\alpha}}+\cdots\right)
$$

It is also evident from the magnitude of $\mu d_{0}$ that the exponential can be approximated by equation (28):

$$
e^{-\mu(E) \Delta d\left(\varphi_{0}\right)} \cong[1-\mu(E) \Delta d(z)] \approx 1-\mu(E) d_{0} \frac{z}{z_{\alpha}}\left(1+\frac{z}{z_{\alpha}}\right)
$$

Therefore, the integral over energy spectrum became, equation (29):

$$
\int_{E} A_{0}(E) e^{-\mu(E) \Delta d(z)} \cong \int_{E} A_{0}(E)[1-\mu(E) \Delta d(z)] d E=A_{0} \equiv \rho(z) A_{0}
$$

Where $\langle\mu\rangle$ is the average of $\mu(E)$ over the spectrum $A_{0}(E)$ and $\rho(z)$ is well approximated by equation (30). 


$$
\rho(z) \cong 1-\langle\mu\rangle d_{0} \frac{z}{z_{\alpha}}\left(1+\frac{z}{z_{\alpha}}\right)
$$

Therefore, the equation (31) will be reduced to simpler form:

$$
f_{p}(z) \cong \rho(z) A_{0} \int_{s} \frac{1}{l} g\left(\frac{S}{l}\right) \Pi\left(\frac{z_{c}}{w}\right) d s \equiv \rho(z) \tilde{f}_{p}(z)
$$

Where $\tilde{f}_{p}(z)$ is defined as the primary beam axial dose profile with anode tilt, but without the heel effect. The approximations used to derive equation (31) are show in Table 1.

Table 1. Approximations used to derive the analytical function for primary dose profile.

\begin{tabular}{ccc}
\hline Description & Percentage $\%$ & Effect on dose profile \\
\hline $\begin{array}{c}\text { The same photon energy distribution. } \\
\text { Small angle 1-2 degrees. }\end{array}$ & $0.2 \%$ & Overestimate \\
$\begin{array}{c}\text { Small angle approximation, } \cos (\boldsymbol{\varphi})= \\
\mathbf{1}\end{array}$ & $\begin{array}{c}0.1 \% \text { to } 0.3 \% \text { for } \mathrm{T}=20 \mathrm{~mm} \text { to } 40 \\
\mathrm{~mm}, \text { respectively. }\end{array}$ & Overestimate \\
Disregarding $\left(1-\frac{s}{F} \cdot \cos \alpha\right)^{-2}$ & $\mathbf{0 . 8} \%$ & Over/Under estimation \\
Produce small differences at $\mathbf{z}= \pm \boldsymbol{a} / \mathbf{2}$ & $\leq \mathbf{0 . 2} \%$ & Overestimate \\
Center point approximation & &
\end{tabular}

The analytical equation used to validate the MC simulated primary profile is shown in equation (32), in which, the function $\rho(\mathrm{z}) \cong 1-\langle\mu\rangle d_{0} \frac{z}{z_{\alpha}}\left(1+\frac{z}{z_{\alpha}}\right)$ represents the anode selfattenuation (heel effect). For derivation details of $\rho(z)$ see Appendix A of (Dixon et al., 2005). This derivation allows to express $f_{p}(z)$ as.

$$
f_{p}(z)=\rho(z) A_{0}\left\{\frac{1}{2} \operatorname{erf}\left[\frac{\sqrt{\pi}}{c_{L}}\left(\frac{a}{2}+z\right)\right]+\frac{1}{2} \operatorname{erf}\left[\frac{\sqrt{\pi}}{c_{R}}\left(\frac{a}{2}-z\right)\right]\right\}
$$

The parameter $a$ is the projected collimator aperture onto the AOR, $\operatorname{erf}(u)$ is the error function, $c_{L}$ and $c_{R}$ represent the focal spot penumbra at $z= \pm a / 2$ and $A_{0}$ is the peak dose.

\subsubsection{Scatter Dose Profile}

Equation (33) and equation (34) show analytical expressions for the scatter profile component inside, $f_{s i}(z)|z| \leq a / 2$, and outside, $f_{s o}(z)|z| \geq a / 2$, of the primary beam, respectively. They were obtained from the convolution of the scatter Line Spread Function, $L S F=\eta \cdot l s f(z)$, (Boone, 2009) with the primary beam core function $A_{0} \Pi(z / a)$. Where, $l s f(z)$ is a unit area function (expressed in terms of its width $d$ as: $l s f(z)=d^{-1} h(z / d)$ ) and $\eta$ denotes the scatter-to-primary ratio. For more derivation details of the scatter dose profile, $f_{s}(z)=f_{s i}(z)+f_{s o}(z)$, see Dixon and Boone (Dixon and Boone, 2011). 


$$
\begin{aligned}
& f_{s i}(z)=A_{0} \eta\left[1-e^{-a / d} \cosh \left(\frac{2 z}{d}\right)\right], \quad|z| \leq a / 2 \\
& f_{\text {so }}(z)=A_{0} \eta \sinh \left(\frac{a}{d}\right) \exp (-2|z| / d), \quad|z| \geq a / 2
\end{aligned}
$$

\subsubsection{Total Dose Profile}

The total dose profile will be the sum between the primary and scatter profile. Therefore, the dose profile can be obtained as follow: $f(z)=f_{p}(z)+f_{s}(z)$. Where, Equation (32), Equation (33), and Equation (34) represent the primary and scatter components of the total profile.

\subsubsection{Helical Scanning Formulation}

For CT helical scan, the patient table is displaced along the $\mathrm{z}$-axis with constant velocity $v$. If $\dot{f}(z, t)$ is the instantaneous rate dose profile, the integral of $\dot{f}$ between the time interval $\left[-t_{0} / 2, t_{0} / 2\right]$ produce the cumulated dose profile $D(z)$, as shown in Equation (35).

$$
D(z)=\int_{-t_{0} / 2}^{t_{0} / 2} \dot{f}(z, t) d t
$$

Consider first the dose rate at center of the cylindrical phantom. In this particular case, the rate is constant because the X-ray beam is attenuated by the same thickness of phantom material (disregarding the attenuation of the patient table) and depends only on the translation motion along the $\mathrm{z}$-axis. Therefore, for a constant $\mathrm{x}$-ray fluence emission, the dose rate profile at the center can be redefined as $\dot{f}(z, t)=f(z-v t) / \tau$, where, $f(z)$ is the dose profile produced by single axial rotation in the static mode, $\tau$ is the time of one rotation and the function's argument was changed from $z$ to $z-v t$, to take into account the motion of the dose profile along the time. For convenience, $f(z)$ will be considered an even function (approximation, disregarding anode heel effect and a punctual focal spot). Therefore, Equation (36) is defined.

$$
D(z)=\frac{1}{\tau} \int_{-}^{t_{0} / 2} t_{0} / 2(z-v t) d t
$$

If, during the scan time $t_{0}$, the total irradiation length is $L$, with $L=v$. $t_{0}$. Equation (36) become Equation (37), with $y=v t$.

$$
D(z)=\frac{1}{v \tau} \int_{-L / 2}^{L / 2} f(z-y) d y
$$


From the last equation, the maximum dose occur at $\mathrm{z}=0$, and due to $f$ is an even function, Equation (37) become Equation (38).

$$
D(0)=\frac{1}{v \tau} \int_{-L / 2}^{L / 2} f(y) d y
$$

It is evident that the maximum dose $D(0)$ depends of the irradiation length L. Since $L$ determines the portion of the dose profile that is integrated to compute $D(0)$. For irradiation length, $L$, is sufficient to cover the scatter tail, then, the maximum dose $D(0)$ becomes the equilibrium dose $D_{e q}(0)$, as shown in Equation (39).

$$
D_{e q}(0)=\frac{1}{v \tau} \int_{-\infty}^{\infty} f(y) d y
$$

Equation (38) can be expressed in terms of a convolution product (Bracewell, 2000) between the dose profile $f(z)$ and a rectangular function $\Pi(y / L)$ which has an unit height and width, $L$, as shown in Equation (40). The equilibrium dose will be reached if the scatter tail of the dose profile is included into the box with length $L$ (Dixon, 2003)

$$
D(z)=\frac{1}{v \tau} \int_{-\infty}^{\infty} f(z-y) \cdot \Pi(y / L) d y=\frac{1}{v \tau} \cdot f(z) \otimes \Pi(z / L)
$$

For realistic dose profile $f(z)$, the cumulated $D(z)$ can be quite nonuniform, hence it will be useful to obtain the line integral of the dose along the z-axis which is called "dose line integral" and denoted DLI, similar that the "dose length product" (Dixon, 2003, AAPM, 2010).

$$
D L I=\int_{-\infty}^{\infty} D(z) d z=\frac{1}{v \tau} \cdot \int_{-\infty}^{\infty} \int_{-\infty}^{\infty} f(z-y) \Pi(y / L) d y d z
$$

Applying the Fourier method, the computation of Equation (41) becomes Equation (42). It is easy to see that $D L I$ can be computed from $D_{e q}(0)$.

$$
D L I=\frac{L}{v \tau} \int_{-\infty}^{\infty} f(z) d z=N \int_{-\infty}^{\infty} f(z) d z=L D_{e q}(0)
$$

Where $L$ is the scan length and $N$ is the total number of rotations. The product $v \tau$ is the table displacement per rotation. Therefore, from Equation (42) it can be seen that for faster table motion the $D L I$ is reduced and for slower table motions the $D L I$ increases.

A similar derivation is considered for positions off the axis of rotation. In this case, the dose rate profile is not constant over the X-ray source rotation mainly due to the non-uniform attenuation of the phantom material caused by the variation of the thickness of the phantom during a rotation, and also by the divergence of the x-ray beam. In the peripheral case, the 
instantaneous dose rate profile located at angle $\theta_{z}$ is periodic with period $\tau$ and can be represented as a function of the X-ray tube angle $\theta=\omega t(-\pi \leq \theta \leq \pi)$ (Dixon, 2003), as shown in Equation (43).

$$
\dot{f}(z, t)=\frac{1}{\tau} f\left(z, \theta-\theta_{z}\right)
$$

Therefore, the cumulated dose along the peripheral axis located at angle $\theta_{z}$ is represented by Equation (44).

$$
D\left(z, \theta_{z}\right)=\frac{1}{v \tau} \int_{-L / 2}^{L / 2} f\left(z-y, \theta-\theta_{z}\right) d y
$$

Adopting $y=v \tau$, the angular average dose is obtained by averaging the last equation over $\theta_{z}$, then Equation (45) is obtained.

$$
\left.\bar{D}_{a n g}(z)\right)=\frac{1}{v \tau} \int_{-L / 2}^{L / 2}\left\{\frac{1}{2 \pi} \int_{0}^{2 \pi} f\left(z-y, \theta-\theta_{z}\right) d \theta_{z}\right\} d y
$$

The average dose over $\theta_{z}$ (angular average dose), performed at a fixed value of $y$ and $\theta$, is the axial dose profile $f(x-y)$. Therefore, the angular average dose is represented by Equation (46) and in the convolution picture in Equation (47).

$$
\begin{aligned}
& \bar{D}_{\text {ang }}(z)=\frac{1}{v \tau} \int_{-L / 2}^{L / 2}\left\{\frac{1}{2 \pi} \int_{0}^{2 \pi} f\left(z-y, \theta-\theta_{z}\right) d \theta_{z}\right\} d y \\
& \bar{D}_{\text {ang }}(z)=\frac{1}{v \tau} \int_{-L / 2}^{L / 2} f(z-y) d y=\frac{1}{v \tau} f(z) \otimes \Pi(z / L)
\end{aligned}
$$

These equations have the same form as the helical dose distribution on the central axis. Therefore, all equations derived for the central axis apply equally well to the angular average dose, Equation (46) and Equation (47) (Dixon, 2003). This fact also ensures that dose equilibrium can be reached on the peripheral axis.

\subsection{Novel CT Dose descriptors}

As described in Section 2.2, the current $C T D I_{100}$ method quantifies the absorbed dose over the central region of the volume (of the CTDI phantom) irradiated by a CT examination of $100 \mathrm{~mm}$ of scanning length (ICRU, 2012, AAPM, 2008). For scanning lengths larger than $100 \mathrm{~mm}, C T D I_{100}$ underestimates the cumulated dose. Dixon states 'The widespread use of the $100 \mathrm{~mm}$ chamber seems to have been and ad hoc decision, and not supported by the physics' (Dixon, 2019). Actually, the origin of CTDI 100 low efficiency lies in the restriction of the integration limits of the integral in Equation (12) which was established between $50 \mathrm{~mm}$ to $+50 \mathrm{~mm}$ along the z-axis, Equation (13). An important evaluation of the CTDI 100 
efficiency was made by John Boone, who concluded that: "for $10 \mathrm{~mm}$ slice thickness, a $120 \mathrm{kVp}$ X-ray spectrum, and the PMMA head phantom, the efficiency of the CTDI 100 was $82 \%$ and $90 \%$ for the center and peripheral holes, respectively. The corresponding efficiency values for the body phantom were $63 \%$ and $88 \%$. These values are reduced by only $1 \%$ when a $40 \mathrm{~mm}$ slice thickness was studied, so the use of CTDI ${ }_{100}$ for $40 \mathrm{~mm}$ wide X-ray beam is no less valid than its use for $10 \mathrm{~mm}$ beam widths"(Boone, 2007). As concluded by John Boone, the efficiency of the $C T D I_{100}$ measurement even with $10 \mathrm{~mm}$ beam width is low, and consequently, CT dose metrics derived from $C T D I_{100}$ (as those described in section 2.2) may not be as accurate as desirable.

In order to adopt a more accurate methodology to assess the radiation output from a current CT examination, new dose descriptors are being proposed aiming to improve the evaluation of the dose performance in CT updating the current $C T D I_{100}$ methodology. In this scenario, dose descriptors such as: Dose Line Integral $(D L I)$, Equilibrium Dose $\left(D_{e q}\right)$, the product Equilibrium Dose x Pitch $\left(p x D_{e q}\right)$, the Approach to Equilibrium function $(H(L))$, and Equilibrium Scanning Length $\left(L_{e q}\right)$ represent more accurate alternatives and they are supported by AAPM and ICRU (AAPM, 2010, ICRU, 2012). Also, the Size Specific Dose Estimate $(S S D E)$ that take into account the patient size in the assessment of the patient dose will be commented despite it will not computed in this work.

\subsubsection{Dose Line Integral}

Dose line Integral, $D L I$, is the total integration of the cumulated dose profile, $D_{L}(z)$, computed along the central or peripheral axes of the CTDI phantom, as shown in Equation (41). It is proportional to the scan length $L$ in which the term of proportionality is the Equilibrium Dose, $D_{e q}$, (Dixon, 2003). Equation (48) and Equation (49) show the last statements.

$$
\begin{gathered}
D L I=\int_{-\infty}^{\infty} D_{L}(z) d z \\
D L I=L . D_{e q}
\end{gathered}
$$

\subsubsection{Equilibrium Dose}

Equilibrium Dose, $D_{e q}$, represents the asymptotic value of the cumulated dose profile, $D_{L}$, at $z=0$. The absorbed dose around the central point $(\mathrm{z}=0)$ of the accumulative dose profile increases as the scanning length, $L$, become more large, up to reach its equilibrium value, $D_{e q}$, 
as shown in Figure 27. Cumulated dose profiles presented in Figure 27.a and Figure 27.b were obtained from Monte Carlo simulation and tallied along the central and peripheral axes of the head CTDI phantom, for beam width, $\mathrm{T}=20 \mathrm{~mm}$, pitch=1.375, and X-ray spectrum of $120 \mathrm{kV}$.
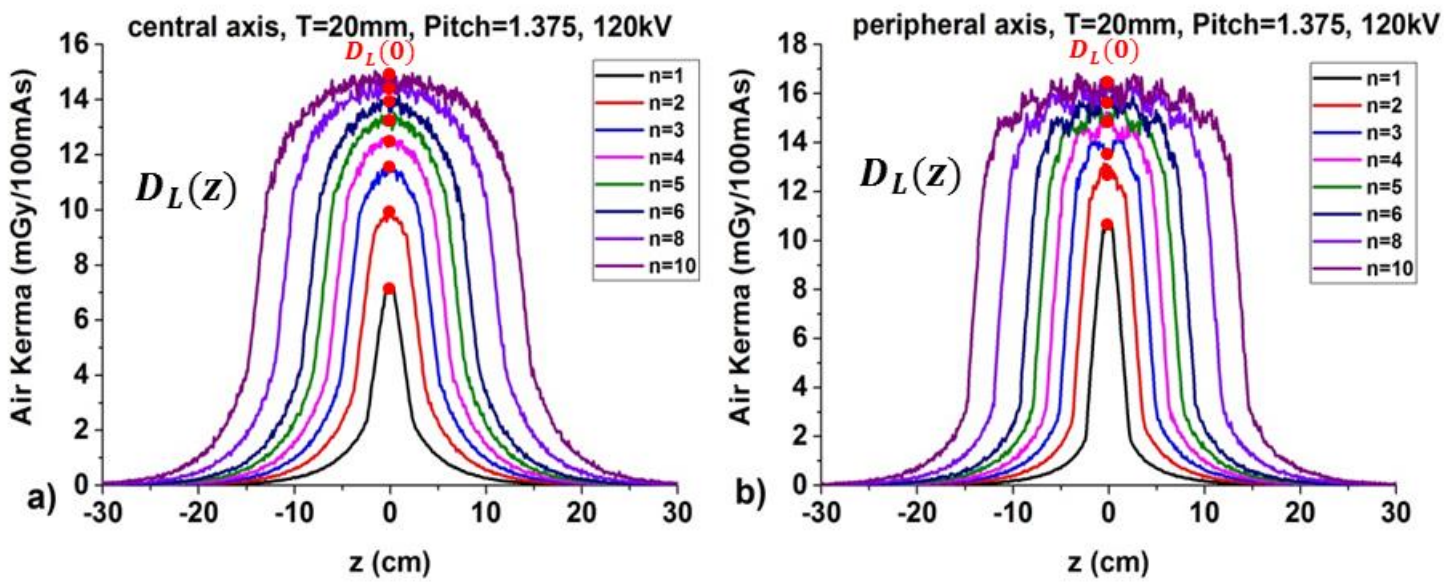

Figure 27. Cumulated dose profiles $D_{L}(z)$, from the MC modelling of the Helical CT scan mode for a number $n$ of $X$-ray source rotation. Along the a) central and b) peripheral axes of the head CTDI phantom.

\subsubsection{Approach to Equilibrium function}

The approach to equilibrium function (AAPM, 2010, Dixon and Boone, 2010) describes the fact that radiation dose from cumulative dose profile at $z=0$ at midpoint irradiated length $L$ increase with $L$ and asymptotically approaches the $D_{e q}$ for large $L$ (Dixon, 2003, Dixon and Ballard, 2007). Numerically, the approach to equilibrium function $H(L)$ is defined by the quotient expressed in Equation (50)

$$
H(L)=\frac{D_{L}(0)}{D_{e q}}
$$

Analytical expression for $H(L)$ is presented in Equation (51) where it is a result of the analytical integration of the primary and scatter components of the dose profiles (Equation (32), Equation (33), and Equation (34)) over the scan length, $L$.

$$
H(L)=\frac{1}{1+\eta}+\frac{\eta}{1+\eta}\left[1-e^{-L / d} \frac{\sinh (a / d)}{(a / d)}\right]
$$

Where $\eta$ denotes the scatter to primary ratio, and $d$ is the width of the line spread function as: $l s f(z)=d^{-1} h(z / d)$. Equation (51) can be reduced if beam width up to $40 \mathrm{~mm}$ are used (that is our case), in this case the term $\frac{\sinh (a / d)}{(a / d)}$ tends to 1 (Dixon and Boone, 2011). Then Equation (52) is presented, this equation is used as a basis to define parametric equations to fit the approach to equilibrium function obtained from the MC simulation of helical examination. 


$$
H(L)=\frac{1}{1+\eta}+\frac{\eta}{1+\eta}\left[1-e^{-L / d}\right]
$$

\subsubsection{Equilibrium Dose-Pitch product}

Since, the equilibrium dose $D_{e q}=D_{e q}(a, b)$ is proportional to the ratio $a / b$ and by definition is acquired at pitch $\mathrm{p}=\mathrm{b} / \mathrm{nT}, D_{e q}(a, b)=D_{e q}(a, p) \propto \frac{1}{p} \frac{a}{n T}$. Hence, the first alternative parametrization is the equilibrium dose-pitch product, shown in Equation (47). This equation is independent of $p$ (or $b$ ) and is identical to the equilibrium dose $D_{e q}(a, p)$ for a generalized pitch $p$ of unity. $a$, is the width ( $\mathrm{mm}$, along the axis of rotation) of the prepatient $\mathrm{z}$-axis collimator geometrically projected from the centroid of the X-ray source. The parameter $a$ correspond to the full-width at half of the primary-beam dose profile, $f_{p}(z)$. In the text, "collimation width" is interchangeably with $a$ and refers to a geometric projection on the AOR. $b$, is the scan interval (mm). In sequential scanning, $b$ is the midpoint-to-midpoint spacing between successive scans. In helical scanning, $b$ is the distance the tables moves continuously at constant velocity $v$ per rotation period $\tau: b=v \tau$, the table advance per rotation.

$$
\widehat{D}_{e q} \equiv p \cdot D_{e q}(a, p) \propto \frac{a}{n T}
$$

\subsubsection{Equilibrium Scanning Length}

$L_{e q}$, is the particular length $\mathrm{L}$ for which $\exp \left(-4 L / L_{e q}\right)=\exp (-4) \approx 0.0183$ i.e. for which the central $(\mathrm{z}=0)$ cumulative dose is within $2 \%$ of the limiting equilibrium value $D_{e q}$. In others words, $L_{e q}$ is a finite scanning length with respect to which scanning of a longer range makes for practical purposes a negligibly small difference in the estimation of $D_{e q}$.

\subsubsection{Size Specific Dose Estimate}

As mentioned before, the $C T D I_{100}$ method is defined to be measured in PMMA cylindrical phantoms of $16 \mathrm{~cm}$ or $32 \mathrm{~cm}$ diameter when head or body CT examination are considered, respectively. Due to the variety of patient sizes, the CTDI $I_{\text {vol }}$ (derived from $C T D I_{100}$ ) is not a good descriptor of the actual absorbed dose by the patient (McCollough et al., 2011). This fact has an important repercussion, especially in pediatric patients, in which the computation of absorbed dose based using the $C T D I_{v o l}$ displayed by the CT device, could lead to underestimating the patient dose by a factor of 2-3 if the $32 \mathrm{~cm}$ PMMA phantom was used for reference. Therefore, the Size Specific Dose Estimate, SSDE, was introduced in the 
AAPM report 204 (AAPM, 2011), updated in the AAPM report 220 (AAPM, 2014b), and ratified in the AAPM report 246 (AAPM-EFOMP, 2019). It takes into account the patient size in the assessment of a quantity proportional to the patient absorbed dose from a CT examination. In AAPM report 204, the computation of the SSDE is based on the displayed $C T D I_{v o l}$ of a CT device multiplied by a conversion factor as function of the patient effective diameter. The effective diameter can be understood as the diameter of the circle whose area is the same as that of the patient cross section, as shown in Figure 28. To determine the effective diameter, the patient cross section is approximated to an ellipse with its major and minor diameters are the lateral and anterior posterior (AP) dimensions of the patient cross-section, respectively. Then, the area of the ellipse is equal to the area of the circle with diameter equal to effective diameter. Equation (54) presents the relation, obtained after simple mathematical procedure, between the effective diameter with the AP and lateral patient dimensions.

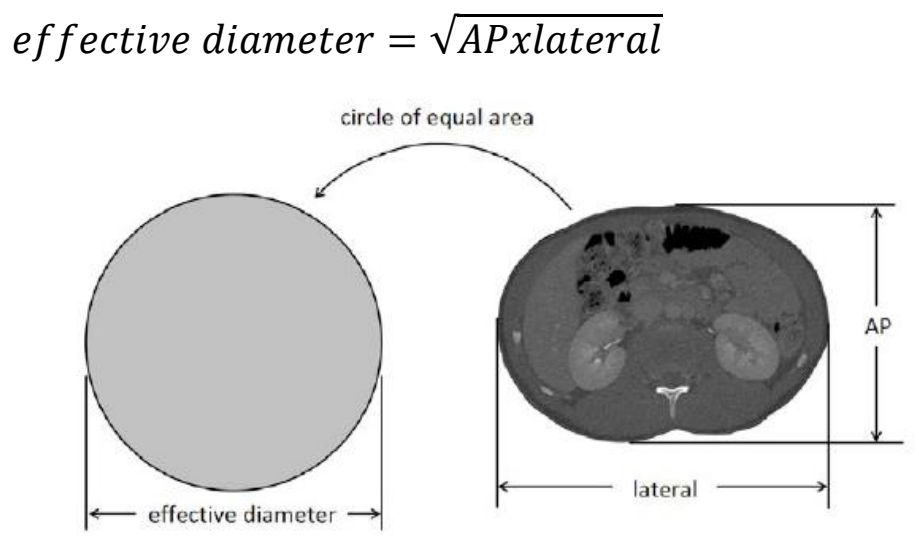

Figure 28. Graphical representation of the methodology used to determine the effective diameter from the anterior-posterior, AP, and lateral dimensions of the patient cross section. The effective diameter corresponds to a circle having an area equal to that of the patient cross-section on a CT image. Figures adapted from AAPM report 204(AAPM, 2011).

The lateral dimension can be obtained from a PA or AP CT radiograph, and the AP dimension can be determined by a lateral CT radiograph. The AAPM report 220 (AAPM, 2014b) revised the methodology to compute the correction factor as a function of the water equivalent diameter to take into account the X-ray attenuation of different parts of the human body with the same geometry, as thorax and abdomen. More recently, the AAPM report 246 (AAPM-EFOMP, 2019) suggests the use of the SSDE as a method to assess organ doses, and at the same time, adapt the methodology to take into account the tube current modulation, $T C M$. Equation (55) defines the SSDE as a function of the $z$ position to incorporate details of the patient for each reconstructed image. 


$$
\operatorname{SSDE}(z)=f_{W E D}(z) \cdot C T D I_{v o l}(z)
$$

Where, $C T D I_{v o l}(z)$ is the $C T D I_{v o l}$ value for each reconstructed image, $f_{W E D}(z)$ is the size-specific conversion factor from AAPM report 204 or 220. The mean SSDE over the entire scan range can be expressed as in Equation (56), where $\mathrm{N}$ is the total number of images.

$$
\overline{S S D E}=\frac{\sum_{Z=1}^{N} \operatorname{SSDE}(z)}{N}
$$

\subsection{Monte Carlo simulation of radiation transport}

The introduction of the integral-differential evolution equation for the velocity distribution of particles in the kinetic theory of gases by Ludwig Boltzmann, in 1872, had implications in the study of the radiation transport. That equation currently known as Boltzmann transport equation, which employs statistical principles to investigate the microscopic physical laws of electron-atom and photon-atom interactions, allows the computation of the radiation transport coefficients. Nevertheless, the analytics solution of the Boltzmann transport equation was not easy to do and was David Hilbert, in 1912, that got an approximate solution using an expansion in a potential series that is based on a parameter proportional to the mean free path of a gas. The solution of the Boltzmann transport equation has limited application in complex geometries, having more success in unlimited and semiinfinite media. Moreover, considering the geometric complexity of human organs and their great difference in absorption and scattering properties, the analytic approach becomes non practical (Zheng-Ming and Brahme, 1993). By the end of the 1950s, the computation power increased significantly and it allowed the implementation of numerical methods to understand the radiation transport in complex situations. The MC method was introduced by Nicholas Metropolis and Stan Ulam in 1949 (Metropolis and Ulam, 1949) and followed by the publications of Robert Wilson (Wilson, 1952) and Herman Kahn (Kahn, 1950a, Kahn, 1950b). Enrico Fermi had independently developed the method in Rome 15 years earlier studying moderation of neutrons but he didn't have a neat name for it. MC calculation represents an advantageous and alternative method to handle the radiation transport problem using a numerical solution of the Boltzmann equation in complex geometries.

Basically, MC methods allow to simulate the physical interaction of particles inside the matter and reproduce the energy loss after the interaction with the atoms or the production of secondary particles. The history of each particle and its evolution in terms of the energy 
transfer and the production of secondary particles receives the name of shower (a cascade of particles) of the particle. For instance, photon interactions are simulated over the elastic or inelastic collisions and the production of secondary particles, after each interaction a photon loss a fraction of its energy. Therefore, the shower is the evolution of the energy loss and, in contrast, an increment of the number of secondary particles. Because of the evolution of electron-photon shower has a random nature, MC method presents itself as an adequate option to deal with these problems. The use of the MC method to simulate the radiation transport for medical applications has been increasing in the recent years (Rogers, 2006, Andreo, 2018, Andreo, 2019).

The reliability of the physical interaction models and the tracking algorithms implemented in PENELOPE have been demonstrated by Sempau et al (Sempau et al., 2003) through an extensive set of benchmark comparisons of simulation results with experimental data. PENELOPE has been used for different applications in radiotherapy (Sempau et al., 2001, Feras et al., 2005) and dosimetry (Sempau and Andreo, 2006); it has also been benchmarked with other MC codes (Sung-Joon et al., 2004, Jocelyne et al., 2001, Siegbahn et al., 2003, Sechopoulos et al., 2015).

In order to model the Computed Tomography system required to achieve the main objectives of this work, the computational package PENELOPE/penEasy version 2015 was chosen (Sempau et al., 2011). It includes the code system PENELOPE (version 2014), which is an open source program, (PENetration and Energy Loss of Positrons and Electrons, photon simulation was introduced later) which is responsible to perform the Monte Carlo simulation of coupled electron-photon transport in complex geometries and arbitrary materials for a wide energy range, from a few hundred of $\mathrm{eV}$ to about $1 \mathrm{GeV}$. The package also includes the penEasy that is a steering main program which operates PENELOPE. This program manages the initial particle state (i.e., the radiation source) and the tallies for the quantities of interest, such as the absorbed dose in certain region of the space. Additionally, to construct the geometries, the software PENGEOM, compatible with PENELOPE/penEasy, is used to construct simple quadric geometries to establish the realistic scenario for the modelled system (Almansa et al., 2016).

PENELOPE is a Monte Carlo algorithm implemented in the computational code language Fortran and conceived to simulate the couple electron-photon transport in arbitrary materials. Overall, a detailed knowledge of radiation transport is required particularly in the design of radiation detectors (Titus, 1970, Berger and Seltzer, 1972), radiation dosimetry and 
radiotherapy (Andreo, 1991, Chetty et al., 2007). Because the random nature of the evolution of the shower (history of the particle), Monte Carlo calculation became a useful tool to deal with the radiation transport problem. The first MC simulation of photon transport was performed by Hayward and Hubbell in 1954 (Hayward and Hubbell, 1954), which followed 67 photons in a calculator.

When transport of photons is dealt, a detailed simulation must give the same results that the rigorous solution of the linear Boltzmann transport equation, apart to the uncertainties inherent to the method. In the case of simulation of electron transport, a detailed or condensed simulation could be performed, the last approach is used for soft electron collisions. In other words, because the very small energy loss of the electron in each interaction, the number of interactions increase dramatically if high energy electrons or thick geometries are part of the modelled system. Aiming to deal with this complex situation, the multiple-scattering theory is considered, which becomes feasible the simulation of the global effect of a large number of events in a track segment of a given length (step). This method was named as "condensed" Monte Carlo method (Berger, 1963). Noticeably, this method introduces systematic errors that become evident in cases when the result depend on the adopted step length (Bielajew and Rogers, 1986). Condensed schemes are not easy to deal, even when the results achieve a stabilization due to the reduction of the step length, it does not mean that the results are correct. For instance, in the case of the multiple-elastic-scattering theory of Moliere (Moliere, 1948), which is used in EGS4-based codes, the use of step lengths shorter than a few times the mean free path for elastic collisions produces a switch off in the process (Fernández-Varea et al., 1993). Condensed scheme also have difficulties in generating particle tracks in the vicinity of an interface, the condition is that the step length must be kept smaller than the minimum distance to the interface so as to make sure that the step is completely contained in the initial medium (Bielajew and Rogers, 1986).

In general terms, PENELOPE performs the simulation based on a scattering model that combines numerical databases with analytical cross section models for different interaction mechanisms and applicable for energies from a few hundred $\mathrm{eV}$ to approximately $1 \mathrm{GeV}$. The transport of electrons and positrons adopts a mix procedure which depends on the scattering angle $\theta$ or energy loss $W$ in each interaction. Hard interactions are simulated in detail when the scatter angle and energy loss are bigger than the preselected cut off values $\theta_{C}$ and $W_{C}$. On the other hand, soft interactions are simulated by means of multiple-scattering 
approaches when the scatter angle and energy loss are smaller than the corresponding cut off values of $\theta_{C}$ and $W_{C}$. 


\section{Chapter 3 - MATERIALS AND METHODS}

Section 3.1 describes the MC modelling of the radiation emission of the GE Discovery CT750HD with focus on the description on its main components, Section 3.2 presents the analytical relations between the novel dose descriptor in CT and Section 3.3 shows the experimental setup mounted to measure the single and cumulated dose profiles for helical examination.

\subsection{Monte Carlo Simulation}

Monte Carlo simulation of radiation transport in matter has become a useful tool in radiation dosimetry, medical imaging and radiological protection. Particularly, the simulation of coupled electrons-photons transport in tissue equivalent materials brought important advantages in medical diagnostic dosimetry due to the flexibility and ability to estimate quantities that are challenging to measure empirically. Therefore, in this work, the Monte Carlo code PENELOPE/penEasy (Salvat et al., 2015, Sempau and Andreo, 2006) was chosen to perform the simulation of coupled electron-photon transport in cylindrical simulators of PMMA to assess the deposited dose distribution in the phantom for photon energies ranging from a few keV's to $140 \mathrm{keV}$.

The MC simulation implemented in the present work was a two-stage process. The first one consisted in modelling the X-ray radiation emission of the CT system and the second one simulated the radiation transport in cylindrical phantom of PMMA, with the implementation of the X-ray source rotation and the phantom translation (helical scanning). The products of the first stage of the simulation were Phase Space Files (PSFs) which contain physical information of all particles that went through the pre-patient collimator. In the second simulation stage, the PSFs are used to model a helical scanning. Sections 3.1.1 and Section 3.1.2 describe in detail each stage and present the required information for reproducibility, as suggested by Sechopoulos et al. (Sechopoulos et al., 2015).

\subsubsection{MC modelling of the CT X-ray emission}

The CT systems GE Lightspeed Ultra CT and GE Discovery 750HD (General Electric Company, Boston, USA) were chosen as references to model the X-ray emission. Both systems have similar physical and geometrical characteristic summarized in Table 2 (Lewis et al., 2006), and both use the same GE Performix X-ray tube. 
Table 2. Technical specifications of the GE Discovery 750HD

and GE Lightspeed Ultra CT (Lewis et al., 2006)

\begin{tabular}{cc}
\hline & $\begin{array}{c}\text { Technical } \\
\text { specifications }\end{array}$ \\
\hline Focus-isocenter & 541 \\
distance, $F(\mathrm{~mm})$ & 162 \\
Focus-collimator & \\
distance, $F_{c}(\mathrm{~mm})$ & \\
X-ray tube & GE Performix \\
Focal spot size & \\
$(\mathrm{mm})$, quoted to IEC & $0.9 \mathrm{x} 0.9$ \\
$60336 / 2006$ standard & \\
Anode angle & $7^{\circ}$ \\
\hline
\end{tabular}

Most of the X-ray source models in diagnostic dosimetry consider that the X-ray beam is emitted from a point source disregarding the focal spot size, angle of the target surface, and target self-attenuation (Atherton and Huda, 1995, Boone, 2009, Kramer et al., 2017). In this work, the aforementioned characteristics had been implemented in the X-ray source model. Figure 29 shows the modelled components attempting to represent an actual CT system. The geometry was coded according to the PENGEOM format and syntax (Almansa et al., 2016). Figure 30 presents the setup used to compute the primary profiles from MC simulation.

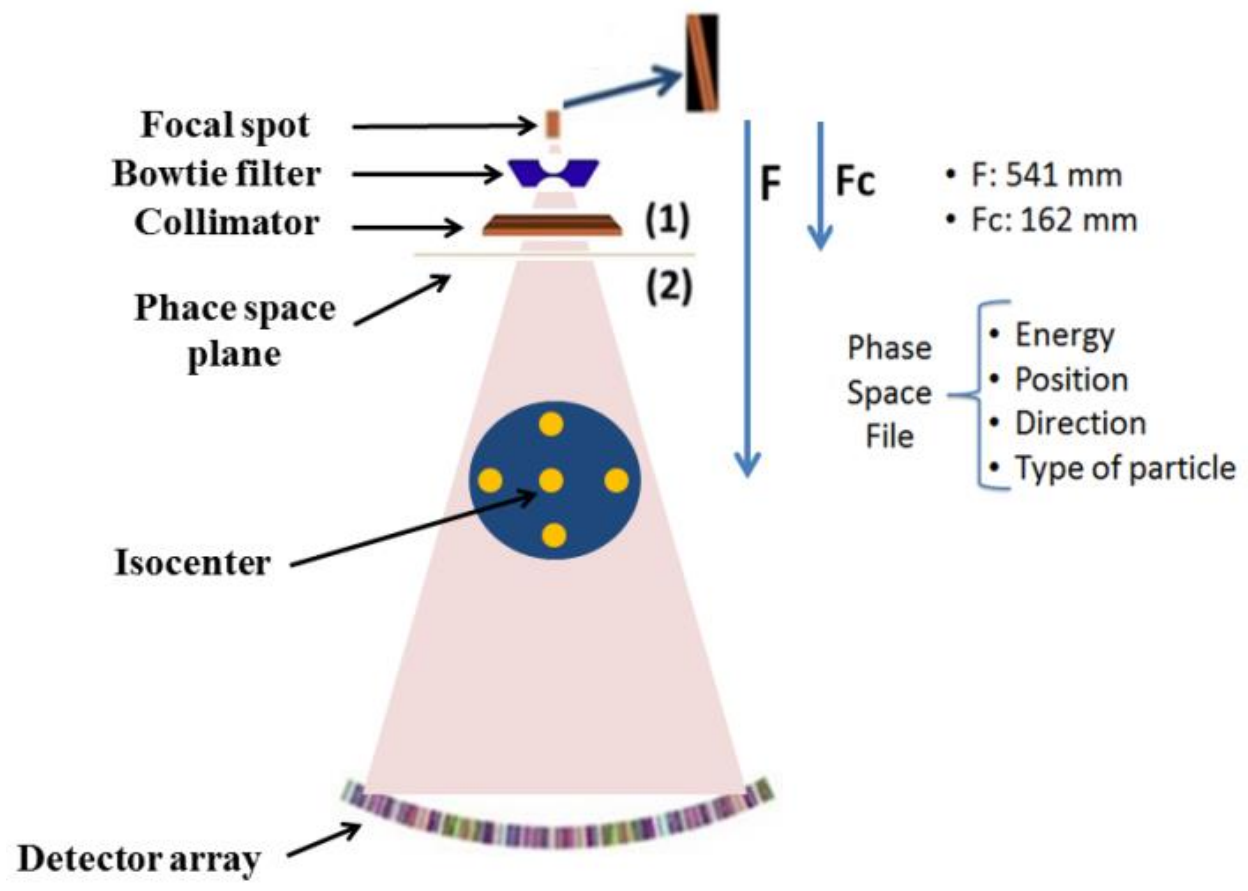

Figure 29. Schematic representation of a CT device, highlighting the x-ray source model used to assess the dose profile and the $\mathrm{CTDI}_{100}$ at center and peripheral positions. The orange line represents the position of the phase space plane, which serves to generate the phase space file. 


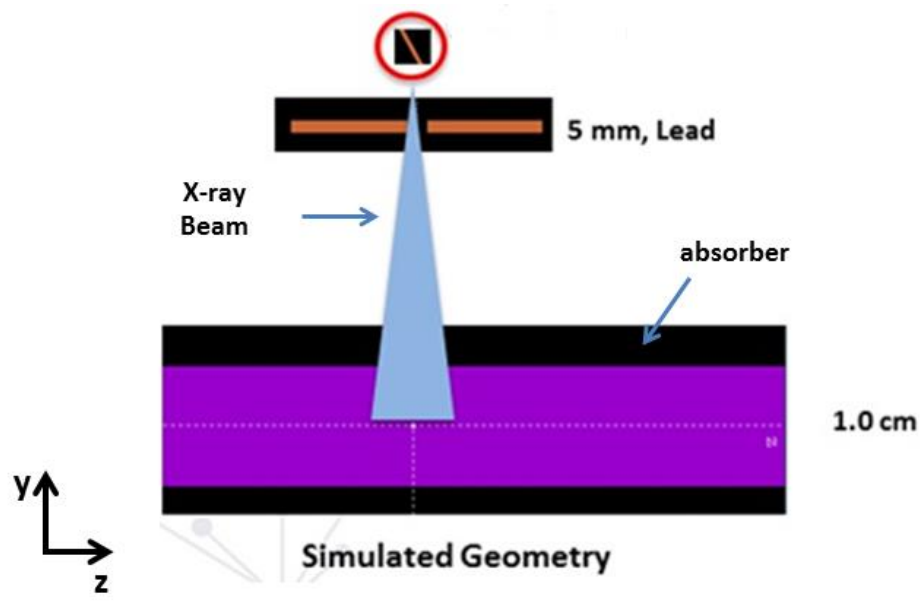

Figure 30. Setup used to compute by MC simulation the primary dose profiles. Note that in this case, there is not CTDI phantom, only a cylinder of $1 \mathrm{~cm}$ diameter was located along the AOR to absorb the X-rays.

In the discussion that follows, a brief description of each component of the modelled source is presented. Also, the procedures to generate the Phase Space Files are explained. . PSFs has been extensively used in radiotherapy research to implement helical examinations (Sterpin et al., 2008, Jeraj et al., 2004), for treatment planning in radiotherapy and dosimetry purposes (Brualla et al., 2009, Bush et al., 2007, Sempau et al., 2011, Cho et al., 2005). Also, IAEA offers a database of representative PSF's for external beam radiotherapy for accelerators and Co-60 units (https://www-nds.iaea.org/phsp/phsp.htmlx). Nevertheless, the use of PSFs to implement the radiation source model in computed tomography was not extensively addressed.

\section{- Focal spot:}

A one-dimensional focal spot (line) of $0.9 \mathrm{~mm}$ length was implemented as defined in the IEC 60336/2005 (IEC, 2005) standard. In order to include the anode self-attenuation in the source model, the one-dimensional focal spot was positioned at $3.4 \mathrm{~mm}$ beneath the surface of a tungsten block which is a typical target material in CT X-ray tubes. The choice of the location of the line source inside the tungsten block was based on comparison between the simulated primary profile and the analytical one trying to reproduce the effects of the target self-attenuation on the primary profile (see, Figure 52). To simulate the X-ray spectrum emitted by the X-ray tube, the semi-empirical TBC model (Tucker et al., 1991, Costa et al., 2007, Lopez Gonzales et al., 2015) was used to produce the photon energy spectrum emitted by a tungsten target at $80,100,120$ and $140 \mathrm{kV}$ with $6 \mathrm{mmAl}$ of additional filtration. As originally presented, the semi-empirical TBC model extends previous works to take into 
account the production of both bremsstrahlung and characteristic X-ray at varying depths within the target. Figure 31.a shows the X-ray spectra and Table 3 presents their physical characteristics. These spectra are in good agreement with those obtained by indirect measurement performed by Terini (Terini et al., 2017), as shown in Figure 31.b. The radiation emission distribution from the modelled line focal spot follows a Gaussian distribution with FWHM equal to $0.9 \mathrm{~mm}$, which is the optical length of the actual focal spot length (see, Figure 13).
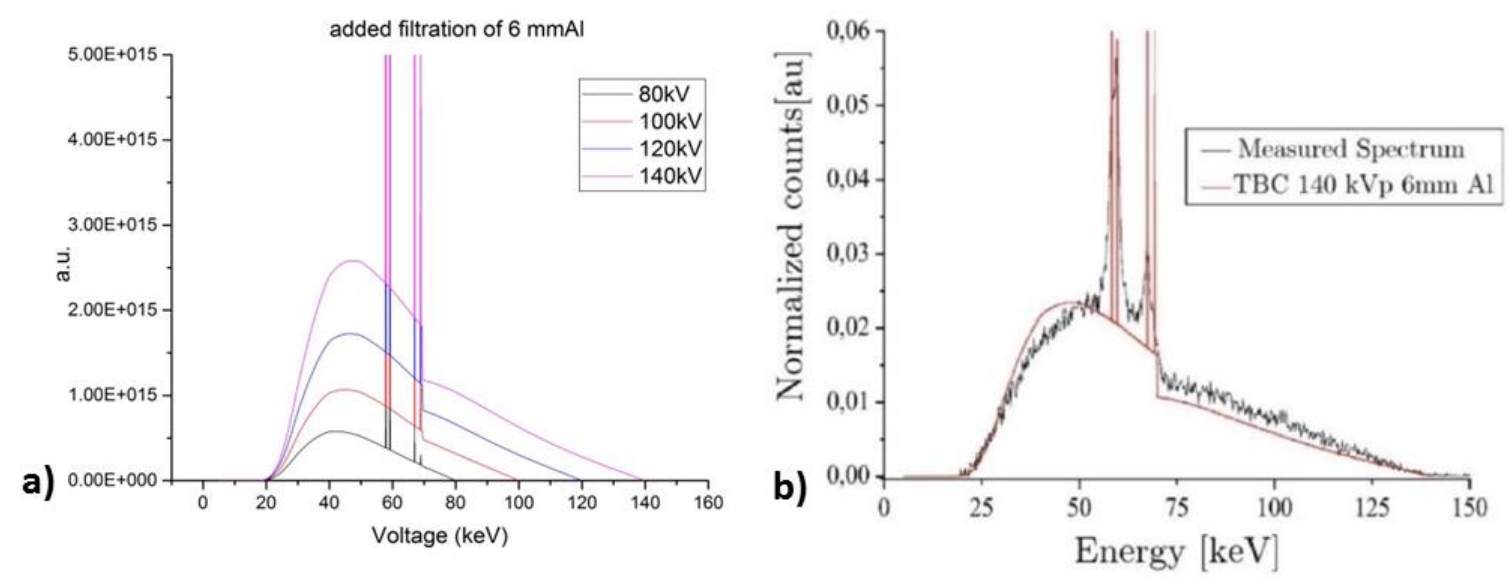

Figure 31. a) X-ray spectra generated by the TBC model used for the MC simulation, b) comparison between spectrum for $140 \mathrm{kV}$ and $6 \mathrm{~mm}$ Al with is corresponded measured one.

Table 3. Physical parameters about the TBC spectra for $80,100,120$, and $140 \mathrm{kV}$.

\begin{tabular}{cccccc}
\hline $\begin{array}{c}\text { Tube Voltage } \\
(\mathbf{k V})\end{array}$ & $\begin{array}{c}\text { Additional } \\
\text { Filtration } \\
(\mathbf{m m} \text { Al) }\end{array}$ & $\begin{array}{c}\text { HVL1 } \\
(\mathbf{m m} \text { Al) }\end{array}$ & $\begin{array}{c}\text { HVL2 } \\
(\mathbf{m m} \text { Al) }\end{array}$ & $\begin{array}{c}\text { Homogeneity } \\
\text { factor }\end{array}$ & $\begin{array}{c}\text { Effective } \\
\text { Energy } \\
\text { (keV) }\end{array}$ \\
\hline 80 & 6 & 5.45 & 6.54 & 0.83 & 47.63 \\
100 & 6 & 6.48 & 7.84 & 0.83 & 53.57 \\
120 & 6 & 7.19 & 8.69 & 0.83 & 58.16 \\
140 & 6 & 7.69 & 9.29 & 0.83 & 61.99 \\
\hline
\end{tabular}

\section{- Bowtie filter:}

Figure 32 illustrates the geometrical characteristics and dimensions of the modelled bowtie filter. It was constructed as being a truncated rectangular pyramid of Aluminium with two cylindrical sections removed. The bowtie filter imposes variable angular filtration along the fan angle (see, Figure 17). The filter seeks to homogenize the energy deposition of the detectors after imaging a phantom or a patient. As consequence, dose reduction is achieved (Mahesh, 2009, Kramer et al., 2017). As the characteristics of the bowtie filter in terms of its 
material or size are not known, dimensions presented in Figure 32 and aluminium material were assumed to model the filter.
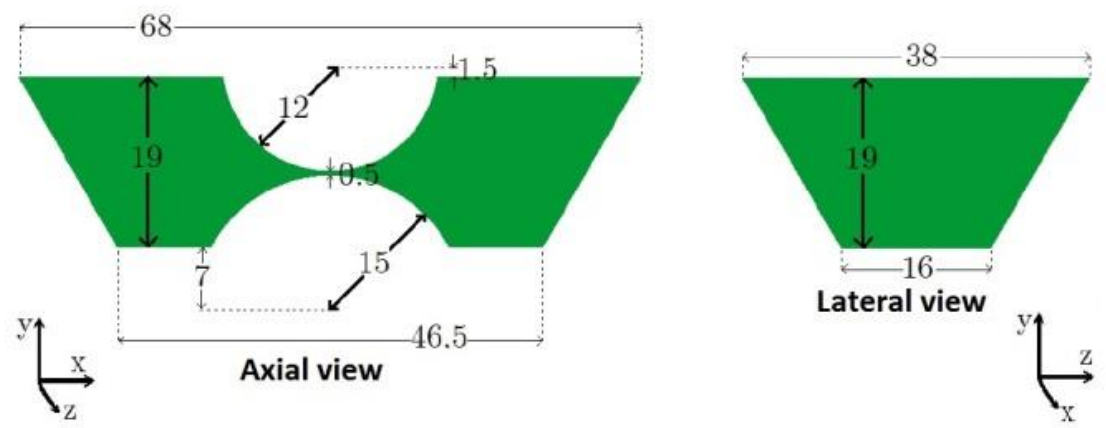

Figure 32. Geometrical characteristics and dimensions from Axial and Lateral view of the modelled bowtie filter. All numbers in the figure are in millimeters. Adapted from (thanks to João Terassi for AutoCAD design)

Another challenge related to including the filter in the simulation is its position between the focal spot and the pre-patient collimator. It is known that in Head and Body protocols the position of the bowtie filter is not the same or even there is a specific bowtie filter for each protocol. In order to solve this issue, two procedures were performed considering the homogenization of the deposited dose in the CT detectors and the deposited dose distribution inside the cylindrical CTDI simulator. The distance from the focal spot and collimator in the GE CT systems is $162 \mathrm{~mm}$ as indicated in Table 2. To find the best bowtie filter position for each specific case (head or body protocols) a set of 15 simulations were run changing the positions of the bowtie filter (between the focal spot and collimator) in steps of one centimeter, generating 15 PSFs considering $10^{9}$ initial histories. The change of the bowtie filter position to find adequate image quality parameters and dose optimization was evaluated in (Lück et al., 2014, Puerto, 2018). The last two mentioned approaches are described as follow:

First, the bowtie filter produces a compensation of the asymmetric attenuation of the $\mathrm{X}$-ray beam due to the patient. The objective is to homogenize the energy deposited in each detector element that impacts the quality in the reconstructed image and patient dose. Since, an accurate representation of the human characteristics in terms of attenuation is not possible for this work, the CTDI phantoms are taking to be our reference for the asymmetric beam attenuation. Therefore, for each one of the 15 PSFs and both head and body CTDI phantom of $16 \mathrm{~cm}$ and $32 \mathrm{~cm}$ diameter, respectively, the deposited energy in each detector element was assessed. The 15 PSFs were generated changing the distance between the focal spot and the bowtie filter in intervals of $1 \mathrm{~cm}$ ranging from $1 \mathrm{~cm}$ to $15 \mathrm{~cm}$. A graphical representation is 
shown in Figure 33 where the detector elements are covered by the phantom shadow. For all simulation, the deposited doses in each detector elements were tracked and compared between them. Finally, for the head and body phantom respectively, PSF was chosen that produced a more uniform dose in all detector elements.

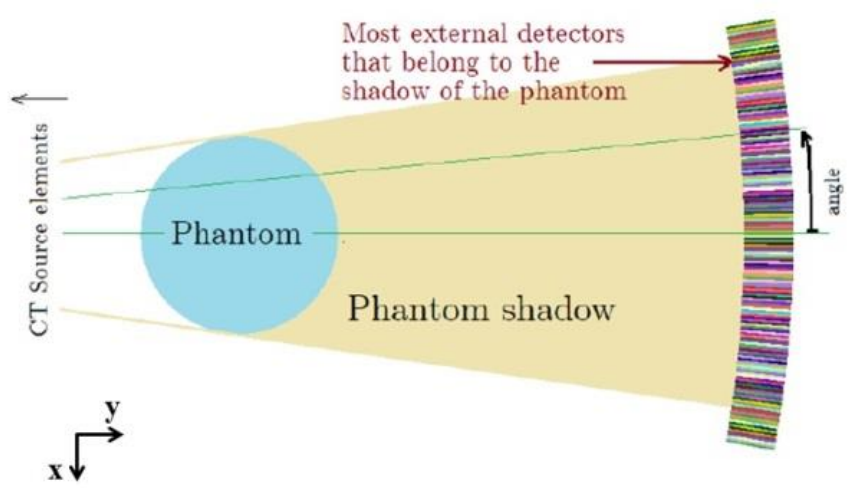

Figure 33. Schematic representation to find the best position of the bowtie filter that homogenize the deposited energy in all detector elements. The evaluation was performed for each head and body simulator. Adapted from (Puerto, 2018).

Second, Figure 34 shows the cylindrical CTDI simulator model used to evaluate the deposited energy on center and peripheral positions for head and body protocols. They are manufactured in PMMA with $1.19 \mathrm{~g} / \mathrm{cm}^{3}$ of mass density and $74 \mathrm{eV}$ of mean excitation energy. Figure 34.a shows five positions where the deposited dose were calculated, one at the center and four at $1 \mathrm{~cm}$ from the cylindrical surface, each of the 4 peripheral positions can be named as $0^{\circ}(\mathrm{NORTH}), 90^{\circ}(\mathrm{WEST}), 180^{\circ}$ (SOUTH) and $270^{\circ}(\mathrm{EAST})$. Figure $34 . \mathrm{b}$ illustrates 3 of the 5 cylindrical volumes with $10 \mathrm{~cm}$ length and $3 \mathrm{~cm}^{3}$ of volume. At this step, just the PSFs which produce the best homogenization in the detectors arrangements were chosen to perform the evaluation. After the simulation, the ratio of center to peripheral deposited energies was computed and compared with the experimental results.
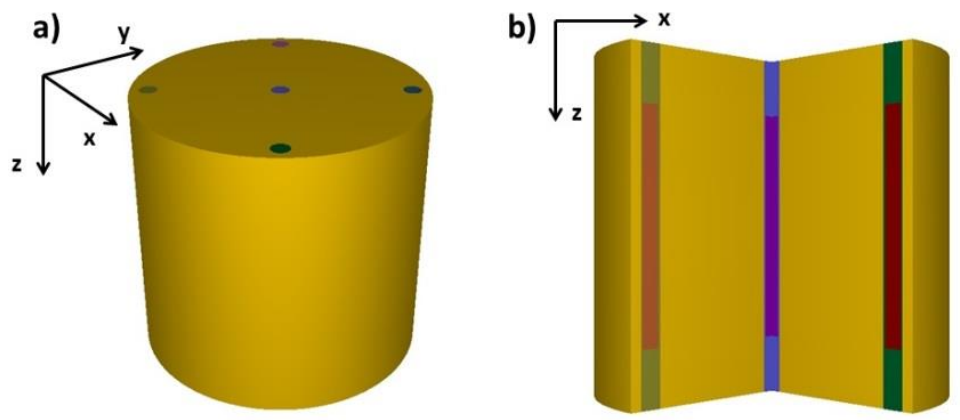

Figure 34. (a) cylindrical CTDI simulator used to compute the deposited energy at one center and four peripheral positions at $1 \mathrm{~cm}$ from the surface; (b) 3D view showing the cylindrical bodies with $10 \mathrm{~cm}$ of length and $3 \mathrm{~cm}^{3}$ of volume. 


\section{- Collimator:}

In order to produce a fan beam, two plates of lead were designed using PENGEON, to model the collimator used in CT systems. Each plate has $0.5 \mathrm{~cm}$ of width located at the same level at the desirable distance (or collimator aperture) which is sufficient to attenuate almost totally $140 \mathrm{keV}$ photons. The collimator was located at $162 \mathrm{~mm}$ from the focal spot as shown in Figure 29.

Summarizing, the first stage stores the information of all particles after the pre-patient collimator to create a PSF. This stage is named the static stage because the X-ray source was maintained static during the generation of the PSF. The use of PSFs to store the type of particle, energy, position, direction and statistical weight of all particles going through the pre-patient collimator makes the simulation more manageable, reducing the probability of errors and complexity with respect to the one stage simulation. Then, PSFs were used multiple times to compute the deposited dose distribution for single or helical CT examination.

\subsubsection{Simulation of single and helical CT scanning}

The second stage uses the PSFs to irradiate the cylindrical phantoms to compute the corresponding deposited dose distribution. At this stage, two subroutines PSFSource and PSFSinisrc corresponding to the module sourcePhaseSpaceFile.F of the PENELOPE/penEasy package were modified in order to implement the rotation of the PSF around the AOR and the translation along the z-axis to implement the CT helical scan mode. All particles stored in the PSF are evaluated and with the support of a random generator were positioned at 360 angle positions, or with a bin of $1^{\circ}$. At this stage, the single dose profile obtained from a single rotation of the PSF around the CTDI phantom was computed. To implement the helical scanning, instead of that the CTDI phantom is moving along the AOR, the z-coordinate of the particles is translated sampling uniformly the total scanning length and the same time the particle coordinate is rotated along the CTDI phantom.

The validation of the MC modelling of a CT examination was performed comparing the experimental $\mathrm{CTDI}_{100}$ at center and peripheral positions with the corresponding simulated ones. To derive CTDI values from MC simulation, the deposited dose in the five regions of the $3 \mathrm{~cm}^{3}$ were tallied. Experimental values of the CTDI are obtained with a pencil ion chamber which registers the air kerma inside its active volume. The MC simulation compute the deposited dose inside the cylindrical volumes of PMMA material. Therefore, a conversion 
factor may be applied to this value to be compared with the ion chamber reading. This procedure is needed to be able the comparison of our results with information from the literature such as: CTDosimetry datasheet (CTDosimetry, ImPACTScan) and simulated data from (Kramer et al., 2017). The simulations have been performed using $10^{7}$ particles and a cut off above $2 \mathrm{keV}$ for photons and $1 \mathrm{MeV}$ for electrons, in order to guarantee the absorption of the electron at the same position it was created. These considerations produce an estimation of the Kerma on the phantom material (Kramer, 2017). To assess the air kerma, the PMMA kerma has been multiplied by the ratio of the mass-energy absorption coefficients of the air and PMMA weighted by X-ray energy spectrum. Equation (57) shows the f-factor used to convert PMMA kerma to air kerma.

$$
f=\frac{\int_{E=0}^{E \max } \mathrm{E} \Phi(E)\left[\frac{\mu_{\text {en }}^{\text {air }}(E) / \rho^{\text {air }}}{\mu_{\text {en }}^{P M M A}(E) / \rho^{P M M A}}\right] d E}{\int_{E=0}^{E \max } \Phi(E) d E}
$$

Where $E$ is the photon energy, $\Phi(E)$ is the spectrum, and $\mu_{e n}^{x}(E)$ is the mass energy absorption coefficient ( $\mathrm{x}=$ air or PMMA). The above defined $f$-factor converts kerma in PMMA to kerma in air.

To record the deposited dose distribution inside the CTDI phantoms, three tallies incorporated in the PENELOPE/penEasy package were activated, they are: the Tally Cylindrical Dose Distribution, the Tally Spatial Dose Distribution, and the Tally Energy Deposition, as follow, a brief description of each tally is given:

\section{- Tally cylindrical dose distribution}

The absorbed dose per simulated history is tallied in the $r$ interval, $\left[r_{\min }, r_{\text {max }}\right]$, using $n$ bins, where $r$ stands for the radial distance $\sqrt{\left(x^{2}+y^{2}\right)}$. Corresponding intervals and bins are defined for the $\mathrm{z}$ axis. The resulting volume elements allow the scoring of a cylindrical dose distribution.

\section{- Tally spatial dose distribution}

The absorbed dose per simulated history is tallied in the $x$-interval $\left[x_{\min }, x_{\max }\right]$, using $n$ bins. Corresponding intervals and bins are defined for the $y$ and $z$ axis. The resulting bins allow the scoring of a 3D spatial dose distribution. 


\section{- Tally energy deposition}

The energy deposited per simulated history in each material is reported in an output file.

Another important remark for the simulations was the use of the computer servers available in the Group of Radiation Dosimetry and Medical Physics of the Department of Nuclear Physics of the Institute of Physics, University of São Paulo. The main features of the servers are presented in Table 4

Table 4. Main features of the servers available in the Group of Radiation Dosimetry and Medical Physics of the University oof São Paulo

\begin{tabular}{cccc}
\hline Name & Processor & Number CPUs & RAM \\
\hline WSDOSM & Intel(R) Xeon(R) CPU E5-2420 1.9GHz & 10 & $8 \mathrm{~GB}$ \\
WSDOSB & Intel(R) Xeon(R) CPU E3-1270 3.5GHz & 7 & $8 \mathrm{~GB}$
\end{tabular}

The number of photon histories used to create the PSF for each X-ray spectra were $10^{9}$ for both head and body helical protocols. In mean, the MC simulation time were, for the body phantom 16.5 hours and for the head phantom 8.6 hours.

\subsection{CT Dosimetry formulation}

The analytical formulation of the Dose Profiles for single and multiple (Helical or Axial mode) scans were adequately described in Section 2.3. Dose descriptors derived from the multiple scan dose profiles, such us: Dose Line Integral $(D L I)$, Equilibrium Dose $\left(D_{e q}\right)$, Pitch $x$ Equilibrium product $\left(p x D_{e q}\right)$, Approach to Equilibrium function $H(L)$, and Equilibrium Scanning Length $\left(L_{e q}\right)$ were defined formally in Section 2.4 (AAPM, 2010, ICRU, 2012, Dixon, 2019). In this Section the methodologies used to analyze the information derived from the simulated dose profiles are described, presenting formal relationships between the new dose descriptors, parametric models for fitting and a purpose for practical clinical use of the results of this work.

Equation (58) presents a useful analytical prediction by Dixon et al (Dixon, 2003), which states that the Dose Line Integral is equal to the product of the Equilibrium Dose and the Scanning Length $(L)$ for Helical/Axial protocols.

$$
D L I=L D_{e q}
$$

Where $L$ is computed by the product of the number of rotations $(N)$ and the table displacement in a single rotation $(b)$, or expressed in formula as: $L=n b$. As established in Equation (41), DLI can be obtained by total integration of the cumulative dose profile, $D_{L}(z)$, 
for Helical/Axial protocols. In this work, the DLI obtained along the central axis is denoted by $D L I_{c}$ and the $D L I$ obtained along the peripheral axis is denoted by $D L I_{p}$.

Also, aiming to obtain a representative value of the DLI for the whole CTDI phantom, the $D L I_{c}$ and $D L I_{p}$ were weighted by the coefficients $1 / 3$ and $2 / 3$, respectively, to obtain the weighted- $D L I$, denoted by $D L I_{w}$, as shown in Equation (59).

$$
D L I_{w}=\left(\frac{1}{3}\right) D L I_{c}+\left(\frac{2}{3}\right) D L I_{p}
$$

In this work, $D_{L}(z)$ were computed from MC simulation in head and body CTDI phantoms for the Helical scan. Figure 35.a and Figure 35.b each show a set of $D_{L}(z)$ tallied along the central and peripheral axes of a head CTDI phantom, respectively. Both sets of $D_{L}(z)$ were computed from MC simulations for Helical protocol with pitch value of 1.375, nominal beam width of $n T=20 \mathrm{~mm}$ and $120 \mathrm{kV} \mathrm{X-ray} \mathrm{spectrum.}$
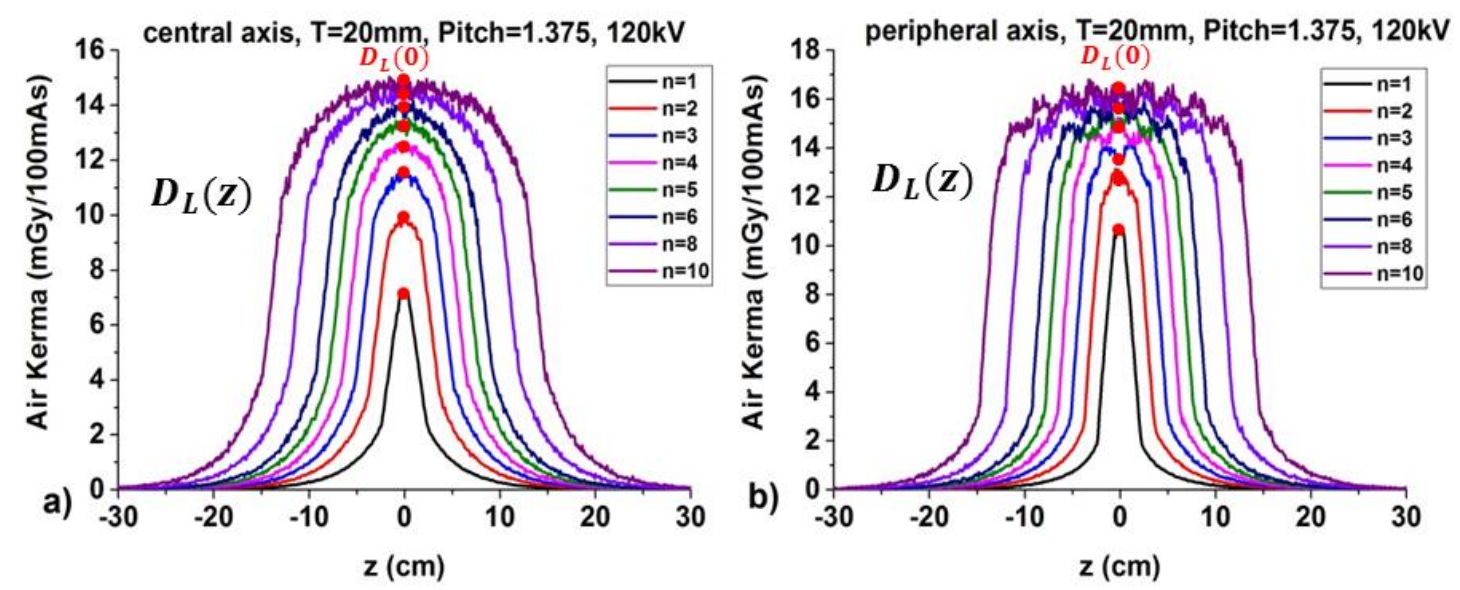

Figure 35. Cumulated dose profiles, $D_{L}(z)$, of CT Helical examinations for a number of X-ray source rotations, from 1 to 10 , along the a) central and b) peripheral axes of the head CTDI phantom. This data were obtained by Monte Carlo simulation with scan parameters, $\mathrm{nT}=20 \mathrm{~mm}$, pitch=1.375, and $120 \mathrm{kV} \mathrm{X-}$ ray spectrum.

As suggested by Equation (58), a graphical method using a plot of the DLI versus $L$ allows the assessment of $D_{e q}$ by fitting the plot with a linear model fixing the independent term to zero. The angular coefficient of the linear model should be an estimation of the $D_{e q}$ for a CT examination modelled by MC simulation. In this work, the $D_{e q}$ obtained from the set of $D_{L}(z)$ computed along the central axis is denoted by $D_{e q, c}$ and the $D_{e q}$ obtained from the set of $D_{L}(z)$ computed along the peripheral axis is denoted by $D_{e q, p}$. Also, aiming to obtain a representative value of the $D_{e q}$ for the whole CTDI phantom, the $D_{e q, c}$ and $D_{e q, p}$ were 
weighted by the coefficients $1 / 3$ and $2 / 3$, respectively, to obtain the weighted- $D_{e q}$, denoted by $D_{e q, w}$, as shown in Equation (60).

$$
D_{e q, w}=\left(\frac{1}{3}\right) D_{e q, c}+\left(\frac{2}{3}\right) D_{e q, p}
$$

Another way to obtain the $D_{e q}$ and others parameters that describe the dose distribution in the CTDI phantom is doing a plot of the dose value of $D_{L}(z)$ at $\mathrm{z}=0$. As mentioned above, $D_{L}(0)$ tends to an asymptotic value for a scan lengths sufficiently larger, this value is the $D_{e q}$. Equation (61) is the analytical formula that describe the plot of $D_{L}(0)$ versus $L$, this trend has been referred to as the rise-to-dose-equilibrium curve (Dixon and Boone, 2010). In Equation (62), in order to express explicitly the dependence with $L$, the function $h(L)$ has been defined as $h(L)=D_{L}(0)$, therefore, $D_{L}(0)$ and $h(L)$ are mathematically equivalent. If $D_{L}(0)$ is normalized to $D_{e q}$, it becomes the so-called approach to equilibrium function, $H(L)$, defined (Dixon and Boone, 2010), as:

$$
\begin{gathered}
D_{L}(0)=f_{p}(0) \frac{a}{b}\left\{1+\eta\left[1-e^{-L / d} \frac{d}{0} \sinh \left(\frac{a}{d}\right)\right]\right\} \\
H(L)=\frac{h(L)}{D_{e q}}=\frac{D_{L}(0)}{D_{e q}}
\end{gathered}
$$

$H(L)$ has an approximate form as a constant plus an exponential dependent term, as shown in Equation (52) leading to saturation, Equation (63) shows the approximatively form for $H(L)$.

$$
H(L)=1-\alpha \exp \left(-4 L / L_{e q}\right)
$$

Where $L_{e q}$ is the Equilibrium Scanning Length. This parametric model is used to fit the approach to equilibrium curve obtained from MC simulations.

\subsection{Experimental procedures}

The experimental measurements performed in the present work were conducted in order to validate the MC modelling of the $\mathrm{CT}$ radiation output which was described in Section 3.1.1. Radiation emissions from the GE Lightspeed Ultra CT and the GE Discovery CT750 HD (General Electric Company, Boston, USA) CT systems were chosen to be modelled. Since an objective of the present work was the assessment of the dose distribution in a CTDI phantom by $\mathrm{MC}$ simulation, so the $C T D I_{100}$ and the dose profiles descriptors were selected as indexes for the validation. There is consensus that they describe adequately the absorbed dose distribution in a CTDI phantom. Section 3.3.1 will describe the experimental methodology implemented to measure the $C T D I_{100}$, for specific cases of head and body 
protocols. The $C T D I_{100}$ descriptor is understood as an estimator of the CT radiation output, used for acceptance testing, quality control and also to compare acquisition protocols in different clinical services. Section 3.3.2 presents the experimental methodology adopted to measure the CT dose profile along the central and peripheral axes of a CTDI phantom. Dose profiles give a representative idea of the dose distribution along the central axis (or one parallel) of the CTDI phantom. Both, $C T D I_{100}$ and dose profiles measured for common clinical settings were compared with the MC results. The measurements were carried out in the Institute of Radiology of the School of Medicine of the University of São Paulo.

\subsubsection{Computed Tomography Dose Index, $C T D I_{100}$}

Despite its limitations in the assessment of the patient dose (McCollough et al., 2011), the $C T D I_{100}$ descriptor is a gold standard methodology well established and accepted by the community for evaluating dose properties of CT scanners and to compare CT procedures. The experimental method, reporting and management for the $C T D I_{100}$ are described in detail in the IAEA/TRS 457 and AAPM report $\mathrm{N}^{\circ} 96$ (IAEA, 2011, AAPM, 2008). Figure 36.a presents the ion chamber, radiation monitor, and the body and head CTDI phantoms used to measure the $C T D I_{100}$. These instrumentations are also described in the IAEA/TRS 457 and AAPM report $\mathrm{N}^{\circ}$ 96. Characteristics of the body and head CTDI phantoms are presented in Table 5. The height of the CTDI phantoms presented in Table 5 is $14.5 \mathrm{~cm}$, some manufacturers produce CTDI phantom with 14 or $15 \mathrm{~cm}$ of height as well. Figure $36 . \mathrm{b}$ illustrates the experimental setup employed to measure the $C T D I_{100}$ at central CTDI phantom position, which is named as $C T D I_{100, c}$. Special attention was given to the alignment in which the lasers of the CT unit were used for the correct positioning of the CTDI phantom and ion chamber. For the experimental determination of $C T D I_{100}$ in Figure 36.b, first, the head CTDI phantom (Nuclear Instruments Inc.) was placed on the CT table and aligned using the to match the coincidence between the central axis of the phantom and the z-axis of the CT coordinate system, which passes through the isocenter. The pencil ion chamber was introduced at the central hole and aligned using the lasers, matching the central indicators of the ion chamber with the intersection of the laser beams. Second, the irradiation was performed without CT table motion (axial scan technique) as indicated in the IAEA/TRS 457 (IAEA, 2011) and the AAPM report 96 (AAPM, 2008). After, for measurements of $C T D I_{100, p}$, the ion chamber position was changed to the corresponding peripheral positions. 
The procedures for measuring CTDI in the body phantom were the same as those used in the head phantom except for the phantoms substitution.

\section{CTDI phantoms}

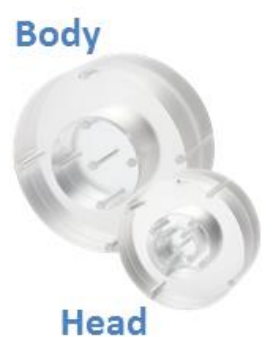

a)

\section{Ionization chamber and Monitor}

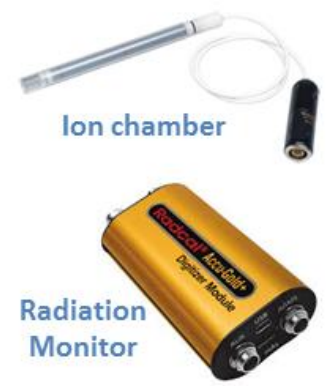

b)

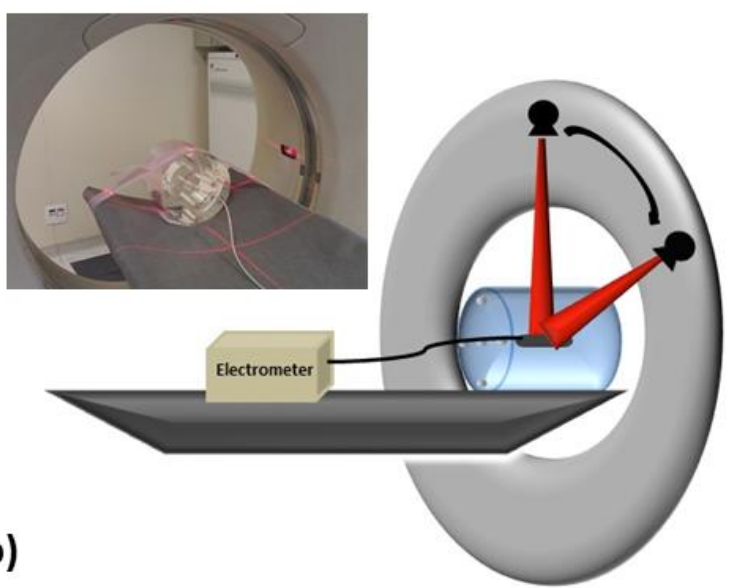

Figure 36. a) Body and head PMMA CTDI phantoms, pencil ion chamber, and radiation monitor. The CTDI phantoms have holes to introduce the pencil ion chamber. b) Schematic representation of the Experimental setup used to measure the $C T D I_{100}$ in CTDI phantoms, and actual arrangement is shown at top-left of the figure which represent the alignment procedure using the red lasers of the CT device.

Table 5. Specifications (complies with IEC 61223-3-5 and IEC 606001-2-44 standards) (https://www.sunnuclear.com/documents/datasheets/ctdi_phantoms.pdf)

\begin{tabular}{cc}
\hline Material: & Polymethyl-Methacrylate (PMMA/Acrylic) \\
\hline Density: & $1.19 \mathrm{~g} / \mathrm{cm}^{3}$ \\
\hline Alignment Makings: & Etched lines centered at the transverse, coronal and sagittal planes. \\
\hline Module: & Dimensions (OD x Length) \\
\hline Adult Body: & $32 \mathrm{~cm} \mathrm{x} 14.5 \mathrm{~cm}$ \\
\hline Adult Head/Pediatric Body & $16 \mathrm{~cm} \mathrm{x} 14.5 \mathrm{~cm}$ \\
\hline Pediatric Head: & $16 \mathrm{~cm} \mathrm{x} 14.5 \mathrm{~cm}$ \\
(Model 468-BHP only) & $19.9 \mathrm{~kg}$ \\
\hline Weight (total): & $1.31 \mathrm{~cm}$ \\
\hline Chamber Ports Diameter:
\end{tabular}

Additionally, measurements of the $C T D I_{100, \text { air }}$ free in air, named as $C T D I_{100, \text { air }}$, were performed aiming to be used as normalization factor for the $C T D I_{100, c}$ and $C T D I_{100, p}$, and to ponderate the $\mathrm{CT}$ radiation emission at the isocenter without the scatter medium (CTDI phantom or CT table). Figure 37 shows the experimental setup mounted for measurements of the $C T D I_{100, a i r}$. The pencil ion chamber with its polyacetal exterior cap (used as phantom adapter well as) is positioned along the CT AOR with its reference point matching the CT 
isocenter. The CT lasers were used to help in the ion chamber alignment and also the body CTDI phantom was used as support, as shown in Figure 37. Data of the CTDI $I_{100, \text { air }}$ is presented in Appendix A.

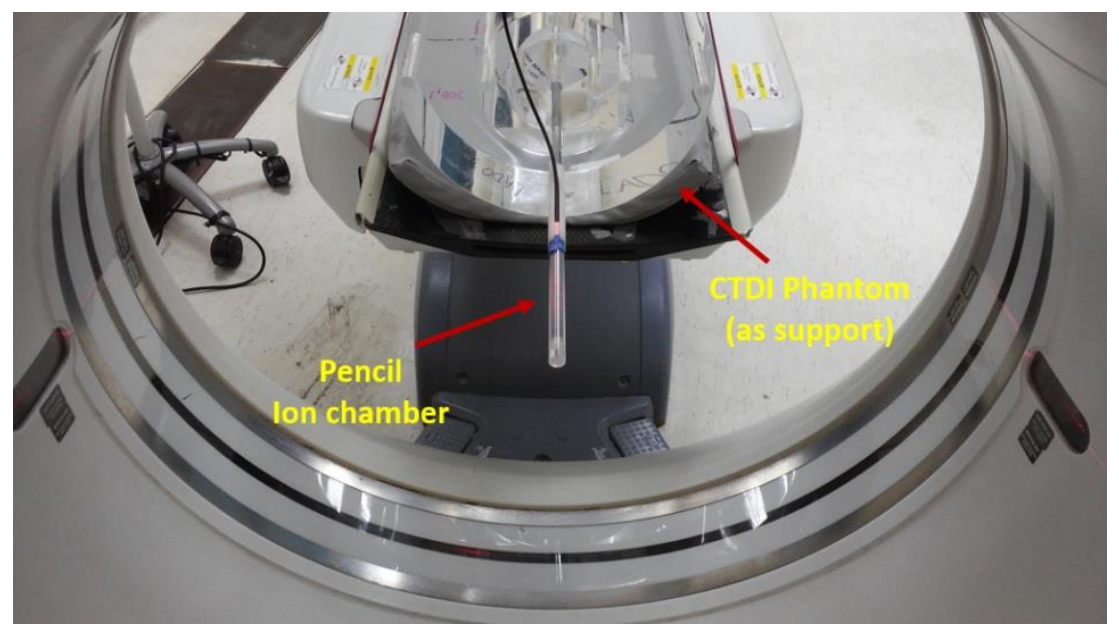

Figure 37. Experimental setup used to measure the $C T D I_{100, \text { air }}$ (free in air). In this case, the CTDI phantom is used as support.

The IAEA report 457 (IAEA, 2011) and the AAPM report 96 (AAPM, 2008) recommend the use of an ion chamber with $3 \mathrm{~cm}^{3}$ of active volume and $10 \mathrm{~cm}$ length (pencil ion chamber). In this work, two sets of $C T D I_{100}$ measurements were performed. The first one using the dosimetry system Radcal, model 10x5-3CT (S/N: 7986) with the electrometer Radcal model 9010 (S/N:90-2969) and the second one was done using the dosimetry system Radcal, model: 10x6-3CT (S/N: 05-1151) with the radiation monitor controlled model AGDM+ (S/N: 48-1054). Both measurement sets were calibrated in a traceable Secondary Standard Dosimetry Laboratory SSDL (IAEA, 2011). All experimental values of the CTDI 100 at the central and peripheral positions are reported in Appendix A. To analyze the uncertainty of the pencil ionization chamber used in this work, the uncertainty type B was considered following the technical specifications:

- 10x5-3CT: Calibration accuracy $\pm 4 \%$ (using X-rays, $150 \mathrm{kV}$ and $10.2 \mathrm{mmAl}$ HVL), Energy Dependence $\pm 5 \%$ ( $3 \mathrm{mmAl}$ to $20 \mathrm{mmAl} \mathrm{HVL}$ ), uniformity along length and partial volume exposure $\pm 5 \%$ ( to within $0.25 \mathrm{~cm}$ of chamber ends for a constant volume slice) The calibration factor was 1. Construction: C552 airequivalent walls and electrode; polyacetal exterior cap; $3 \mathrm{~cm}^{3}$ active volume; $2 \mathrm{~m}$, low-noise triax cable; $0.11 \mathrm{~kg}$. Therefore, the systematic uncertainty (Type B) is $8.1 \%$. 
- 10x6-3CT: Calibration accuracy $\pm 4 \%$ (using X-rays, $150 \mathrm{kV}$ and $10.2 \mathrm{mmAl}$ HVL), Energy Dependence $\pm 5 \%$ ( $3 \mathrm{mmAl}$ to $20 \mathrm{mmAl}$ HVL), uniformity along length and partial volume exposure $\pm 5 \%$ over central $95 \mathrm{~mm}$ of active length ( to within $0.25 \mathrm{~cm}$ of chamber ends for a constant volume slice) The calibration factor was 1. Construction: C552 air-equivalent walls and electrode; polyacetal exterior cap; $3 \mathrm{~cm}^{3}$ active volume; $1.5 \mathrm{~m}$, low-noise triax cable; $0.11 \mathrm{~kg}$. Therefore, the systematic uncertainty (Type B) is $8.1 \%$.

As the uncertainty type A was less than $0.07 \%$ for all $C T D I_{100}$ measurements, just the uncertainty type B was considered for comparisons with MC results and for calibration of the measured and simulated dose profiles.

The clinical protocols selected to be modelled were those that represent the most frequent CT examinations in a busy imaging facility located in São Paulo. Table 6 shows the clinical body and head protocols which represented around of $57 \%$ and $13 \%$ of the total CT procedures in 2016 at the Institute of Radiology of the School of Medicine of the University of São Paulo, respectively.

Table 6. Clinical body and head protocols chosen to be modelled by MC simulation.

\begin{tabular}{ccc} 
& \multicolumn{2}{c}{ Protocols } \\
\cline { 2 - 3 } Parameter & Body & Head \\
\hline Voltage (kV) & $80,100,120$, and 140 & $80,100,120$, and 140 \\
Rotation time (s) & 1.0 & 1.0 \\
Pitch & $0.516,0.984$, and 1.375 & $0.531,0.969$, and 1.375 \\
Bowtie filter & body & head \\
Collimation (mm) & 40 & 20 \\
Image number & 64 & 32 \\
Detector array & 0.625 & 0.625 \\
width (mm) & & \\
\hline
\end{tabular}

\subsubsection{Dose profiles}

Dose Profile measurements using OSL ribbon dosimeters inserted into the central or peripheral holes of the CTDI phantoms were performed. These OSL dosimeters are used for $\mathrm{X}$ and Gamma ray dosimetry and they are composed by $\mathrm{Al}_{2} \mathrm{O}_{3}: \mathrm{C}$ converted into powder deposited into long plastic tapes with $0.3 \mathrm{~mm}$ in thickness (Yukihara and McKeever, 2008). They were manufactured by the Landauer Luxel ${ }^{\mathrm{TM}}$ (Landauer, Inc., Glenwood, USA). The tapes were fractionated into ribbons of approximately $20 \mathrm{~cm}$ length as shown in Figure 38. The ribbons were covered by a black plastic to protect the dosimeters against natural radiation. 


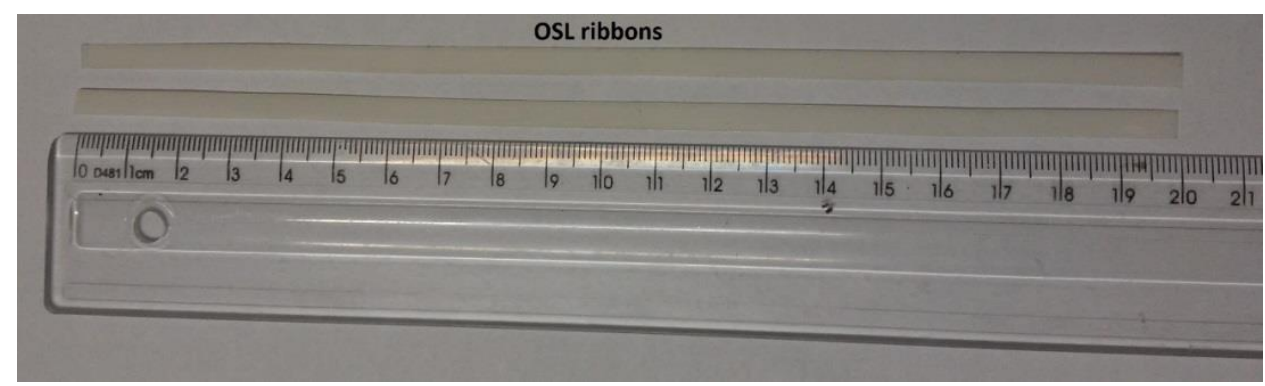

Figure 38. OSL Ribbon dosimeters used to measure the Dose Profiles in CTDI phantoms. They have $20 \mathrm{~cm}$ length approximately.

Figure 39 shows the experimental setup mounted to measure the primary and total dose profiles. Free-in-air measurement of a primary dose profile is performed without CTDI phantom. Primary profile reflects intensity and energy spectrum characteristics of the fan beam along the $\mathrm{CT} z$-axis. Analog to the ion chamber positioning to measure the CTDI 100 ,air, the OSL ribbon into a PMMA rod of roughly $1.0 \mathrm{~cm}$ diameter is aligned along the CT AOR and supported by a CTDI phantom as illustrated in Figure 39.a and Figure 39.b. The red lasers incorporated in the CT device were used for the correct alignment. The tube current and irradiation time for measurements of each primary profiles were $200 \mathrm{~mA}$ and $10 \mathrm{~s}$, respectively. To restrict the number of primary profiles measurements into the ones useful to validate the MC modelling, primary profiles for head bowtie filter with nominal beam width of $n T=20 \mathrm{~mm}$ and $n T=40 \mathrm{~mm}$, for body bowtie filter with nominal beam width of $n T=40 \mathrm{~mm}$ were measured, as suggested in Table 6. Figure 39.c shows the experimental arrangement mounted to measure the dose profiles in the body CTDI phantom. In this case, the CT table helps with the phantom's positioning, which is put and fixed with adhesive tape to the CT table. Five PMMA rods containing an OSL ribbon were introduced in the five holes of the body CTDI phantom, as illustrated in Figure 39.c. The tube current and irradiation time for measurements of each set of dose profiles were $200 \mathrm{~mA}$ and $20 \mathrm{~s}$, respectively. The procedures for measuring dose profiles in the head phantom were the same as those used in the body phantom except for the phantoms substitution. 


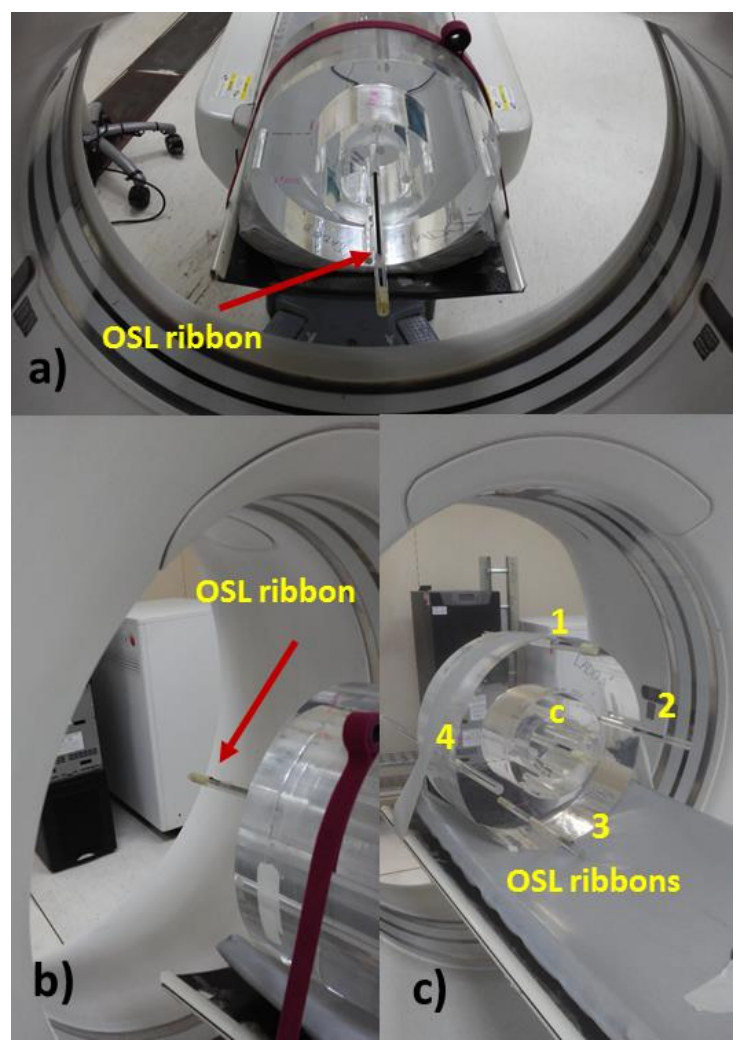

Figure 39. a) and b) experimental setup used to measure the primary dose profiles, and c) single dose profiles. They are measured using OSL ribbons.

Figure 40 shows the experimental setup used to measure the cumulated dose profiles for body helical/sequential protocols. The alignment was performed by centering the phantom in the middle of the gantry. Then, one OSL ribbon was put into the central hole and four in the peripheral positions. 


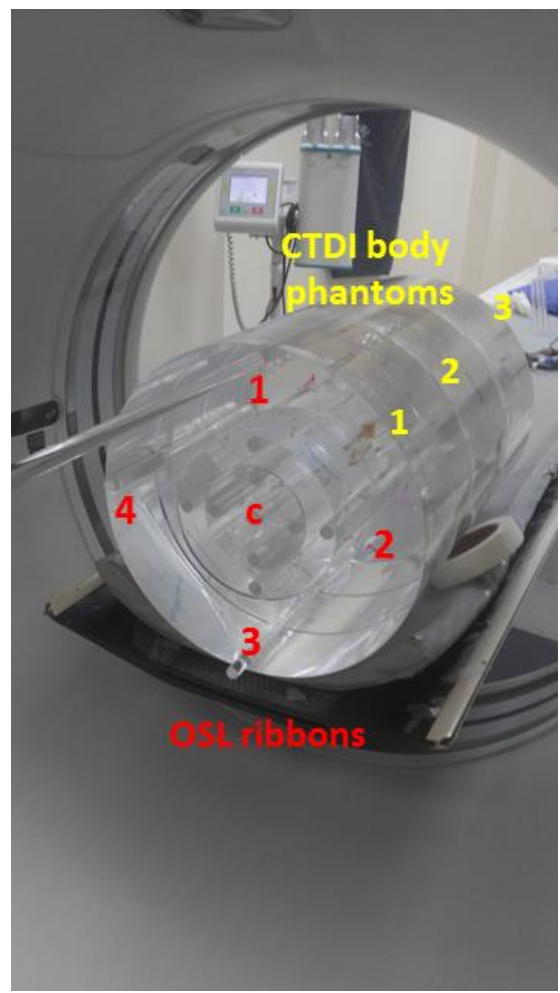

Figure 40. Experimental setup for the measurements of the cumulative dose profiles in three CTDI body phantoms.

After each set of expositions on the CT scanner, the irradiated OSL ribbons were lead to be read at the Group of Radiation Dosimetry and Medical Physics of the Institute of Physics, University of Sao Paulo. Figure 41.a shows the reading system that basically is composed by a photomultiplier tube (PMT) manufactured by the Sens Tech (Sens Tech Ltd., Surrey, UK), four green LEDs, and an Arduino control board (ARDUINO ${ }^{\circledR}$ ). The OSL ribbon is fixed on the support which is pushed by a stepper motor to pass across the reading system. The method of the reading process is based on the Pulsed Optically Stimulated Luminescence (POSL) in which the intermittent green LED light stimulates the OSL ribbons that emit light which reach the PMT. Figure 41.b shows an schematic representation of the reading system in which is possible to identify the optical filters GG495 that eliminate emissions of low wavelength from the LEDs. In the same figure, the filter Corning 5-58 is indicated which protect the PMT of the stimulation light. The Arduino control board does the interface with the computer with a control program written in LabView ${ }^{\mathrm{TM}}$ and $\mathrm{C}$ language (Umisedo and Yoshimura, 2017) 

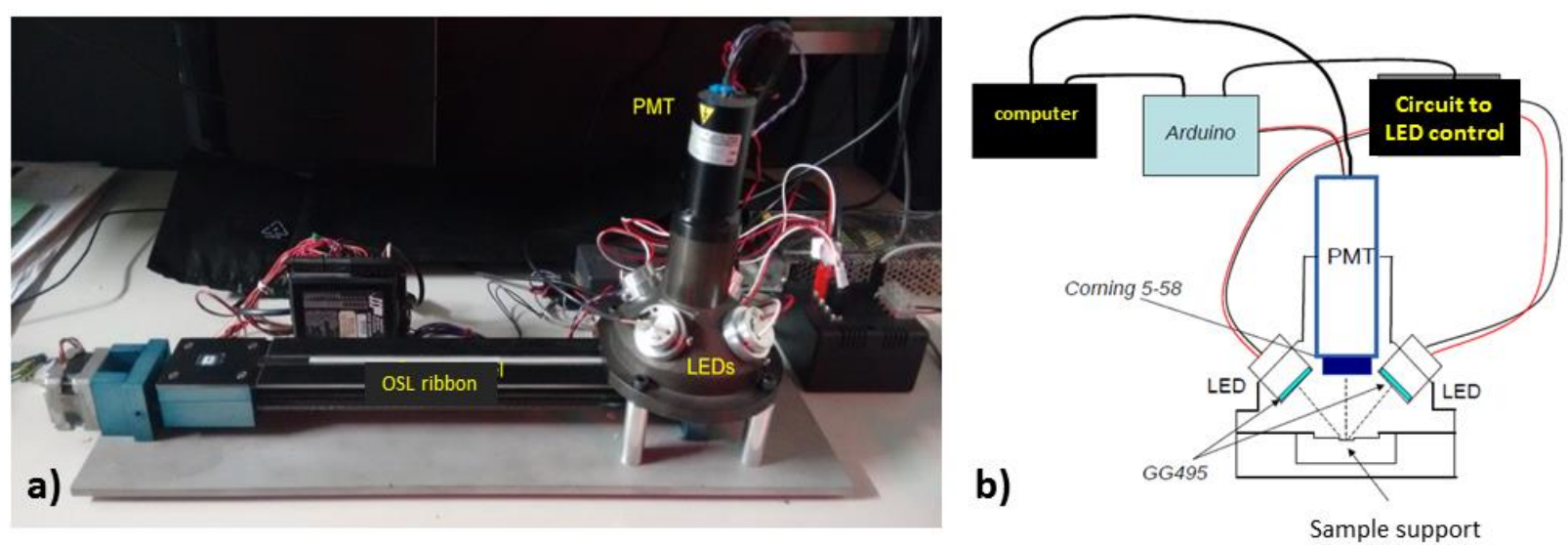

Figure 41. a) Reading system developed to register unidimensional dose profiles. It is based on the Pulsed Optically Stimulated Luminescence (POSL). b) Schematic representation of the reading system which uses LED to stimulate the OSL ribbon. The system is managed by an Arduino control board. Figures adapted from (Umisedo and Yoshimura, 2017)

Around 80 ribbons were employed in this work that as mentioned before they were used in two sessions of programmed measurements at the Institute of Radiology of the School of Medicine of the University of São Paulo. After the first irradiation and reading, each of the 80 ribbons were exposed to white fluorescent light emitted from a lamp during roughly 12 hours. The spatial resolution of the measured dose profiles is determined by the configuration of the POSL reader that was set to $1 \mathrm{~mm}$ per second. The LED stimulation is $4.6 \mathrm{~ms}$ and the interval between each stimulation is $13.5 \mathrm{~ms}$.

The signal registered by the POSL reader along the length of the OSL ribbons is proportional to the $\mathrm{X}$-ray fluence that reach the corresponding ribbons at the central and peripheral axes of the CTDI phantom. Also, this kind of OSL dosimeter $\left(\mathrm{Al}_{2} \mathrm{O}_{3}: \mathrm{C}\right)$ has a linear response with the radiation exposure that was subdued. The linearity and energy response for the OSL dosimeters used in this work was analyzed by Giansante et al. (Giansante et al., 2018). Figure 42 shows the calibration procedure of the simulated dose profiles. The calibration was performed using the corresponding $C T D I_{100}$ value for the central or peripheral axes of the CTDI phantom. As the ion chamber integrate the air kerma along of its $10 \mathrm{~cm}$ length, after the calibration the sum of the $10 \mathrm{~cm}$ central region of the simulated dose profiles should have the same value of their corresponding $C T D I_{100}$. 
Head

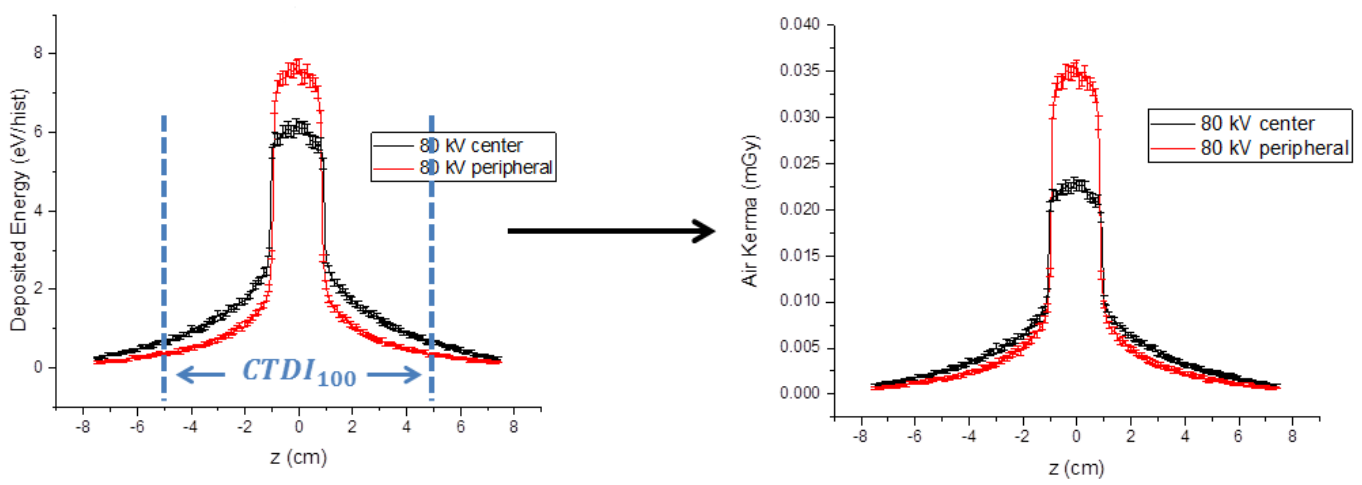

Figure 42. Calibration procedure of the simulated dose profiles, the corresponding $C T D I_{100}$ values are used for the calibration. As the measurement of the $C T D I_{100}$ is performed with a ion chamber of $10 \mathrm{~cm}$ length, the $10 \mathrm{~cm}$ central region of the dose profile was factored to its integral result in the $C T D I_{100}$ value. 


\section{Chapter 4 - RESULTS AND DISCUSSIONS}

This chapter begins with Section 4.1, which describes the validation of an X-ray source model based on a MC method and constructed for CT dosimetry purposes. The validation was sequentially conducted considering two approaches. It began comparing the values of $C T D I_{100}$ ratios obtained from MC simulations and experimental measurements, followed by correlating the dose profile distributions obtained from MC simulations with those from experimental measurements and computed from analytical formulas. Section 4.1.2.1 presents the MC modelling of a helical CT examination performed on head and body CTDI phantoms with fixed technical parameters. The cumulated dose profiles from MC simulations for the helical mode are also illustrated and discussed. Section 4.3 shows the dosimetry quantities: Equilibrium Dose, $D_{e q}$, Dose Line Integral, $D L I$, and Equilibrium Dose $\mathrm{x}$ pitch product computed from the cumulated dose profiles, which were calibrated using these dosimetric units (mGy/100 mAs). The Equilibrium Scanning Length, $L_{e q}$, are also presented as additional results. Additionally, comments about the efficiency of the $C T D I_{100}$ method to estimate patient dose are presented. This section also presents results for simulation on CTDI phantoms of polyethylene, water and a water equivalent material.

\subsection{Validation of the X-ray source model}

Two criteria were chosen to verify the MC modelling capability to represent the X-ray emission of the GE Discovery CT750 HD scanner (General Electric Company, Boston, USA). The first criterion chooses the $C T D I_{100}$ metric to verify the $\mathrm{MC}$ simulation of the deposited dose in $3 \mathrm{~cm}^{3}$ at one central and four peripheral positions of the CTDI phantom. $3 \mathrm{~cm}^{3}$ is the active volume of the pencil ion chamber used for $C T D I_{100}$ experimental measurements. The $C T D I_{100}$ was chosen since it is used for CT dosimetry evaluation (IAEA, 2011), and it is important that our model provides this quantity accurately. $C T D I_{100}$ does not offer details of the dose distribution inside the phantom, it only integrates the radiation exposure in the ion chamber active volume. Therefore, aiming to evaluate the dose distribution in the CTDI phantoms, the second approach compares the dose profiles computed by MC simulation along the central and peripheral phantom axes with the corresponding dose profiles obtained from experimental measurements and analytics formulas. 


\subsubsection{Validation based on $C T D I_{100}$ ratios}

Table 7 shows experimental values for $C T D I_{100, c}$, and $C T D I_{100, p}$ measured in head and body CTDI phantoms, $C T D I_{100, w}$, computed from $C T D I_{100, c}$ and $C T D I_{100 . p}$ according to Equation (14), and $C T D I_{100, \text { air }}$ measured free-in-air for the head and body bowtie filters. The data in Table 7 corresponds to nominal beam widths of $n T=10 \mathrm{~mm}$, and $n T=20 \mathrm{~mm}$ for the head phantom and $n T=10 \mathrm{~mm}$, and $n T=40 \mathrm{~mm}$ for the body phantom. All $C T D I_{100}$ values are normalized by the product between the X-ray tube current $(100 \mathrm{~mA})$ and irradiation time (1 s). For all measurements the large focal spot was selected, and the tube potentials were 80, 100,120 , and $140 \mathrm{kV}$. The systematic uncertainty (Type B) for the CTDI $I_{100}$ values was estimated as $8.1 \%$, as described in Section 3.3.1.

Table 7. Measured $C T D I_{100, a i r}, C T D I_{100, c}$ and $C T D I_{100, p}$ and computed $C T D I_{w}$ (according to Equation (14)) for head and body CTDI phantoms and for tube potentials of 80, 100, 120 and $140 \mathrm{kV}$. Nominal beam width, of $n T=10$, and $n T=20 \mathrm{~mm}$ for head phantom and $\mathrm{nT}=10$, and $\mathrm{nT}=40 \mathrm{~mm}$ for body phantom. For all measurements the systematic uncertainty (Type B) was 8.1\%, and the large focal spot was selected. Data for the GE Discovery CT750 HD scanner (General Electric Company, Boston, USA).

\begin{tabular}{|c|c|c|c|c|c|c|}
\hline \multicolumn{2}{|c|}{$(\mathbf{m m})$} & \multirow{2}{*}{$\frac{(\mathrm{kV})}{\text { Voltage }}$} & \multicolumn{4}{|c|}{ (mGy/100mAs) } \\
\hline & $\mathbf{n T}$ & & CTDI 100, air & $C T D I_{100, c}$ & $C T D I_{100, p}$ & $C T D I_{w}$ \\
\hline \multirow{8}{*}{ Head } & \multirow{4}{*}{$10 \mathrm{~mm}$} & 80 & 18.1 & 9.3 & 10.0 & 9.8 \\
\hline & & 100 & 30.1 & 17.5 & 18.1 & 17.9 \\
\hline & & 120 & 44.0 & 27.4 & 27.7 & 27.6 \\
\hline & & 140 & 59.8 & 38.8 & 38.6 & 38.7 \\
\hline & \multirow{4}{*}{$20 \mathrm{~mm}$} & 80 & 11.2 & 5.6 & 7.5 & 6.8 \\
\hline & & 100 & 18.7 & 10.6 & 13.1 & 12.3 \\
\hline & & 120 & 27.6 & 16.6 & 19.8 & 18.7 \\
\hline & & 140 & 37.8 & 23.6 & 27.6 & 26.3 \\
\hline \multirow{8}{*}{ Body } & \multirow{4}{*}{$10 \mathrm{~mm}$} & 80 & 13.0 & 2.0 & 5.7 & 4.5 \\
\hline & & 100 & 23.1 & 4.7 & 10.7 & 8.7 \\
\hline & & 120 & 35.5 & 8.0 & 17.1 & 14.0 \\
\hline & & 140 & 49.9 & 12.1 & 24.5 & 20.3 \\
\hline & \multirow{4}{*}{$40 \mathrm{~mm}$} & 80 & 7.6 & 1.2 & 3.4 & 2.7 \\
\hline & & 100 & 13.8 & 2.7 & 6.6 & 5.3 \\
\hline & & 120 & 21.2 & 3.9 & 10.1 & 8.0 \\
\hline & & 140 & 30.0 & 7.0 & 14.4 & 12.0 \\
\hline
\end{tabular}

As mentioned before, the validation of the X-ray source model was conducted comparing the ratios $C T D I_{100, c} / C T D I_{100, p}$ and $C T D I_{w} / C T D I_{100, a i r}$ between simulated and measured data from the GE Discovery CT750 HD scanner (General Electric Company, Boston, USA). Additional comparison was added in this work including data of the CTDosimetry ${ }^{\mathrm{r}}$ spreadsheet for the GE Lightspeed Ultra (General Electric Company, Boston, USA). This comparison could be performed since both GE Discovery CT750 HD and GE 
Ligthspeed Ultra have the same X-ray tube model and geometry, therefore, they have compatible X-ray emissions. Table 8 and Table 9 present the ratios $C T D I_{100, c} / C T D I_{100, p}$ and $C T D I_{w} / C T D I_{100, a i r}$ for simulated, measured and CT Dosimetry data. Table 10 presents the percent difference between the simulated data with respect to the measured one and data from CT Dosimetry. As mentioned before, the uncertainty for the experimental measurement was $8.1 \%$. Therefore, the compatibility between the simulated and measured data for both $C T D I_{100, c} / C T D I_{100, p}$ and $C T D I_{w} / C T D I_{100, a i r}$ ratios is achieved for body and head (with $10 \mathrm{~mm}$ of beam width) and body (with $40 \mathrm{~mm}$ of beam width) with a confidence interval of 6 times the standard deviation $( \pm 3 \sigma)$ to cover the $99.7 \%$ of the probabilities. In contrast, for the case of head CTDI phantom for $20 \mathrm{~mm}$ beam width, the discrepancy is exceeding the confidence interval for 6 times the standard deviation $( \pm 3 \sigma)$. The ratio $C T D I_{100, c} / C T D I_{100, p}$ evaluates the dose distribution inside the CTDI phantom and the ratio $C T D I_{w} / C T D I_{100, \text { air }}$ evaluates a CTDI dose descriptor with respect to the CT radiation output.

Table 8. Ratios of $C T D I_{100, c} / C T D I_{100, p}$ and $C T D I_{w} / C T D I_{100, a i r}$ for head and body CTDI phantoms and for tube potentials of $80,100,120$ and $140 \mathrm{kV}$. For all cases, the beam width was set to $n T=10 \mathrm{~mm}$. CTDosimetry (www.impactscan.org). $11.5 \%$ of uncertainty.

\begin{tabular}{|c|c|c|c|c|c|c|c|}
\hline & \multirow[b]{2}{*}{$\begin{array}{c}\text { Voltage } \\
(\mathbf{k V})\end{array}$} & \multicolumn{2}{|c|}{ Simulated } & \multicolumn{2}{|c|}{ Measured } & \multicolumn{2}{|c|}{ CTDosimetry } \\
\hline & & $\begin{array}{l}C T D I_{100, c} \\
C T D I_{100, p}\end{array}$ & $\begin{array}{c}C T D I_{w} / \\
C T D I_{100, a i r}\end{array}$ & $\begin{array}{l}C T D I_{100, c} \\
C T D I_{100, p}\end{array}$ & $\begin{array}{c}C T D I_{w} / \\
C T D I_{100, a i r}\end{array}$ & $\begin{array}{l}C T D I_{100, c} \\
C T D I_{100, p}\end{array}$ & $\begin{array}{c}C T D I_{w} / \\
C T D I_{100, a i r}\end{array}$ \\
\hline \multirow[t]{4}{*}{ Head } & 80 & 1.15 & 0.47 & 0.93 & 0.54 & 0.94 & 0.57 \\
\hline & 100 & 1.15 & 0.51 & 0.97 & 0.60 & 0.99 & 0.61 \\
\hline & 120 & 1.14 & 0.54 & 0.99 & 0.63 & 1.00 & 0.64 \\
\hline & 140 & 1.13 & 0.55 & 1.01 & 0.65 & 1.02 & 0.66 \\
\hline \multirow[t]{4}{*}{ Body } & 80 & 0.38 & 0.30 & 0.36 & 0.34 & 0.40 & 0.34 \\
\hline & 100 & 0.42 & 0.34 & 0.43 & 0.38 & 0.46 & 0.37 \\
\hline & 120 & 0.44 & 0.36 & 0.47 & 0.40 & 0.51 & 0.40 \\
\hline & 140 & 0.45 & 0.37 & 0.49 & 0.41 & 0.52 & 0.39 \\
\hline
\end{tabular}

Table 9. Ratios of $C T D I_{100, c} / C T D I_{100, p}$ and $C T D I_{w} / C T D I_{100, a i r}$ for head and body CTDI phantoms and for tube potentials of $80,100,120$ and $140 \mathrm{kV}$. The beam width was fixed to $n T=40 \mathrm{~mm}$ and $n T=20 \mathrm{~mm}$ for body and head CTDI phantom respectively. CTDosimetry (www.impactscan.org). 11.5\% of uncertainty.

\begin{tabular}{|c|c|c|c|c|c|c|c|}
\hline & \multirow[b]{2}{*}{$\begin{array}{c}\text { Voltage } \\
(\mathbf{k V})\end{array}$} & \multicolumn{2}{|c|}{ Simulated } & \multicolumn{2}{|c|}{ Measured } & \multicolumn{2}{|c|}{ CTDosimetry } \\
\hline & & $\begin{array}{l}C T D I_{100, c} / \\
C T D I_{100, p}\end{array}$ & $\begin{array}{c}C T D I_{w} / \\
C T D I_{100, a i r}\end{array}$ & $\begin{array}{l}C T D I_{100, c} / \\
C T D I_{100, p}\end{array}$ & $\begin{array}{c}C T D I_{w} / \\
C T D I_{100, a i r}\end{array}$ & $\begin{array}{l}C T D I_{100, c} / \\
C T D I_{100, p}\end{array}$ & $\begin{array}{c}C T D I_{w} / \\
C T D I_{100, a i r}\end{array}$ \\
\hline \multirow[t]{4}{*}{ Head } & 80 & 1.14 & 0.48 & 0.75 & 0.61 & 0.94 & 0.56 \\
\hline & 100 & 1.14 & 0.53 & 0.80 & 0.65 & 0.99 & 0.61 \\
\hline & 120 & 1.14 & 0.55 & 0.84 & 0.68 & 1.00 & 0.64 \\
\hline & 140 & 1.13 & 0.57 & 0.85 & 0.70 & 1.02 & 0.66 \\
\hline \multirow[t]{4}{*}{ Body } & 80 & 0.38 & 0.31 & 0.37 & 0.35 & 0.40 & -- \\
\hline & 100 & 0.41 & 0.34 & 0.41 & 0.38 & 0.46 & --- \\
\hline & 120 & 0.43 & 0.36 & 0.39 & 0.38 & 0.51 & --- \\
\hline & 140 & 0.45 & 0.38 & 0.49 & 0.40 & 0.52 & --- \\
\hline
\end{tabular}


Table 10. Percentage difference of $C T D I_{100, c} / C T D I_{100, p}$ and $C T D I_{w} / C T D I_{100, \text { air }}$ ratios between simulated, measured and values from CTDosimetry datasheet for tube potentials of 80, 100, 120 and $140 \mathrm{kV}$.

\begin{tabular}{|c|c|c|c|c|c|c|}
\hline \multirow[b]{3}{*}{ CTDI phantom } & \multirow[b]{3}{*}{$\begin{array}{c}n T \\
(\mathbf{m m})\end{array}$} & \multirow[b]{3}{*}{$\begin{array}{c}\text { Voltage } \\
(\mathbf{k V})\end{array}$} & \multicolumn{4}{|c|}{$\Delta \%$} \\
\hline & & & \multicolumn{2}{|c|}{ Simulated/Measured } & \multicolumn{2}{|c|}{ Simulated/CTDosimetry } \\
\hline & & & $\begin{array}{l}C T D I_{100, c} \\
C T D I_{100, p}\end{array}$ & $\begin{array}{c}C T D I_{w} / \\
C T D I_{100, a i r}\end{array}$ & $\begin{array}{l}C T D I_{100, c} \\
C T D I_{100, p}\end{array}$ & $\begin{array}{c}C T D I_{w} / \\
C T D I_{100, a i r}\end{array}$ \\
\hline \multirow{8}{*}{ Head } & \multirow{4}{*}{10} & 80 & 23.4 & 14.0 & 21.9 & 17.8 \\
\hline & & 100 & 18.5 & 13.8 & 16.2 & 16.4 \\
\hline & & 120 & 15.1 & 14.1 & 14.1 & 16.1 \\
\hline & & 140 & 12.7 & 14.4 & 10.9 & 15.8 \\
\hline & \multirow{4}{*}{20} & 80 & 52.9 & 21.4 & 21.8 & 14.5 \\
\hline & & 100 & 41.6 & 19.4 & 15.6 & 13.6 \\
\hline & & 120 & 35.4 & 18.1 & 13.6 & 13.4 \\
\hline & & 140 & 32.0 & 18.0 & 10.5 & 13.2 \\
\hline \multirow{8}{*}{ Body } & \multirow{4}{*}{10} & 80 & 6.1 & 11.7 & 5.0 & 10.9 \\
\hline & & 100 & 3.9 & 10.3 & 9.0 & 8.2 \\
\hline & & 120 & 6.8 & 9.8 & 13.6 & 10.1 \\
\hline & & 140 & 8.9 & 8.8 & 13.8 & 5.6 \\
\hline & \multirow{4}{*}{40} & 80 & 2.2 & 11.5 & 5.8 & --- \\
\hline & & 100 & 1.0 & 10.9 & 10.0 & --- \\
\hline & & 120 & 11.6 & 4.0 & 14.6 & --- \\
\hline & & 140 & 8.0 & 5.5 & 14.5 & --- \\
\hline
\end{tabular}

\subsubsection{Validation based on dose profiles}

Theoretical dose profiles derived by the analytical formulation described in Section 2.3, and measured dose profiles obtained with the experimental procedure described in Section 3.3.2 are used to validate the dose profiles computed with MC simulation.

First, a brief description of the analytical profiles with comments about their characteristics is presented in Section 4.1.2.1. Second, measured primary and total dose profiles for head and body CTDI phantoms are shown in Section 4.1.2.2. The totally of the experimental dose profiles are presented in Appendix B. Finally, in Section 4.1.2.3 the corresponding evaluation of the simulated dose profiles is described.

\subsubsection{Analytical Dose Profiles}

The analytical equations corresponding to the primary, scatter and total dose profiles were implemented in MatLab R2012a environment (The MathWorks1 Inc., Natick, MA, 2012). Figure 46 shows the user interface developed to compute automatically the dose profiles. As mentioned, a dose profile could be understood as the contribution of two components named primary profile and scatter profile. Figure 43.a shows primary profiles computed for values of 40, 30, 20 and $10 \mathrm{~mm}$ of the beam width, $a$, onto the AOR, and for a focal spot angle of $\varphi=7^{\circ}$. Figure 43.b shows primary profiles plotted for six values of the focal spot angle, $\varphi$, and $a=20 \mathrm{~mm}$. All profiles shown in Figure 43 were computed using 
Equation (32). The primary profile allows to characterize some elements of the X-ray tube system and geometric characteristics of the CT system, since that the primary profile shape is influenced by the heel effect, the angle of the anode surface, the size of the focal spot, and the $\mathrm{X}$-ray spectrum as well as by the CT magnification factor defined as, $M=F / F_{c}$. In Figure 43.a, it is presented the effects of the focal spot size in the primary profile, since that the focal spot is not a point source, the actual X-ray beam profile along the z-axis has at its both sides a penumbra which impact the shape of the primary profile (see, Figure 15.b and Figure 15.c for better understanding). Penumbra is a beam's region with low intensity with respect to the central region of the beam, as a consequence, the central region of the primary profile is illuminated by a major photon intensity than its initial and final part, also the penumbra presented at each side of the beam are not symmetric due to the tilted focal spot (see, Figure 24 for better understanding). Another important characteristics of the primary profile is its tilted top part, this issue is mainly related to the heel effect (see, subsection 2.1.2 for details) and also has influence of the X-ray spectrum and the anode surface angle. It is interesting to note that it is possible to identify indirectly from the shape of the primary profile, from what direction the electron beam strike the anode surface. As the low intense part of the primary profile correspond to the target side, therefore, the electron beam comes to strike the target surface from the left to right on the z-axis in the figures. In Figure 43.b, it can be identified the influence of the angle, $\varphi$, (see, Figure 13 for definition of, $\varphi$ ) on the primary profile shape. The impact of the heel effect on primary profile depend on, $\varphi$, for lower angles, the upper part of the primary profile become more tilted, in contrast, for higher angles the upper part become flatter. It is interesting to note that the analytical equation for the primary profile models with consistent the non-equal influence of the heel effect at lower angles on the two half parts of the primary profile in Figure 43.b, for instance, at $\varphi=2^{o}$, the half upper part at right is more affected by the heel effect then the other one at left. The angle, $\varphi$, also affects the penumbra, for higher angles the penumbras become more symmetric rather than for lower angles in which the penumbra at the left side is more pronounce than the one at the right side. The primary dose profile presented in Figure 43.a and Figure 43.b were computed on the central axis of a CTDI phantom and normalized to the unit at $z=0$ to become more practical the comparison, they correspond to a $120 \mathrm{kV}$ X-ray spectrum. The primary profile shape is unchanged if the head or body CTDI phantom were used in the profile plotting, actually, it is also the same if is measured free-in-air as stated by Dixon et al. "therefore, the primary beam 
profile on the phantom axis of rotation will have the same relative shape as that in air with no phantom present"(Dixon et al., 2005).
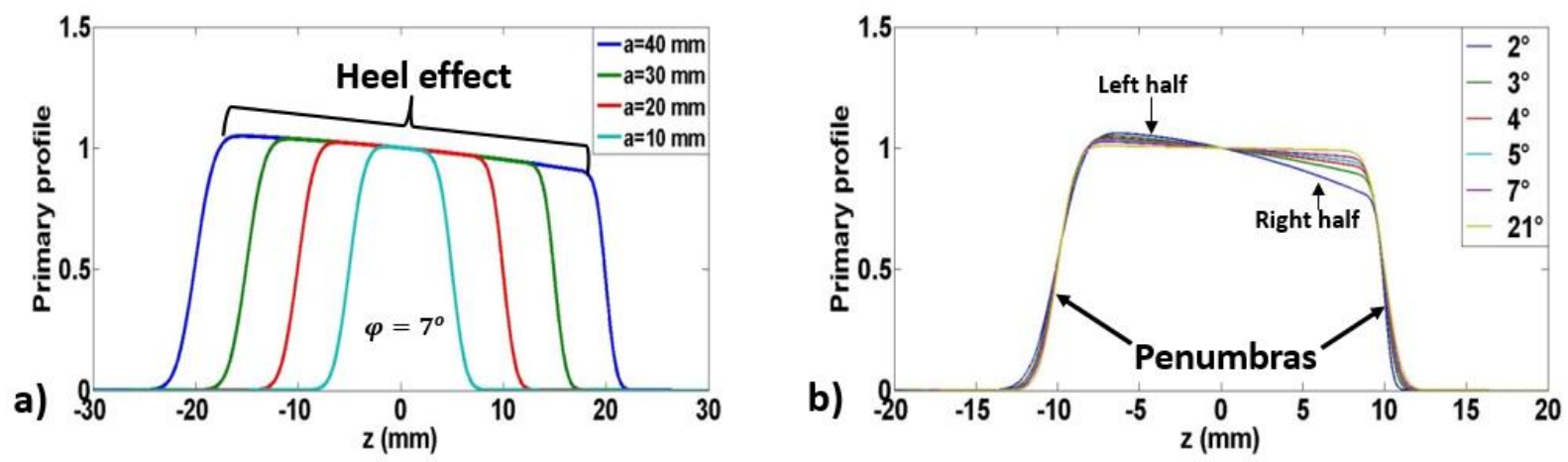

Figure 43. a) Primary profiles for four values, $40,30,20$ and $10 \mathrm{~mm}$, of the beam width onto the AOR, $a$, at $\varphi=7^{\circ}$, the impact of the heel effect on the profile is indicated. b) Primary profiles computed for six values of the tilting angle of the focal spot, at $a=20 \mathrm{~mm}$. The penumbra at both sides are indicated, also the influence of the heel effect when $\varphi=7^{\circ}$ at the left and right halves of the profile's upper part is indicated as well. All primary profiles were normalized to the unit at $\mathrm{z}=\mathbf{0}$, for better comparison, they were computed along the central axis of a CTDI phantom.

Figure 44 shows analytical scatter profiles computed for values of 138, 30, 20 and $10 \mathrm{~mm}$ of the beam width, $a$, onto the AOR for focal spot angle of $\varphi=7^{\circ}$. The analytical model divides into two parts the scatter profile, the one who is inside the beam shadow and the other one who is outside the beam shadow. In Figure 44, the color central region in each profile is inside the beam shadow and the two black regions are both out the beam shadow. Equation (33) and Equation (34) correspond to regions inside and outside of the beam shadow, respectively. Scatter profiles in Figure 44 were computed on the central axis of a PMMA cylindrical phantom of $32 \mathrm{~cm}$ diameter and for $120 \mathrm{kV}$ X-ray spectrum. Differently that in the primary profile case, in which the relative shape is the same for both CTDI phantoms, in the case of scatter profiles their intensities and shapes are determined by the theoretical Compton-scattering equations, which depend of the phantom attenuation.

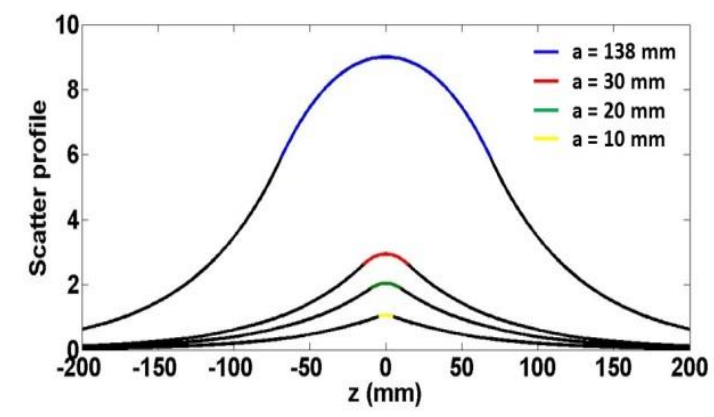

Figure 44. Scatter profiles for values of $138,30,20$, and $10 \mathrm{~mm}$ of the beam width, $a$, onto the AOR with a focal spot angle of $\varphi=7^{\circ}$. The central color region in each profile is inside the beam shadow and the black regions at both sides are out the beam shadow. 
The sum of the primary and scatter components produces the complete dose profile as presented in Figure 45. They were computed for values of 100, 80, 50 and $20 \mathrm{~mm}$ of the beam width, $a$, onto the AOR and for a focal spot angle of $\varphi=7^{\circ}$. These dose profiles model the dose distribution on the central axis of a body CTDI phantom. They were normalized to the unit at $\mathrm{z}=0$ and correspond to a $120 \mathrm{kV} \mathrm{X}$-ray spectrum.

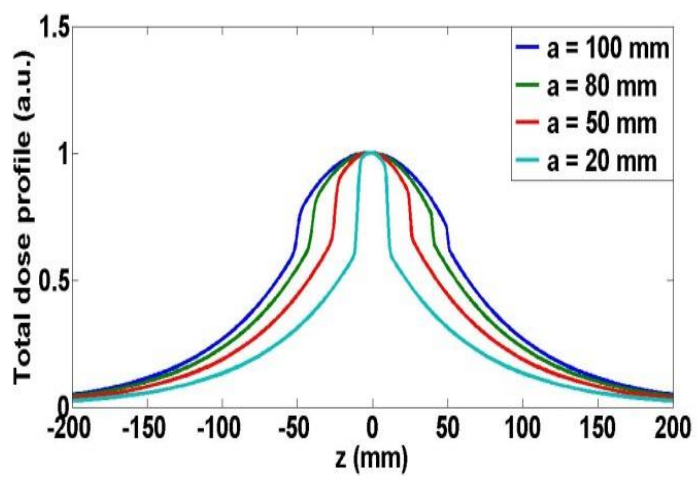

Figure 45. Total dose profile for values of $100,80,50$, and $20 \mathrm{~mm}$ of the beam width, $a$, onto the AOR with a focal spot angle of $\varphi=7^{\circ}$. They are normalized to the unit at $\mathrm{z}=\mathbf{0}$.

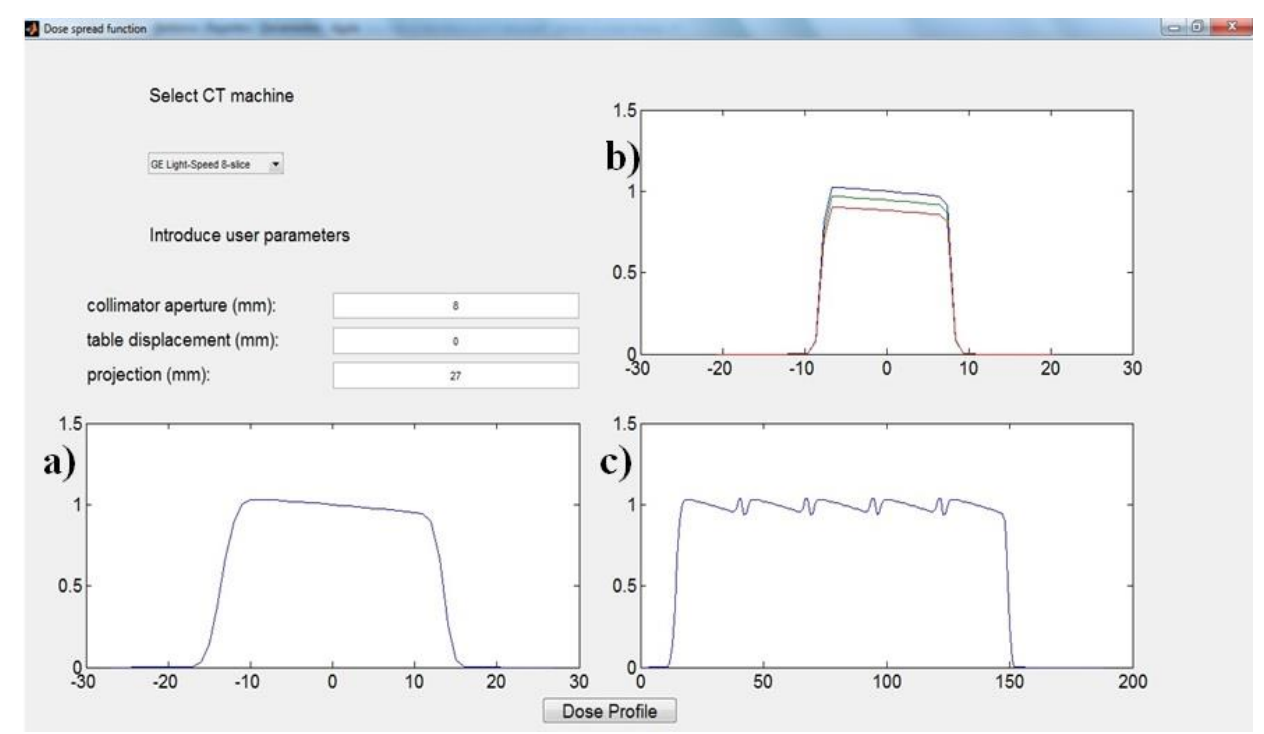

Figure 46. User interface program implemented in MatLab R2012a environment (The MathWorks1 Inc., Natick, MA, 2012) showing the primary dose profile for a) central, b) peripheral phantom axis and c) the cumulative primary dose profile for central axis.

\subsubsection{Experimental Dose Profiles}

Section 3.3.2 described the experimental methodology employed to measure the primary and total profiles using OSL ribbons in the GE Discovery CT750 HD scanner (General Electric Company, Boston, USA). Figure 47 presents primary profiles from 
experimental measurements on the CT AOR for X-ray tube voltages of 80, 100, 120 and $140 \mathrm{kV}$ with the head bowtie filter setting. In Figure 47.a and Figure 47.b the nominal beam width, $n T$, was set to $20 \mathrm{~mm}$ and $40 \mathrm{~mm}$, respectively. Note that, $n T$, is an image parameter and it is not equal to the beam width, $a$, on the AOR.
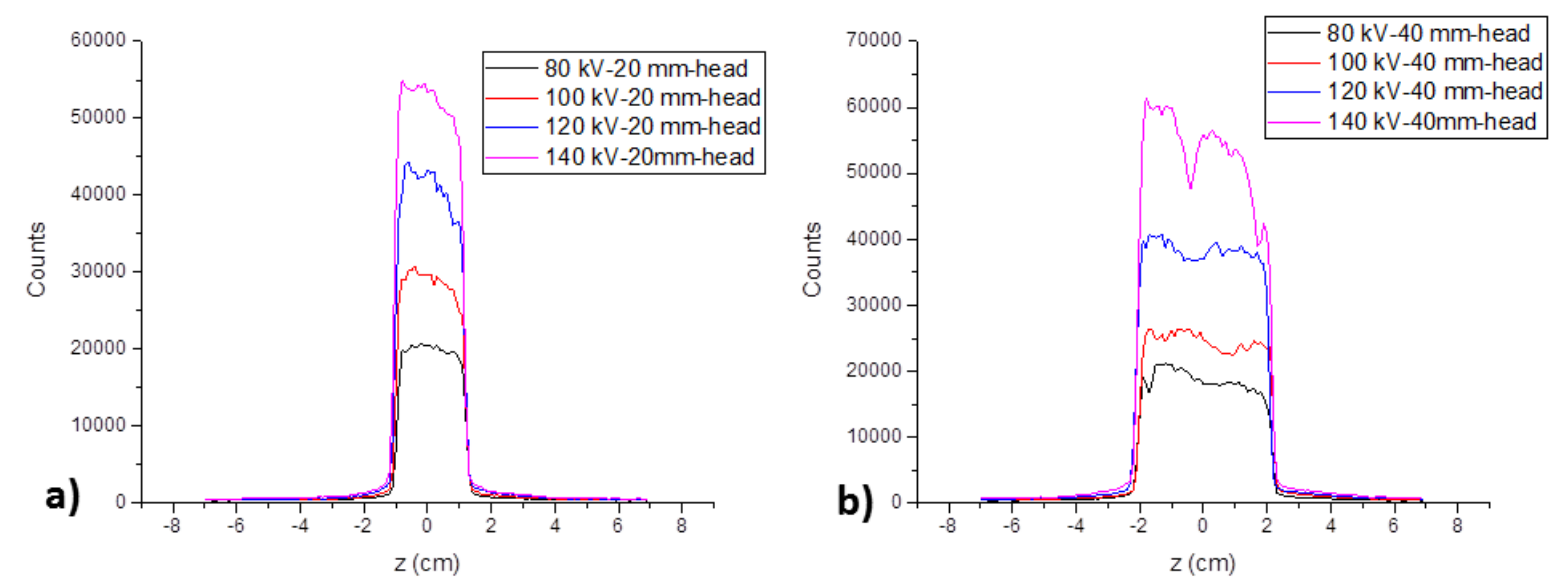

Figure 47. Primary profiles for head bowtie filter at nominal beam width of a) $n T=20 \mathrm{~mm}$ and b) $n T=40 \mathrm{~mm}$. Measured using OSL ribbons for 80, 100, 120, and $140 \mathrm{kV}$ for both cases.

Figure 48 presents primary profiles from experimental measurements on the CT AOR for X-ray tube voltages of $80,100,120$ and $140 \mathrm{kV}$ with the body bowtie filter setting. In Figure 48.a and Figure 48.b the nominal beam width, $n T$, was set to $40 \mathrm{~mm}$, but, in the second case the width of the profiles are clearly broader than those in Figure 48.a. The acquisitions in the second case were performed in the CT service mode which possibly affected the collimator aperture. Note that, in the same way that in Figure $47, n T$, is an image parameter and it is not equal to the beam width, $a$, on the AOR.
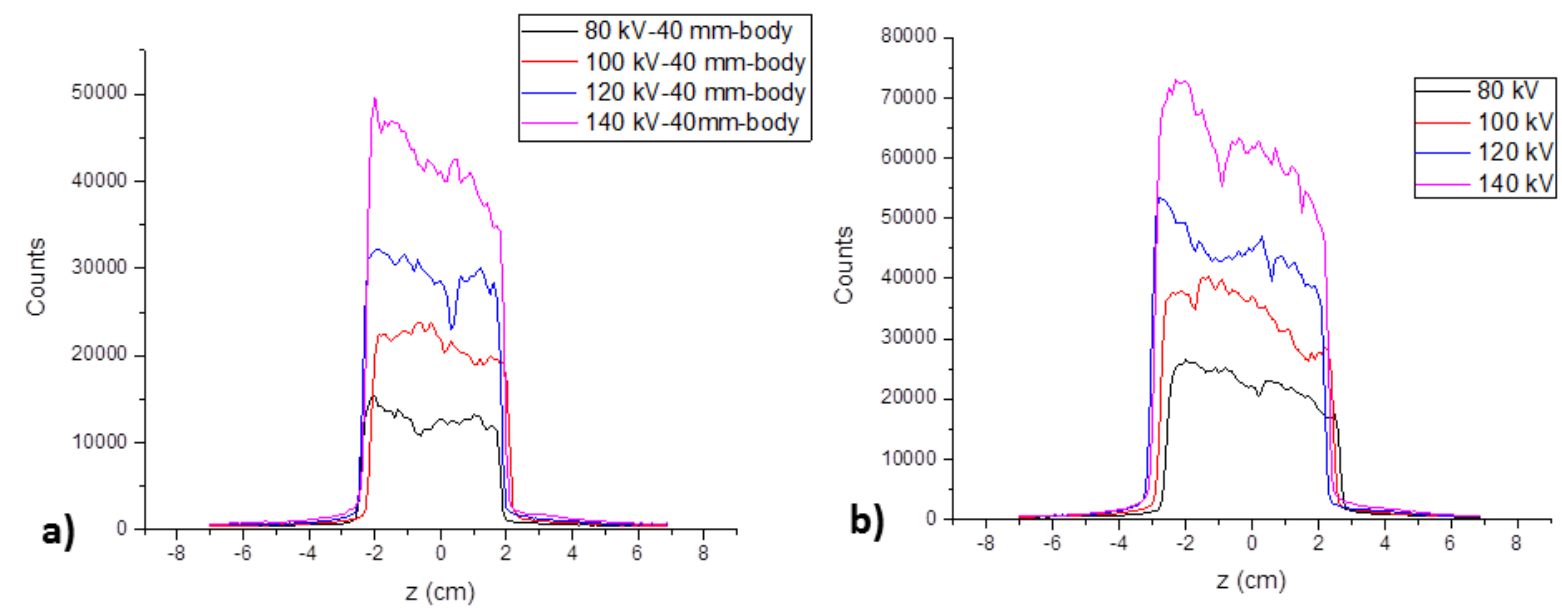

Figure 48. Primary profiles for body protocol (body bowtie filter) at nominal beam width of a) $n T=40 \mathrm{~mm}$ and b) $n T=40 \mathrm{~mm}$. Measured using OSL ribbons. 
As the analytical primary profiles in Figure 43, the shape of the primary profiles in Figure 47 and Figure 48 reflect the influence of the heel effect, focal spot size, and the tilted target surface in the GE Discovery CT750 HD scanner (see, Table 2 for scanner details). Since the X-ray tube workload was $2000 \mathrm{mAs}$ for all cases, the intensity in each set of primary profiles are related to the tube voltage, at higher tube voltage the primary profile is more intense, in contrast, at lower tube voltage the primary profile is less intense. Approximately, the X-ray fluence is proportional to the square of the tube voltage. Figure 49 shows a comparison of primary dose profiles from measurements using OSL ribbons, computed from analytical equations, and computed from MC simulation. Table 11 presents the over-beaming factor $a / n T$, where $a$ is the beam width onto the AOR, determined from primary dose profiles measured in the GE Discovery CT750 HD, and $n T$ is the nominal beam width and compared with the over-beaming factor for the GE VCT scanner (Dixon and Ballard, 2007). From Table 11, 5\% and 10\% of differences between $a$ and $n T$ were identified for $n T=40 \mathrm{~mm}$ and $n T=20 \mathrm{~mm}$, respectively. These values are close to those measured by Dixon et al. in the GE VCT scanner that found $5.3 \%$ and $12 \%$ of differences between $a$ and $n T$ for $n T=40 \mathrm{~mm}$ and $n T=20 \mathrm{~mm}$, respectively. From Table 11 , it can be deduced that the Xray beam illuminates region beyond the limits of the nominal detector length. In the $\mathrm{MC}$ model of the helical scanning, the over-beaming was not introduced. Therefore, in the MC simulation of the dose profiles, the nominal beam width $n T$ and the beam width $a$ have the same values. The no introduction of the over-beaming in the MC modelling of the helical scan mode may impact the shape of the cumulated dose profile and it could appear slightly different with respect to the measured one. Nevertheless, since the cumulative dose profiles were calibrated considering the experimental values of the $C T D I_{100}$ that actually are affected by the over-beaming effect, CT dose metrics presented in this work derived from the calibrated cumulative profiles should not be hard affected by the no-introduced over-beaming effect. 

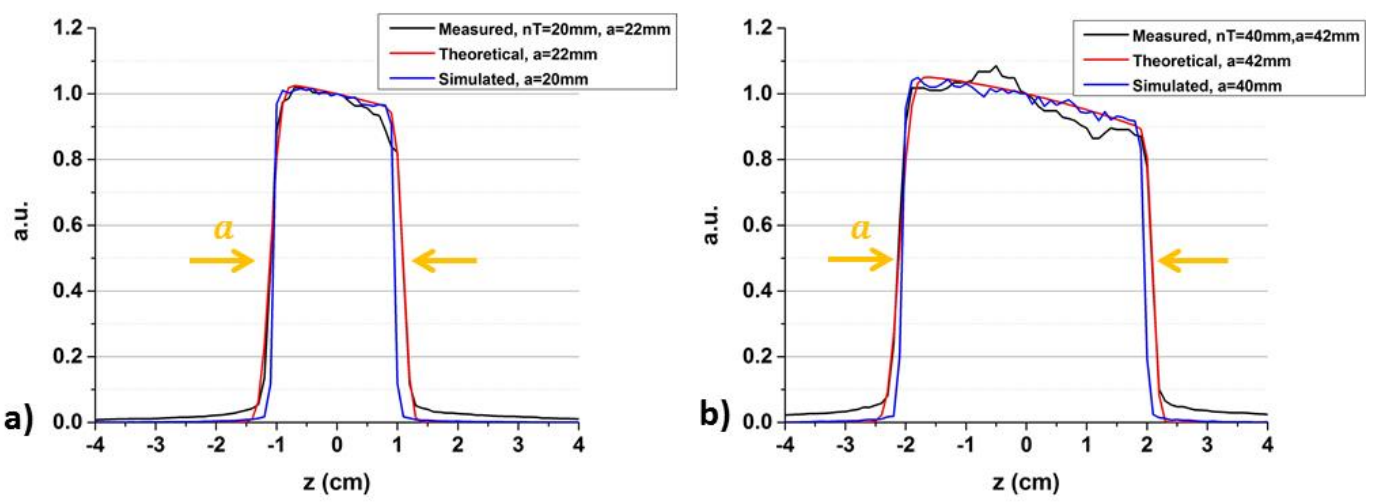

Figure 49. Measured, theoretical, and simulated primary dose profiles a) for head bowtie filter at nominal beam width $n T=20 \mathrm{~mm}$ and b) body bowtie filter at nominal beam width $n T=40 \mathrm{~mm}$. Experimental dose profiles were measured using OSL ribbons.

Table 11. $a$ collimator aperture projected onto the AOR, $a / n T$ is the over-beaming factor. Measured for this work on GE Discovery CT750 HD scanner, (Dixon and Ballard, 2007) on GE VCT scanner.

\begin{tabular}{lccccc} 
& & \multicolumn{3}{c}{ Large focal spot size } \\
\cline { 3 - 6 } & & \multicolumn{2}{c}{ This work } & \multicolumn{2}{c}{$\begin{array}{c}\text { Dixon and } \\
\text { Ballard, 2007) }\end{array}$} \\
\hline $\begin{array}{l}\text { Acquisition } \\
\text { Collimator } \\
\boldsymbol{n} \boldsymbol{x} \boldsymbol{T}\end{array}$ & $\begin{array}{c}\boldsymbol{n T} \\
(\mathrm{mm})\end{array}$ & $\begin{array}{c}\boldsymbol{a} \\
(\mathrm{mm})\end{array}$ & $\boldsymbol{a} / \boldsymbol{n T}$ & $\begin{array}{c}\boldsymbol{a} \\
(\mathrm{mm})\end{array}$ & $\boldsymbol{a} / \boldsymbol{n T}$ \\
\hline $\mathbf{6 4 x 0 . 6 2 5}$ & 40 & $42.0(5)$ & 1.05 & 42.13 & 1.053 \\
$\mathbf{3 2 \times 0 . 6 2 5}$ & 20 & $22.0(5)$ & 1.10 & 22.39 & 1.12 \\
$\mathbf{6 4 x 0 . 6 2 5}$ & 40 & $52.0(5)$ & 1.30 & --- & -- \\
\hline
\end{tabular}

In addition to the primary profiles, measurements of the dose profiles in head and body CTDI phantoms were carried out in order to be used to validate the simulated profiles. Dose profiles for tubes voltages of 80,100, 120 and $140 \mathrm{kV}$ were measured for head and body CTDI phantoms. The choice of the nominal beam width, $n T$, followed the common CT protocols in the clinic listed in Table 6. A CTDI phantom has 5 cavities where the pencil ion chamber is introduced to measure the $C T D I_{100}$. The holes are one along the central axis and four along the peripheral axes (at $1 \mathrm{~cm}$ from the phantom surface) of the CTDI phantom, these holes are used to introduce the OSL ribbons to measure the dose profiles as described in Section 3.3.2. Figure 50 shows dose profiles measured in the head and body CTDI phantoms, which are calibrated in term of air kerma and they are presented units of (mGy/100mAs). The dose profile calibration is performed using its corresponding $C T D I_{100}$ value, see Figure 42 for details. Figure 50.a presents five dose profiles measured in the head CTDI phantom at $n T=20 \mathrm{~mm}$, and Figure 50.b, presents four dose profiles measured in the body CTDI phantom 
for $n T=40 \mathrm{~mm}$. The intensity of each dose profile in Figure 50 reflects its corresponding $C T D I_{100}$ value. Despite that in Figure 50.a the central dose profile appear to be less intense than the others, their total integration may have roughly the same values ${ }^{t}$. In the case of Figure $50 . \mathrm{b}$, the central dose profile is in fact less intense than the ones at the periphery ${ }^{\mathrm{u}}$ mainly due to the phantom material attenuation. The alignments of the CTDI phantoms were performed on the CT table (see, Section 3.3.1 for explanation). In fact, the attenuation of the patient table has influence on the measurement of the $C T D I_{100}$, especially in the position three, which is the one that is closest to the CT table and, therefore, is more affected by it. This situation can be visualized in Figure 50, in both cases head and body phantom, nevertheless, the effects on the 3-position profiles in the body phantom is more evidence.
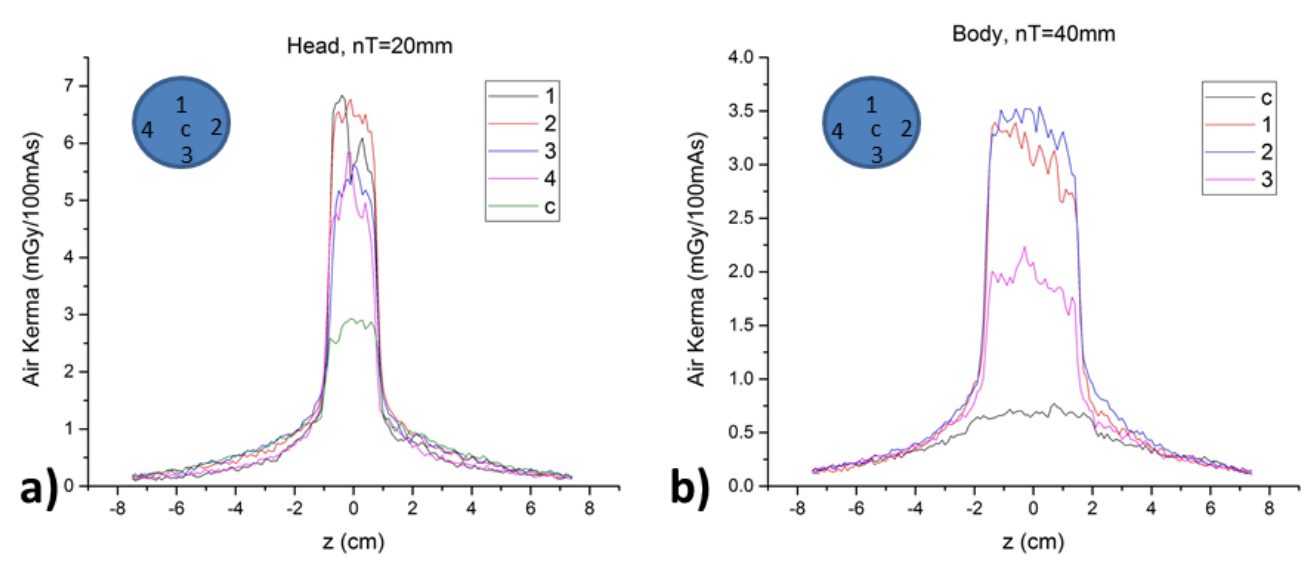

Figure 50. Dose profiles for a) head protocol at $n T=20 \mathrm{~mm}$ and a) body protocol at $\boldsymbol{n T}=\mathbf{4 0} \mathbf{m m}$. Measured using OSL ribbons at $120 \mathrm{kV}$.

Before the calibration of the measured dose profiles into dosimetric units, a subtraction of the radiation background (BG) signal were performed. Figure 51 shows four cases of radiation background distribution which come from the reading of the OSL ribbons without irradiation. Only, one of them produced a major BG level, which in mean delivered 255 counts, the others three produced approximately 129 counts. Depending the behavior of the dose profiles reading, it was chosen one of those values to be subtracted from each dose profile bin.

\footnotetext{
${ }^{\mathrm{t}}$ The $C T D I_{100}$ values for the head phantom should be approximately the same at central and peripheral positions.

${ }^{u}$ The $C T D I_{100, c}$ value for the body phantom should be approximately a half of the $C T D I_{100, p}$ value.
} 


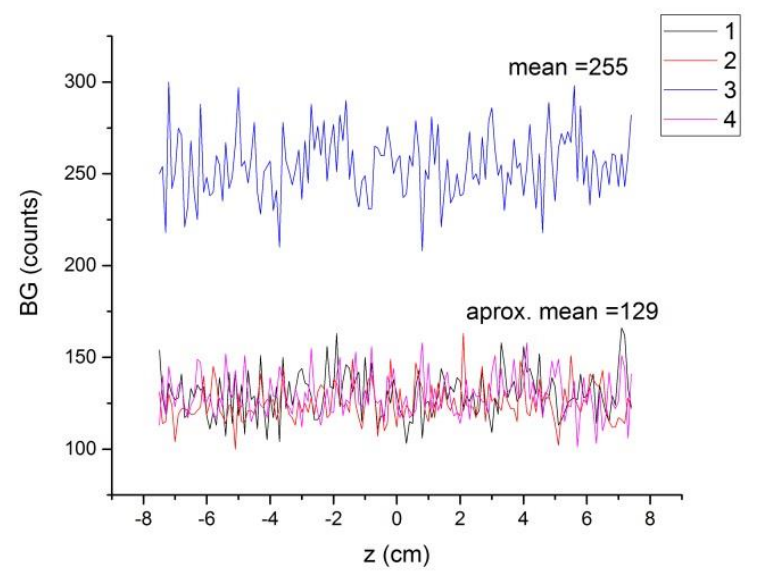

Figure 51. Radiation background (BG) of dose profiles measurements.

\subsubsection{Validation of the simulated dose profiles}

Primary and total dose profiles along the central and peripheral axes of a CTDI phantom were computed using the X-ray source model. The source model has been implemented to include the focal spot angle, the target self-attenuation, X-ray spectrum and the bowtie filter. Special attention was given in this work to the primary component of the dose profile, since it allows the evaluation of the CT device geometry, X-ray spectrum and target self-attenuation. Figure 52 shows the comparison between analytical and simulated primary profiles for produced by beam widths, $a$, of 10, 20, and $27 \mathrm{~mm}$ onto the AOR. The collimator apertures $w$ could be computed from $a=M . w$, where, $M$ is the magnification factor defined by $M=F / F_{c}, F$ is the source to isocenter distance and $F_{c}$ is the source to collimator distance. For instance, in the GE Discovery CT750 HD, F $=541 \mathrm{~mm}$ and $F_{c}=162 \mathrm{~mm}$, as showed in Table 2 . The primary dose profile with $27 \mathrm{~mm}$ width has a collimator aperture of $w=8.08 \mathrm{~mm}$ and magnification of $M=3.34$. The primary profiles, $f_{p}(z)$, are presented as a function of the z-coordinate and they were normalized by $f_{p}(0)$, dose profile at $z=0$. The simulation setup used to compute the primary dose profile is the same that is shown Figure 29, but, the CTDI phantom is withdrawal to just put a cylindrical body with $1 \mathrm{~cm}$ diameter as photon absorbed, as is also shown in Figure 30. The photon cutoff energy was set at $150 \mathrm{keV}$ in order to absorb all photons delivered to the $1 \mathrm{~cm}$ diameter body to avoid secondary particles and improve the photon statistics. 


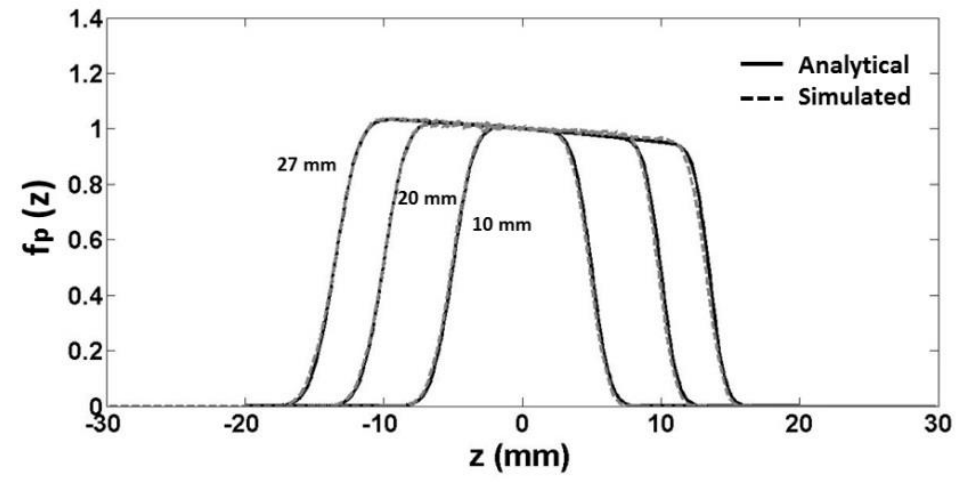

Figure 52. Analytical (continuous) and simulated (dashed) primary profiles beam width, $a$, of 10,20 and $27 \mathrm{~mm}$ onto the AOR.

Simulated primary profile presents asymmetric penumbra due to the focal spot angle and asymmetric energy deposition at the upper part of the profile due to the target selfattenuation (heel effect). The collimator aperture, $w$, from the simulation reproduce adequately the beam width of 10,20 , and $27 \mathrm{~mm}$, the penumbra area (both sides) and the upper part of the profile are in accordance.

As mentioned in Section 2.3, the dose profile is the sum of two components named as primary and scatter components. The primary component is generated by the primary photons transmitted through the phantom material and impinging on the radiation detector inside the phantom (ion chamber or OSL strip). The scatter component appears from the deposited dose due to the detection of photons from the primary beam scattered by the phantom material and impinging on the radiation detector. Figure 53.a compares analytical and simulated dose profiles which were produced from beam widths of 10, 20 and $27 \mathrm{~mm}$ of thickness on the central axis of a cylindrical phantom. The analytical equations are described in detail in Section 2.3, specifically, Equation (32) computed the primary profile, Equations (33) and Equation (34) computed the scatter profile and Equation (17) compute the dose profile. Simulated dose profiles shown in Figure 53 were computed along the central axis of a simulated large body CTDI phantom from -200 to $200 \mathrm{~mm}$ onto the z-axis. Figure 53.b illustrates the contribution of the primary and scatter components on the dose profile and comparison with simulated profile for $27 \mathrm{~mm}$ of beam width. The simulation was performed for X-ray spectrum of $120 \mathrm{kV}$. 

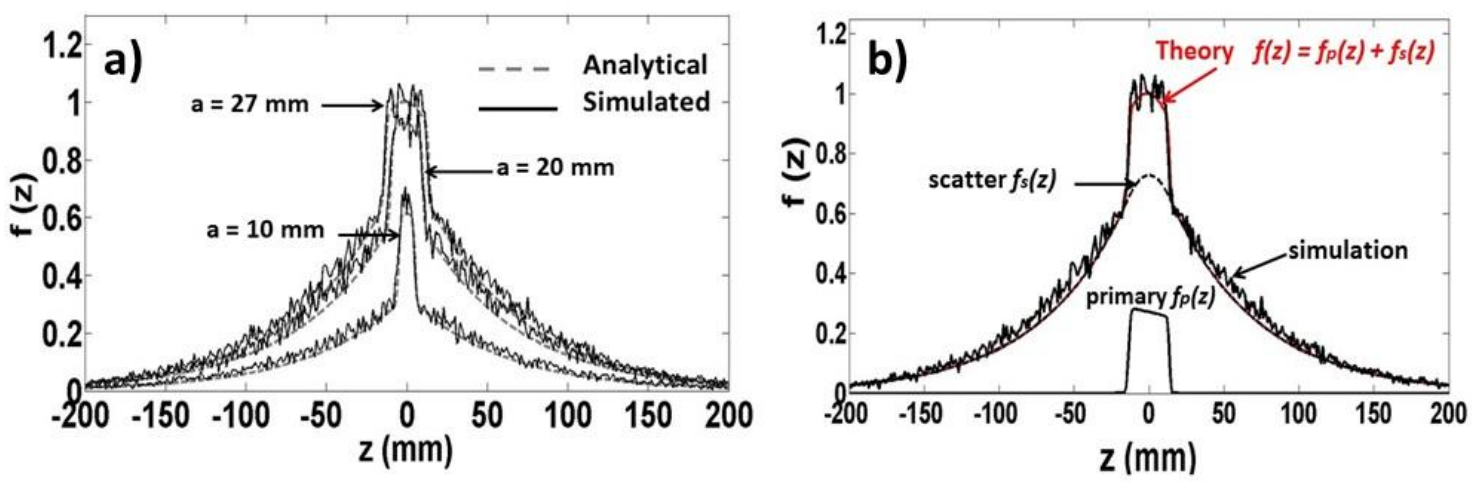

Figure 53. (a) Comparison of analytical and simulated dose profile for 10,20 and $27 \mathrm{~mm}$ of collimation projected onto the AOR. (b) Contribution of primary and scatter component on the dose profile for a cylindrical phantom with $32 \mathrm{~cm}$ diameter at $120 \mathrm{kV}$.

The validation of the simulated dose profiles is also performed using the measured dose profiles for a single axial irradiation. The employed method used to calibrate the both experimental and simulated dose profile is described in Figure 42, in which the experimental $C T D I_{100}$ at central and peripheral (1-position) axes were taking into account to convert the units (eV/g.hist) into (mGy/100mAs) for simulated profiles and to convert the units (counts) into $(\mathrm{mGy} / 100 \mathrm{mAs})$ for experimental profiles. Since the length of the pencil ion chamber used to measure the $C T D I_{100}$ is $10 \mathrm{~cm}$, the calibration of both simulated and measured profiles force that the integration in the interval $(-5 \mathrm{~cm}, 5 \mathrm{~cm})$ may result in the corresponding $C T D I_{100}$ value, for the central and peripheral positions. The conversion coefficient for profile calibrations for both head and body CTDI phantoms are shown in Table 12 and Table 13, respectively. There are not uncertainties for the conversion coefficients shown in Table 12 and Table 13, uncertainties for the simulated profiles comes from the MC computation.

Table 12. Conversion coefficients to convert the dose profiles units from deposited dose per simulated history to Air-Kerma (mGy/100 mAs). Simulation over the head CTDI phantom with nT=20 mm.

\begin{tabular}{ccccccc}
\hline $\begin{array}{c}\text { Voltage } \\
(\mathbf{k V})\end{array}$ & $\begin{array}{c}\text { Deposited Dose } \\
(\mathbf{e V} / \mathbf{g} \text { hist})\end{array}$ & \multicolumn{2}{c}{$\begin{array}{c}\text { Air-Kerma } \\
(\mathbf{m G y} / \mathbf{1 0 0} \mathbf{m A s})\end{array}$} & \multicolumn{2}{c}{$\begin{array}{c}\text { Conversion coefficients } \\
(\mathbf{m G y} \text { g hist/100 } \mathbf{~ m A s ~} \mathbf{~ e V})\end{array}$} \\
\cline { 2 - 7 } & Central & Peripheral & Central & Peripheral & Central & Peripheral \\
\hline 80 & 229.6 & 200.1 & 1.12 & 1.49 & 0.49 & 0.74 \\
100 & 255.9 & 224.8 & 2.11 & 2.62 & 0.82 & 1.17 \\
120 & 280.2 & 245.2 & 3.32 & 3.96 & 1.19 & 1.61 \\
140 & 301.2 & 266.2 & 4.72 & 5.52 & 1.57 & 2.07 \\
\hline
\end{tabular}


Table 13. Conversion coefficients to convert the dose profiles units from deposited dose per simulated history to Air-Kerma (mGy/100 mAs). Simulation over the body CTDI phantom with $\mathrm{nT}=40 \mathrm{~mm}$.

\begin{tabular}{ccccccc}
\hline $\begin{array}{c}\text { Voltage } \\
(\mathbf{k V})\end{array}$ & $\begin{array}{c}\text { Deposited Dose } \\
(\mathbf{e V} / \mathbf{g} \text { hist})\end{array}$ & \multicolumn{2}{c}{$\begin{array}{c}\text { Air-Kerma } \\
(\mathbf{m G y} / \mathbf{1 0 0} \mathbf{~ m A s})\end{array}$} & \multicolumn{2}{c}{$\begin{array}{c}\text { Conversion coefficients } \\
(\mathbf{m G y} \mathbf{g} \text { hist/100 } \mathbf{~ m A s} \mathbf{~ e V})\end{array}$} \\
\cline { 2 - 6 } & Central & Peripheral & Central & Peripheral & Central & Peripheral \\
\hline 80 & 32.7 & 86.7 & 0.5 & 1.34 & 1.53 & 1.55 \\
100 & 39.9 & 96.5 & 1.08 & 2.64 & 2.71 & 2.74 \\
120 & 46.0 & 105.3 & 1.57 & 4.04 & 3.41 & 3.84 \\
140 & 51.3 & 114.1 & 2.8 & 5.77 & 5.45 & 5.06 \\
\hline
\end{tabular}

Figure 54, 55, 56, and 57 show the one by one comparisons between measured and simulated profiles determined in a head CTDI phantom for the central and peripheral (1position) axes. In the same way, Figure 58, 59, 60, and 61 show the one by one comparisons between measured and simulated profiles determined in a body CTDI phantom for the central and peripheral (1-position) axes. The procedure comprises four X-ray tube voltages, 80, 100, 120 , and $140 \mathrm{kV}$, and the imaging parameter, $n T$, were set to $20 \mathrm{~mm}$ and $40 \mathrm{~mm}$ for head and body CTDI phantom, respectively, according to Table 6 . All dose profiles are in terms of air kerma with units of $(\mathrm{mGy} / 100 \mathrm{mAs})$. The conversion coefficients shown in Table 12 were used for the calibration. The uncertainty of the experimental $C T D I_{100}$ set in $8.1 \%$ was not considered for the profile calibrations. In contrast, Poisson uncertainty for the measured profile and the uncertainty from the MC simulation for the simulated profile were represented in the error bars in the figures. In Figure 54, 55, 56, and 57, the full width and half maximum, FWHM, for both central and peripheral profiles appear to be in accordance for the simulated and measure profiles. Same conclusion could be found for the Figure 58, 59, 60, and 61.
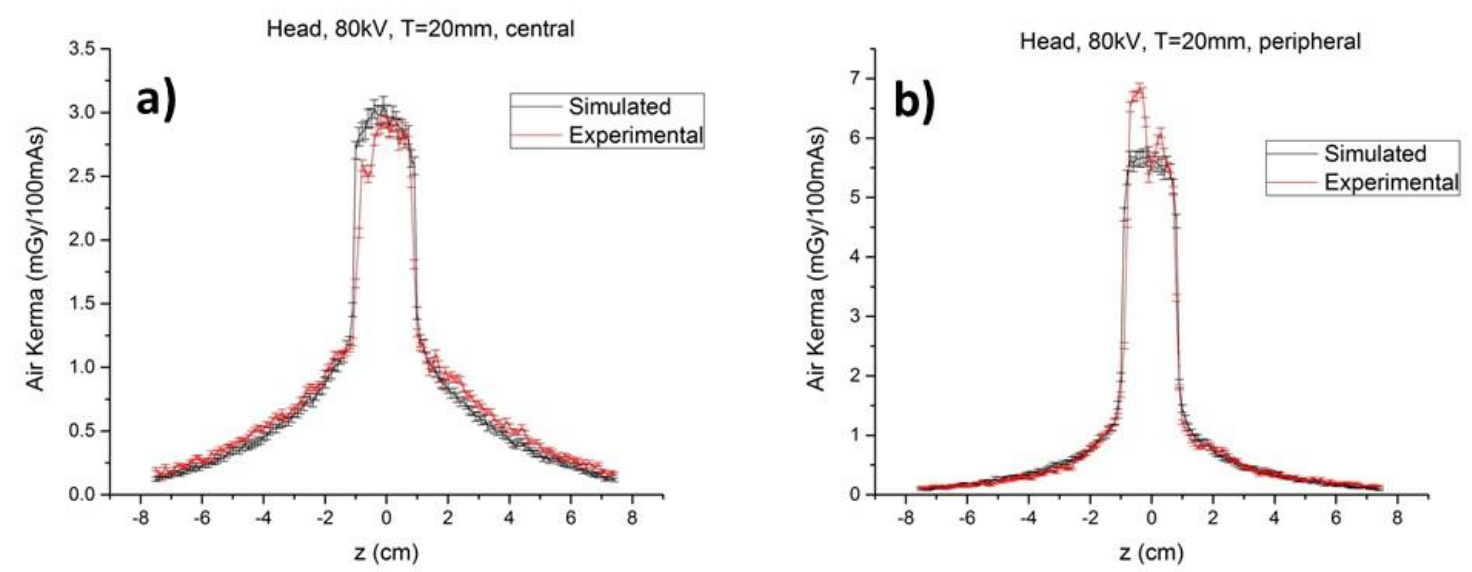
Figure 54. a) Central and b) Peripheral Dose Profile in Head CTDI phantom at 80kV and nT=20mm beam width. Comparison between simulated (black line) and experimental (red line) Dose Profiles.
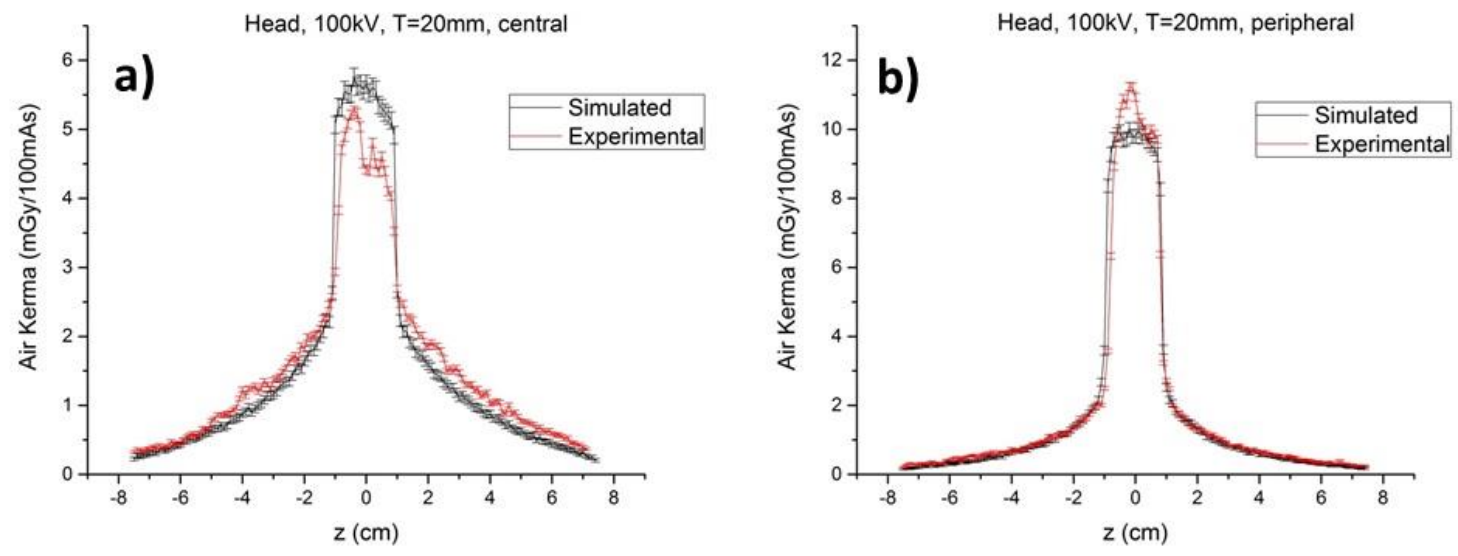

Figure 55. a) Central and b) Peripheral Dose Profile in Head CTDI phantom at $100 \mathrm{kV}$ and $\mathbf{n T}=\mathbf{2 0 m m}$ beam width. Comparison between simulated (black line) and experimental (red line) Dose Profiles.
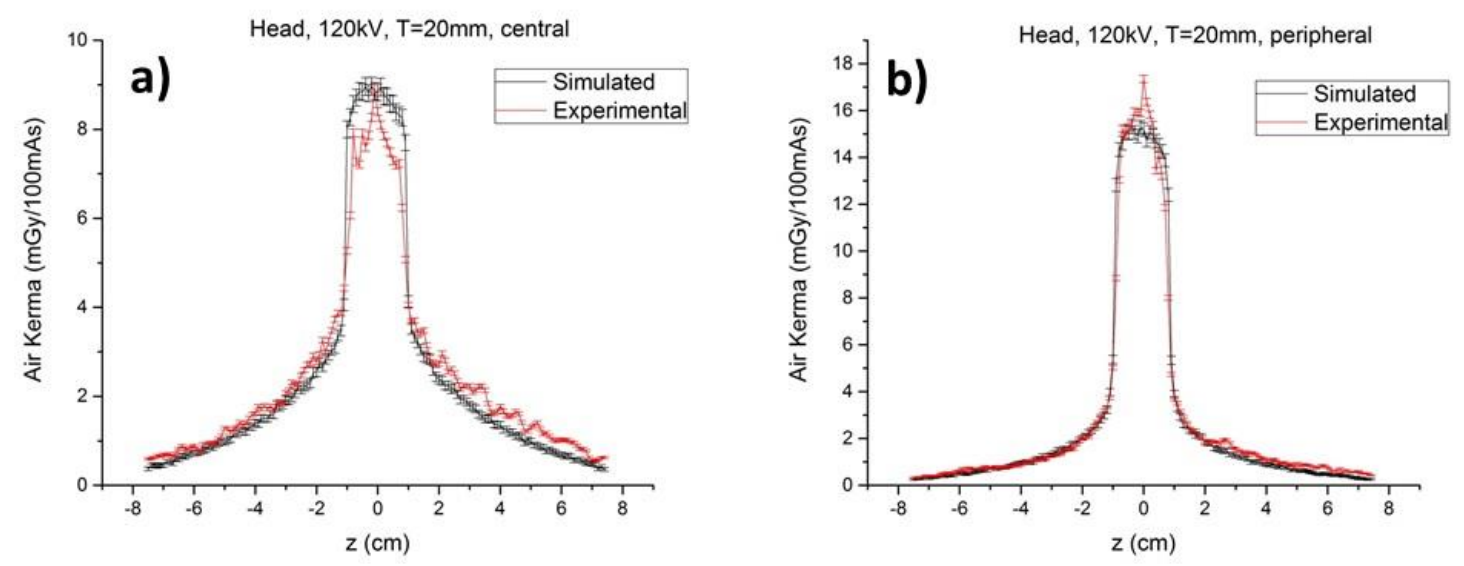

Figure 56. a) Central and b) Peripheral Dose Profile in Head CTDI phantom at $120 \mathrm{kV}$ and $\mathbf{n T}=\mathbf{2 0 m m}$ beam width. Comparison between simulated (black line) and experimental (red line) Dose Profiles.
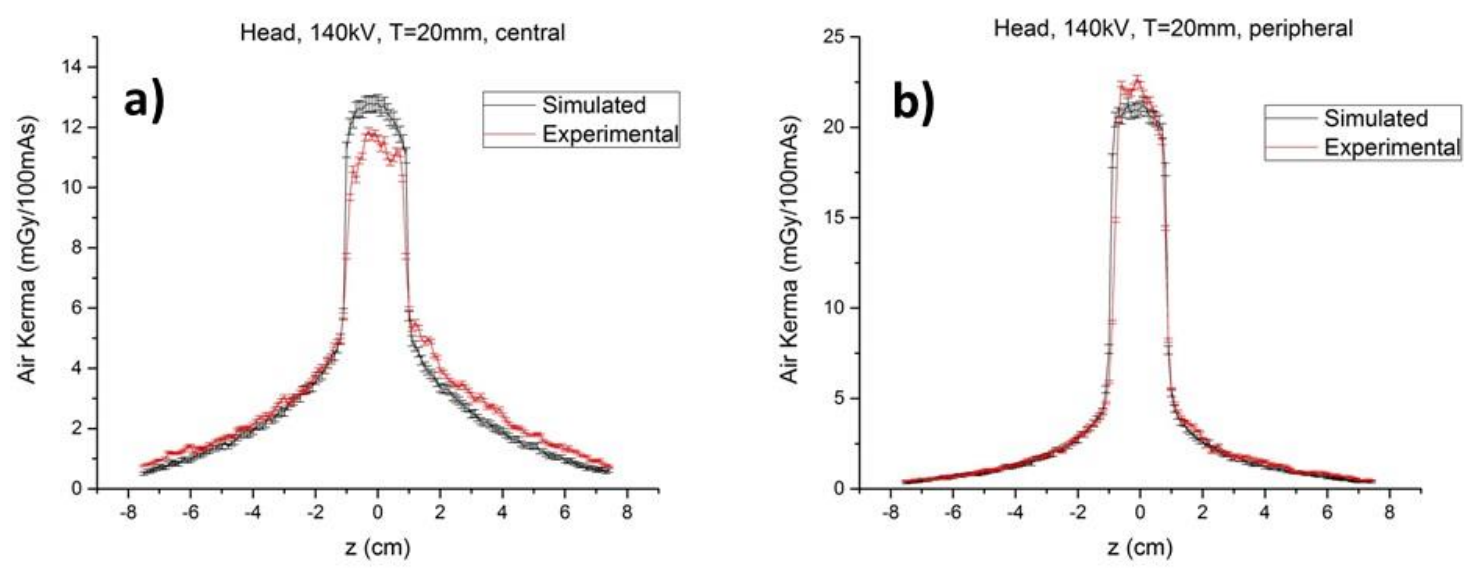

Figure 57. a) Central and b) Peripheral Dose Profile in Head CTDI phantom at $140 \mathrm{kV}$ and nT=20mm beam width. Comparison between simulated (black line) and experimental (red line) Dose Profiles. 

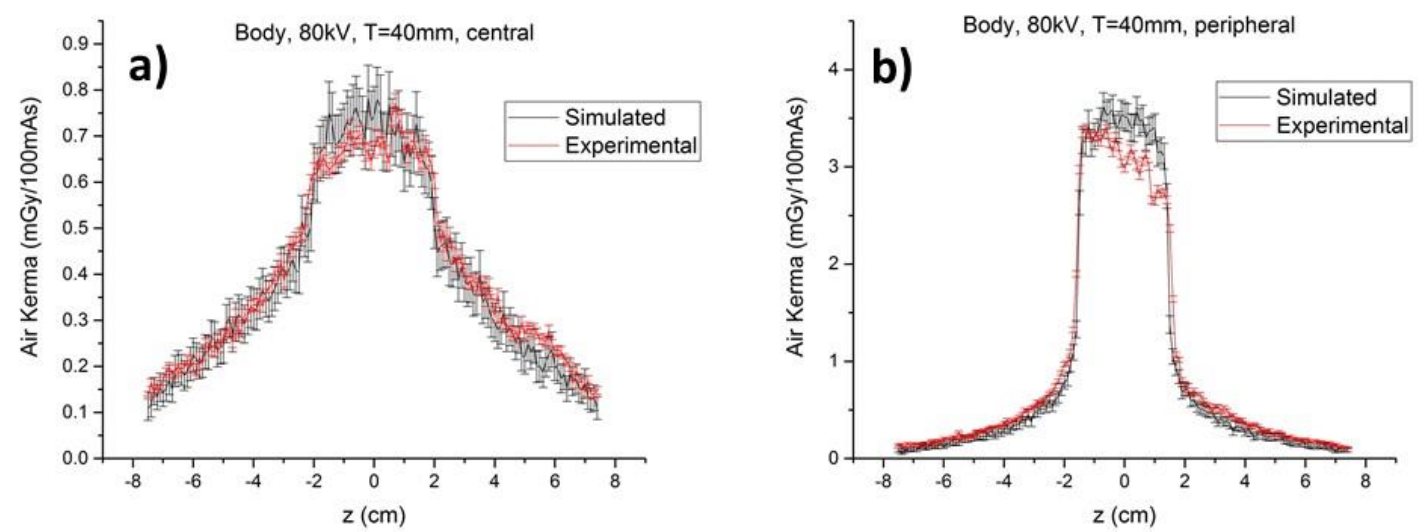

Figure 58. a) Central and b) Peripheral Dose Profile in Body CTDI phantom at $80 \mathrm{kV}$ and $\mathrm{nT}=40 \mathrm{~mm}$ beam width. Comparison between simulated (black line) and experimental (red line) Dose Profiles.
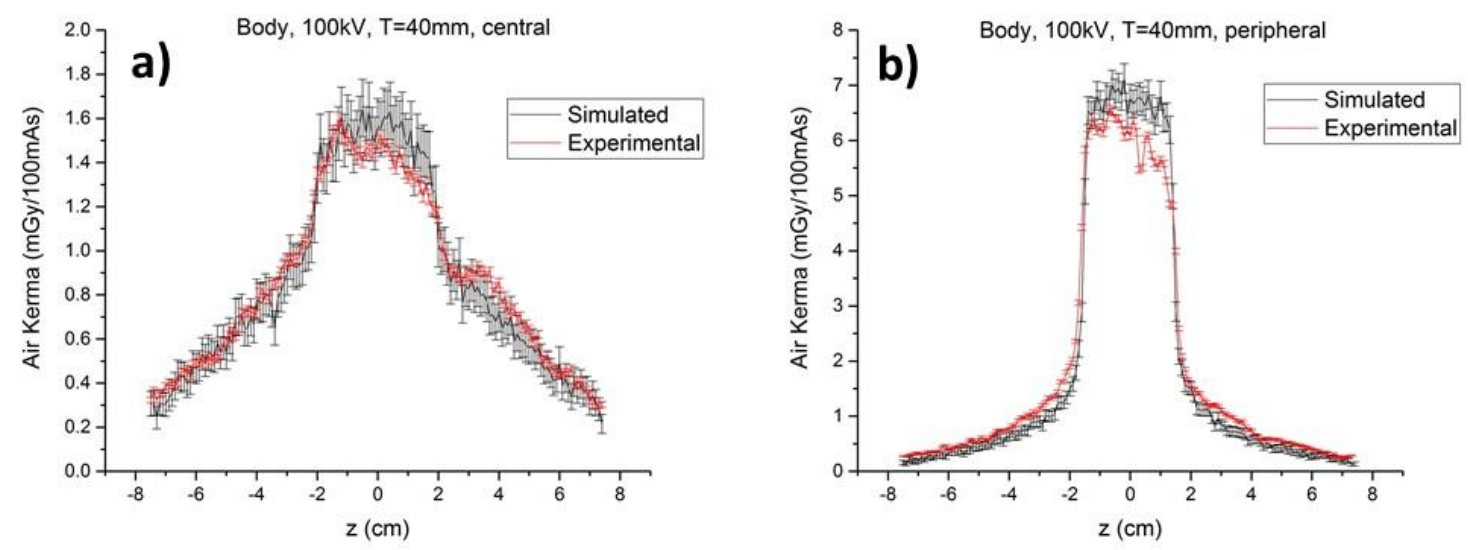

Figure 59. a) Central and b) Peripheral Dose Profile in Body CTDI phantom at $100 \mathrm{kV}$ and nT $=40 \mathrm{~mm}$ beam width. Comparison between simulated (black line) and experimental (red line) Dose Profiles.
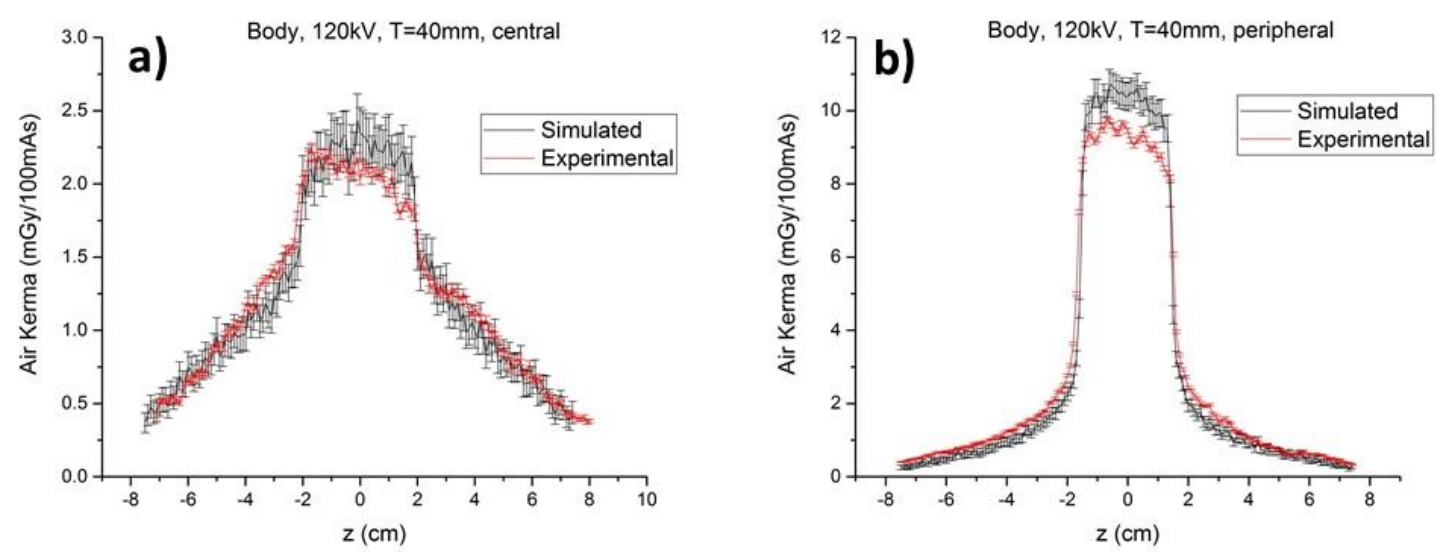

Figure 60. a) Central and b) Peripheral Dose Profile in Body CTDI phantom at $120 \mathrm{kV}$ and $\mathrm{nT}=40 \mathrm{~mm}$ beam width. Comparison between simulated (black line) and experimental (red line) Dose Profiles. 

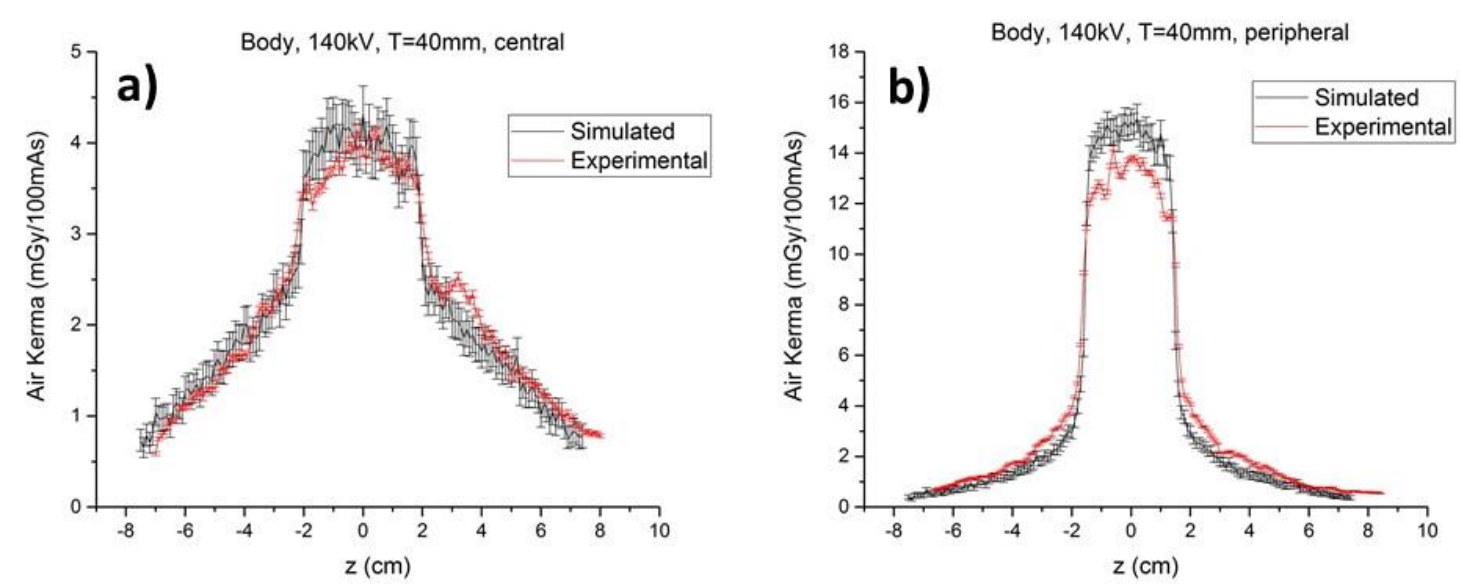

Figure 61. a) Central and b) Peripheral Dose Profile in Body CTDI phantom at $140 \mathrm{kV}$ and nT=40mm beam width. Comparison between simulated (black line) and experimental (red line) Dose Profiles.

\subsection{Cumulated Dose Profiles for Helical/Sequential Protocols}

Currently the Helical/Sequential CT acquisitions are the most common procedures in CT (see, Section 2.1.3 for details of CT acquisition modes). They are composed by a number of X-ray tube rotations along the patient, number which depend on the length of the imaged volume. The increasing number of CT examinations deserves the tracking of the radiation exposure delivered in a CT procedure in order to reduce the radiation risk of the patients. An accurate method of characterization of the radiation exposure (or dose) delivered by a helical/sequential CT procedure is the assessment of the cumulated dose profile that is a representative indicator of the deposited dose distribution in a CTDI phantom. In this Section, the cumulative dose profiles computed from MC simulation in head and body CTDI phantoms along their central and peripheral axes are presented. Previous works developed analytical (Dixon et al., 2005, Dixon and Boone, 2011), experimental (Tsai et al., 2003, Mori et al., 2005) and even MC simulation (Zhang et al., 2009) procedures to assess the cumulated dose profiles from Helical/Sequential CT examination in CTDI phantoms for some specific cases. This work shows a comprehensive study of the cumulated dose profiles for Helical CT procedures aiming to compute the currently proposed CT dose metrics reported in the AAPM report 111 (Dixon and Ballard, 2007, Dixon, 2019, AAPM, 2010).

Before presenting the simulated cumulative profiles, a set of experimental measurements of the cumulated dose profiles for sequential and helical protocols in CTDI phantoms are analyzed in order to have them as references for discussion and verification of conformity of the cumulated dose profiles computed from MC simulation. 


\subsubsection{Cumulated Dose Profiles from experimental measurements}

The experimental procedure followed to measure the cumulated dose profiles for Helical/Sequential protocols are described in Section 3.3.2. The CT scanner where the measurements were carried out was the GE Discovery CT750 HD.

\subsubsection{Sequential Cumulated Dose Profiles}

Figure 62 presents cumulated dose profiles measured along the central and peripheral axes of the head CTDI phantom using OSL ribbons. Scan parameters were set as, $n T=10 \mathrm{~mm}$, $I=10 \mathrm{~mm}$, in Figure 62.a, and $I=15 \mathrm{~mm}$, in Figure 62.b. In this sequential mode, $I$ is the table displacement between single axial irradiation (or distance between the midpoint to midpoint of contiguous fan beams on the AOR). Cumulative profiles in Figure 62 correspond to four Xray tube rotations. The cumulative-profile shapes depend on the setting of the acquisition parameters, for instance, in Figure 62.a, peaks in the cumulative profiles in both the central and peripheral position appeared. The peaks suggests superposition of single axial dose profiles for both central and peripheral positions, in contrast, in Figure 62.b, regions with no direct irradiation of the fan beam are presented (since, $I>n T$ ) in the cumulative dose profiles as regions in which the profile fall, as "valleys".
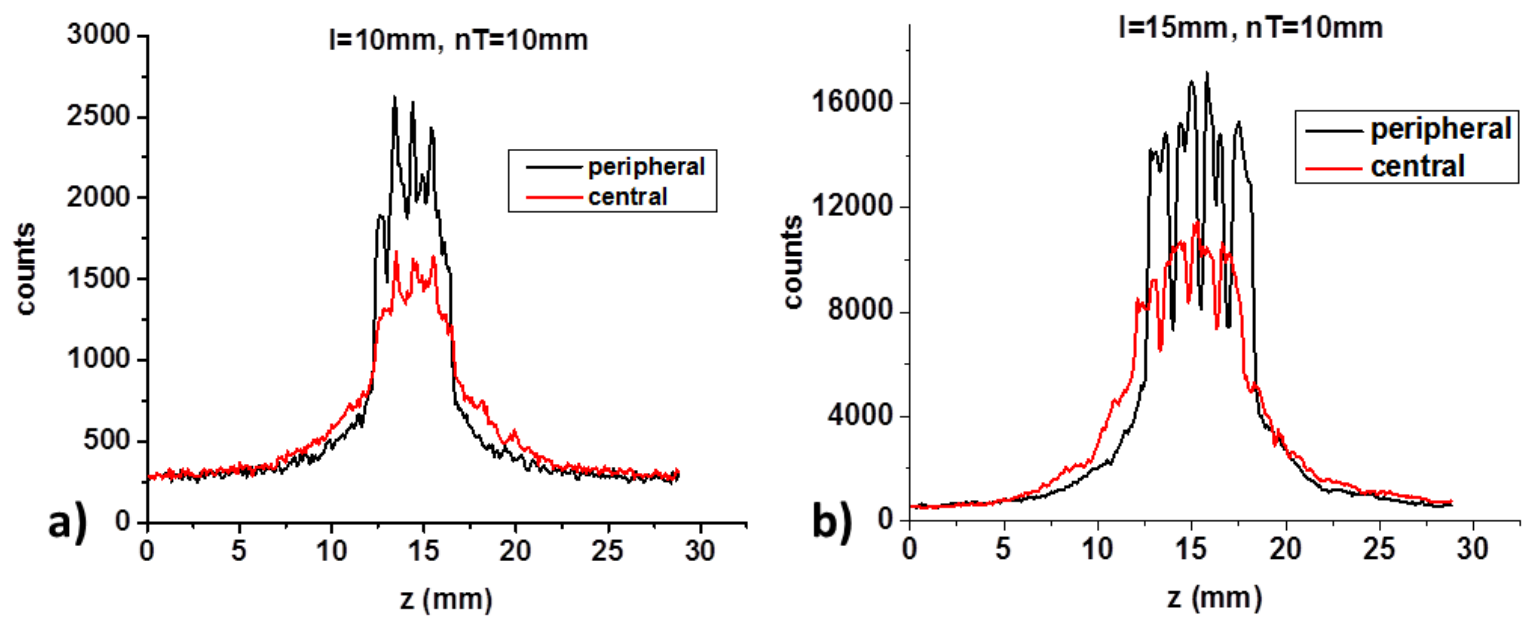

Figure 62. Sequential cumulative dose profiles along the central and peripheral axes of the head CTDI phantom. The imaging parameter is $n T=10 \mathrm{~mm}$ in both cases and a) $I=10 \mathrm{~mm}$ and b) $I=15 \mathrm{~mm}$. Measured using OSL ribbons. Measurements for $120 \mathrm{kV}$.

\subsubsection{Helical Cumulated Dose Profiles}

Figure 63.a presents cumulated dose profiles measured along a central and three peripheral positions of a CTDI body phantom for helical scanning. Scan parameters were, pitch=1.375 and $n T=40 \mathrm{~mm}$. In Figure 63.a, the cumulated dose profile along the peripheral axes present "valleys" that are a result of the pitch value $(p>1)$ and the attenuation of the CT 
table since the CTDI phantom were located on the CT table for the measurements. For a helical scanning with $p>1$, there are regions in the CTDI phantom that are not irradiated directly (see, section 2.1.3), this fact in addition with the CT table attenuation shapes the cumulative profile. The influence of the CT table attenuation on the cumulative profile along the central axis appears to be smoothed by the attenuation of the phantom material. Figure 63.b compares cumulative profiles one along the central axis and other along the peripheral axis of a CTDI body phantom for three pitch values, $0.531,0.696$, and 1.375 and two nominal beam widths, $n T=20 \mathrm{~mm}$ and $n T=40 \mathrm{~mm}$. The influences of the CT-table attenuation and the pitch values appear to be smoothing in the cumulative dose profile along the central axis. In the case of the peripheral cumulative profile for pitch, $p=0.531$, the pitch values conform the cumulative profile as a sequence of peaks and valleys. Figure 64 shows central and peripheral cumulative profiles, separately.
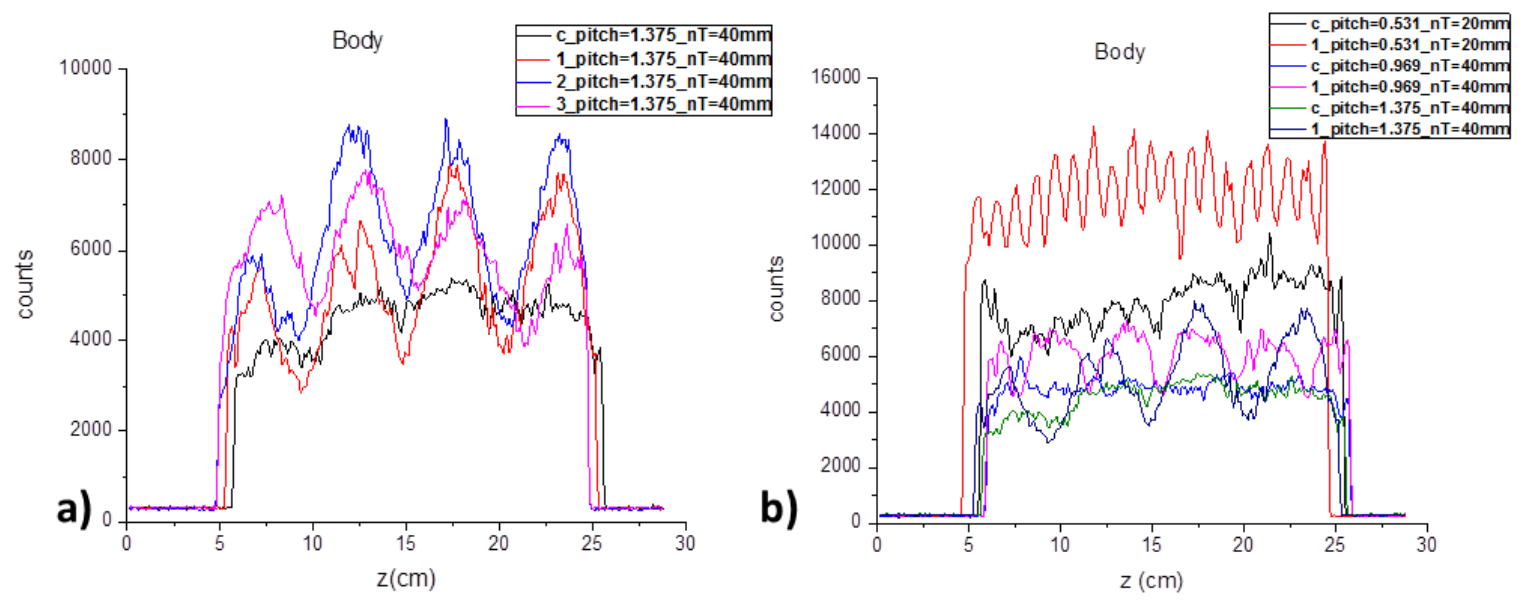

Figure 63. Accumulative dose profiles in CTDI body phantom at central and peripheral positions a) pitch=1.375 and $n T=40 \mathrm{~mm}$ and b) pitch values: $0.531,0.969$, and 1.375 and $n T=20 \mathrm{~mm}$ and $n T=40 \mathrm{~mm}$. Measured using OSL ribbons. Measurements for $120 \mathrm{kV}$.
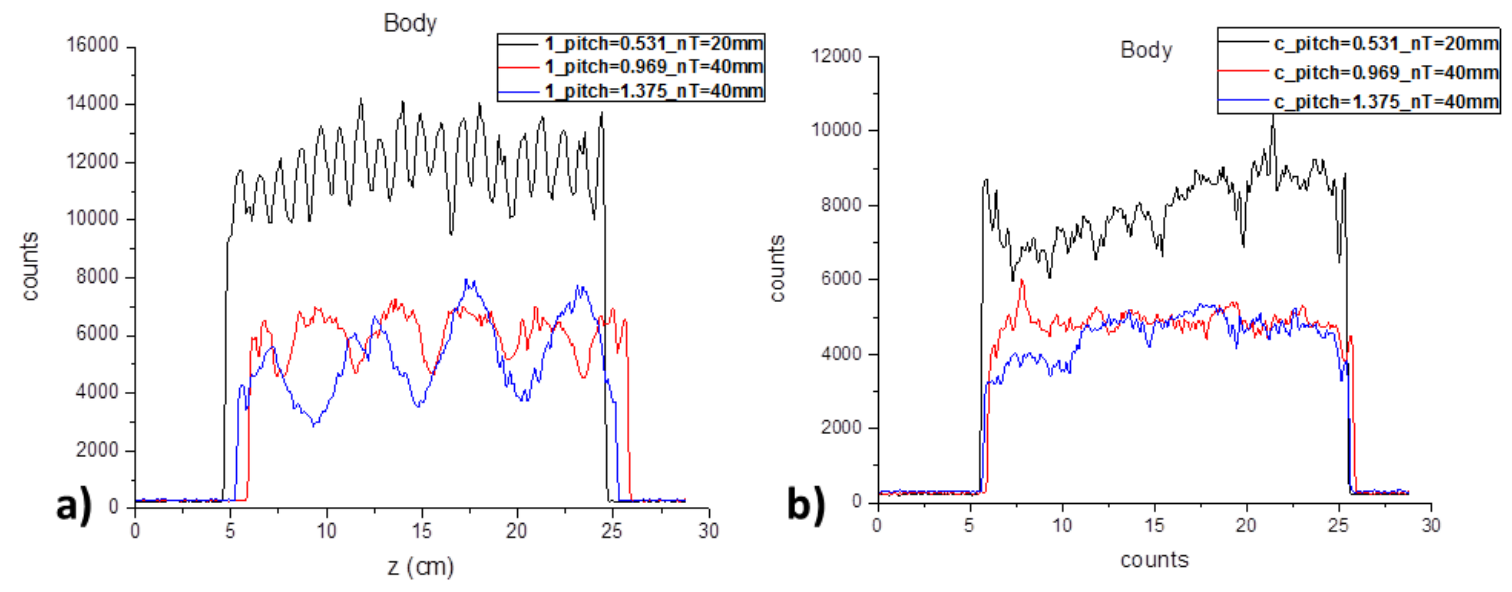
Figure 64. Accumulative dose profiles in CTDI body phantom for pitch values:0.531, 0.969, and 1.375, imaged volume length of $n T=20 \mathrm{~mm}$ and $n T=40 \mathrm{~mm}$, along the a) central and b) peripheral positions. Measured using OSL ribbons.

\subsubsection{Cumulated Dose Profiles from MC simulation}

Following the head and body clinical protocols presented in Table 6, cumulated dose profiles were simulated in the standard CTDI phantoms. In helical scan mode, the patient table is translated during the $\mathrm{CT}$ examination, comprising the $\mathrm{X}$-ray tube rotation. This CT helical mode was implemented doing that the photon emission of X-ray source model simulate the rotation and translation at the same time, maintaining the CTDI phantom static. The MC implementation of the helical mode is explained in Section 3.1.2, considering that the patient table attenuation was not implemented. Figure 65.a and Figure 65.b illustrates helical cumulative dose profiles computed along the central and peripheral axes of the head CTDI phantom for pitches values of $0.531,0.969$, and 1.375 , beam width, $b=20 \mathrm{~mm}$, and $120 \mathrm{kV}$ X-ray spectrum. These dose profiles correspond to one rotation and they are in term of air kerma with units of (mGy/100mAs). Also in Figure 65.a and Figure 65.b, are presented the central and peripheral dose profile for single axial irradiation (X-ray source is maintained static) just for reference and comparison with the helical profiles.
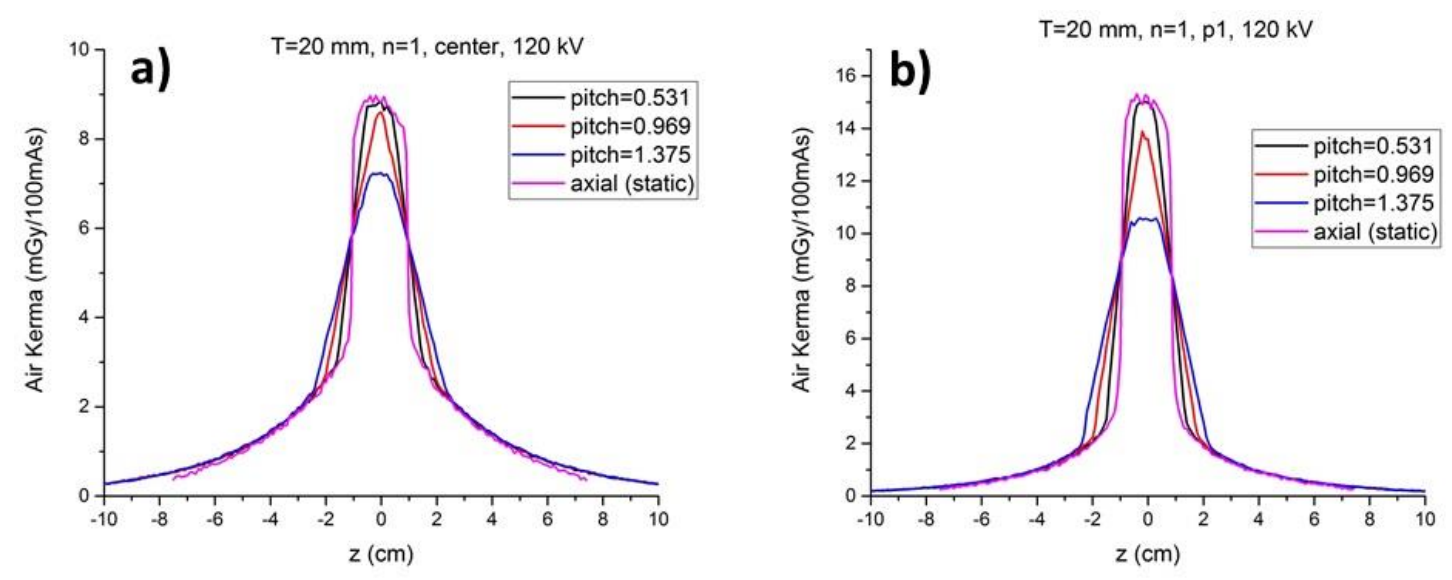

Figure 65. a) Central and b) Peripheral Dose profile for $120 \mathrm{kV}$ in term of air kerma (mGy/100 mAs) for pitch values: $0.531,0.969$ and 1.375 for head helical protocol. Both helical and axial dose profile correspond to a single rotation $(n=1)$ with beam width $20 \mathrm{~mm}$.

Figure 66.a and Figure 66.a illustrate helical cumulative dose profiles computed along the central and peripheral axes of the CTDI body phantom for pitches values of $0.516,0.984$, and 1.375 , beam width, $b=40 \mathrm{~mm}$, and $120 \mathrm{kV} \mathrm{X}$-ray spectrum. These dose profiles correspond to one rotation and they are in term of air kerma with units of (mGy/100mAs). Also in Figure 65.a and Figure 65.b, are presented the central and peripheral dose profile for 
single axial irradiation (X-ray source is maintained static) just for reference and comparison with the helical profiles.
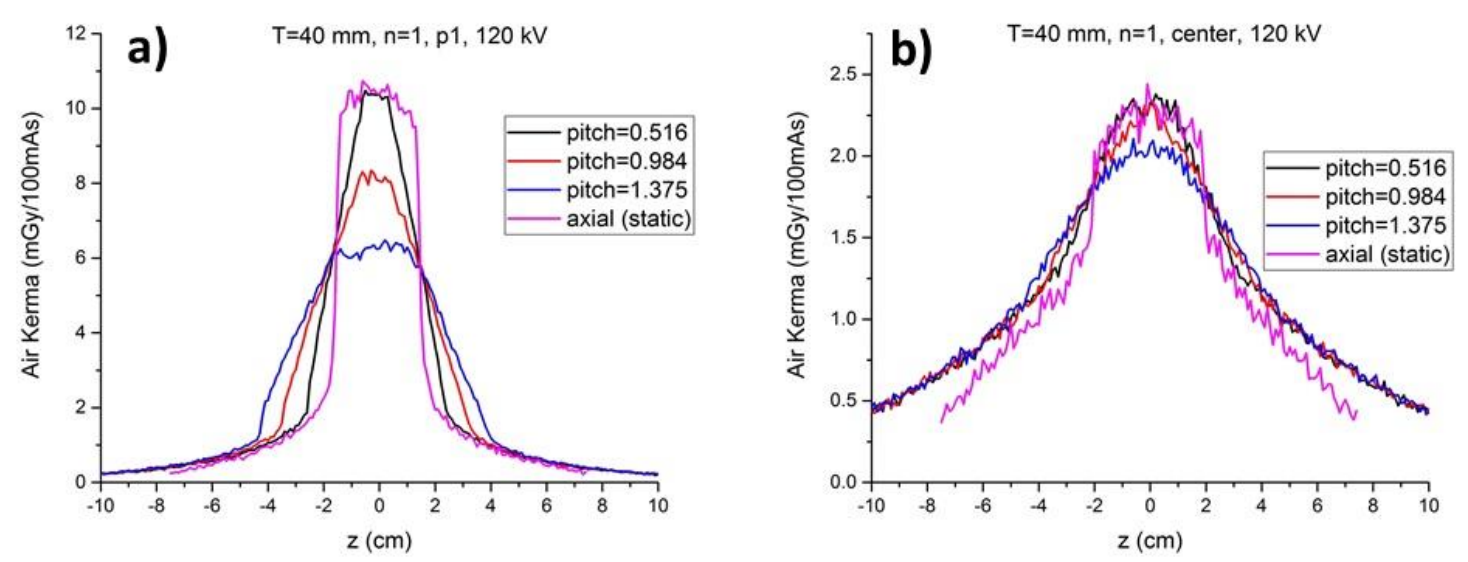

Figure 66. a) Peripheral and b) Central Dose profile for $120 \mathrm{kV}$ in term of air kerma (mGy/100 mAs) for pitch values: $0.516,0.984$ and 1.375 for body helical protocol. Both helical and axial dose profile correspond to a single rotation $(n=1)$ with beam width $40 \mathrm{~mm}$.

Appendix C registers all cumulated dose profile for head (pitch values of $0.531,0.969$ and 1.375) and body (pitch values of $0.516,0.984$ and 1.375 ) at $120 \mathrm{kV}$. The cumulated dose profiles were simulated up to reach their equilibrium value at $\mathrm{z}=0$, as illustrated in each case. All cumulated dose profiles are calibrated in terms of air kerma with the conversion coefficients presented in Table 12 and Table 13.

Figure 67.a and Figure 67.b present cumulative dose profiles computed along the central peripheral axes of the head CTDI phantom for ten rotation of the X-ray source, pitch values of $0.531,0.969$, and 1.375 and for $120 \mathrm{kV} \mathrm{X-ray} \mathrm{spectrum.} \mathrm{The} \mathrm{deposited} \mathrm{energy} \mathrm{in} \mathrm{the}$ CTDI phantom do not depend of the pitch value, but, depend of the number of rotations (fixing the other parameters). This fact can be verified from Figure 67 in which the integration of central dose profile (or peripheral dose profile) may be result in the same value independently of the pitch value. For lower pitch values the deposited energy is more concentrated. In contrast, for high pitch values the deposited energy is broader. The same statement is visualized in Figure 68, in which for the CTDI body phantom, beam width $\mathrm{b}=40 \mathrm{~mm}$ and $120 \mathrm{kV} \mathrm{X}$-ray spectrum, the deposited dose is more concentrated for lower pitch values than for higher. Note that, for $\mathrm{p}=1.375$, valleys are identified in the peripheral profile for both head and body protocols, this issue can be understood due to the displacement of the patient table for one rotation of the X-ray source is major than the beam width. Therefore, there are region that are not directly irradiated for the beam. The valley in central 
cumulative profiles do not appeared because they are smoothed by the phantom material attenuation.
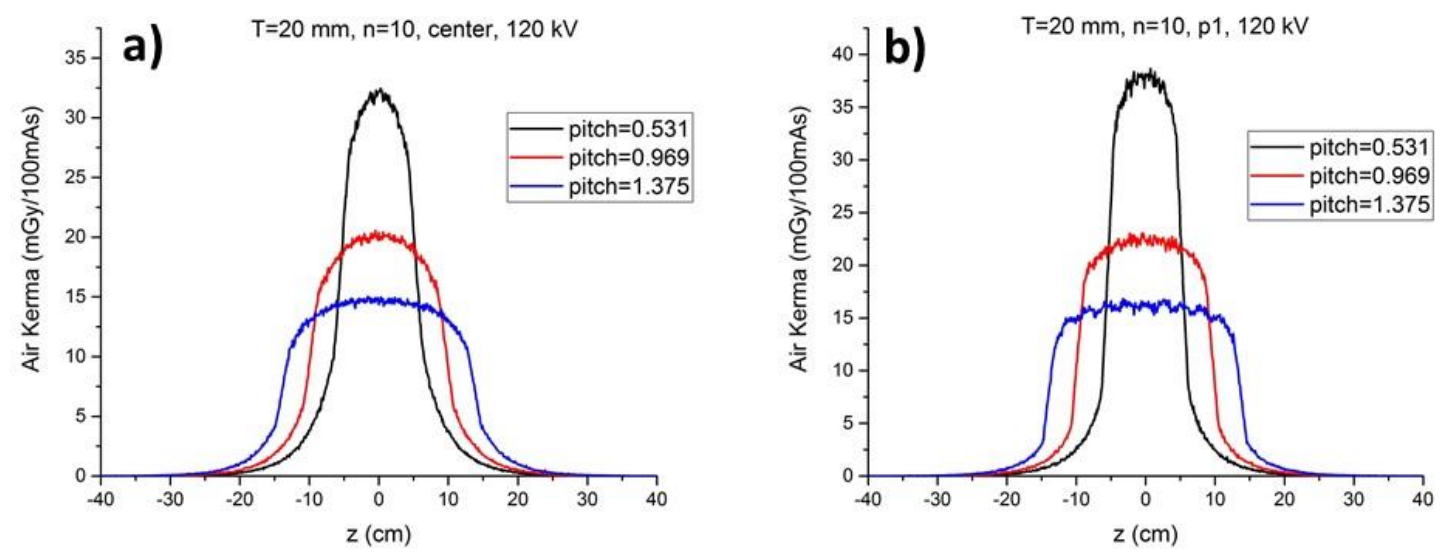

Figure 67. a) Central and b) Peripheral Dose profile for $120 \mathrm{kV}$ in term of air Kerma (mG/100 mAs) for pitch values: $0.531,0.969$ and 1.375 for head helical protocol. Both helical and axial dose profile correspond to a 10 rotations $(n=10)$ with beam width $20 \mathrm{~mm}$.
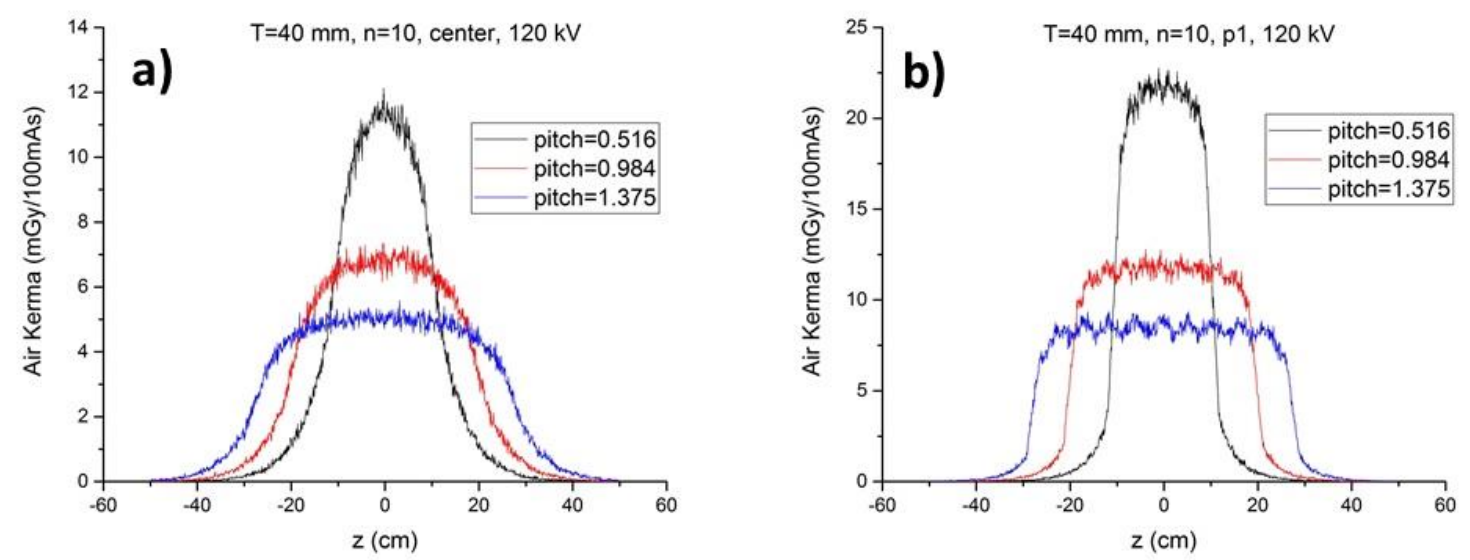

Figure 68. a) Central and b) Peripheral Dose profile for $120 \mathrm{kV}$ in term of air Kerma (mG/100 mAs) for pitch values: $\mathbf{0 . 5 1 6}, \mathbf{0 . 9 8 4}$ and 1.375 for body helical protocol. Both helical and axial dose profile correspond to a 10 rotations $(n=10)$ with beam width $40 \mathrm{~mm}$.

Figure 69 and Figure 70 show a comparison of the cumulated dose profiles for one and ten rotation of the X-ray source. It is instructive to see, that a cumulated dose profile for $n>1$, is obtained by superposition of the dose profile corresponding to a single rotation, shown in each case. 

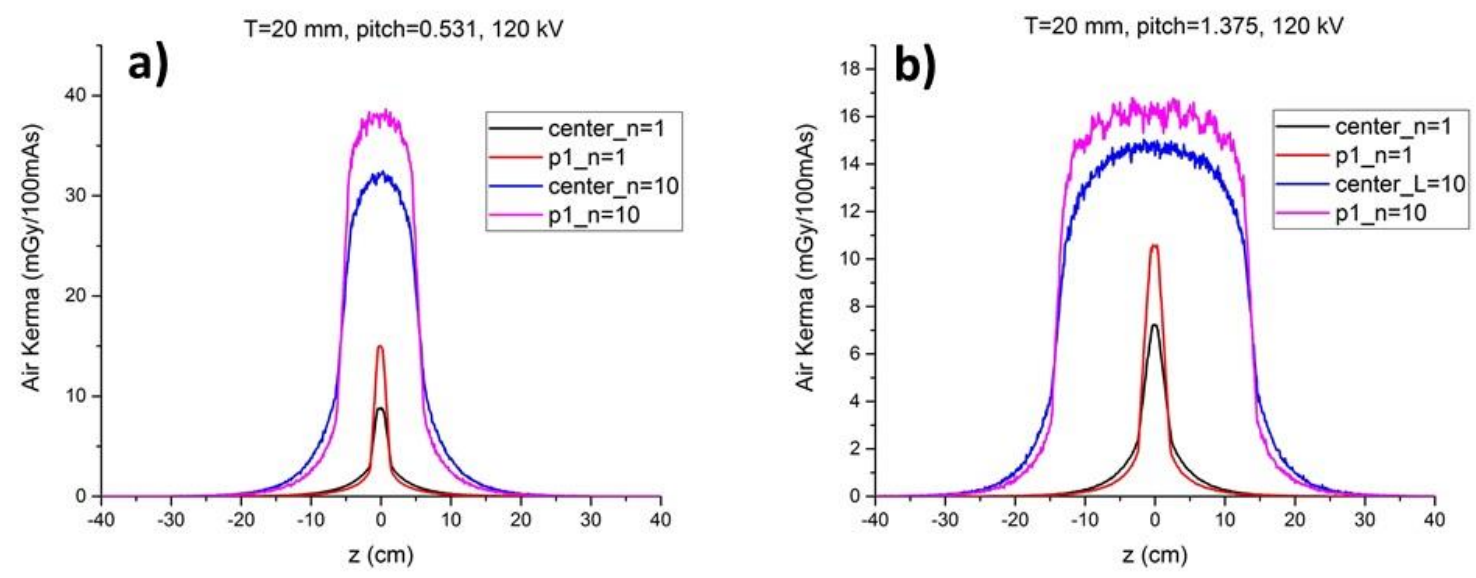

Figure 69. a) Dose profiles at central and peripheral axes for 0.531 pitch value and 1 and 10 rotations, b) Dose profiles at central and peripheral axes for 1.375 pitch value and 1 and 10 rotations. With $120 \mathrm{kV}$ and $20 \mathrm{~mm}$ beam width.
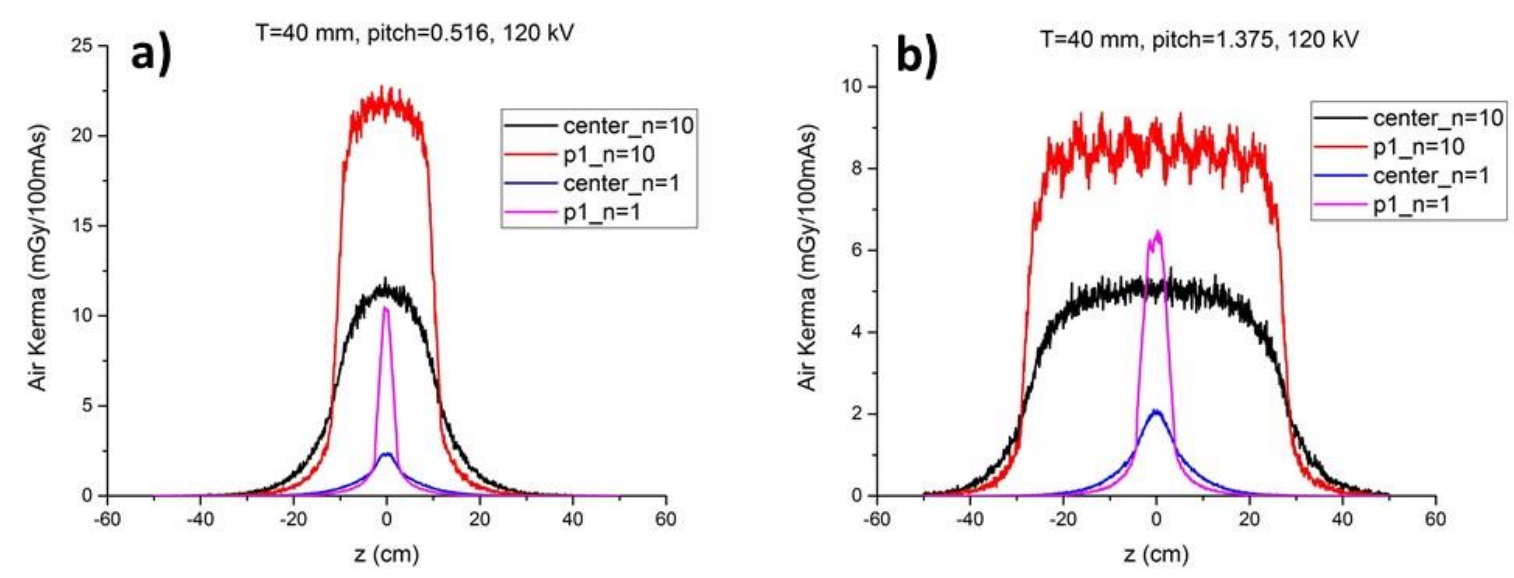

Figure 70. a) Dose profiles at central and peripheral axes for 0.516 pitch value and 1 and 10 rotations, b) Dose profiles at central and peripheral axes for 1.375 pitch value and 1 and 10 rotations. With $120 \mathrm{kV}$ and $40 \mathrm{~mm}$ beam width.

Figure 71 and Figure 72 show a set of cumulative dose profiles computed from MC simulation along the central and peripheral axes of the CTDI head and body phantoms, respectively. The figures show profiles from one to ten X-ray source rotations, for pitch $1.375,120 \mathrm{kV}$ X-ray spectrum, beam width of $20 \mathrm{~mm}$ (head phantom) and $40 \mathrm{~mm}$ (body phantom). 

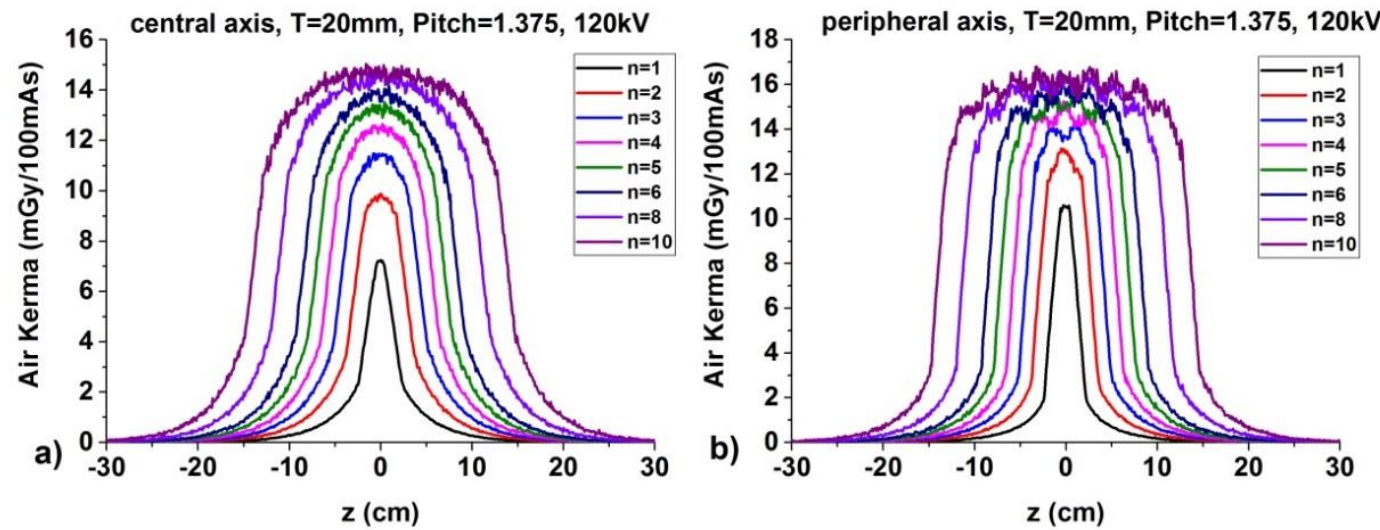

Figure 71. a) Central and b) peripheral cumulated dose profile in head CTDI phantom for helical scan with pitch=1.375.
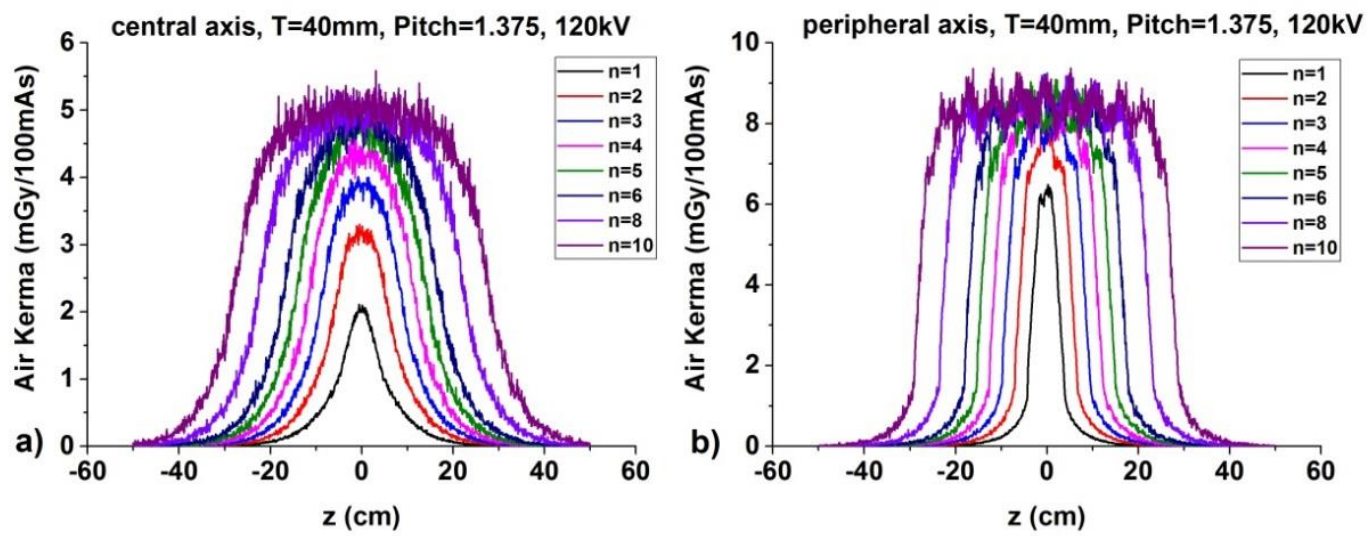

Figure 72. a) Central and b) peripheral cumulated dose profile in body CTDI phantom for helical scan with pitch=1.375.

\subsection{Novel metrics for CT Dosimetry}

Novel metrics for CT dosimetry can be derived from the cumulative dose profiles computed from MC simulation calibrated in terms of air kerma per $100 \mathrm{mAs}$ (mGy/100 mAs). Dose Line Integral $(D L I)$, Equilibrium Dose $\left(D_{e q}\right)$, the product Pitch x Equilibrium Dose $\left(p x D_{e q}\right)$, and the Approach to Equilibrium function $(H(L))$ can be derived from the cumulative dose profiles. Also, the Equilibrium Scanning Length $\left(L_{e q}\right)$ can be assessed by fitting the curve of $H(L)$ with the model shown in Equation (63).

Figure 73 and Figure 74 illustrate the plot $D L I$ versus $L$ for pitch values of $0.531,0.75$, $0.969,1.375$, and 1.5 for the head helical protocol, and pitch values of $0.516,0.75,0.984$, 1.375 , and 1.5 for the body helical protocol at $120 \mathrm{kV}$ X-ray spectrum. The nominal beam width onto the AOR were $20 \mathrm{~mm}$ and $40 \mathrm{~mm}$ for the head and body protocols, respectively. Figure 73.a and Figure 74.a present the curve $D L I$ versus $L$ for the central and peripheral axes 
of the head and body CTDI phantoms, respectively. Figure 73.b and Figure 74.b present the curve weighted-DLI $\left(D L I_{w}\right)$ versus $L$ for the head and body CTDI phantoms, respectively. $D L I_{w}$ was computed weighting the central-DLI $\left(D L I_{c}\right)$ and peripheral- $D L I\left(D L I_{p}\right)$, with the coefficients $1 / 3$ and $2 / 3$, respectively, according to Equation (59). It is important to note that the DLI units are (mGy/100mAs).cm which is a result of the dimensional analysis in Equation (58) and it has the same unit of the Dose Length Product, DLP, which is a quantity derived from the $C T D I_{100}$. The error bars shown for each $D L I$ value were computed by the corresponding propagation of the uncertainty of each point in the corresponding calibrated cumulative dose profile with scan length, $L$. As derived from the analytical formulation of Dixon et al, $D L I$ is proportional to $L$, with the $D_{e q}$ as the constant of proportionality, as shown in Equation (42). For each curve in Figure 73 and Figure 74, a linear model was used (fixing the independent term to zero) to estimate the angular coefficient of the model which will be the estimative of $D_{e q}$. The Linear Curve Fitting tool of the OriginPro 8.6 software (OriginLab Corporation, Northampton, MA, USA) was used to fit the linear model to each curve. In all cases, the R-Square of the fitting was 1, which denotes data with good correlation.

Appendix D present all curves of the $D L I$ versus $L$ computed in this work for pitch values of $0.531,0.969$, and 1.375 , for the head helical protocol, nominal beam width of $n T=20 \mathrm{~mm}$, and pitch values of $0.516,0.984$, and 1.375 for the body helical protocol, beam width of $n T=40 \mathrm{~mm}$, at $80 \mathrm{kV}, 100 \mathrm{kV}, 120 \mathrm{kV}$ (additionally pitch 0.75 and 1.5) and $140 \mathrm{kV}$ $\mathrm{X}$-ray spectra.

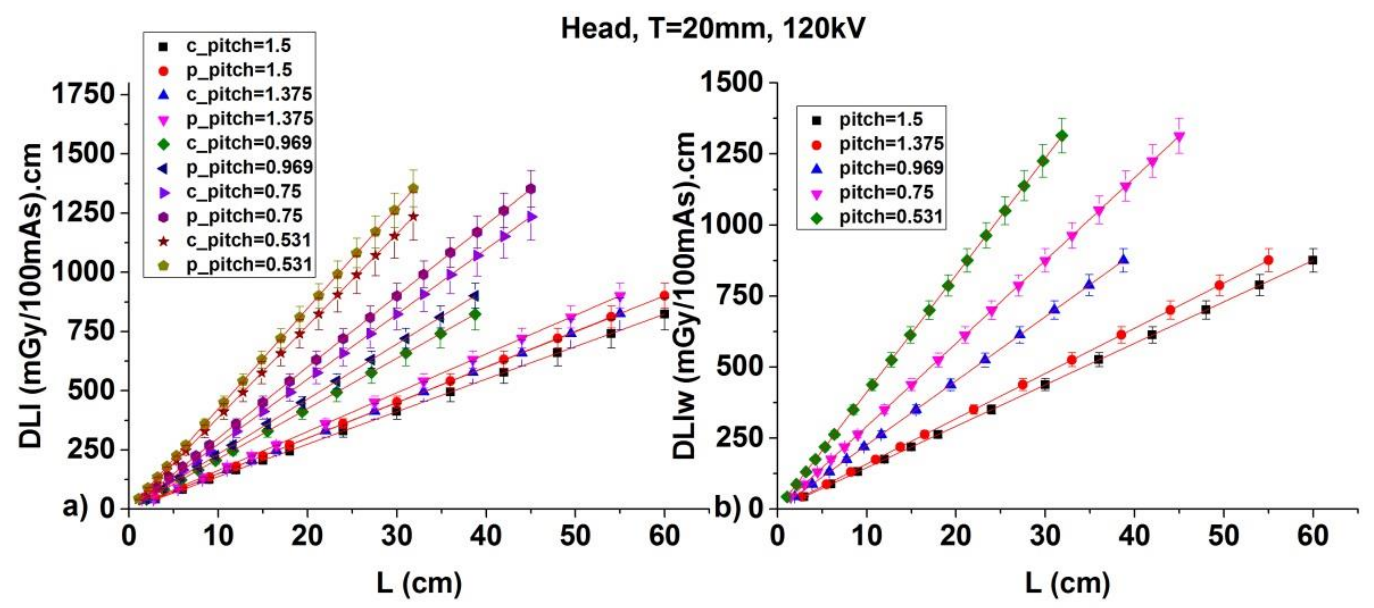

Figure 73. Simulated Dose Line Integral for head helical protocols of pitch values: 1.5, 1.375, 0.969, 0.75 and 0.531 at $120 \mathrm{kV}$ as a function of the scan length, $L$, a) along the central $\left(D L I_{c}\right)$ and peripheral $\left(D L I_{p}\right)$ axes and for b) Weighted-DLI, $D L I_{w}$. Simulations were performed in an infinite cylindrical phantom of PMMA with $16 \mathrm{~cm}$ diameter and beam width of $20 \mathrm{~mm}$. 

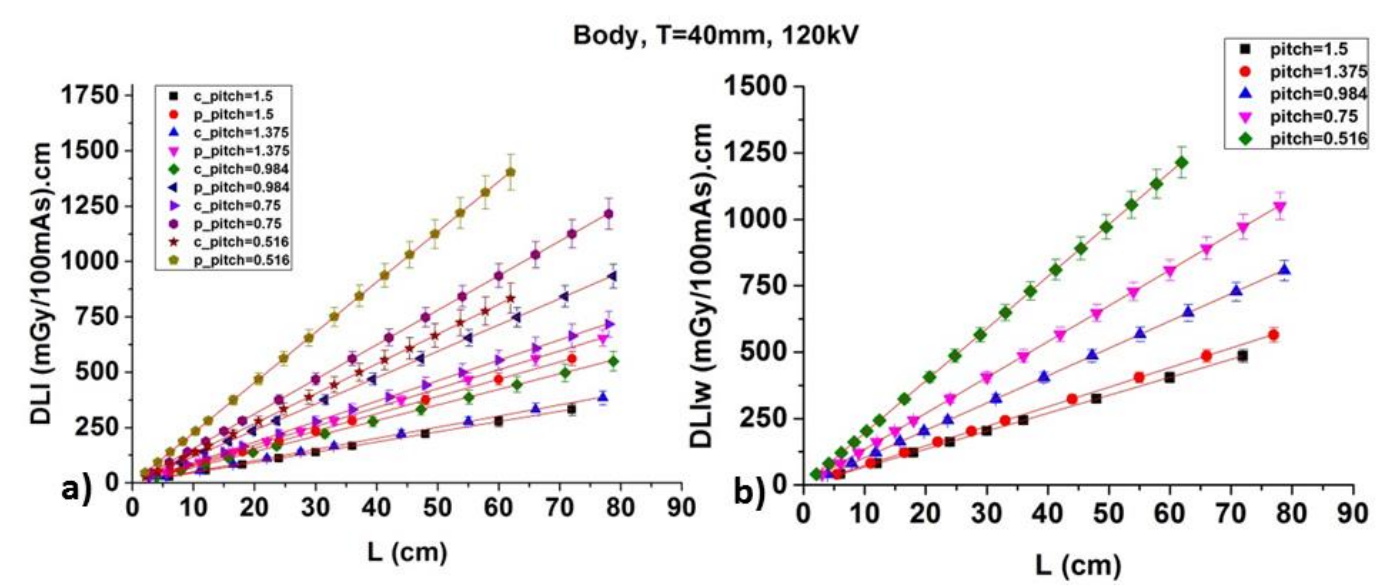

Figure 74. Simulated Dose Line Integral for body helical protocols of pitch values: 1.5, 1.375, 0.984, 0.75 and 0.516 at $120 \mathrm{kV}$ as a function of the irradiation length, $L$, a) along the central $\left(D L I_{c}\right)$ and peripheral

$\left(D L I_{p}\right)$ axes and for b) Weighted-DLI, $D L I_{w}$. Simulations were performed in an infinite cylindrical phantom of PMMA with $32 \mathrm{~cm}$ diameter and beam width of $40 \mathrm{~mm}$.

As mentioned above, an estimative of $D_{e q}$ may be obtained by fitting the data set in the Graph DLI vS $L$ with a linear model, in which the intercept must be fixed to 0, as suggested in Equation (42). Table 14 and Table 15 present the central- $D_{e q}, D_{e q, c}$, and peripheral- $D_{e q}, D_{e q, p}$, related to the corresponding axes of the head and body CTDI phantoms for helical irradiation, as well as, the weighted- $D_{e q}, D_{e q, w}$, which was computed fitting the curve $D L I_{w}$ versus $L$. For X-ray spectra corresponding to $80,100,120$, and $140 \mathrm{kV}$. Pitch values for the head protocol were $0.531,0.969$, and 1.375 , for the body protocols were 0.516 , 0.984, and 1.375. Additionally, pitch values of 1.5 and 0.75 in the case of $120 \mathrm{kV}$ were computed for both head and body protocols. The data comes from fitting each linear curve presented in Appendix D. As is indicated in Table 14 and Table 15, the units of the $D_{e q}$ in all cases is $\mathrm{mGy} / 100 \mathrm{mAs}$.

Table 14. Equilibrium Dose, $D_{e q}$ as a function of typical pitch values for Head helical protocols $(a=20 \mathrm{~mm}) . D_{e q}$ was obtained by fitting the DLI using a linear model where the intercept was fixed in 0.

The R-Square of the fitting was 1 for all cases.

\begin{tabular}{|c|c|c|c|c|}
\hline \multicolumn{5}{|c|}{$D_{e q}(\mathrm{mGy} / 100 \mathrm{mAs})$} \\
\hline Voltage & Pitch & $D_{e q, c}$ & $D_{e q, p}$ & $D_{e q, w}$ \\
\hline \multirow{3}{*}{$80 \mathrm{kV}$} & 0.531 & $12.842(3)$ & $15.730(3)$ & $14.767(2)$ \\
\hline & 0.969 & $7.028(5)$ & $8.611(4)$ & $8.083(4)$ \\
\hline & 1.375 & $4.951(5)$ & $6.066(4)$ & $5.694(4)$ \\
\hline \multirow{3}{*}{$100 k V$} & 0.531 & $24.643(5)$ & $27.859(4)$ & $26.787(4)$ \\
\hline & 0.969 & $13.48(1)$ & $15.254(8)$ & $14.664(8)$ \\
\hline & 1.375 & $8.368(8)$ & $9.491(7)$ & $9.117(7)$ \\
\hline \multirow{4}{*}{$120 \mathrm{kV}$} & 0.531 & $38.773(8)$ & $42.426(7)$ & $41.208(7)$ \\
\hline & 0.75 & $27.455(5)$ & $30.048(6)$ & $29.184(5)$ \\
\hline & 0.969 & $21.249(4)$ & $23.253(3)$ & $22.585(3)$ \\
\hline & 1.375 & $14.983(4)$ & $16.388(2)$ & $15.919(2)$ \\
\hline
\end{tabular}




\begin{tabular}{lcccc} 
& $\mathbf{1 . 5}$ & $13.731(2)$ & $15.025(3)$ & $14.594(2)$ \\
\hline & $\mathbf{0 . 5 3 1}$ & $55.29(1)$ & $59.06(1)$ & $57.80(1)$ \\
$\mathbf{1 4 0 k V}$ & $\mathbf{0 . 9 6 9}$ & $30.26(3)$ & $32.33(2)$ & $31.64(2)$ \\
& $\mathbf{1 . 3 7 5}$ & $21.30(3)$ & $22.79(2)$ & $22.29(2)$ \\
\hline
\end{tabular}

Table 15. Equilibrium Dose, $D_{e q}$ as a function of typical pitch values for Body helical protocols $(a=20 \mathrm{~mm}) . D_{e q}$ was obtained by fitting the DLI using a linear model where the intercept was fixed in 0 . The R-Square of the fitting was 1 for all cases.

\begin{tabular}{lcccc}
\hline \multicolumn{5}{c}{$\boldsymbol{D}_{\text {eq }}(\mathbf{m G y} / \mathbf{1 0 0 m A s})$} \\
\hline Voltage & Pitch & $\boldsymbol{D}_{\text {eq, }}$ & $\boldsymbol{D}_{\text {eq, } \boldsymbol{p}}$ & $\boldsymbol{D}_{\text {eq, } \boldsymbol{w}}$ \\
\hline & $\mathbf{0 . 5 1 6}$ & $4.040(3)$ & $7.304(3)$ & $6.216(2)$ \\
$\mathbf{8 0 k V}$ & $\mathbf{0 . 9 8 4}$ & $2.120(1)$ & $3.830(1)$ & $3.260(1)$ \\
& $\mathbf{1 . 3 7 5}$ & $1.516(1)$ & $2.743(1)$ & $2.334(1)$ \\
\hline & $\mathbf{0 . 5 1 6}$ & $9.127(4)$ & $14.694(4)$ & $12.839(3)$ \\
$\mathbf{1 0 0 k V}$ & $\mathbf{0 . 9 8 4}$ & $4.785(2)$ & $7.712(2)$ & $6.737(2)$ \\
& $\mathbf{1 . 3 7 5}$ & $3.420(2)$ & $5.518(2)$ & $4.819(2)$ \\
\hline & $\mathbf{0 . 5 1 6}$ & $13.449(6)$ & $22.715(6)$ & $19.626(4)$ \\
& $\mathbf{0 . 7 5}$ & $9.239(4)$ & $15.621(5)$ & $13.494(4)$ \\
$\mathbf{1 2 0 k V}$ & $\mathbf{0 . 9 8 4}$ & $7.047(7)$ & $11.905(4)$ & $10.286(4)$ \\
& $\mathbf{1 . 3 7 5}$ & $5.036(7)$ & $8.521(2)$ & $7.360(4)$ \\
& $\mathbf{1 . 5}$ & $4.623(3)$ & $7.811(3)$ & $6.748(3)$ \\
\hline & $\mathbf{0 . 5 1 6}$ & $24.20(1)$ & $32.47(1)$ & $29.714(8)$ \\
$\mathbf{1 4 0 k V}$ & $\mathbf{0 . 9 8 4}$ & $12.683(6)$ & $17.022(5)$ & $15.575(3)$ \\
& $\mathbf{1 . 3 7 5}$ & $9.072(7)$ & $12.181(4)$ & $11.145(3)$ \\
\hline
\end{tabular}

The $D_{e q}$ presented in Table 14 and Table 15 are normalized by the tube current and irradiation time product, but, $D_{e q}$ has dependence with others scanning parameters such as the $\mathrm{X}$-ray spectrum, nominal beam width, and pitch values. To put in evidence the dependence of $D_{e q}$ with the X-ray spectrum, it is useful to compute the product between the pitch value and $D_{e q}, p x D_{e q}$. Table 16 and Table 17 present $p x D_{e q}$ product (data obtained from Table 14 and Table 15) for the head and body helical protocols for nominal beam width of $20 \mathrm{~mm}$ and $40 \mathrm{~mm}$, respectively. It is interesting to note that $p x D_{e q}$ has no significant variation with the pitch value, less than $0.3 \%$ for all cases in both head and body helical protocols. It is evident 
the dependence with X-ray spectrum for the four tube voltage for both head and body Helical protocols.

Table 16. Pitch value $x$ Equilibrium Dose, $p x D_{e q}$ as a function of typical pitch values for Head helical protocols $(n T=20 \mathrm{~mm})$.

\begin{tabular}{lcccc}
\hline \multicolumn{5}{c}{$\boldsymbol{p} \boldsymbol{x} \boldsymbol{D}_{\boldsymbol{e q}}(\mathbf{m G y} / \mathbf{1 0 0 m A s})$} \\
\hline Voltage & Pitch & $\boldsymbol{p} \boldsymbol{x} \boldsymbol{D}_{\boldsymbol{e q}, \boldsymbol{c}}$ & $\boldsymbol{p} \boldsymbol{x} \boldsymbol{D}_{\boldsymbol{e q}, \boldsymbol{p}}$ & $\boldsymbol{p} \boldsymbol{x} \boldsymbol{D}_{\boldsymbol{e q}, \boldsymbol{w}}$ \\
\hline \multirow{3}{*}{ 80kV } & $\mathbf{0 . 5 3 1}$ & $6.819(2)$ & $8.353(2)$ & $7.841(1)$ \\
& $\mathbf{0 . 9 6 9}$ & $6.810(5)$ & $8.344(4)$ & $7.832(4)$ \\
& $\mathbf{1 . 3 7 5}$ & $6.808(7)$ & $8.341(6)$ & $7.829(6)$ \\
\hline \multirow{2}{*}{$\mathbf{1 0 0 k V}$} & $\mathbf{0 . 5 3 1}$ & $13.085(3)$ & $14.793(2)$ & $14.224(2)$ \\
& $\mathbf{0 . 9 6 9}$ & $13.062(10)$ & $14.781(8)$ & $14.209(8)$ \\
& $\mathbf{1 . 3 7 5}$ & $11.506(11)$ & $13.050(10)$ & $12.536(10)$ \\
\hline \multirow{2}{*}{$\mathbf{0 . 5 3 1}$} & $20.588(4)$ & $22.528(4)$ & $21.881(4)$ \\
& $\mathbf{0 . 7 5}$ & $20.591(4)$ & $22.536(5)$ & $21.888(4)$ \\
& $\mathbf{0 . 9 6 9}$ & $20.590(4)$ & $22.532(3)$ & $21.885(3)$ \\
& $\mathbf{1 . 3 7 5}$ & $20.602(6)$ & $22.534(3)$ & $21.889(3)$ \\
& $\mathbf{1 . 5}$ & $20.597(3)$ & $22.538(5)$ & $21.891(3)$ \\
\hline \multirow{3}{*}{ 140kV } & $\mathbf{0 . 5 3 1}$ & $29.359(5)$ & $31.361(5)$ & $30.692(5)$ \\
& $\mathbf{0 . 9 6 9}$ & $29.322(29)$ & $31.328(19)$ & $30.659(19)$ \\
& $\mathbf{1 . 3 7 5}$ & $29.288(41)$ & $31.336(28)$ & $30.649(28)$ \\
\hline
\end{tabular}

Table 17. Pitch value $x$ Equilibrium Dose, $p x D_{e q}$ as a function of typical pitch values for Body helical protocols $(n T=40 \mathrm{~mm})$.

\begin{tabular}{lcccc}
\hline \multicolumn{5}{c}{$\boldsymbol{p} \boldsymbol{x} \boldsymbol{D}_{\boldsymbol{e q}}(\mathbf{m G y} / \mathbf{1 0 0 m A s})$} \\
\hline Voltage & Pitch & $\boldsymbol{p} \boldsymbol{x} \boldsymbol{D}_{\text {eq, } \boldsymbol{c}}$ & $\boldsymbol{p} \boldsymbol{x} \boldsymbol{D}_{\text {eq }, \boldsymbol{p}}$ & $\boldsymbol{p} \boldsymbol{x} \boldsymbol{D}_{\text {eq, } \boldsymbol{w}}$ \\
\hline \multirow{3}{*}{$\mathbf{8 0 k V}$} & $\mathbf{0 . 5 1 6}$ & $2.085(2)$ & $3.769(2)$ & $3.207(1)$ \\
& $\mathbf{0 . 9 8 4}$ & $2.086(1)$ & $3.769(1)$ & $3.208(1)$ \\
& $\mathbf{1 . 3 7 5}$ & $2.085(1)$ & $3.772(1)$ & $3.209(1)$ \\
\hline & $\mathbf{0 . 5 1 6}$ & $4.710(2)$ & $7.582(2)$ & $6.625(2)$ \\
$\mathbf{1 0 0 k V}$ & $\mathbf{0 . 9 8 4}$ & $4.708(2)$ & $7.589(2)$ & $6.629(2)$ \\
& $\mathbf{1 . 3 7 5}$ & $4.703(3)$ & $7.587(3)$ & $6.626(3)$ \\
\hline & $\mathbf{0 . 5 1 6}$ & $6.940(3)$ & $11.721(3)$ & $10.127(2)$ \\
& $\mathbf{0 . 7 5}$ & $6.929(3)$ & $11.716(4)$ & $10.121(3)$ \\
$\mathbf{1 2 0 k V}$ & $\mathbf{0 . 9 8 4}$ & $6.934(7)$ & $11.715(4)$ & $10.121(4)$ \\
& $\mathbf{1 . 3 7 5}$ & $6.925(10)$ & $11.716(3)$ & $10.120(6)$ \\
& $\mathbf{1 . 5}$ & $6.935(5)$ & $11.717(5)$ & $10.122(5)$ \\
\hline
\end{tabular}




\begin{tabular}{lllll}
\hline & $\mathbf{0 . 5 1 6}$ & $12.487(5)$ & $16.755(5)$ & $15.332(4)$ \\
$\mathbf{1 4 0 k V}$ & $\mathbf{0 . 9 8 4}$ & $12.480(6)$ & $16.750(5)$ & $15.326(3)$ \\
& $\mathbf{1 . 3 7 5}$ & $12.474(10)$ & $16.749(6)$ & $15.324(4)$ \\
\hline
\end{tabular}

As defined in Section 2.1.3, the pitch $(p)$, defined for helical scanning, quantifies the longitudinal overlap $(p<1)$, gap $(p>1)$, or contiguity $(p=1)$ between successive groups of tomographic sections. It is defined as, $p=b / n T$, where $b$ is the table advance per rotation ( $b=v \tau$ ) during helical scanning. For $n$ reconstructed tomographic sections, each of nominal width $T, n T$ is the total nominal beam width (along the AOR) associated with the group of sections simultaneously acquired and yielding multiple images associated with a single scan. Since the equilibrium dose, $D_{e q}$ is proportional to the ratio $a / b, D_{e q}(a, b) \propto a / b$, then, the product $p x D_{e q}(a, b) \propto a / n T$, and its proportionality term, $a / n T$, that is the over-beaming factor (Dixon and Ballard, 2007). The over-beaming factor for the GE Discovery CT750 HD can be obtained from the primary profiles in Figure 47 and Figure 48, where $a$ is the FWHM at $z=0$ of the primary profile and $n T$ is the nominal beam width. It is instructive to compute the mean values for $p x D_{e q}$ from Table 16 and Table 17 to have representative values as a function of the tube voltages, aiming to be used in acceptance tests to evaluate dose at any values of the clinical pitches. Table 18 presents the mean values of the product $p x D_{e q}$ for Head $(n T=20 \mathrm{~mm})$ and Body $(n T=40 \mathrm{~mm})$ helical protocols and Figure 75 presents the data from Table 18.

Table 18. Mean value of Pitch value $x$ Equilibrium Dose, $p x D_{e q}$ as a function of typical pitch values for Head $(n T=20 \mathrm{~mm})$ and Body $(n T=40 \mathrm{~mm})$ helical protocols.

\begin{tabular}{ccccccc}
\hline \multicolumn{8}{c}{ Mean $\boldsymbol{p} \boldsymbol{x} \boldsymbol{D}_{\boldsymbol{e q}}(\mathbf{m G y} / \mathbf{1 0 0 m A s})$} \\
\hline Head $(\boldsymbol{n T} \boldsymbol{T}=\mathbf{2 0} \mathbf{~ m m})$ & \multicolumn{3}{c}{ Body $(\boldsymbol{n T} \boldsymbol{T}=\mathbf{4 0} \mathbf{~ m m})$} \\
\hline $\mathbf{~ V o l t a g e}$ & $\boldsymbol{p} \boldsymbol{x} \boldsymbol{D}_{\boldsymbol{e q}, \boldsymbol{c}}$ & $\boldsymbol{p} \boldsymbol{x} \boldsymbol{D}_{\boldsymbol{e q}, \boldsymbol{p}}$ & $\boldsymbol{p} \boldsymbol{x} \boldsymbol{D}_{\boldsymbol{e q}, \boldsymbol{w}}$ & $\boldsymbol{p} \boldsymbol{x} \boldsymbol{D}_{\boldsymbol{e q}, \boldsymbol{c}}$ & $\boldsymbol{p} \boldsymbol{x} \boldsymbol{D}_{\boldsymbol{e q}, \boldsymbol{p}}$ & $\boldsymbol{p} \boldsymbol{x} \boldsymbol{D}_{\boldsymbol{e q}, \boldsymbol{w}}$ \\
\hline $\mathbf{1 0 0 k V}$ & $6.812(9)$ & $8.346(7)$ & $7.834(7)$ & $2.085(2)$ & $3.770(2)$ & $3.208(2)$ \\
$\mathbf{1 2 0 k V}$ & $13.074(10)$ & $14.787(8)$ & $14.217(8)$ & 4.707() 4 & $7.586(4)$ & $6.627(4)$ \\
$\mathbf{1 4 0 k V}$ & $20.594(9)$ & $22.533(8)$ & $21.887(7)$ & $6.932(13)$ & $11.717(8)$ & $10.122(9)$ \\
& $29.32(5)$ & $31.34(3)$ & $30.667(3)$ & $12.480(12)$ & $16.751(9)$ & $15.328(7)$ \\
\hline
\end{tabular}




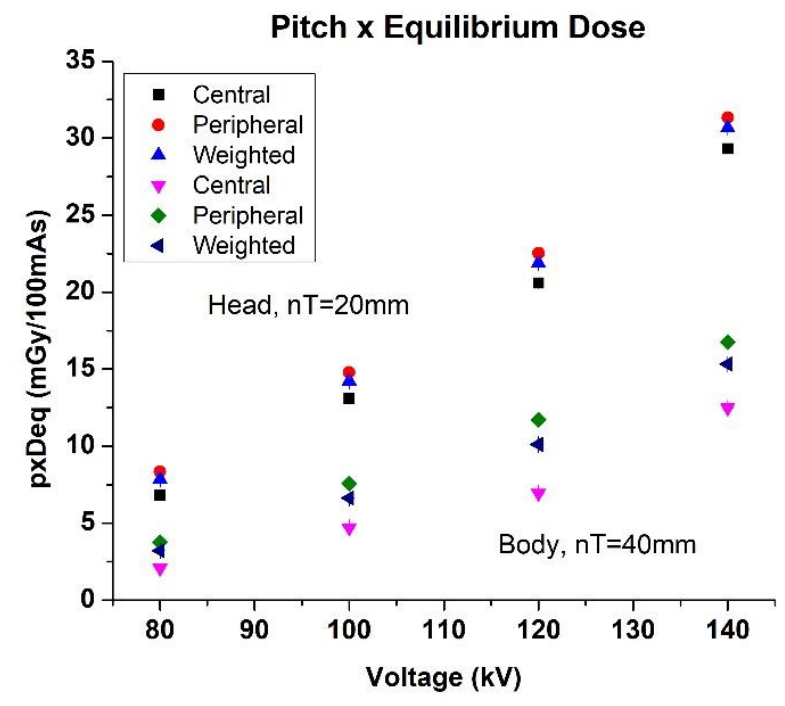

Figure 75. Pitch $x$ Equilibrium Dose product, $p x D_{e q}$, for both head and Helical protocols at $n T=20 \mathrm{~mm}$ and $\boldsymbol{n T}=\mathbf{4 0} \mathrm{mm}$, respectively. Data is presented for the central and peripheral axis, and also the weighted $D_{e q}$ is presented, for both head and body helical protocols. The graph show clearly the dependence of the $p x D_{e q}$ with the tube voltage.

As was stated in Section $2.4 .2, D_{e q}$ is the asymptotic value of the cumulative dose profile at $\mathrm{z}=0, D_{L}(0)$, (or dose at the midpoint of the cumulative profile) which is reached at sufficiently large scanning length, $L$. Figure 76 shows how the dose at $z=0$ from the cumulated profile at the central (Figure 76.a) and peripheral (Figure 76.b) axes reach an asymptotic value when the number of X-ray source's rotations increases for the particular case of head CTDI phantom, $n T=20 \mathrm{~mm}$, pitch value of 1.375 and $120 \mathrm{kV} \mathrm{X-ray} \mathrm{spectrum.}$ The number of X-ray source's rotations, $N$, is directly related to the scanning length by $L=N b$.
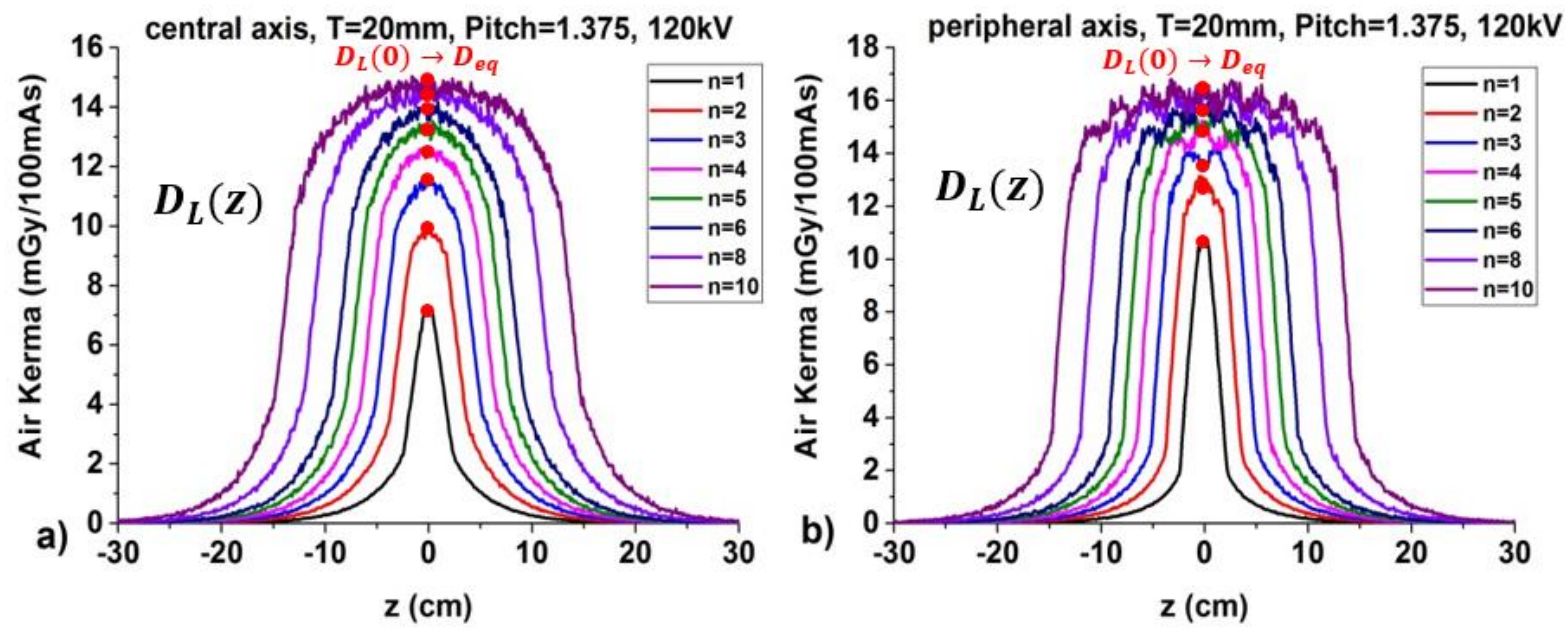

Figure 76. Cumulated dose profiles, $D_{L}(z)$, of CT Helical examinations for a number of X-ray source rotations, from 1 to 10 , along the a) central and b) peripheral axes of the head CTDI phantom. This data 
were obtained by Monte Carlo simulation with scan parameters, $n T=20 \mathrm{~mm}$, pitch=1.375, and $120 \mathrm{kV}$ Xray spectrum.

Another approach to estimate the Equilibrium Dose, $D_{e q}$, can be obtained from the analysis of the $D_{L}(0)$ versus $L$ curve, in which $D_{L}(0)$ tends to its asymptotic value as $\mathrm{L}$ increase. Figure 77.a shows simulated $D_{L}(0)$ for helical scanning simulation at the central and peripheral axes of the head CTDI phantom for pitch values of $0.531,0.969$, and 1.375, $n T=20 \mathrm{~mm}$ and $80 \mathrm{kV}$ X-ray spectrum. As can be visualized in Figure 77.a, $D_{L}(0)$ reaches an equilibrium from certain value of $L, D_{L}(0)$ is higher at the central axis than at the peripheral one for the same pitch in all cases. Figure 77.b shows the weighted- $D_{L}(0)$ computed from center- $D_{L}(0)$ and peripheral- $D_{L}(0)$ in Figure 77.a for each pitch values. It is possible to visualize from Figure 77 that $D_{L}(0)$ is higher for low pitch than for a high pitch. It make sense, since low pitch values imply in a shorter volume length irradiated than for high pitch for the same rotation time, therefore, the same energy is absorbed in largest volume for high pitch than for low pitch.
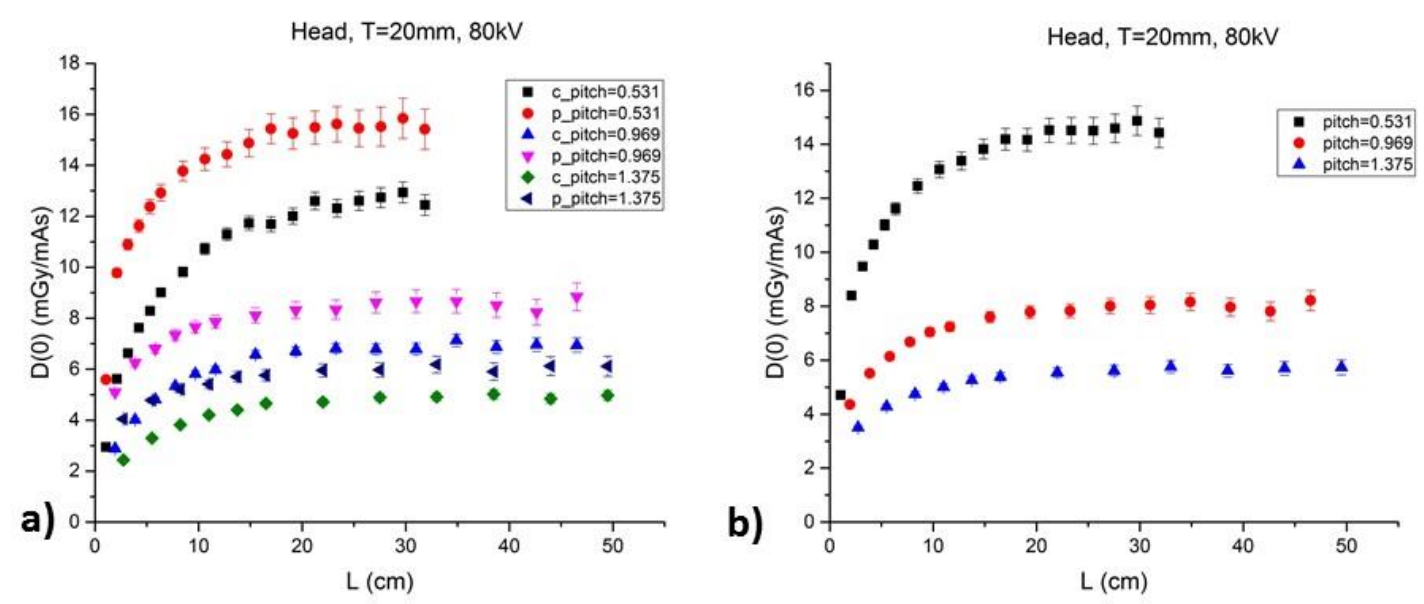

Figure 77. Maximum dose at $\mathrm{z}=0$ of the cumulated dose profile, $D_{L}(0)$, from a helical scanning simulation at $80 \mathrm{kV}$ X-ray spectrum, a) $D_{L}(0)$ at the central and peripheral axes of the head CTDI phantom, and b) weighted- $D_{L}(0)$ computed from $D_{L}(0)$ at central and peripheral axes.

An interesting way to show how $D_{L}(0)$ increase up to reach a plateau is presented in Figure $78,79,80$, and 81 , where each value of $D_{L}(0)$ is divided by its corresponding asymptotic value or $D_{e q}$ registered in Table 14 for the case of the head CTDI phantom with $n T=20 \mathrm{~mm}$. The quotient $D_{L}(0) / D_{e q}$ becomes independent of the CT technique factors, such as, tube current, rotation time, and pitch, as can be seen in the figures, there are clearly two set of points corresponding to $D_{L}(0) / D_{e q}$ at central and peripheral axes of the head CTDI 
phantom. This behavior can be seen as well for the weighted- $D_{L}(0)$ computed from to $D_{L}(0) / D_{e q}$ at central and peripheral axes of the head CTDI phantom. Figure $82,83,84,85$ correspond to $D_{L}(0) / D_{e q}$ at central and peripheral axes of the body CTDI phantom for $n T=40 \mathrm{~mm}$. Data for $D_{L}(0) / D_{e q}$ were obtained for $80,100,120$ and $140 \mathrm{kV}, \mathrm{X}$-ray spectra and the pitch values were $0.531,0.969$ and 1.375 for head and $0.516,0.984$ and 1.375 for body protocols. The curve $D_{L}(0) / D_{e q}$ versus $L$ is the so-called approach-to-equilibrium function, $H(L)$, (Dixon and Boone, 2010, AAPM, 2010). $H(L)$ represents a practical method to compute $D_{L}(0)$ for an arbitrary $L$ since the $D_{e q}$ is previously known and it should bring advantages in dose evaluation for clinical CT examinations.
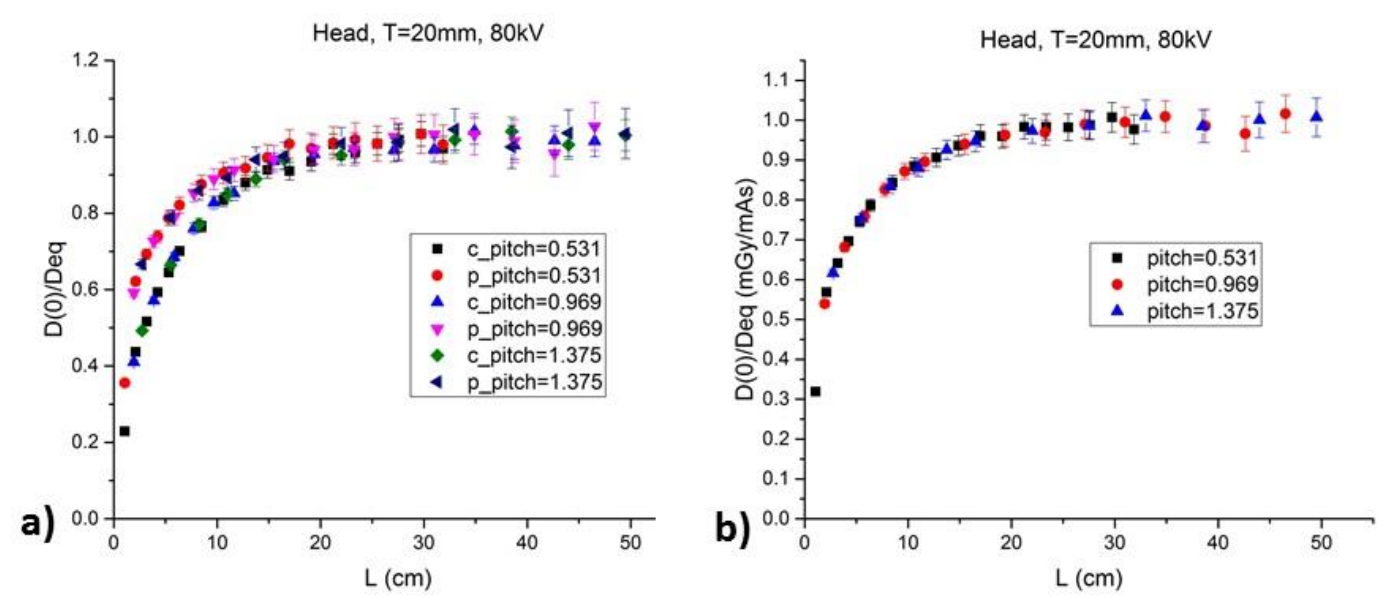

Figure 78. Ratio Maximum Dose at $\mathrm{z}=0$ to Equilibrium $\operatorname{Dose}, D_{L}(0) / D_{e q}$ at a) central and peripheral positions and b) weighted by the $C T D I_{w}$ coefficients for pitch values $0.531,0.969$ and 1.375 at $80 \mathrm{kV}$.
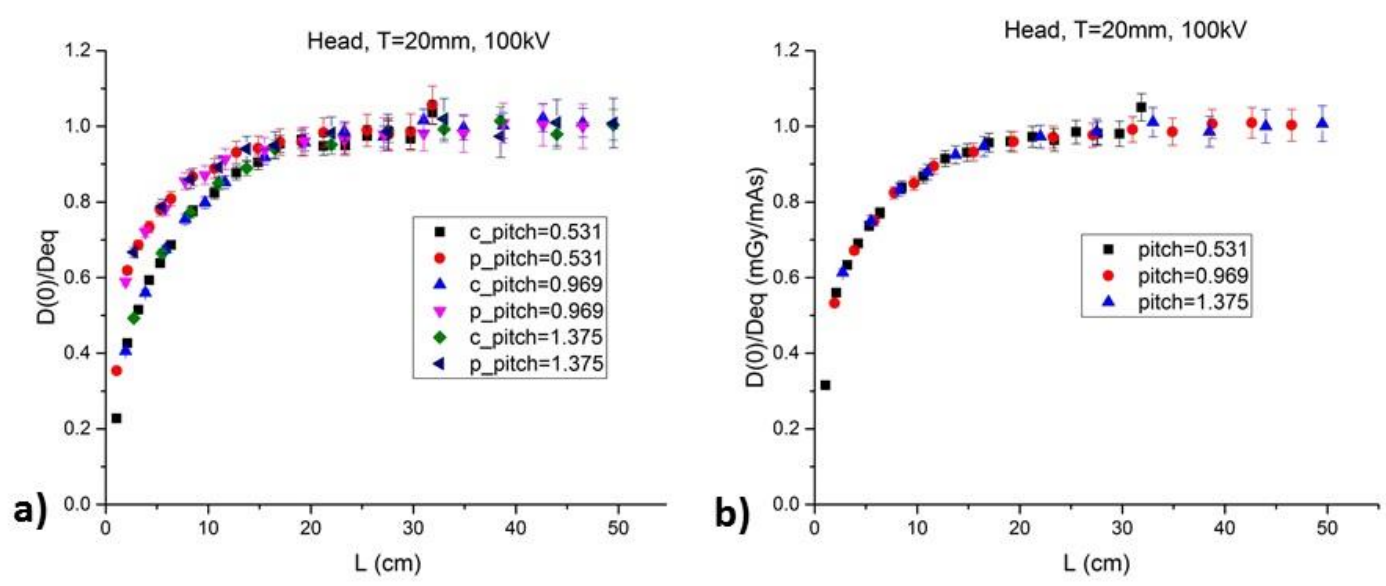

Figure 79. Ratio Maximum Dose at $\mathrm{z}=0$ to Equilibrium $\operatorname{Dose}, D_{L}(0) / D_{e q}$ at a) central and peripheral positions and b) weighted by the $C T D I_{w}$ coefficients for pitch values $0.531,0.969$ and 1.375 at $100 \mathrm{kV}$. 

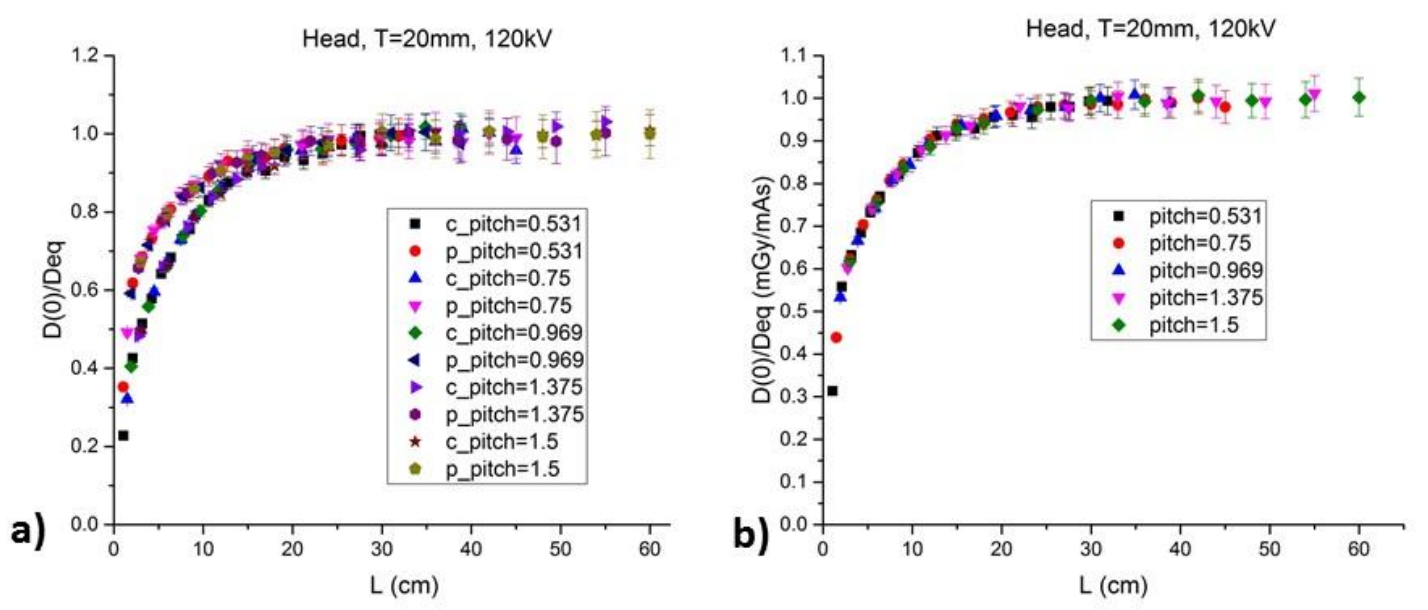

Figure 80. Ratio Maximum Dose at $\mathrm{z}=0$ to Equilibrium Dose, $D_{L}(0) / D_{e q}$ at a) central and peripheral positions and b) weighted by the $C T D I_{w}$ coefficients for pitch values $0.531,0.969$ and 1.375 at $120 \mathrm{kV}$.
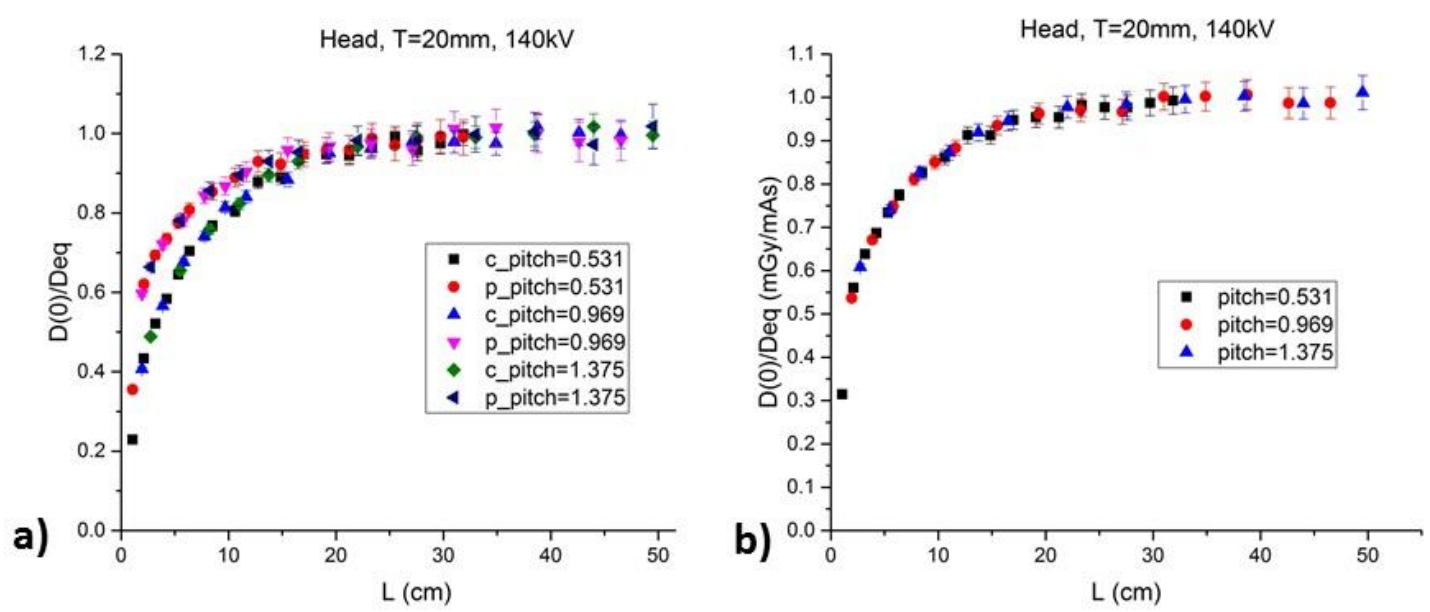

Figure 81. Ratio Maximum Dose at $\mathrm{z}=0$ to Equilibrium Dose, $D_{L}(0) / D_{e q}$ at a) central and peripheral positions and b) weighted by the $C T D I_{w}$ coefficients for pitch values $0.531,0.969$ and 1.375 at $140 \mathrm{kV}$.
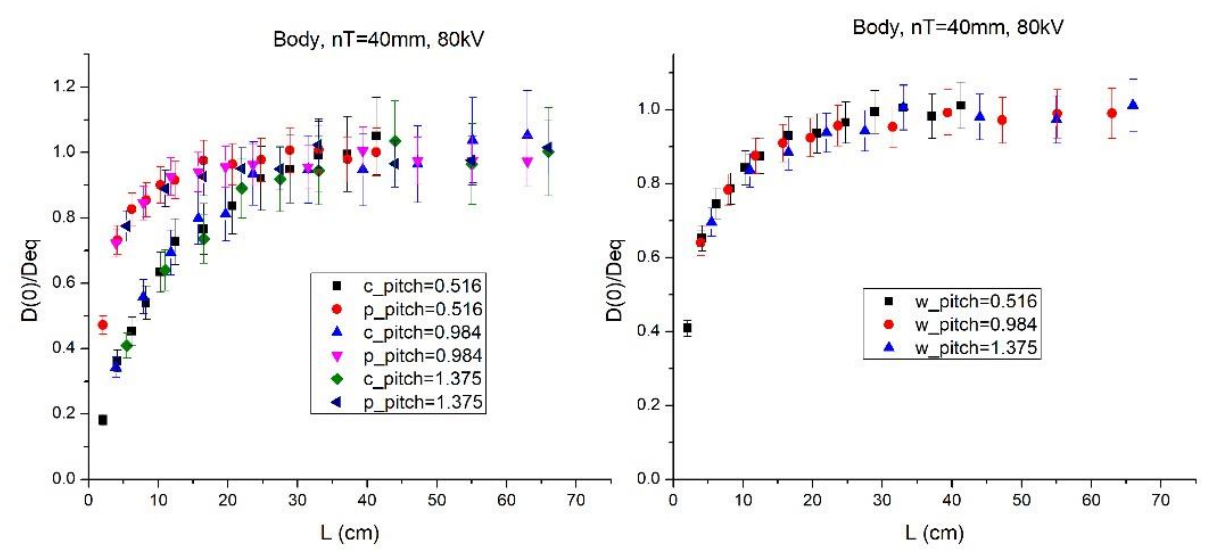

Figure 82. Ratio Maximum Dose at $\mathrm{z}=0$ to Equilibrium Dose, $D_{L}(0) / D_{e q}$ at a) central and peripheral positions and b) weighted by the $C T D I_{w}$ coefficients for pitch values $0.531,0.969$ and 1.375 at $140 \mathrm{kV}$. 

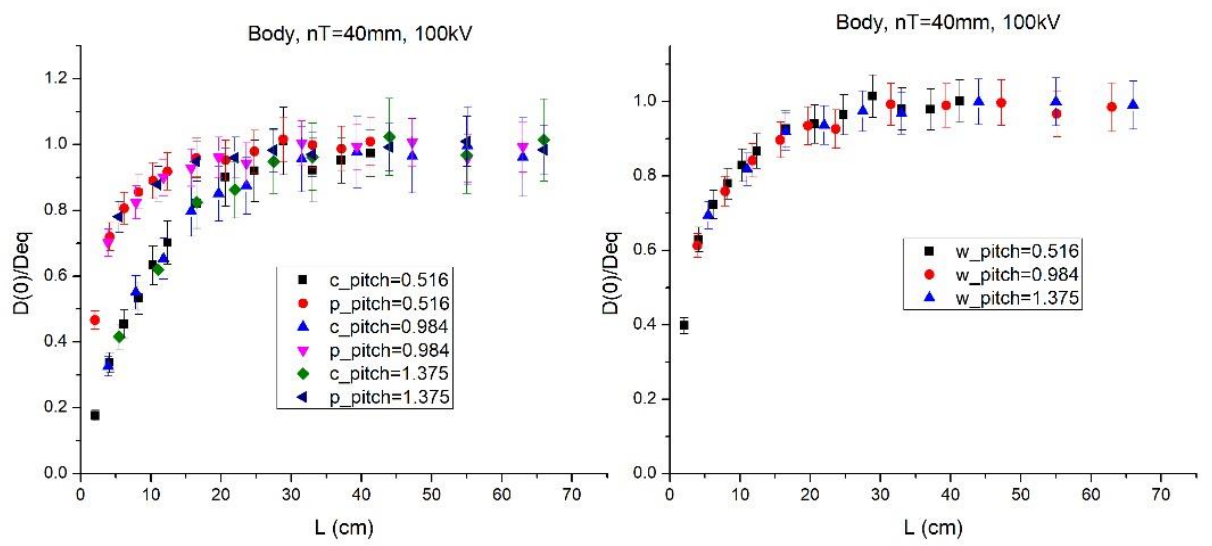

Figure 83. Ratio Maximum Dose at $\mathrm{z}=0$ to Equilibrium Dose, $D_{L}(0) / D_{e q}$ at a) central and peripheral positions and b) weighted by the $C T D I_{w}$ coefficients for pitch values $0.531,0.969$ and 1.375 at $140 \mathrm{kV}$.
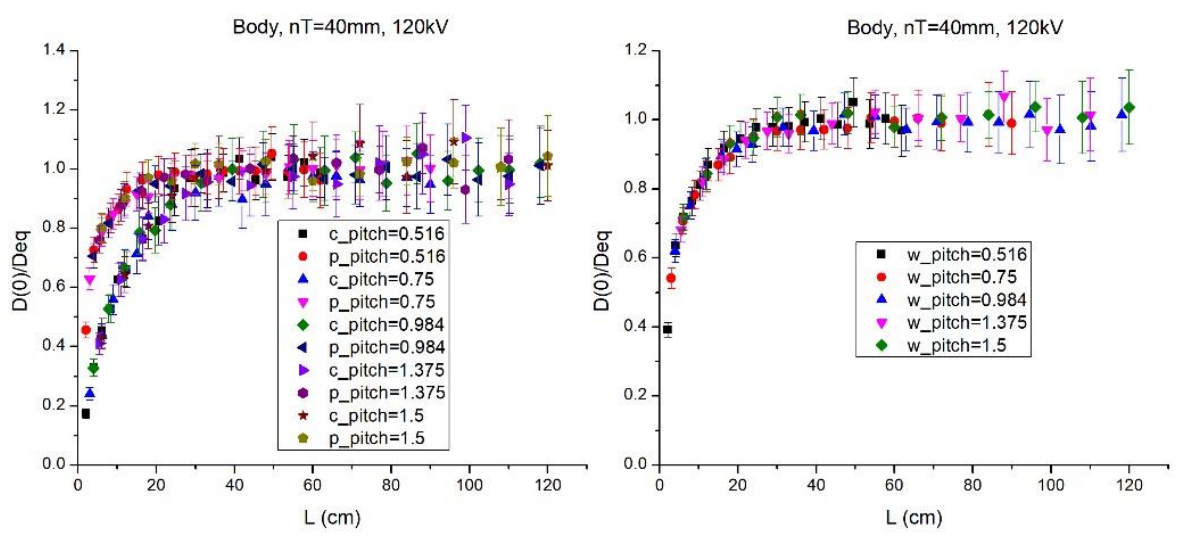

Figure 84. Ratio Maximum Dose at $\mathrm{z}=0$ to Equilibrium Dose, $D_{L}(0) / D_{e q}$ at a) central and peripheral positions and b) weighted by the $C T D I_{w}$ coefficients for pitch values $0.531,0.969$ and 1.375 at $140 \mathrm{kV}$.
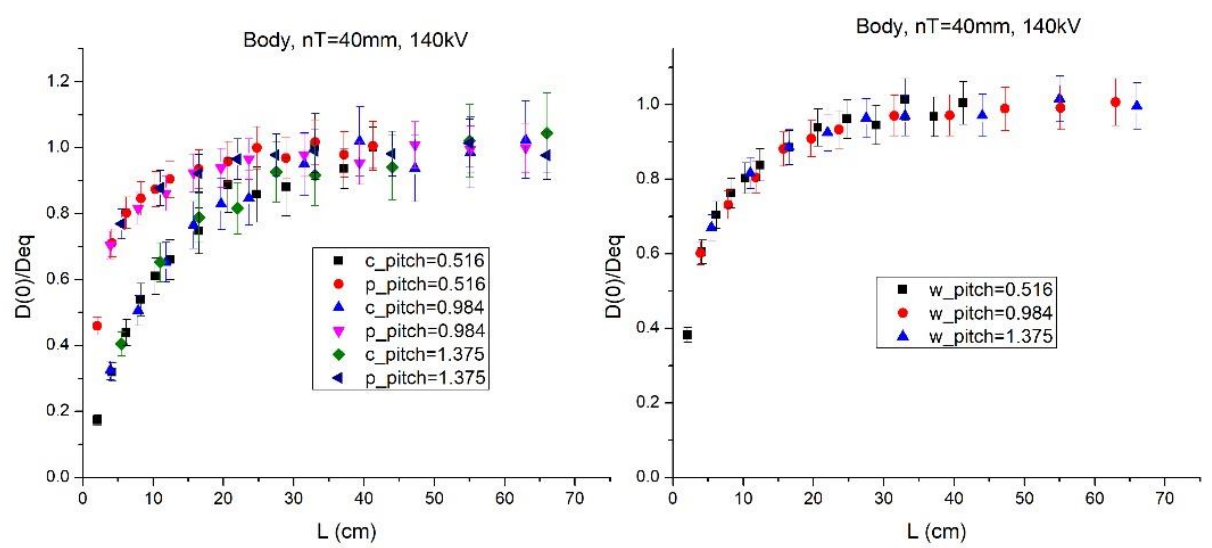

Figure 85. Ratio Maximum Dose at $\mathrm{z}=0$ to Equilibrium Dose, $D_{L}(0) / D_{e q}$ at a) central and peripheral positions and b) weighted by the $C T D I_{w}$ coefficients for pitch values $0.531,0.969$ and 1.375 at $140 \mathrm{kV}$. 
$H(L)$ curves suggest fitting with a parametric model related for the analytical expression for $H(L)$ presented in Equation (52). In this work the parametric model proposed in AAPM report 111 (AAPM, 2010) shown in Equation (64) is used to fit the approach-toequilibrium function.

$$
H(L)=1-\alpha \cdot \exp \left(-\frac{4 L}{L_{e q}}\right)
$$

Where, $\alpha$ and $L_{e q}$ are parameters assessed by the Origin's NLFit tool (Nonlinear Curve Fitting) of the OriginPro 8.6 software (OriginLab Corporation, Northampton, MA, USA). The parameter $\alpha$ is the scatter fraction at the $D_{e q}$, which is related to the scatter to primary ratio, SPR (Boone, 2009), at the $D_{e q}$ by $\alpha=S P R /(1+S P R)$. By convention, $L_{e q}$ is the scan length where $H(L)=0.98$. In an infinitely long cylinder, both $D_{e q}$ and $L_{e q}$ depend on radial distance to the central axis. The fitting was performed for each set of points corresponding to a specific pitch value, tube voltage, CTDI phantom, and for a center, peripheral, or weighted $H(L)$. The graphs with the corresponding fitting line and performance and statistics of the fitting are shown in Appendix E. Table 19 and Table 20 present the estimative of both parameters, $\alpha$ and $L_{e q}$, for the head and body helical protocols, respectively. In Table 19, the center, peripheral, and weighted $L_{e q}$ has small dependence with the X-ray spectra and pitch values for the head helical protocol (or head bowtie filter). Analog conclusion could be performed for the parameter $\alpha$ for the head helical protocol. For the body helical protocol, the parameters $L_{e q}$ and $\alpha$ have small dependence with the X-ray spectra and pitch values only for the central axis, variations for the peripheral axis and as a consequence for the weighted case are identified.

The introduction of the $L_{e q}$, helps to evaluate dose precluding measurements in long cylindrical phantoms or the use of long pencil ion chambers. Dependence with the pitch value for both $\alpha$ and $L_{e q}$ appear to be no negligible for the peripheral profile in the body phantom. Major variation of $L_{e q}$ for head helical protocols are, $1.8 \mathrm{~cm}$ (center at $140 \mathrm{kV}$ ), $3.8 \mathrm{~cm}$ (peripheral at $100 \mathrm{kV}$ ), and $2.1 \mathrm{~cm}$ (weighted at $100 \mathrm{kV}$ ), data derived from Table 19. Major variation of $L_{e q}$ for body helical protocols are: $5.4 \mathrm{~cm}$ (center at $120 \mathrm{kV}$ ), $13.3 \mathrm{~cm}$ (peripheral at $80 \mathrm{kV}$ ), and $9.4 \mathrm{~cm}$ (weighted at $80 \mathrm{kV}$ ), data derived from Table 20. It is interesting to note that the parameter $\alpha$ is higher for the central axis than for the peripheral axis for both head and body phantom. It makes sense since the contribution of the scatter radiation to the 
deposited dose at the central region is higher than at the periphery. The scatter fraction $\alpha$ at the central axis for the body phantom case is higher than for the head phantom case. Again, it makes sense since the X-ray beam is more scattered by the major phantom material in the body than in the head case. It is interesting to note that the parameter $\alpha$ is insensitive with the tube voltage, for the head and body protocols, as shown by Xinhua Li (Li et al., 2013). But, $\alpha$ has a small dependence with the pitch value that was not evaluated by Xinhua Li.

Table 19. Equilibrium scan length, $L_{e q}$, and scatter fraction, $\alpha$, for head helical protocol.

\begin{tabular}{llllllll}
\hline & \multicolumn{3}{c}{ center } & \multicolumn{2}{c}{ Peripheral } & \multicolumn{2}{c}{ Weighted } \\
\hline Voltage & Pitch & $\boldsymbol{L}_{\boldsymbol{e q}}(\mathrm{cm})$ & $\boldsymbol{\alpha}$ & $\boldsymbol{L}_{\boldsymbol{e q}}(\mathrm{cm})$ & $\boldsymbol{\alpha}$ & $\boldsymbol{L}_{\boldsymbol{e q}}(\mathrm{cm})$ & $\boldsymbol{\alpha}$ \\
\hline \multirow{3}{*}{$\mathbf{8 0 k V}$} & $\mathbf{0 . 5 3 1}$ & $28.1(0.7)$ & $0.76(1)$ & $24.1(0.8)$ & $0.53(1)$ & $25.6(0.6)$ & $0.59(1)$ \\
& $\mathbf{0 . 9 6 9}$ & $27.2(1.2)$ & $0.75(3)$ & $27.4(2.3)$ & $0.48(4)$ & $27.3(1.4)$ & $0.56(3)$ \\
& $\mathbf{1 . 3 7 5}$ & $27.5(0.7)$ & $0.76(1)$ & $26.8(1.4)$ & $0.50(2)$ & $27.1(0.8)$ & $0.57(1)$ \\
\hline \multirow{3}{*}{$\mathbf{1 0 0 k V}$} & $\mathbf{0 . 5 3 1}$ & $27.7(0.8)$ & $0.77(1)$ & $25.7(1.3)$ & $0.52(2)$ & $26.5(1.0)$ & $0.60(1)$ \\
& $\mathbf{0 . 9 6 9}$ & $27.6(1.0)$ & $0.77(2)$ & $29.5(1.6)$ & $0.47(2)$ & $28.6(1.0)$ & $0.56(2)$ \\
& $\mathbf{1 . 3 7 5}$ & $27.5(0.7)$ & $0.76(1)$ & $26.8(1.4)$ & $0.50(2)$ & $27.1(0.7)$ & $0.58(1)$ \\
\hline & $\mathbf{0 . 5 3 1}$ & $29.7(0.7)$ & $0.76(1)$ & $27.7(1.0)$ & $0.50(1)$ & $28.3(0.8)$ & $0.58(1)$ \\
$\mathbf{1 2 0 k V}$ & $\mathbf{0 . 7 5}$ & $28.1(0.8)$ & $0.77(2)$ & $26.9(1.0)$ & $0.49(1)$ & $27.4(0.7)$ & $0.58(1)$ \\
& $\mathbf{0 . 9 6 9}$ & $28.1(1.0)$ & $0.77(2)$ & $30.1(1.4)$ & $0.47(2)$ & $29.1(0.8)$ & $0.57(1)$ \\
& $\mathbf{1 . 3 7 5}$ & $28.5(1.0)$ & $0.76(2)$ & $27.6(1.1)$ & $0.51(1)$ & $27.9(0.8)$ & $0.59(1)$ \\
& $\mathbf{1 . 5}$ & $29.5(0.8)$ & $0.75(1)$ & $28.9(1.0)$ & $0.49(1)$ & $29.1(0.6)$ & $0.57(1)$ \\
\hline \multirow{2}{*}{$\mathbf{1 4 0 k V}$} & $\mathbf{0 . 5 3 1}$ & $28.7(0.8)$ & $0.75(1)$ & $28.0(1.0)$ & $0.50(1)$ & $28.3(0.8)$ & $0.58(1)$ \\
& $\mathbf{0 . 9 6 9}$ & $30.5(1.2)$ & $0.71(2)$ & $28.8(2.0)$ & $0.48(3)$ & $29.5(1.0)$ & $0.55(2)$ \\
& $\mathbf{1 . 3 7 5}$ & $28.9(0.6)$ & $0.75(1)$ & $27.3(1.3)$ & $0.50(2)$ & $27.9(0.6)$ & $0.58(1)$ \\
\hline
\end{tabular}

Table 20. Equilibrium scan length, $L_{e q}$, and scatter fraction, $\alpha$, for body helical protocol.

\begin{tabular}{|c|c|c|c|c|c|c|c|}
\hline \multirow[b]{2}{*}{ Voltage } & \multirow[b]{2}{*}{ Pitch } & \multicolumn{2}{|c|}{ center } & \multicolumn{2}{|c|}{ Peripheral } & \multicolumn{2}{|c|}{ Weighted } \\
\hline & & $\boldsymbol{L}_{\boldsymbol{e q}}(\mathrm{cm})$ & $\alpha$ & $\boldsymbol{L}_{\boldsymbol{e q}}(\mathrm{cm})$ & $\alpha$ & $\boldsymbol{L}_{e q}(\mathrm{~cm})$ & $\alpha$ \\
\hline \multirow{3}{*}{$80 \mathrm{kV}$} & 0.516 & $44.0(2.3)$ & $0.94(3)$ & $26.1(1.9)$ & $0.49(3)$ & $32.6(1.5)$ & $0.57(2)$ \\
\hline & 0.984 & $41.7(2.1)$ & $0.96(3)$ & $29.5(3.4)$ & $0.47(4)$ & $34.5(2.4)$ & $0.56(3)$ \\
\hline & 1.375 & $46.1(2,9)$ & $0.95(4)$ & $39.4(6.4)$ & $0.39(5)$ & $42.0(3.7)$ & $0.51(4)$ \\
\hline \multirow{3}{*}{$100 \mathrm{kV}$} & 0.516 & $41.0(1.1)$ & $1.00(3)$ & $26.7(1.7)$ & $0.51(3)$ & $32.1(1.4)$ & $0.61(2)$ \\
\hline & 0.984 & $42.7(1.5)$ & $0.97(2)$ & $32.0(2.8)$ & $0.48(3)$ & $36.3(2.0)$ & $0.59(2)$ \\
\hline & 1.375 & $42.8(2.8)$ & $0.98(4)$ & $36.0(3.0)$ & $0.40(3)$ & $38.7(2.1)$ & $0.54(3)$ \\
\hline \multirow{5}{*}{$120 \mathrm{kV}$} & 0.516 & $43.9(2.2)$ & $0.98(3)$ & $28.9(2.3)$ & $0.49(3)$ & $34.7(1.7)$ & $0.59(2)$ \\
\hline & 0.75 & $44.4(1.9)$ & $0.99(2)$ & $31.1(2.2)$ & $0.53(3)$ & $36.1(1.8)$ & $0.62(2)$ \\
\hline & 0.984 & $45.0(2.1)$ & $0.95(2)$ & $35.0(2.9)$ & $0.46(3)$ & $39.0(1.9)$ & $0.57(2)$ \\
\hline & 1.375 & $48.9(3.8)$ & $0.92(5)$ & $35.8(6.1)$ & $0.44(7)$ & $41.0(3.6)$ & $0.54(4)$ \\
\hline & 1.5 & $43.5(3.7)$ & $0.99(6)$ & $30.2(5.7)$ & $0.44(9)$ & $35.4(3.7)$ & $0.56(5)$ \\
\hline \multirow{3}{*}{$140 \mathrm{kV}$} & 0.516 & $45.8(2.3)$ & $0.97(3)$ & $29.8(2.2)$ & $0.49(3)$ & $37.0(2.1)$ & $0.60(3)$ \\
\hline & 0.984 & $47.2(1.9)$ & $0.95(2)$ & $38.7(2.8)$ & $0.44(2)$ & $42.3(1.1)$ & $0.58(1)$ \\
\hline & 1.375 & $46.3(3.3)$ & $0.95(4)$ & $37.2(2.8)$ & $0.42(3)$ & $41.7(2.2)$ & $0.55(2)$ \\
\hline
\end{tabular}


Table 21 and Table 22 show the SPR computed from the scatter fraction $\alpha$ using the relation $\alpha=S P R /(1+S P R)$ for head and body protocols, respectively. In Table 21, the SPR computed from pitches, $0.516,0.969$ and 1.375 appear to be insensitive for the pitches and Xray spectra for each central, peripheral, and weight cases. In Table 21, the SPR at central axis is approximately three times the one at the peripheral axis. It means that at the central axis the radiation scattered by the phantom material has more contribution to the dose than the produced by the primary beam. Values for SPR at the central axis are very high with big uncertainties. The reason may be referred to the fitting quality, as can be seen from the $\alpha$ values in Table 20 in which its values approximately to 1 do not make sense. The scatter fraction equal to 1 refers that only the scatter radiation contributes to the dose. In the case of the peripheral axis, the to the dose form the scatter radiation and the primary beam appear to be similar, as the values of SPR close to 1 suggests, for the cases with low uncertainties.

Table 21. Scatter to primary ratio, SPR, for head helical protocol.

\begin{tabular}{lccccccc}
\hline & \multicolumn{3}{c}{ center } & \multicolumn{2}{c}{ Peripheral } & \multicolumn{2}{c}{ Weight } \\
\hline Voltage & Pitch & $\boldsymbol{S P R}(\boldsymbol{\sigma})$ & $\%$ & $\boldsymbol{S P R}(\boldsymbol{\sigma})$ & $\%$ & $\boldsymbol{S P R}(\boldsymbol{\sigma})$ & $\%$ \\
\hline \multirow{2}{*}{$\mathbf{8 0 k V}$} & $\mathbf{0 . 5 3 1}$ & $3,2(2)$ & 5.5 & $1.13(5)$ & 4.0 & $1.4(1)$ & 4.1 \\
& $\mathbf{0 . 9 6 9}$ & $3.0(5)$ & 16.0 & $0.9(1)$ & 16.0 & $1.3(2)$ & 12.2 \\
& $\mathbf{1 . 3 7 5}$ & $3.2(2)$ & 5.5 & $1.00(8)$ & 8.0 & $1.33(5)$ & 4.1 \\
\hline \multirow{3}{*}{$\mathbf{1 0 0 k V}$} & $\mathbf{0 . 5 3 1}$ & $3.3(2)$ & 5.6 & $1.1(1)$ & 8.0 & $1.5(1)$ & 4.2 \\
& $\mathbf{0 . 9 6 9}$ & $3.3(4)$ & 11.3 & $0.9(1)$ & 8.0 & $1.27(1)$ & 8.1 \\
& $\mathbf{1 . 3 7 5}$ & $3.2(2)$ & 5.5 & $1.00(8)$ & 8.0 & $1.38(6)$ & 4.1 \\
\hline & $\mathbf{0 . 5 3 1}$ & $3.2(2)$ & 5.5 & $1.00(4)$ & 4.0 & $1.4(1)$ & 4.1 \\
$\mathbf{1 2 0 k V}$ & $\mathbf{0 . 7 5}$ & $3.3(4)$ & 11.3 & $0.96(4)$ & 4.0 & $1.4(1)$ & 4.1 \\
& $\mathbf{0 . 9 6 9}$ & $3.3(4)$ & 11.3 & $0.89(7)$ & 8.0 & $1.33(5)$ & 4.1 \\
& $\mathbf{1 . 3 7 5}$ & $3.2(3)$ & 11.0 & $1.04(4)$ & 4.0 & $1.44(6)$ & 4.1 \\
& $\mathbf{1 . 5}$ & $3.0(2)$ & 5.3 & $0.96(4)$ & 4.0 & $1.33(5)$ & 4.1 \\
\hline \multirow{2}{*}{$\mathbf{1 4 0 k V}$} & $\mathbf{0 . 5 3 1}$ & $3.0(2)$ & 5.3 & $1.00(4)$ & 4.0 & $1.4(1)$ & 4.1 \\
& $\mathbf{0 . 9 6 9}$ & $2.4(2)$ & 9.7 & $0.9(1)$ & 12.0 & $1.2(1)$ & 8.1 \\
& $\mathbf{1 . 3 7 5}$ & $3.0(2)$ & 5.3 & $1.00(8)$ & 8.0 & $1.38(6)$ & 4.1 \\
\hline
\end{tabular}

Table 22. Scatter to primary ratio, SPR, for body helical protocol.

\begin{tabular}{cccccc}
\hline & \multicolumn{3}{c}{ Peripheral } & \multicolumn{2}{c}{ Weighted } \\
\hline Voltage & Pitch & $\boldsymbol{S P R}(\boldsymbol{\sigma})$ & $\%$ & $\boldsymbol{S P R}(\boldsymbol{\sigma})$ & $\%$ \\
\hline \multirow{2}{*}{$\mathbf{0 0 k V}$} & $\mathbf{0 . 5 1 6}$ & $1.0(1)$ & 12.0 & $1.3(1)$ & 8.2 \\
& $\mathbf{0 . 9 8 4}$ & $0.9(1)$ & 16.1 & $1.3(2)$ & 12.2 \\
& $\mathbf{1 . 3 7 5}$ & $0.6(1)$ & 21.0 & $1.0(2)$ & 16.0 \\
\hline \multirow{2}{*}{$\mathbf{1 0 0 k V}$} & $\mathbf{0 . 5 1 6}$ & $1.0(1)$ & 12.0 & $1.6(1)$ & 8.4 \\
& $\mathbf{0 . 9 8 4}$ & $0.9(1)$ & 12.0 & $1.4(1)$ & 8.3 \\
& $\mathbf{1 . 3 7 5}$ & $0.67(8)$ & 12.5 & $1.2(1)$ & 12.1 \\
\hline & $\mathbf{0 . 5 1 6}$ & $1.0(1)$ & 12.0 & $1.4(1)$ & 8.3 \\
$\mathbf{1 2 0 k V}$ & $\mathbf{0 . 7 5}$ & $1.1(1)$ & 12.0 & $1.6(1)$ & 8.5 \\
& $\mathbf{0 . 9 8 4}$ & $0.9(1)$ & 12.1 & $1.3(1)$ & 8.2 \\
& $\mathbf{1 . 3 7 5}$ & $0.8(2)$ & 28.4 & $1.2(2)$ & 16.1 \\
& $\mathbf{1 . 5}$ & $0.8(3)$ & 36.5 & $1.3(3)$ & 20.3 \\
\hline & $\mathbf{0 . 5 1 6}$ & $1.0(1)$ & 12.0 & $1.5(2)$ & 12.5 \\
\hline
\end{tabular}




\begin{tabular}{lllccc}
$140 k V$ & $\mathbf{0 . 9 8 4}$ & $0.79(6)$ & 8.1 & $1.38(6)$ & 4.1 \\
& $\mathbf{1 . 3 7 5}$ & $0.72(9)$ & 12.3 & $1.2(1)$ & 8.1 \\
\hline
\end{tabular}

Table 23 and Table 24 compare the SPR and $L_{e q}$ results from this work with data from the literature, respectively. In the case of SPR for the head CTDI phantom, good agreement is reached with data from (Li et al., 2013) and (Tsai et al., 2003) for the central and peripheral axes. The data from the literature were computed or measured for pitch value equal 1. For the body phantom the SPR shows some coherence for the peripheral axis only. Comparison of the $L_{e q}$ in the case of body CTDI phantom (32 cm diameter) at the central axis shows good agreement with data from MC simulation (Li et al., 2013, Dixon and Boone, 2010) and experimental measurements (Dixon and Ballard, 2007), as shown in Table 24. In the case of peripheral axis, $L_{e q}$ from this work differs in approximately $5 \mathrm{~cm}$ with respect to data from literature. It is important to mention that data from ( $\mathrm{Li}$ et al., 2013) was derived using a $1 \mathrm{~mm}$ beam width. Nevertheless, $\mathrm{Li}$ et al. show that the variation of $L_{e q}$ with the beam width (from 1 to $40 \mathrm{~mm}$ ) is less than $1 \mathrm{~cm}$ at $120 \mathrm{kV}$, which is covered by the uncertainty from their results. Data from (Li et al., 2013) was derived for direct integration of the single dose profile and they considered pitch=1. Data from Dixon and Ballard (Dixon and Ballard, 2007) was acquired at $120 \mathrm{kV}, 400 \mathrm{mAx} 1 \mathrm{~s}$, large FOV, large focal spot, $n T=64 \times 0.625 \mathrm{~mm}=40 \mathrm{~mm}$ ( $p=0.984$ on AOR and $p=0.516$ on periphery), $n T=32 \times 0.625 \mathrm{~mm}=20 \mathrm{~mm}(p=0.969$ on AOR and $p=0.531$ on periphery). Also in Table 24 , for the head CTDI phantom case at the central axis, $L_{e q}$ form this work and the one computed by Li et al. (Li et al., 2013) are in good agreement.

Table 23. Scatter to primary ratio, SPR, for head and body helical protocols.

\begin{tabular}{ccccccc}
\hline Entry & Diameter & Location & This work & $\begin{array}{c}\text { (Li et al., } \\
\mathbf{2 0 1 3})\end{array}$ & $\begin{array}{c}\text { (Tsai et } \\
\text { al., 2003) }\end{array}$ & $\begin{array}{c}\text { (Dixon and } \\
\text { Boone, 2010) }\end{array}$ \\
\hline \multirow{3}{*}{ SPR } & $\mathbf{1 6}$ & Periphery & $0.9-1.13$ & 1.21 & $0.9-1.3$ & $\ldots$ \\
& $\mathbf{1 6}$ & Center & $3.0-3.3$ & 3.78 & 3.0 & $\ldots$ \\
\cline { 2 - 7 } & $\mathbf{3 2}$ & Periphery & $0.6-1.1$ & 1.23 & $0.8-1.1$ & 1.5 \\
& $\mathbf{3 2}$ & Center & $19(8)$ & 8.21 & 5.7 & 13.0 \\
\hline
\end{tabular}

Table 24. Equilibrium scanning length, $L_{e q}$, for head and body helical protocols.

\begin{tabular}{ccccccc}
\hline Entry & $\begin{array}{c}\text { Diameter } \\
(\mathbf{c m})\end{array}$ & Location & This work & $\begin{array}{c}\text { (Li et al., } \\
\mathbf{2 0 1 3})\end{array}$ & $\begin{array}{c}\text { (Dixon and } \\
\text { Ballard, 2007) }\end{array}$ & $\begin{array}{c}\text { (Dixon and } \\
\text { Boone, 2010) }\end{array}$ \\
\hline \multirow{3}{*}{$\boldsymbol{L}_{\boldsymbol{e q}}$} & $\mathbf{1 6}$ & Periphery & $30.1(1.4)$ & $24.1(1.0)$ & --- & --- \\
$(\mathbf{c m})$ & Center & $28.1(1.0)$ & $28.5(1.0)$ & --- & -- \\
& $\mathbf{3 2}$ & Periphery & $35.0(2.9)$ & $28.4(1.0)$ & 28.0 & 30.0 \\
& $\mathbf{3 2}$ & Center & $45.0(2.1)$ & $42.0(1.0)$ & 44.8 & 47.0 \\
\hline
\end{tabular}


It is important to note that data from Table 19 and Table 20 were computed by fitting curves from cumulated dose profiles from difference pitch values. Therefore, they incorporate the CT table translation. While $D_{e q}$ substantially increased with the tube voltage, $L_{e q}$ only slightly increased with the tube voltage, for the $\mathrm{kV}$ settings evaluated in this work.

\subsection{Evaluation of the efficiency of the $C T D I_{100}$ method}

\subsubsection{The product pitch $x$ Equilibrium dose versus $C T D I_{100}$}

The product pitch $\mathrm{x}$ Equilibrium dose is equal to the original definition of the CTDI, as presented in Equation (65).

$$
\widehat{D}_{e q}=p \cdot D_{e q}=\frac{1}{n T} \int_{-\infty}^{\infty} f(z) d z=C T D I
$$

Therefore, a direct comparison between $\widehat{D}_{e q}$ and the $C T D I_{100}$ represent an evaluation of the efficiency of the $C T D I_{100}$ to estimate CTDI or indirectly the $D_{e q}$ for any value of pitch and helical protocols, since the product $p . D_{e q}$ is constant for any value of pitch as shown in Table 16 and Table 17. Table 25 presents the efficiency in terms of percentage of the CTDI 100 to assess the product p. $D_{e q}$ (or CTDI). In the case of $120 \mathrm{kV}$, the efficiency is $80.6 \%$ and $87.8 \%$ for the central and peripheral axes of the head phantom, respectively. In case of body phantom, the efficiency at the central axis is reduced to $56.5 \%$ and at the peripheral axis is $86.2 \%$ for $120 \mathrm{kV}$.

Table 25. The product Pitch $x$ Equilibrium Dose, $p x D_{e q}$ versus $C T D I_{100}$ for head $(n T=20 \mathrm{~mm})$ and body $(n T=40 \mathrm{~mm})$ helical protocols.

\begin{tabular}{|c|c|c|c|c|c|c|}
\hline \multirow[b]{3}{*}{ Voltage } & \multicolumn{6}{|c|}{$\%$} \\
\hline & \multicolumn{3}{|c|}{ Head } & \multicolumn{3}{|c|}{ Body } \\
\hline & $\begin{array}{l}C T D I_{100, c} \\
/ p x D_{e q, c}\end{array}$ & $\begin{array}{l}C T D I_{100, p} \\
/ p x D_{e q, p}\end{array}$ & $\begin{array}{l}C T D I_{w} \\
/ p x D_{e q, w}\end{array}$ & $\begin{array}{l}C T D I_{100, c} \\
/ p x D_{e q, c}\end{array}$ & $\begin{array}{l}C T D I_{100, p} \\
/ p x D_{e q, p}\end{array}$ & $\begin{array}{l}C T D I_{w} \\
/ p x D_{e q, w}\end{array}$ \\
\hline $80 \mathrm{kV}$ & 82.1 & 89.5 & 87.3 & 59.5 & 89.1 & 82.6 \\
\hline 100kV & 80.8 & 88.7 & 86.2 & 57.4 & 87.1 & 80.0 \\
\hline $120 \mathrm{kV}$ & 80.6 & 87.8 & 85.5 & 56.5 & 86.2 & 79.4 \\
\hline $140 \mathrm{kV}$ & 80.5 & 88.1 & 85.7 & 56.2 & 86.1 & 78.0 \\
\hline
\end{tabular}

\subsubsection{Dose line integral versus dose length product}

DLI represent an improvement to the DLP to estimate dose delivered by a CT scanner. DLI comes from total integration of the cumulative dose profile, as indicated in Equation (66). Figure 86 show a comparison between the Dose Line Integral versus Dose 
Length Product, as can be seen, the limitation of the $C T D I_{100}$ to estimate the CT radiation delivered is reflect in its derivative quantity DLP.

$$
D L I=\int_{-\infty}^{\infty} D_{L}(z) d z
$$

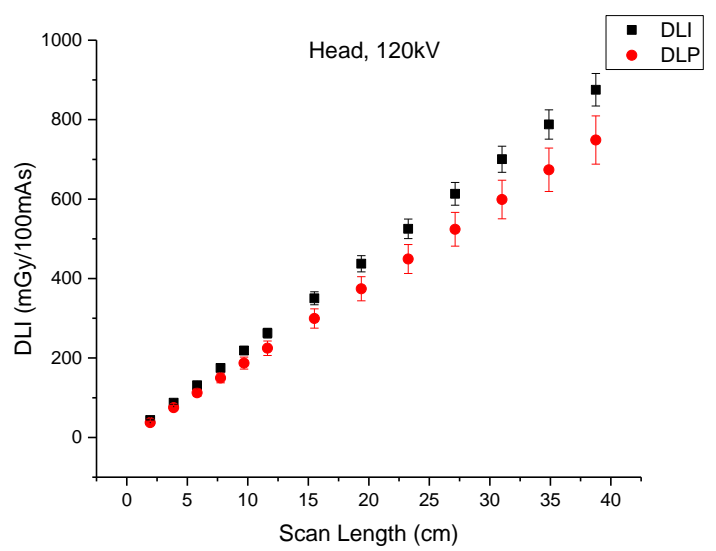

Figure 86. Dose Line Integral versus Dose Length Product for head CTDI phantom at 120 kV. 


\section{Chapter 5 - Conclusions}

In this work, cumulative dose profiles in computed tomography (CT) were obtained from Monte Carlo (MC) simulation of coupled electron-photon transport in cylindrical phantoms of polymethyl methacrylate (PMMA). The radiation emission characteristics of a multiple-row detector CT (MDCT) were modelled using the PENELOPE/penEasy MC software. Clinical helical protocols used by the CT scanners GE Discovery CT750 HD and GE Lightspeed Ultra were considered to compute cumulative profiles along the central and peripheral axes of the PMMA cylindrical phantoms of $16 \mathrm{~cm}$ (head) and $32 \mathrm{~cm}$ (body) diameters. CT dose metrics proposed in the AAPM report 111 (AAPM, 2010) and supported by the analytical formulation developed by Dixon et al. (Dixon, 2019) were derived from the simulated cumulative profiles.

The modelled CT X-ray source incorporated the anode self-attenuation, focal spot angle, and X-ray spectrum. The last mentioned issues affect the shape of the primary dose profile produced by a single axial rotation. The primary profiles from Monte Carlo simulation showed good agreement with the analytical ones, as shown in Figure 52. Also, the modelled CT X-ray source incorporates the modulation of the X-ray beam on the axial plane due to the bowtie filter attenuation. The performance of the bowtie filter modelling was evaluated by comparing the ratios $C T D I_{100, c} / C T D I_{100, p}$ obtained from MC simulation with experimental measurements and data from CTDosimetry datasheet. Percentage difference with respect to the experimental data up to $23.4 \%(n T=10 \mathrm{~mm})$ and $52.9 \%(n T=20 \mathrm{~mm})$ for $80 \mathrm{kV}$ X-ray spectrum and head bowtie filter, and up to $8.9 \%(n T=10 \mathrm{~mm})$ and $11.6 \%(n T=40 \mathrm{~mm})$ for $140 \mathrm{kV}$ and $120 \mathrm{kV} \mathrm{X}$-ray spectra, respectively, for body bowtie filter were found. With respect to the X-ray spectra, they were generated by the semi-empirical TBC model (Costa et al., 2007, Lopez Gonzales et al., 2015) following the spectra characteristics measured by indirect methods (Terini et al., 2017). The division of the whole MC simulation process of the CT helical scan modes into two stages showed benefits in terms of simulation time and disk storage administrations, also, allowed good performance in error identifications in the MC modelling process.

A number of cumulative dose profiles computed by MC simulation of the CT helical scan mode were presented. The cumulative profile shape depended on the CT acquisition parameters, it was especially influenced by the scan length $(L)$, the beam width $(a)$, and pitch value $(p)$. In general, the central cumulative profile shape is mainly conformed by the scatter radiation which its contribution is around 3.3 times the contribution of the primary radiation 
for the head phantom, as can be deduced from the scatter to primary ratio (SPR) values. The peripheral cumulative profile shape has roughly similar contributions of the primary and scatter radiation. Its SPR was found as approximately 1 for both head and body phantom. Therefore, the shape of the peripheral profile appears to be influenced by the pitch. For $p>1$, the profile shape presents valleys because there are regions along the peripheral axis that are not irradiated directly by the X-ray beam. For $p<1$, there are superposition of the primary beam along the scan length on the peripheral axis that produce peaks in the cumulative profiles. Some differences between the cumulative profiles computed by MC simulation with respect to the ones measured with OSL ribbons were identified. For $p>1$, the measured cumulative profiles shape present more pronounced valleys with respect to the simulated ones. It could be produced by the influence of the CT table attenuation that in the MC simulation was not modelled. For $p=1$, the measured cumulative profile shape for the sequential scan mode presents peaks, as $p=b / n T$, from the peaks presented in the cumulative profile. It can be inferred that the beam width $(a)$ is greater than the nominal beam width $(n T)$. This fact is supported by the analysis of the primary profiles measured with OSL ribbons. The results show that the over-beaming factor $a / n T$ were 1.05 , and 1.10 for $n T=20 \mathrm{~mm}$, and $n T=40 \mathrm{~mm}$, respectively. In the CT service mode, the over-beaming factor was 1.30 for $n T=40 \mathrm{~mm}$. Therefore, the CT service mode produces a beam width $30 \%$ higher than the nominal beam width. Since the calibration of the simulated dose profiles were performed using the experimental values of the corresponding air-Kerma length product measured by the ion chamber of $10 \mathrm{~cm}$ length, the CT dose metrics derived from the cumulative profiles should not be greatly affected by the no incorporation of the over-beaming factor in the MC simulation.

Equilibrium dose $\left(D_{e q}\right)$, Dose Line Integral ( $\left.D L I\right)$, Equilibrium dose-pitch product $\left(p x D_{e q}\right)$, the approach to equilibrium function $(H(L))$, and the equilibrium scanning length $\left(L_{e q}\right)$ from simulation were determined. A comprehensive study of these new dosimetric indexes was performed for the head and body helical protocols with $n T=20 \mathrm{~mm}$ and $n T=40 \mathrm{~mm}$ that are the most common values in the clinical services taking as a reference for the present work. The computation of the $D_{e q}$ by fitting the curve $D L I$ versus $L$ showed good accuracy and produce important data to be used in clinical services. In general terms, the clinical parameters such as, the pitch, beam intensity, and X-ray spectrum affect the $D_{e q}$ values. $D_{e q}$ values were used to divide the maximum dose at $\mathrm{z}=0$ of the cumulative profile to 
determine $H(L)$ which represent important characteristics of the dose distribution on CTDI phantom at central and peripheral axis. The approach to equilibrium function showed independence with the pitch values, but depends on the phantom axis. The $H(L)$ was used to determine the $L_{e q}$ by fitting the curve with an exponential model, as suggested by the analytical equation developed by Dixon and Boone (Dixon and Boone, 2010). $L_{e q}$ determined in this work for the $120 \mathrm{kV}$ X-ray spectrum were $30.1 \mathrm{~cm}$, and $28.1 \mathrm{~cm}$ at peripheral and central axes of the head phantom and $35.0 \mathrm{~cm}$ and $45.0 \mathrm{~cm}$ at peripheral and central axes of the body phantom.

The computation of the $p x D_{e q}$ put in evidence the limitations of the $C T D I_{100}$ method to determine the dose delivered by a CT scanner. Therefore, quantities derived from the $C T D I_{100}$ propagates that limitation. In this study, it was demonstrated that the efficiency of the $C T D I_{100}$ measurement in MDCT scanners is $80.6 \%$ and $87.8 \%$ at central and peripheral axes, respectively, for head phantom with $n T=20 \mathrm{~mm}, 56.5 \%$ and $86.2 \%$ at central and peripheral axes, respectively, for body phantom with $n T=40 \mathrm{~mm}$, all data for $120 \mathrm{kV}$ X-ray spectrum. 
Appendix A: Experimental evaluation for the $C T D I_{100}$ in GE Discovery CT750 HD

\section{- Head CTDI phantom}

Table 26. CTDI $I_{100}$ values at central and peripheral axes, for tube voltages of $80,100,120$, and $140 \mathrm{kV}$.

\begin{tabular}{lllllcc}
\hline $\begin{array}{c}\text { Voltage } \\
(\mathbf{k V})\end{array}$ & Position & \multicolumn{2}{c}{$\begin{array}{c}\text { Reading } \\
(\mathbf{m G y} / \mathbf{1 0 0 m A s})\end{array}$} & $\begin{array}{c}\text { CTDI } \\
\text { (mGy/100mAs) }\end{array}$ & $\begin{array}{c}\text { Relative } \\
\text { Uncertainty }(\%)\end{array}$ \\
\hline \multirow{4}{*}{80} & Central & 0.931 & 0.932 & 0.932 & 9.32 & 0.01 \\
& Peripheral - 1 & 1.162 & 1.161 & 1.162 & 11.62 & 0.02 \\
& Peripheral - 2 & 1.007 & 1.010 & 1.009 & 10.08 & 0.09 \\
& Peripheral - 3 & 0.830 & 0.831 & 0.830 & 8.30 & 0.03 \\
& Peripheral - 4 & 1.016 & 1.017 & 1.017 & 10.17 & 0.03 \\
\hline \multirow{3}{*}{100} & Central & 1.749 & 1.749 & 1.749 & 17.49 & 0.0 \\
& Peripheral - 1 & 2.052 & 2.052 & 2.052 & 20.52 & 0.0 \\
& Peripheral - 2 & 1.810 & 1.809 & 1.810 & 18.10 & 0.02 \\
& Peripheral - 3 & 1.549 & 1.546 & 1.548 & 15.48 & 0.06 \\
& Peripheral - 4 & 1.825 & 1.822 & 1.824 & 18.24 & 0.05 \\
\hline \multirow{3}{*}{120} & Central & 2.739 & 2.744 & 2.742 & 27.42 & 0.05 \\
& Peripheral - 1 & 3.110 & 3.110 & 3.110 & 31.10 & 0.0 \\
& Peripheral - 2 & 2.764 & 2.765 & 2.765 & 27.65 & 0.01 \\
& Peripheral - 3 & 2.409 & 2.410 & 2.410 & 24.10 & 0.01 \\
& Peripheral - 4 & 2.785 & 2.784 & 2.785 & 27.85 & 0.01 \\
\hline \multirow{3}{*}{140} & Central & 3.883 & 3.881 & 3.882 & 38.82 & 0.01 \\
& Peripheral - 1 & 4.314 & 4.315 & 4.315 & 43.15 & 0.01 \\
& Peripheral - 2 & 3.864 & 3.863 & 3.864 & 38.64 & 0.01 \\
& Peripheral - 3 & 3.388 & 3.398 & 3.393 & 33.93 & 0.09 \\
& Peripheral - 4 & 3.882 & 3.886 & 3.884 & 38.84 & 0.03 \\
\hline
\end{tabular}




\section{- Body CTDI phantom}

Table 27. $C T D I_{100}$ values at central and peripheral axes, for tube voltages of $80,100,120$, and

\begin{tabular}{llllcc}
$\begin{array}{c}\text { Voltage } \\
(\mathbf{k V})\end{array}$ & Position & \multicolumn{2}{c}{$\begin{array}{c}\text { Reading } \\
\text { (mGy/100mAs) }\end{array}$} & $\begin{array}{c}\text { CTDI } \\
\text { (mGy/100mAs) }\end{array}$ & $\begin{array}{c}\text { Relative } \\
\text { Uncertainty }(\%)\end{array}$ \\
& Central & 0.204 & 0.202 & 2.03 & 0.4 \\
$\mathbf{8 0}$ & Peripheral - 1 & 0.633 & 0.664 & 6.48 & 2.4 \\
& Peripheral - 2 & 0.580 & 0.580 & 5.80 & 0.0 \\
& Peripheral - 3 & 0.460 & 0.460 & 4.60 & 0.04 \\
& Peripheral - 4 & 0.580 & 0.579 & 5.80 & 0.08 \\
& Central & 0.468 & 0.466 & 4.67 & 0.2 \\
$\mathbf{1 0 0}$ & Peripheral - 1 & 1.198 & 1.198 & 11.98 & 0.0 \\
& Peripheral - 2 & 1.103 & 1.102 & 11.03 & 0.05 \\
& Peripheral - 3 & 0.902 & 0.902 & 9.02 & 0.0 \\
& Peripheral - 4 & 1.097 & 1.099 & 10.98 & 0.09 \\
& Central & 0.802 & 0.802 & 8.02 & 0.02 \\
$\mathbf{1 2 0}$ & Peripheral - 1 & 1.886 & 1.888 & 18.87 & 0.05 \\
& Peripheral - 2 & 1.748 & 1.750 & 17.49 & 0.06 \\
& Peripheral - 3 & 1.456 & 1.455 & 14.56 & 0.03 \\
& Peripheral - 4 & 1.734 & 1.734 & 17.34 & 0.0 \\
& Central & 1.211 & 1.209 & 12.10 & 0.08 \\
& Peripheral - 1 & 2.691 & 2.695 & 26.93 & 0.07 \\
$\mathbf{1 4 0}$ & Peripheral - 2 & 2.504 & 2.503 & 25.04 & 0.02 \\
& Peripheral - 3 & 2.107 & 2.106 & 21.07 & 0.02 \\
& Peripheral - 4 & 2.482 & 2.480 & 24.81 & 0.04
\end{tabular}


Appendix B: Dose Profiles Comparison
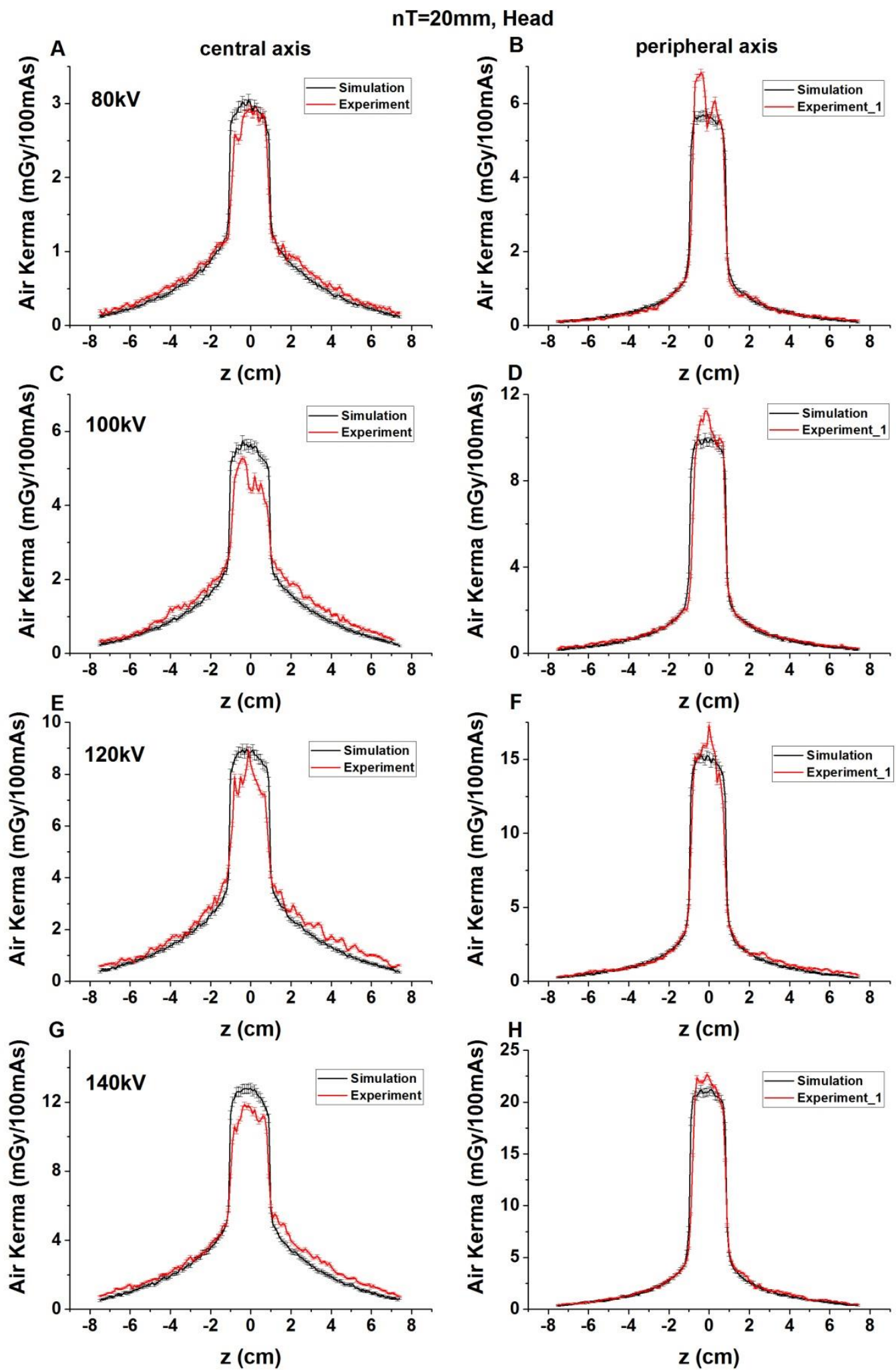

Figure 87. Simulated and measured dose profile comparison for Head CTDI phantom at nT=20 $\mathrm{mm}$. 


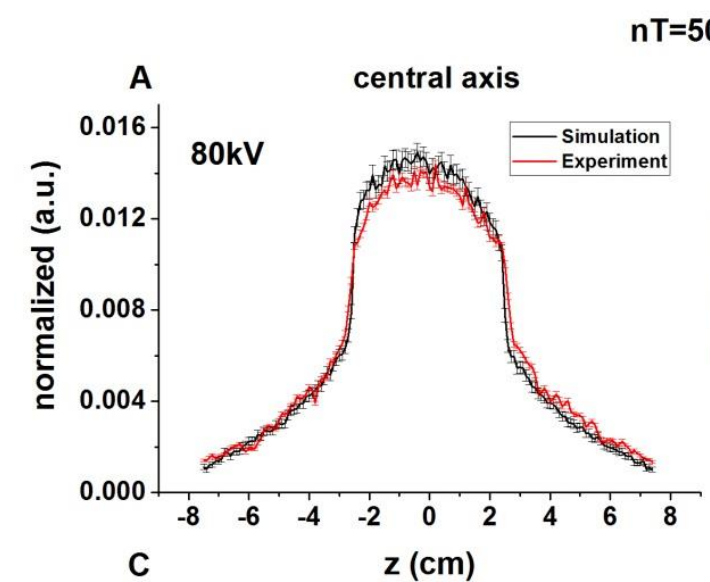

$\mathrm{nT}=50 \mathrm{~mm}$, Head
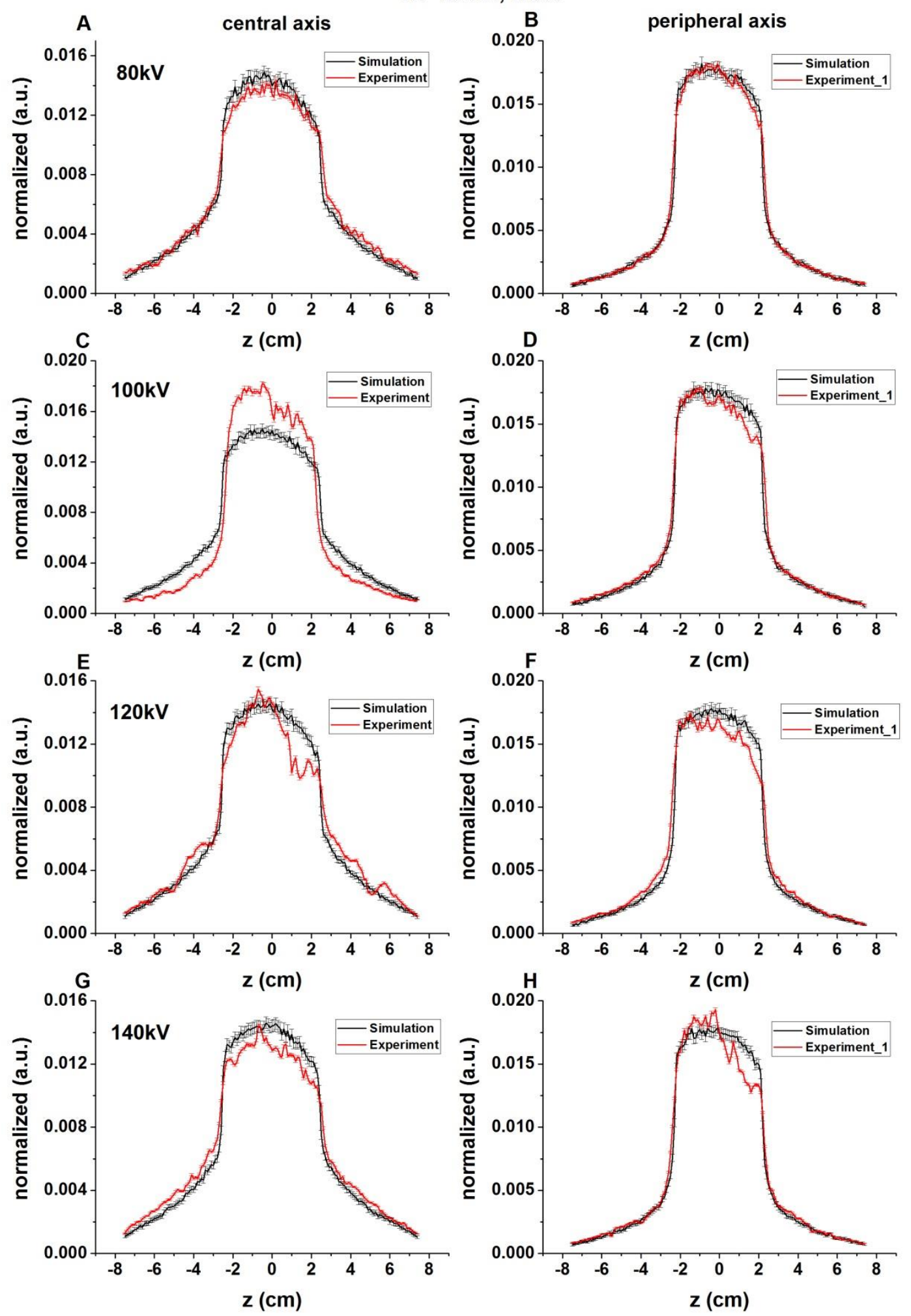

Figure 88. Simulated and measured dose profile comparison for Head CTDI phantom at nT=50mm. 


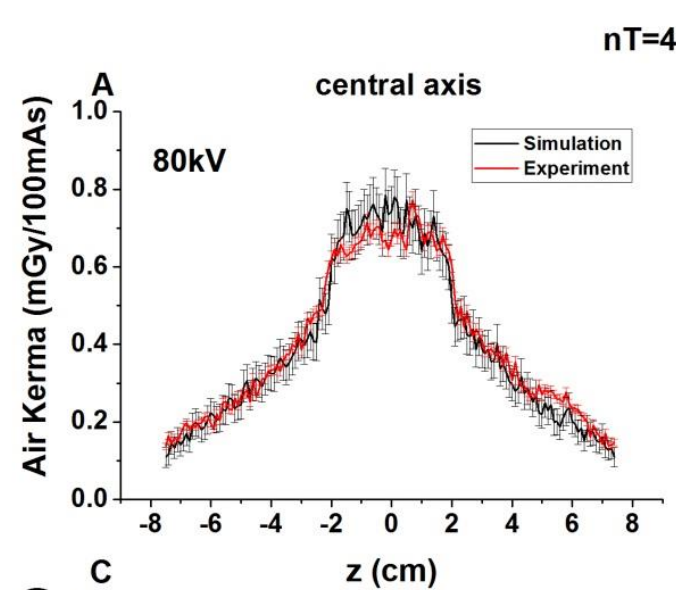

$\mathrm{nT}=40 \mathrm{~mm}$, Body
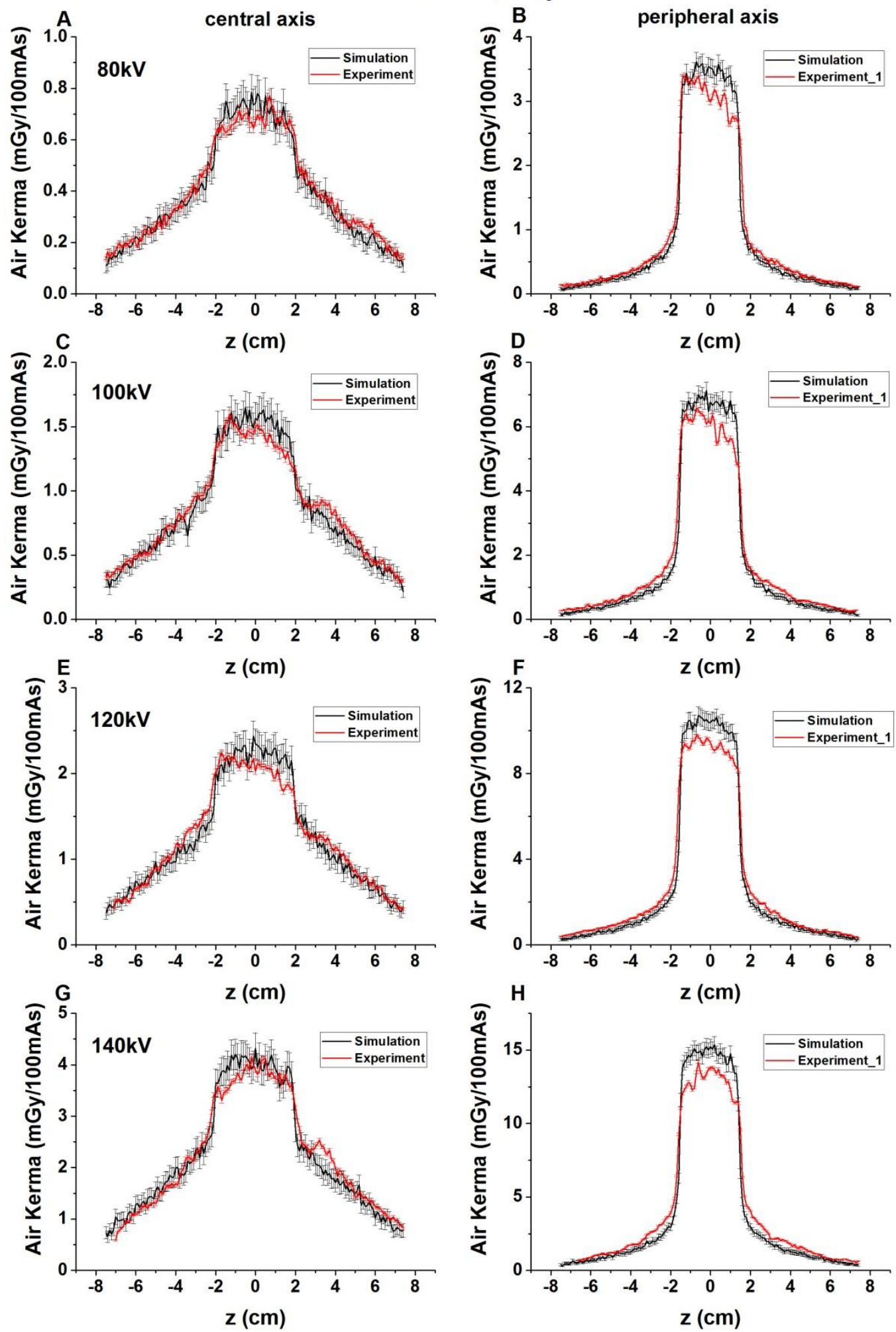

Figure 89. Simulated and measured dose profile comparison for Bead CTDI phantom at nT=40mm. 


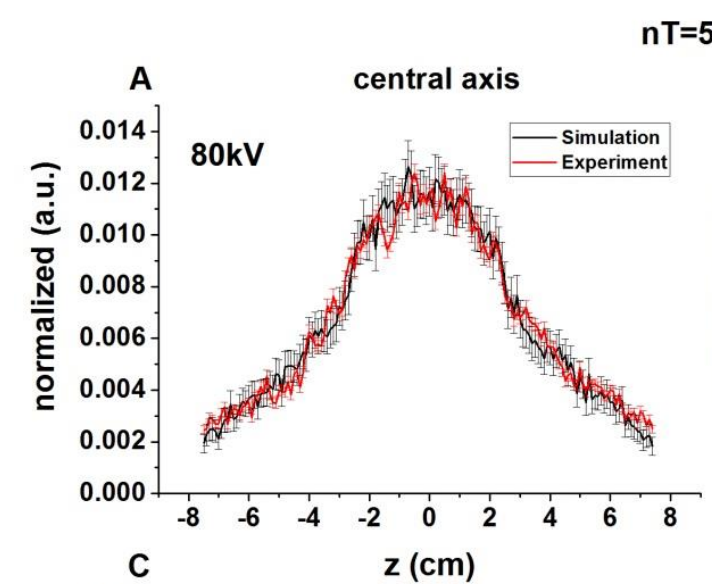

$\mathrm{T}=50 \mathrm{~mm}$, Body
$B$
0.025
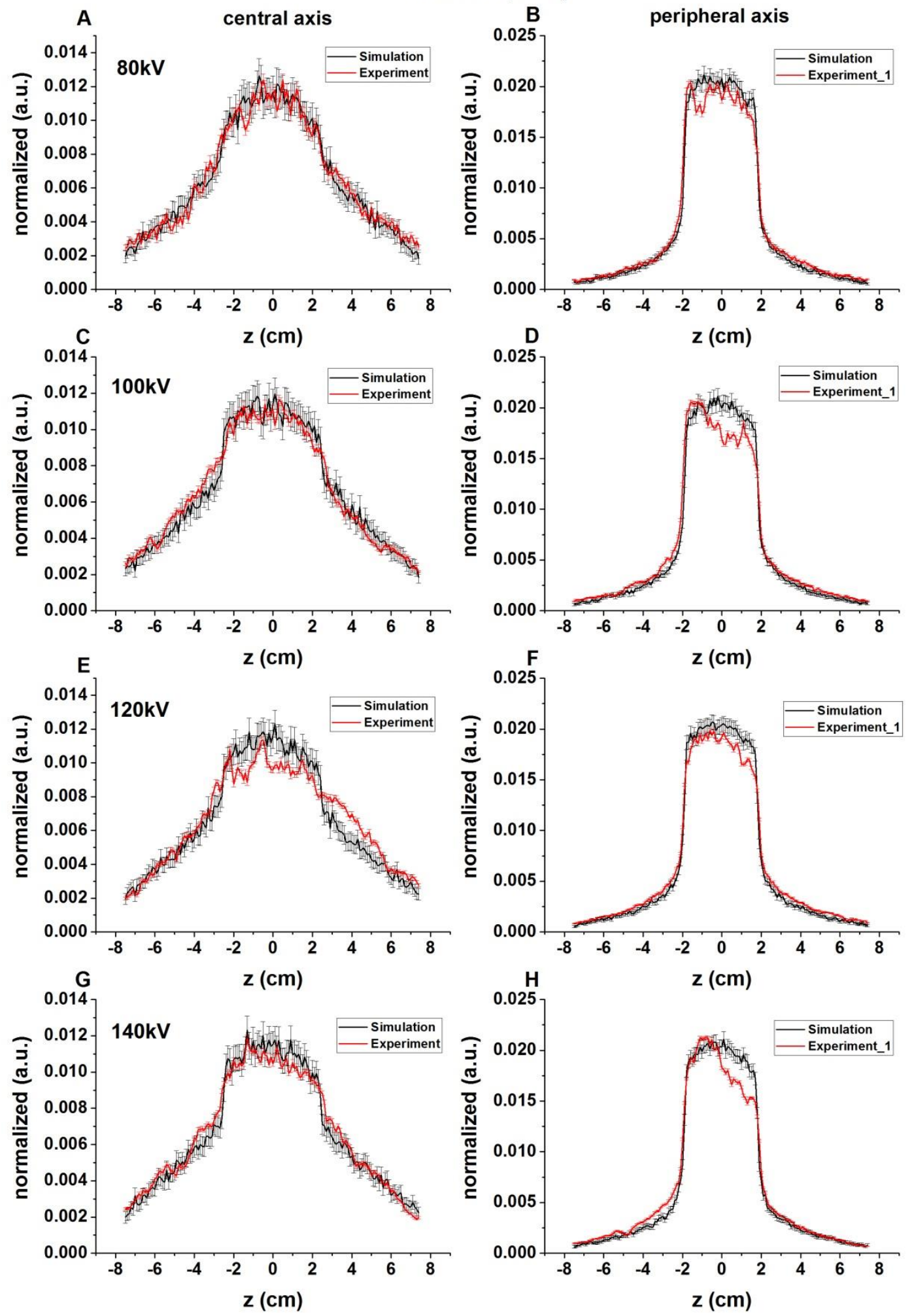

Figure 90. Simulated and measured dose profile comparison for Bead CTDI phantom at nT=40mm. 
Appendix C: Cumulative Dose Profiles from MC simulation.

- Cumulated profiles for head phantom
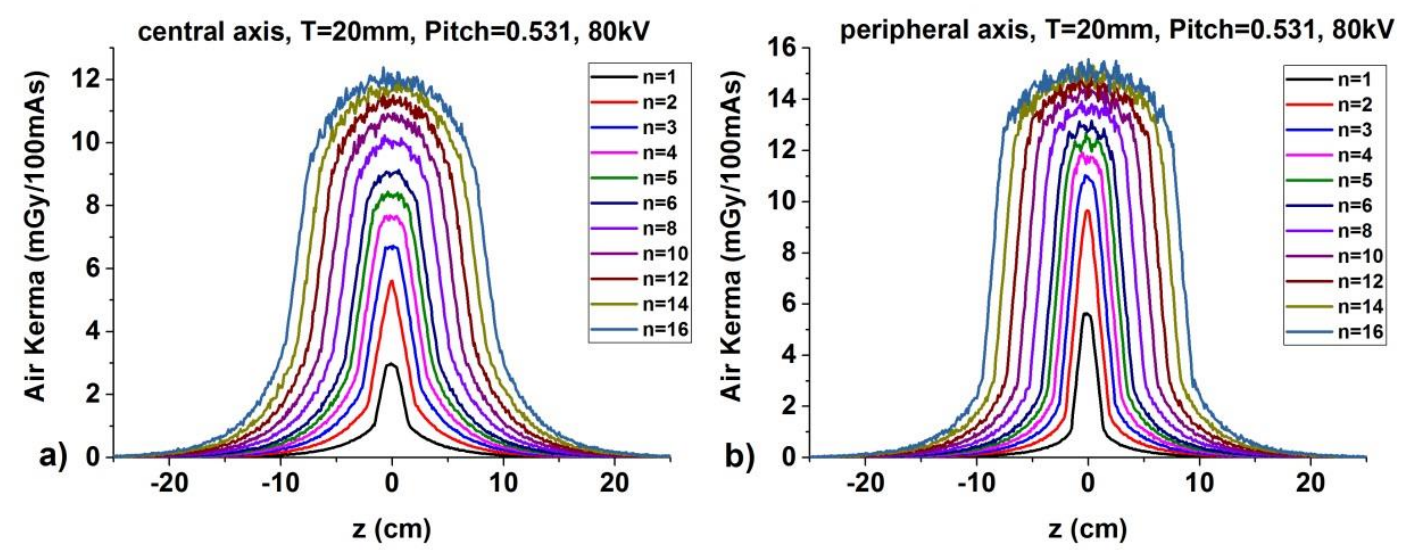

Figure 91. a) Central and b) peripheral cumulated dose profile in head CTDI phantom for helical scan with pitch $=\mathbf{0 . 5 3 1}$.
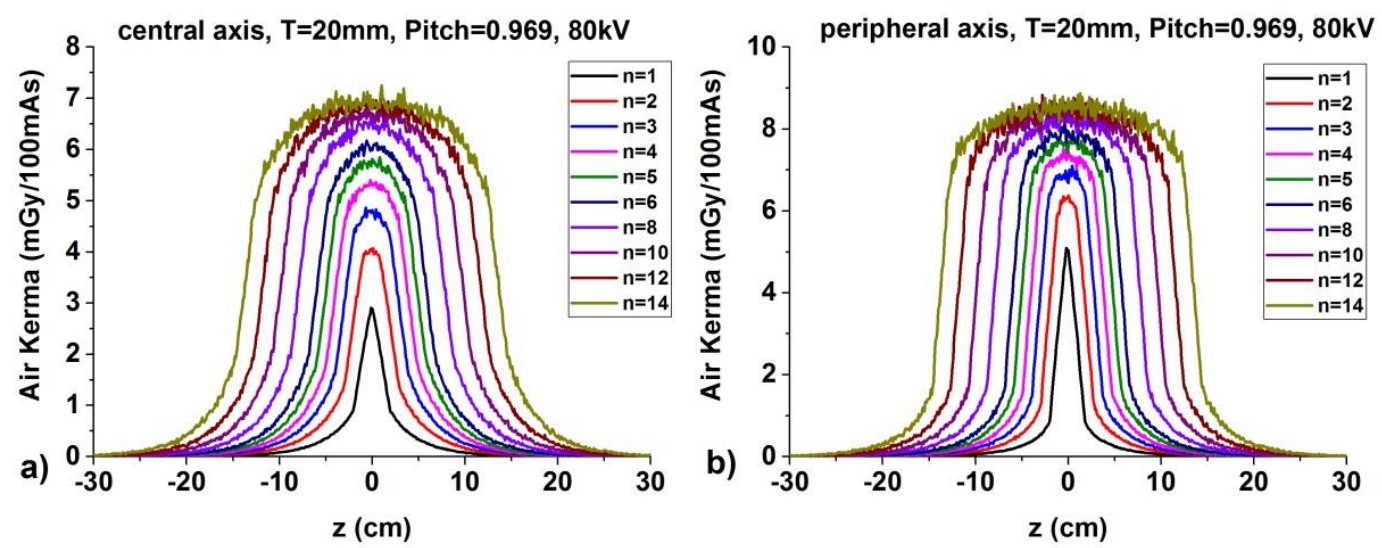

Figure 92. a) Central and b) peripheral cumulated dose profile in head CTDI phantom for helical scan with pitch=0.969. 

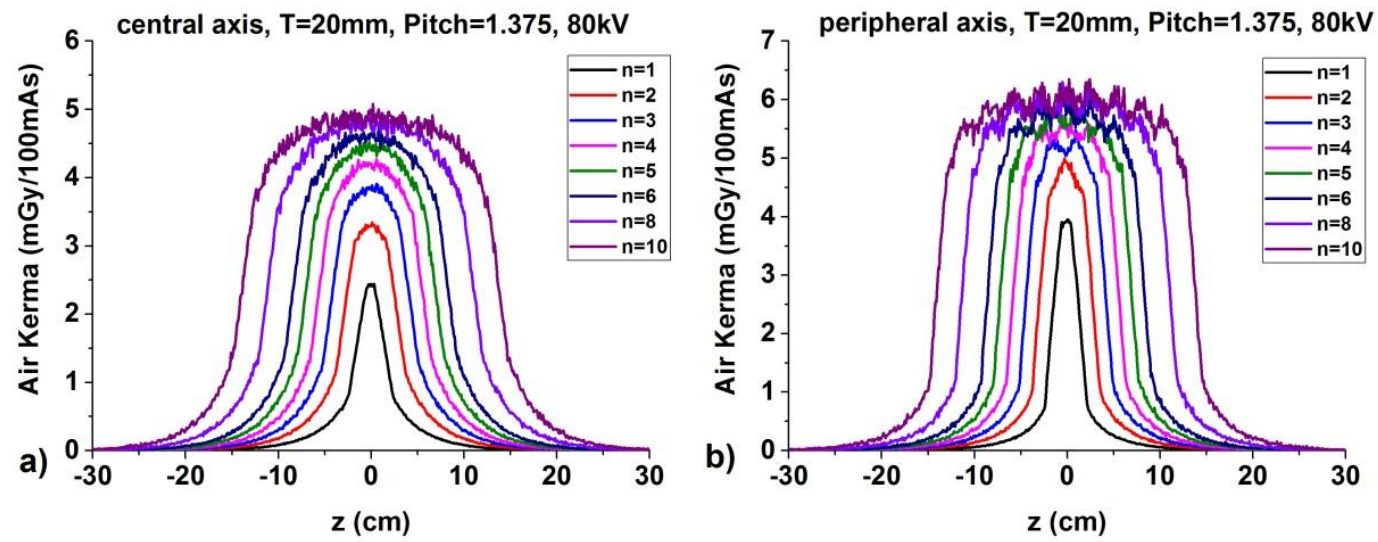

Figure 93. a) Central and b) peripheral cumulated dose profile in head CTDI phantom for helical scan with pitch=1.375.
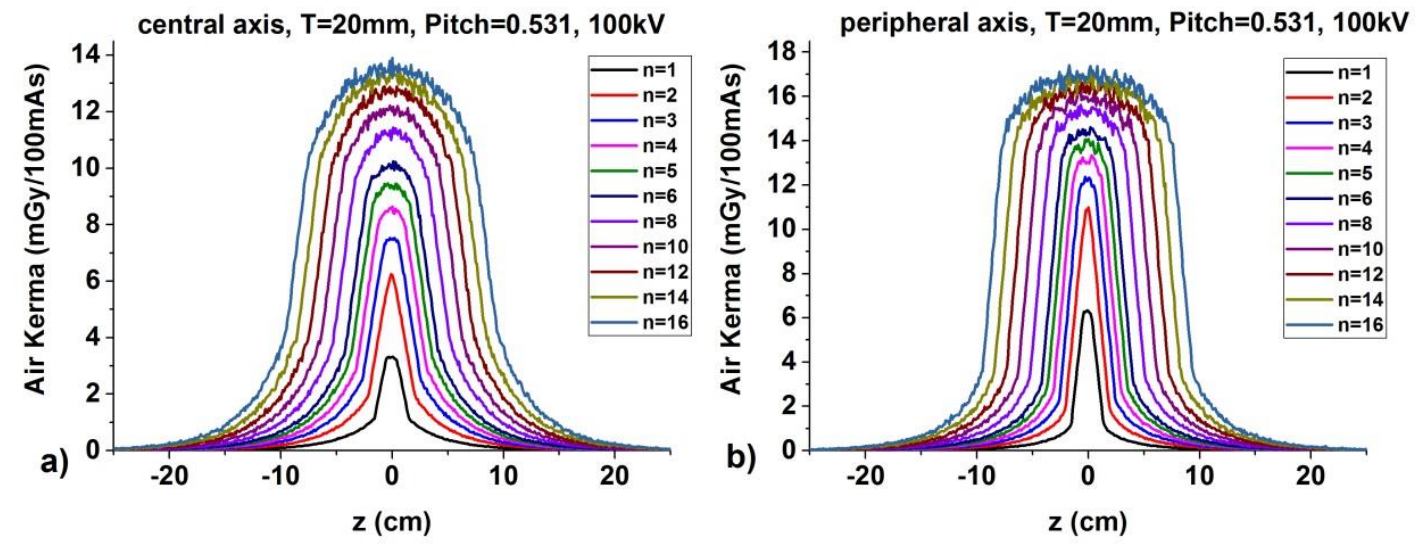

Figure 94. a) Central and b) peripheral cumulated dose profile in head CTDI phantom for helical scan with pitch=0.531.
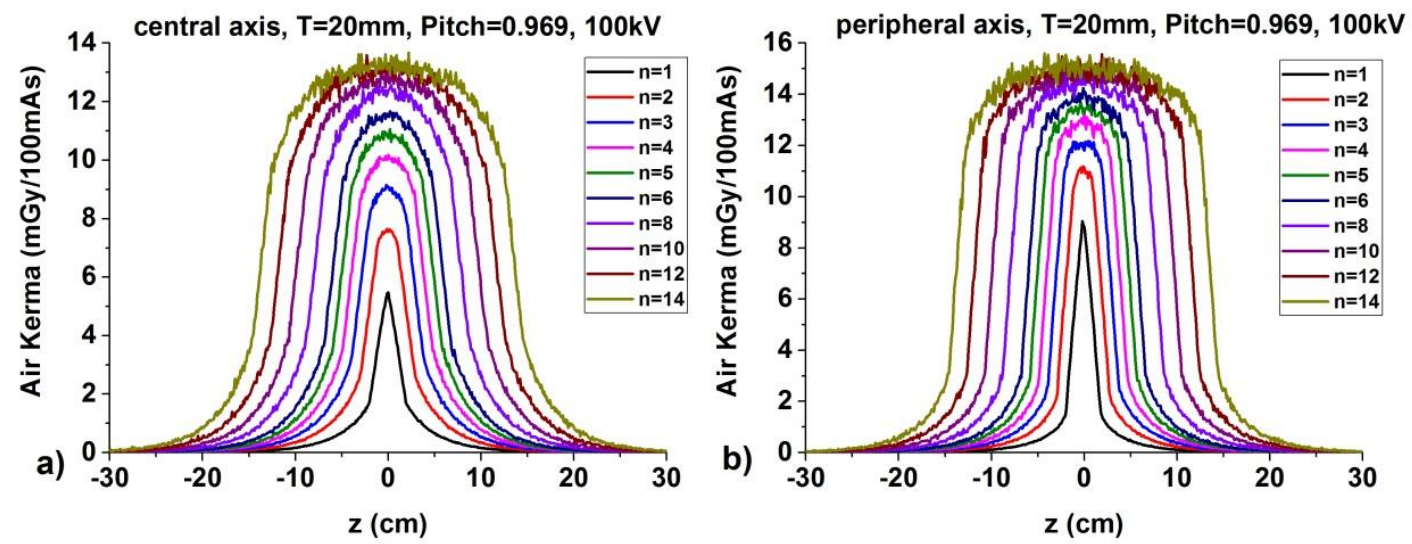

Figure 95. a) Central and b) peripheral cumulated dose profile in head CTDI phantom for helical scan with pitch=0.969. 

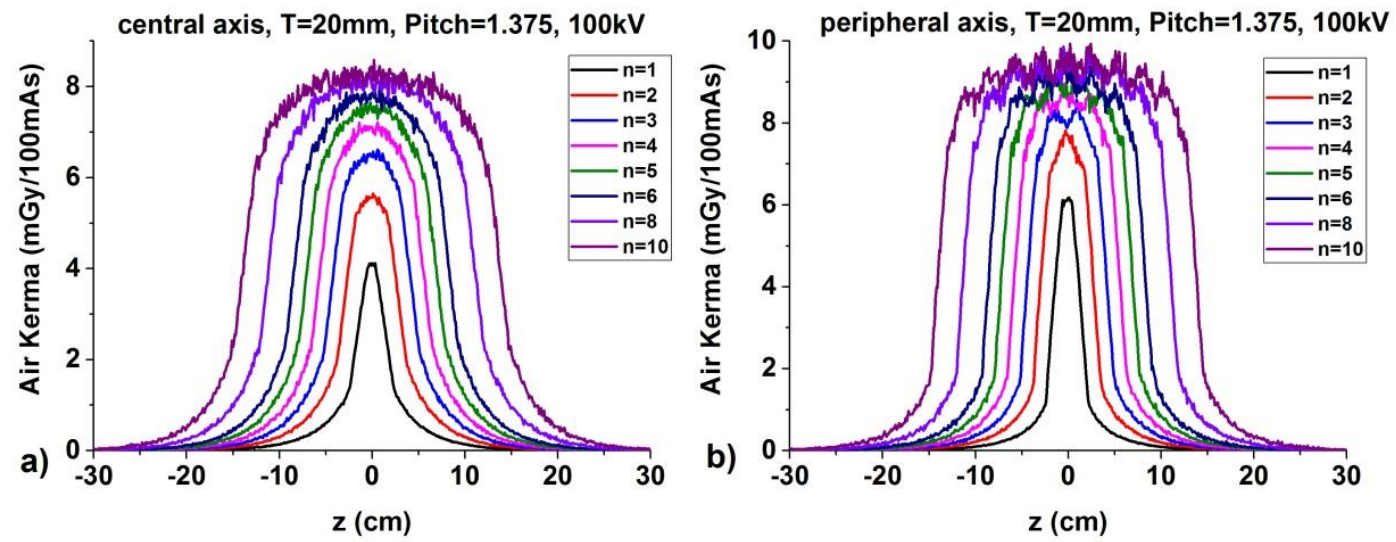

Figure 96. a) Central and b) peripheral cumulated dose profile in head CTDI phantom for helical scan with pitch=1.375.
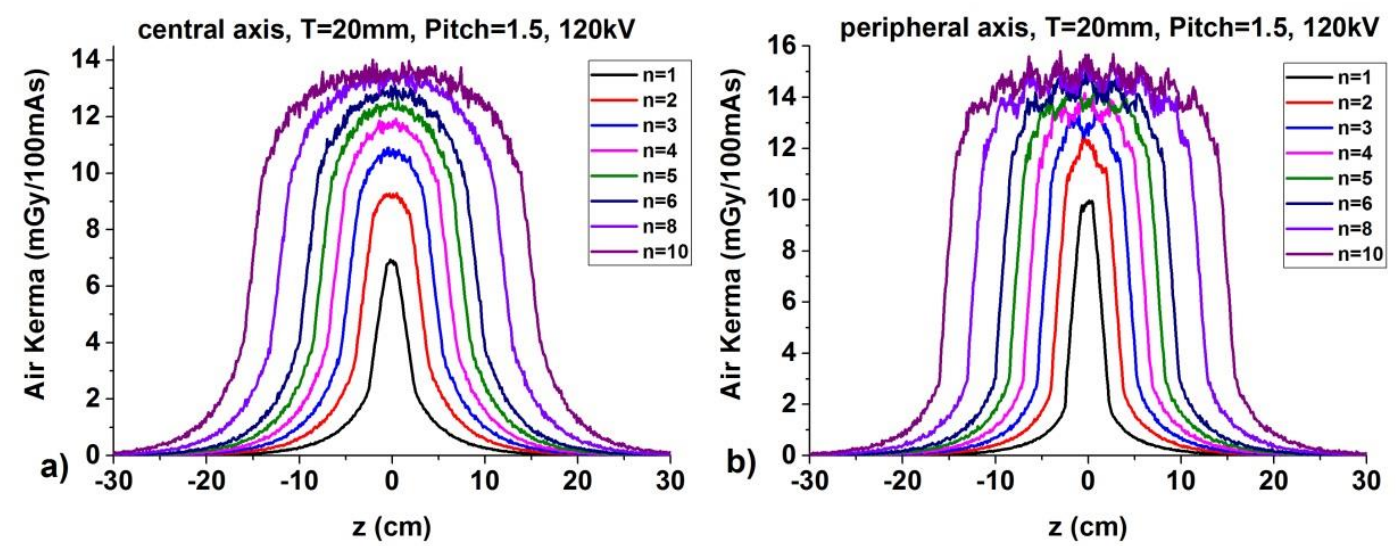

Figure 97. a) Central and b) peripheral cumulated dose profile in head CTDI phantom for helical scan with pitch=1.5.
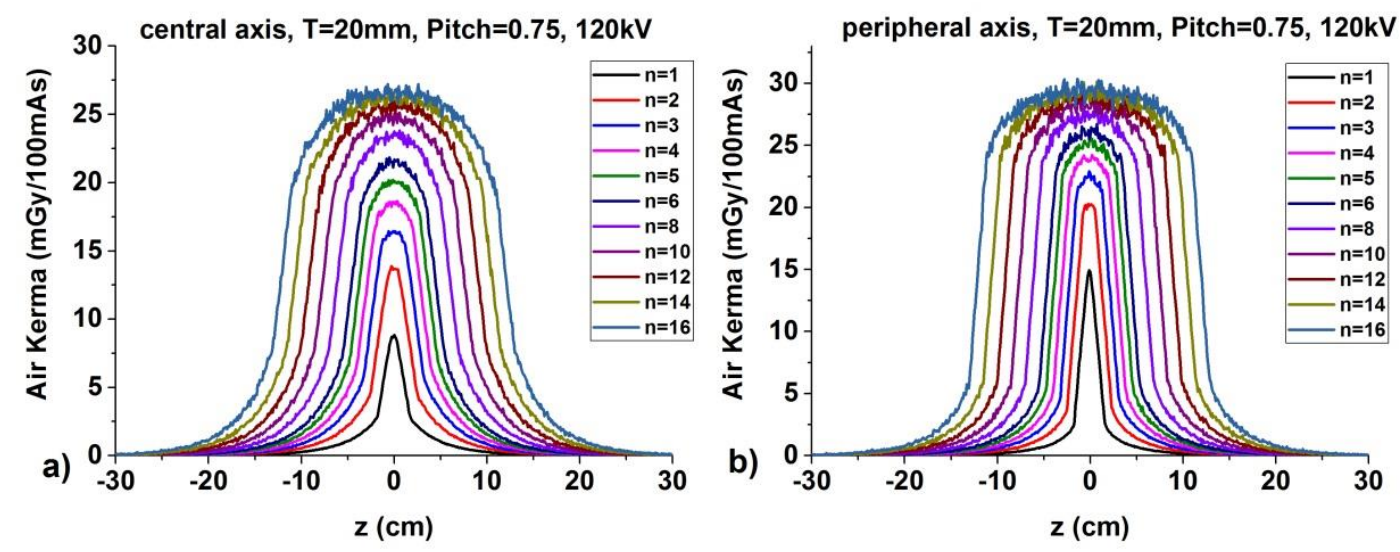

Figure 98. a) Central and b) peripheral cumulated dose profile in head CTDI phantom for helical scan with pitch $=0.75$. 

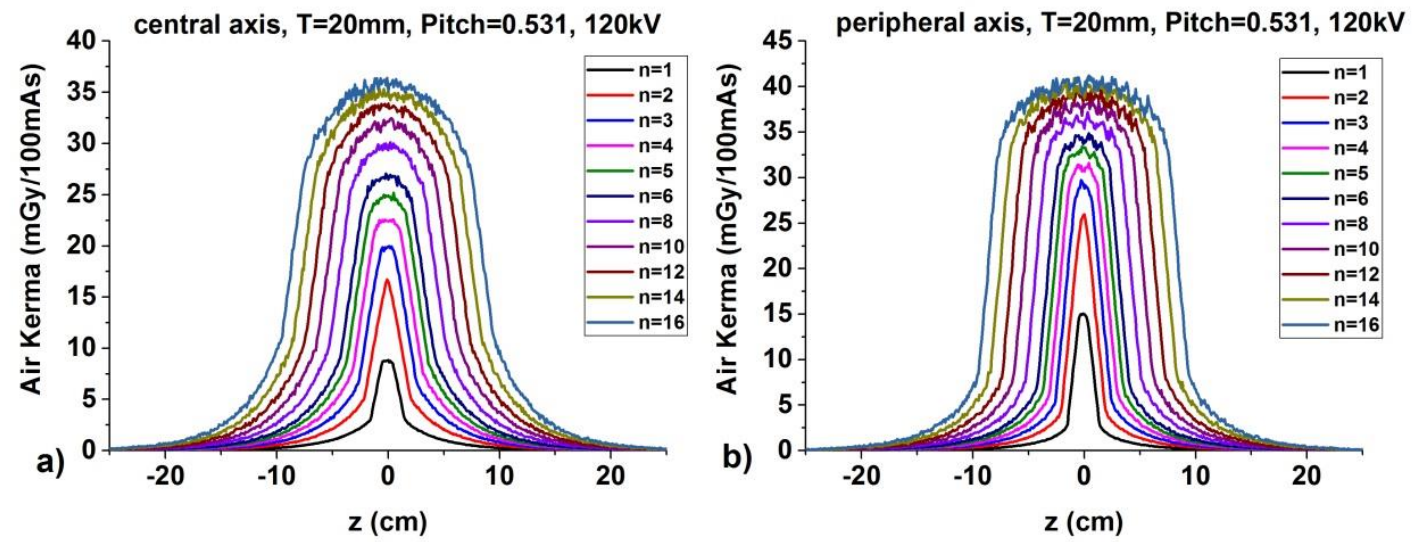

Figure 99. a) Central and b) peripheral cumulated dose profile in head CTDI phantom for helical scan with pitch $=\mathbf{0 . 5 3 1}$.
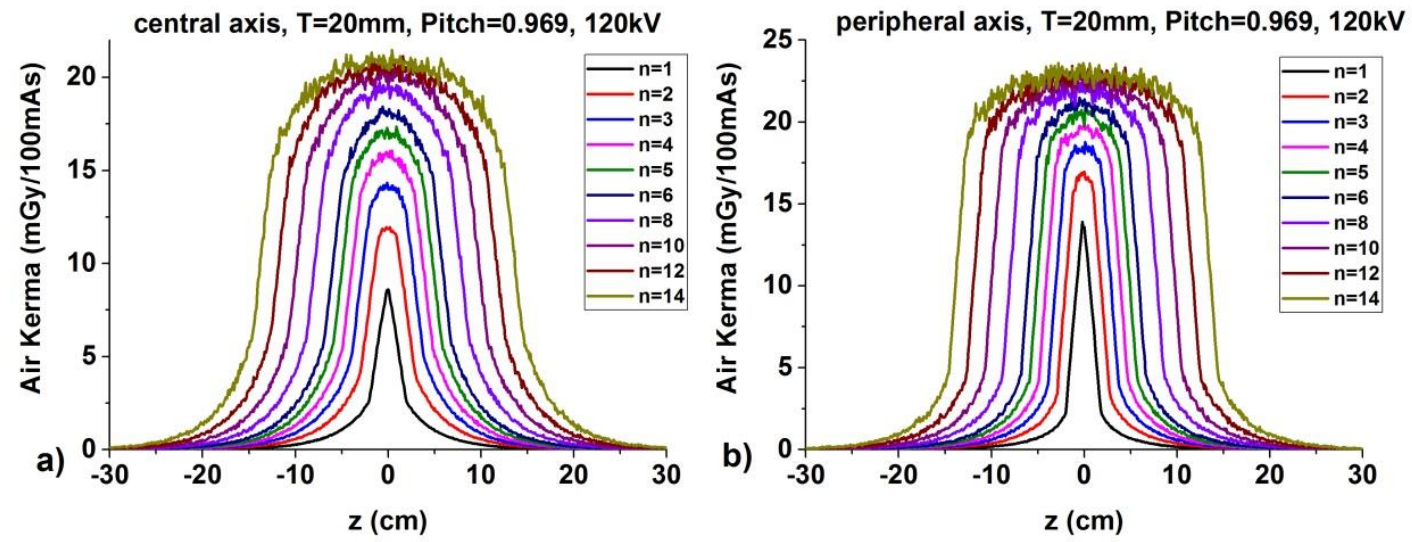

Figure 100. a) Central and b) peripheral cumulated dose profile in head CTDI phantom for helical scan with pitch=0.969.
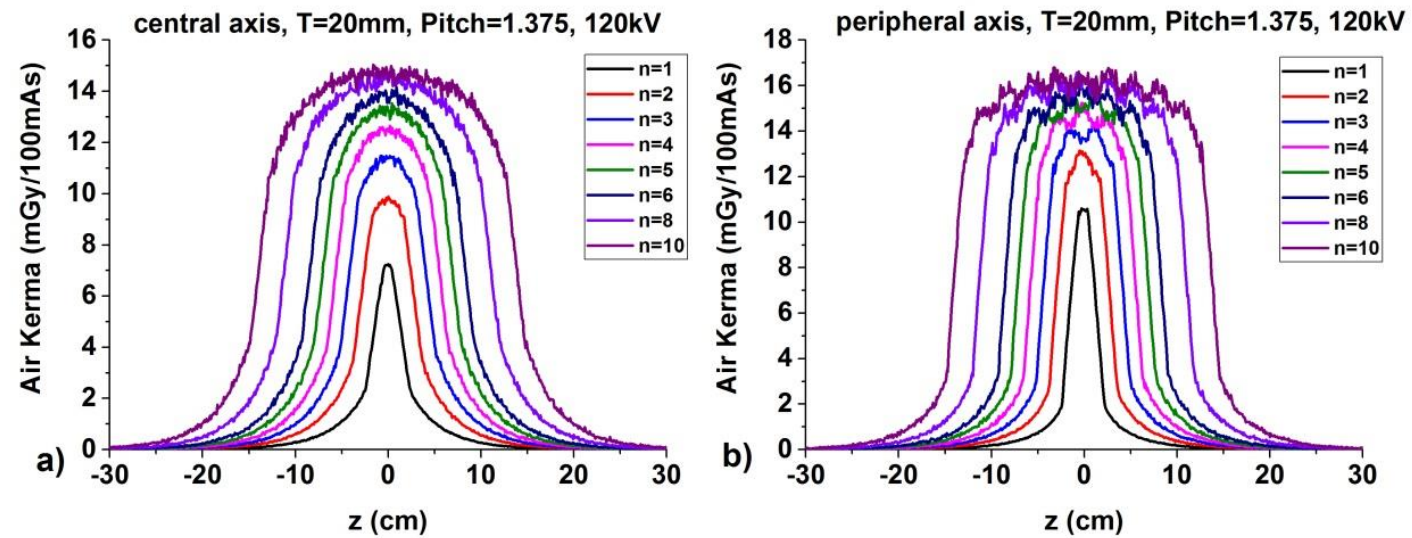

Figure 101. a) Central and b) peripheral cumulated dose profile in head CTDI phantom for helical scan with pitch=1.375. 

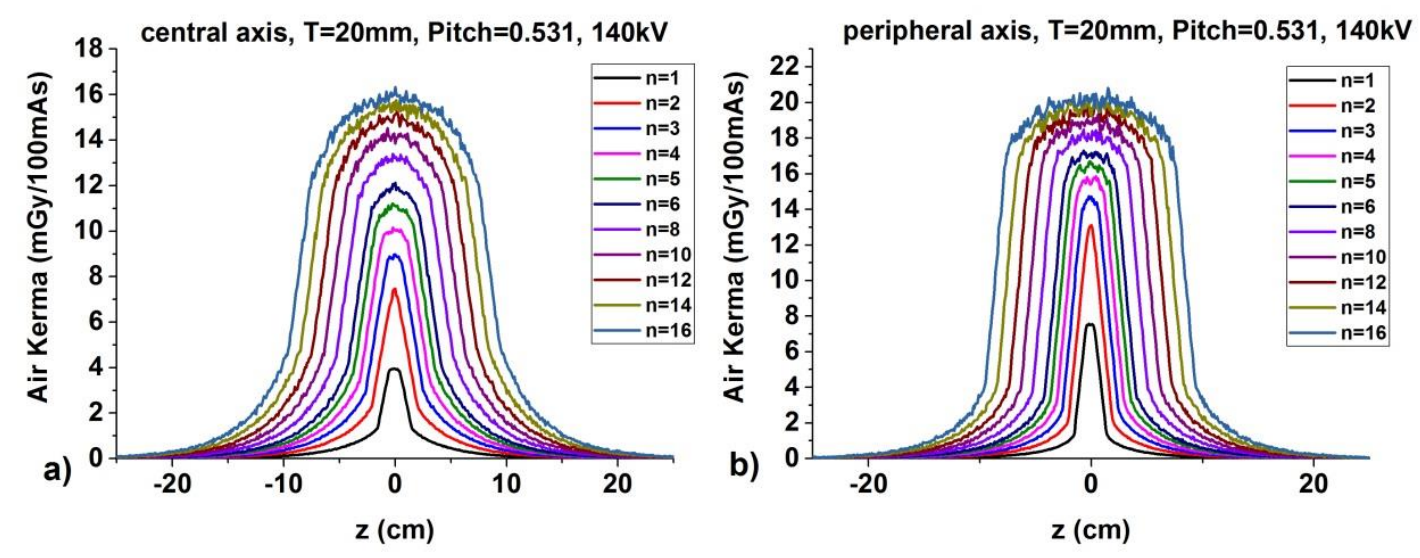

Figure 102. a) Central and b) peripheral cumulated dose profile in head CTDI phantom for helical scan with pitch $=\mathbf{0 . 5 3 1}$.
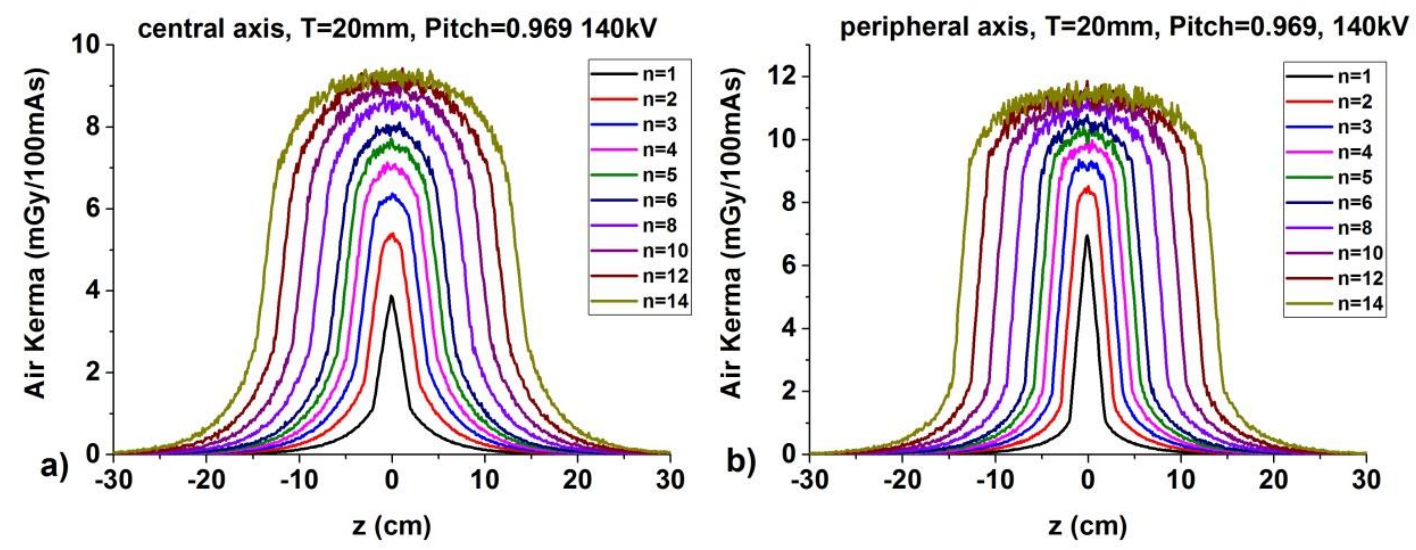

Figure 103. a) Central and b) peripheral cumulated dose profile in head CTDI phantom for helical scan with pitch=0.969.
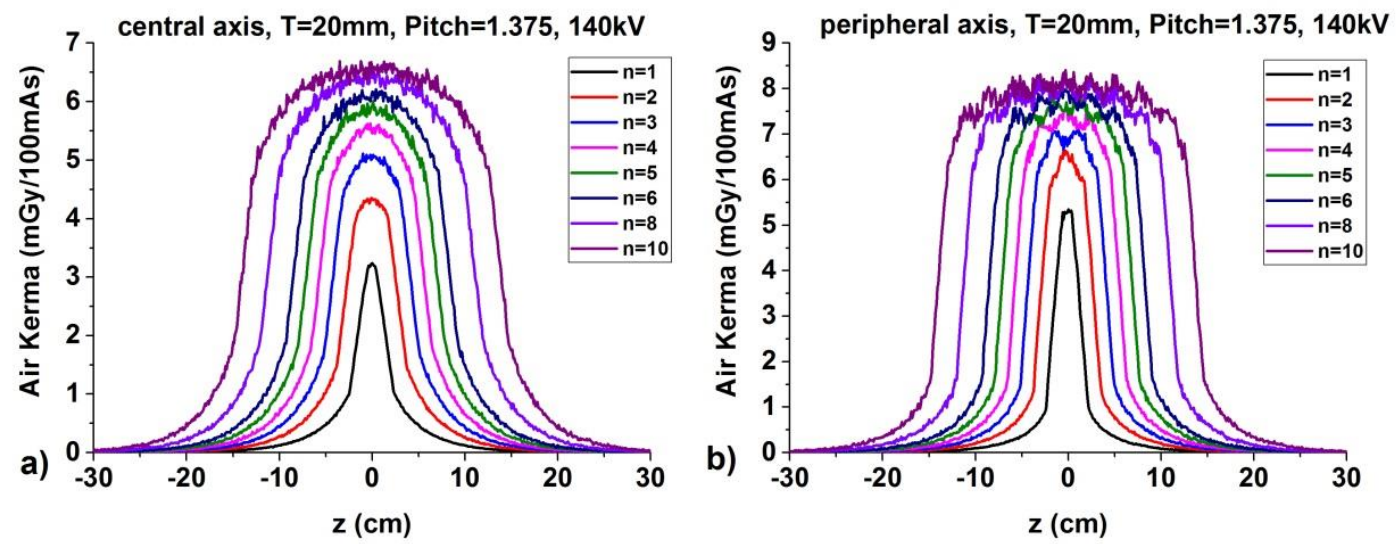

Figure 104. a) Central and b) peripheral cumulated dose profile in head CTDI phantom for helical scan with pitch=1.375. 


\section{- Cumulated dose profile for Body}
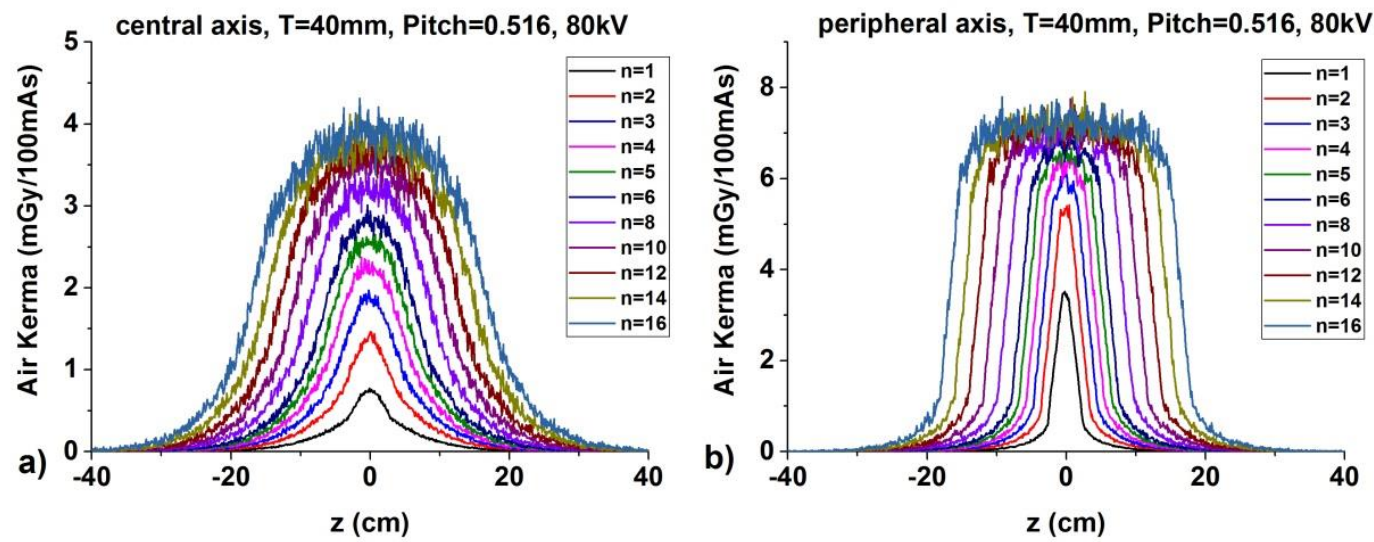

Figure 105. a) Central and b) peripheral cumulated dose profile in body CTDI phantom for helical scan with pitch $=\mathbf{0 . 5 1 6}$.
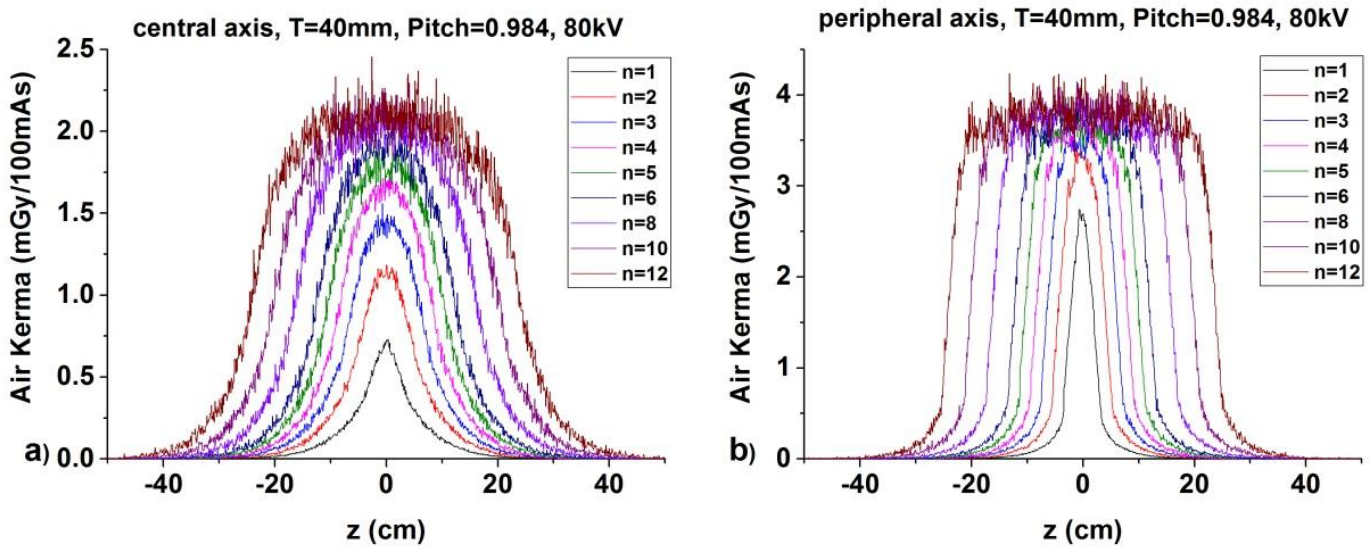

Figure 106. a) Central and b) peripheral cumulated dose profile in body CTDI phantom for helical scan with pitch=0.984.
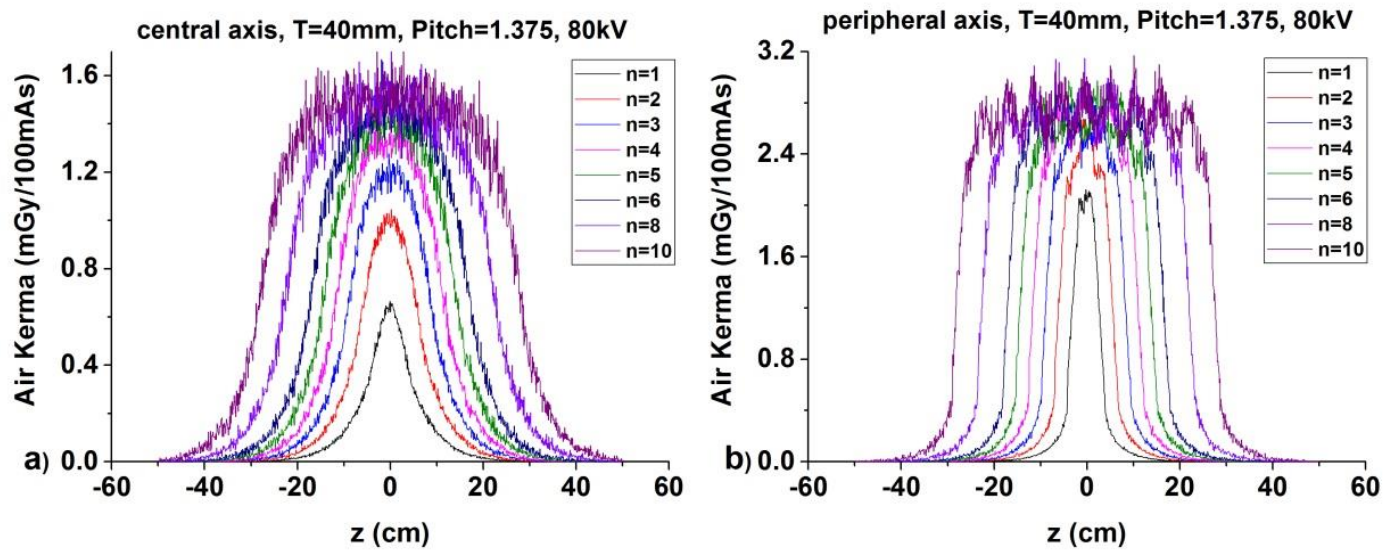

Figure 107. a) Central and b) peripheral cumulated dose profile in body CTDI phantom for helical scan with pitch=1.375. 

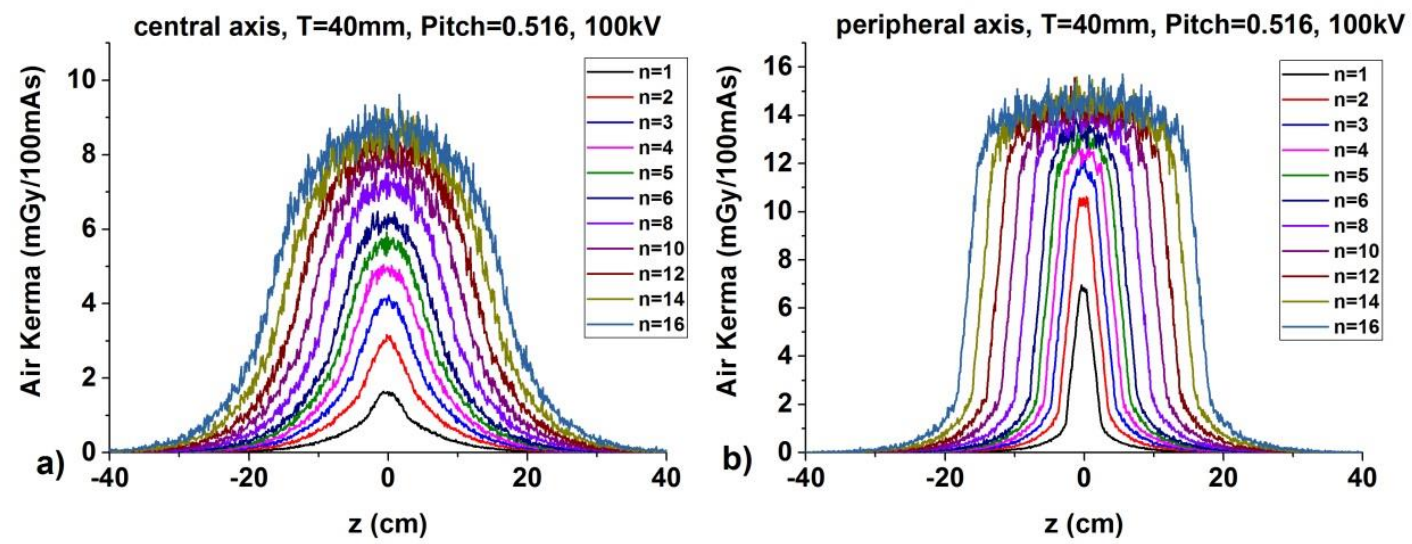

Figure 108. a) Central and b) peripheral cumulated dose profile in body CTDI phantom for helical scan with pitch $=0.516$.
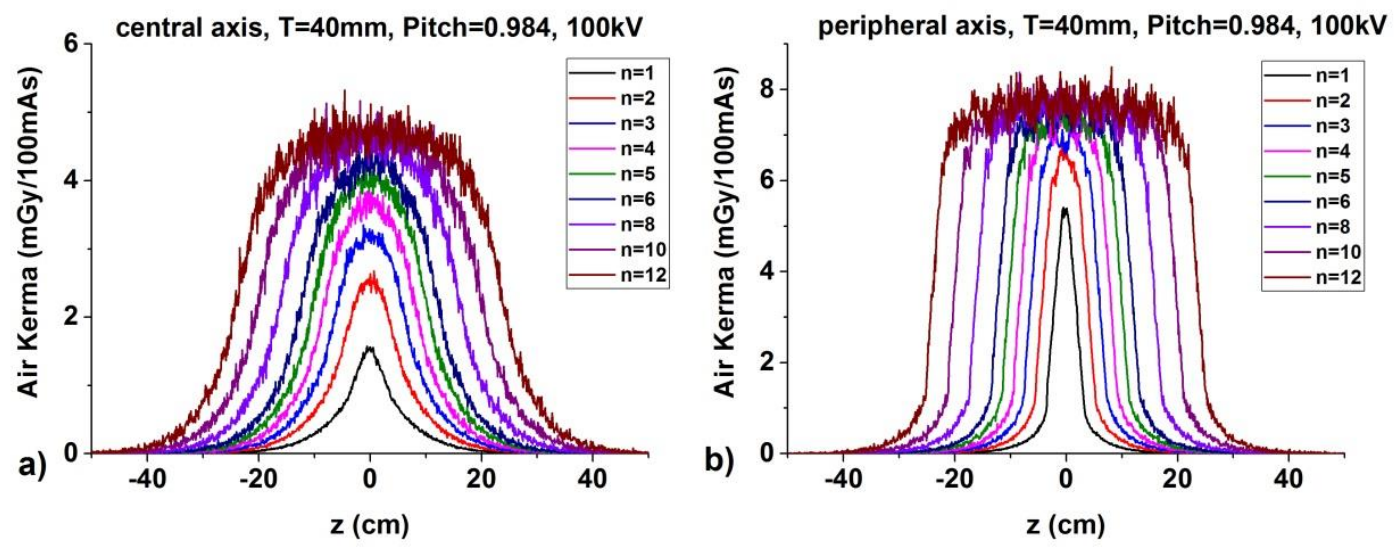

Figure 109. a) Central and b) peripheral cumulated dose profile in body CTDI phantom for helical scan with pitch=0.984.
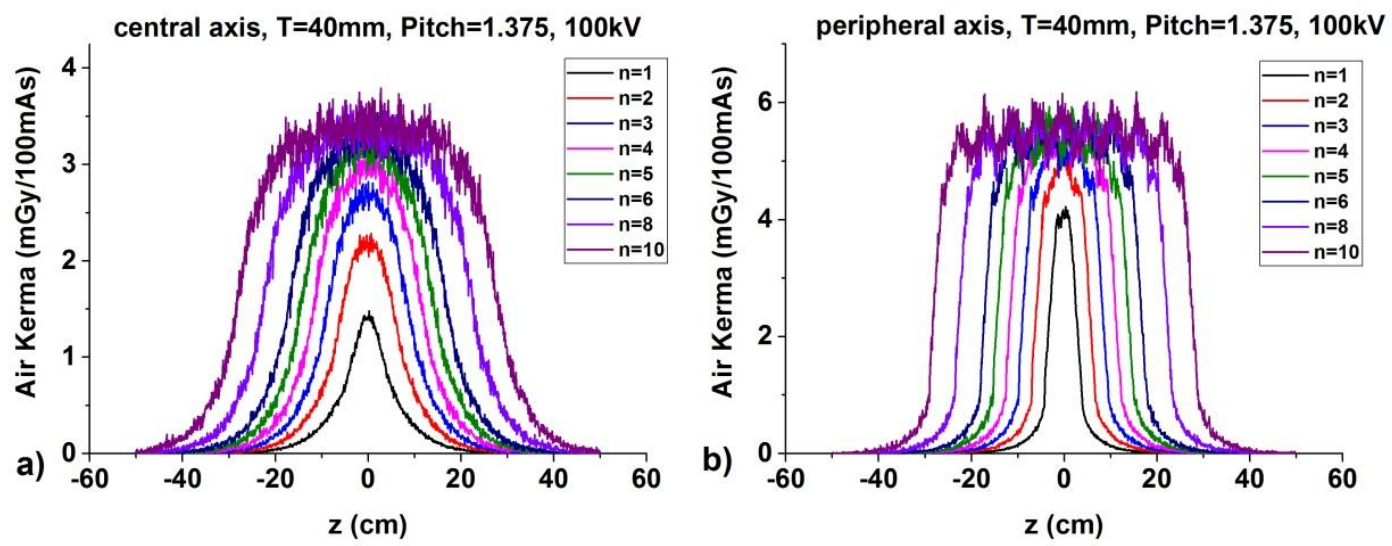

Figure 110. a) Central and b) peripheral cumulated dose profile in body CTDI phantom for helical scan with pitch $=1.375$. 

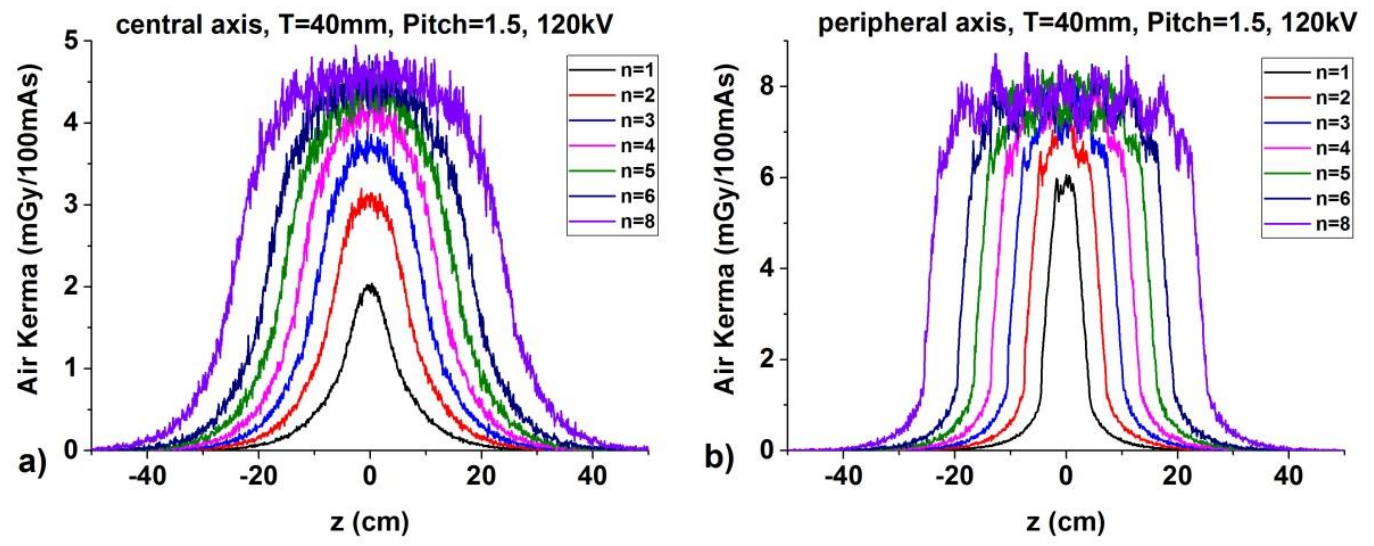

Figure 111. a) Central and b) peripheral cumulated dose profile in body CTDI phantom for helical scan with pitch=1.5.
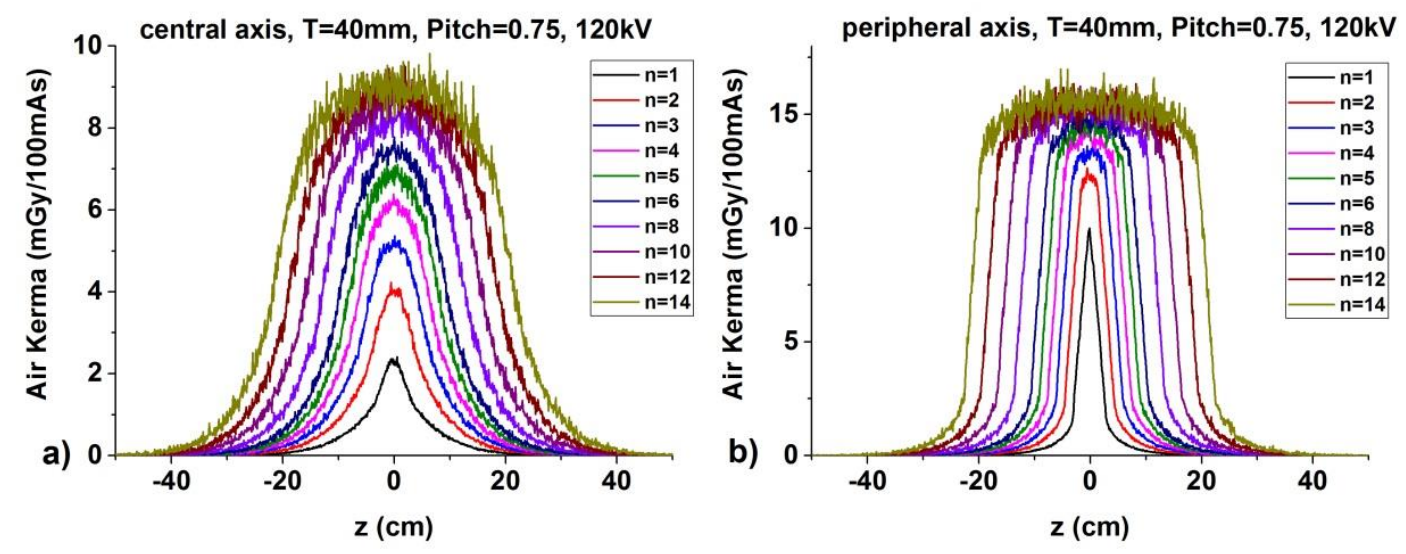

Figure 112. a) Central and b) peripheral cumulated dose profile in body CTDI phantom for helical scan with pitch=0.75.
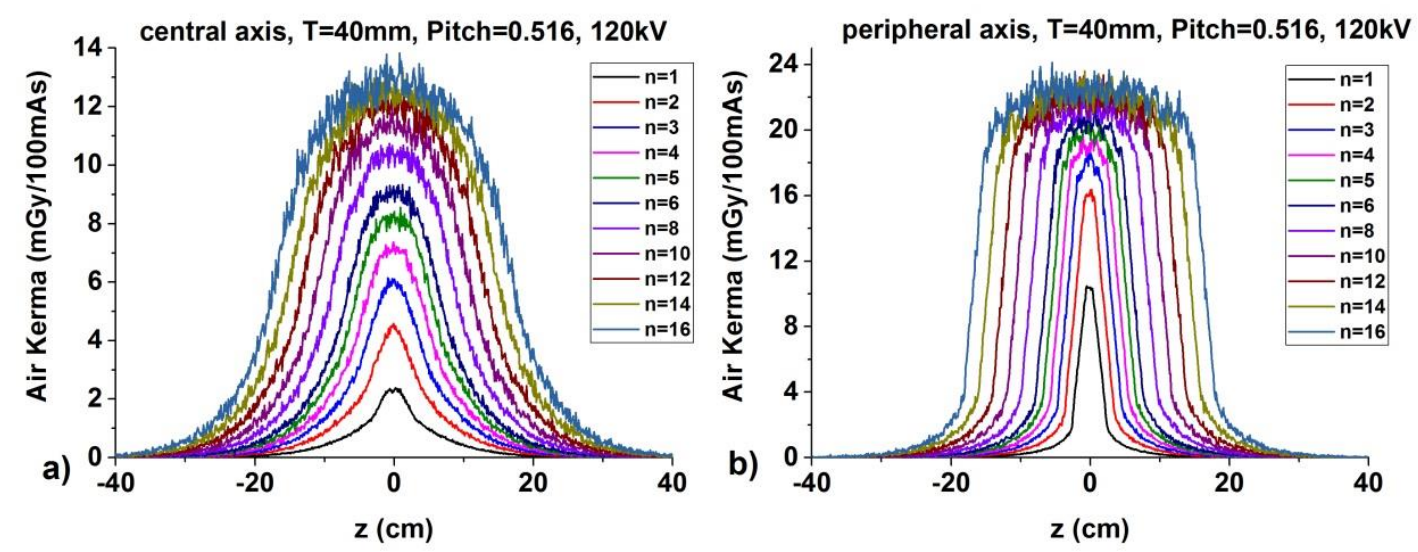

Figure 113. a) Central and b) peripheral cumulated dose profile in body CTDI phantom for helical scan with pitch=0.516. 

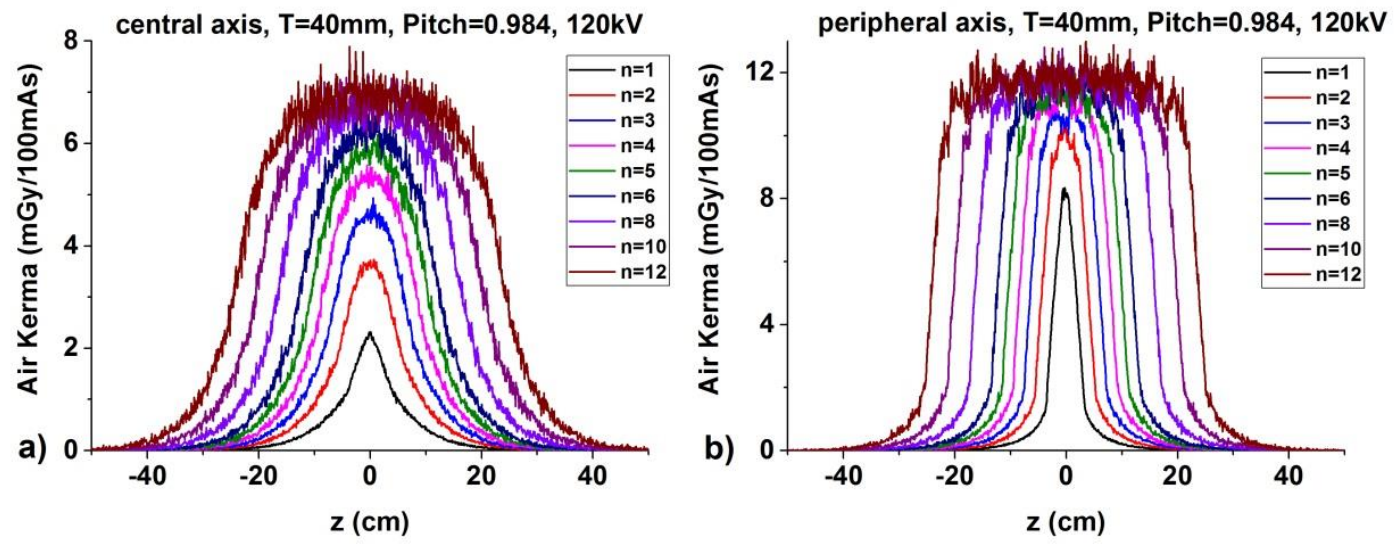

Figure 114. a) Central and b) peripheral cumulated dose profile in body CTDI phantom for helical scan with pitch $=\mathbf{0 . 9 8 4}$.
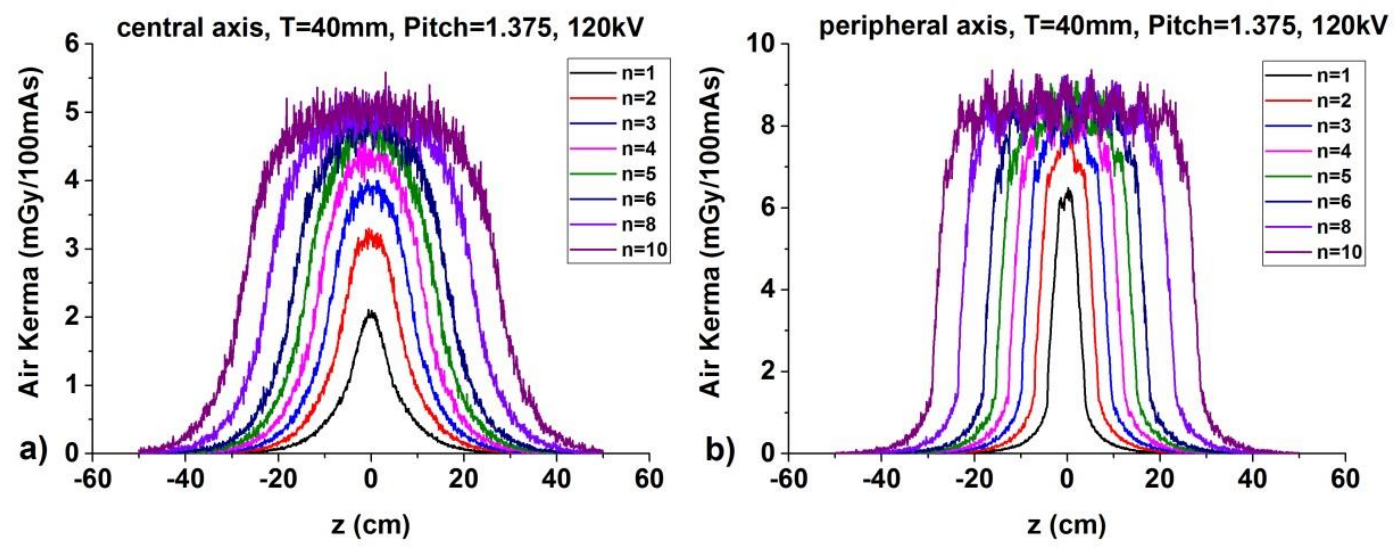

Figure 115. a) Central and b) peripheral cumulated dose profile in body CTDI phantom for helical scan with pitch=1.375.
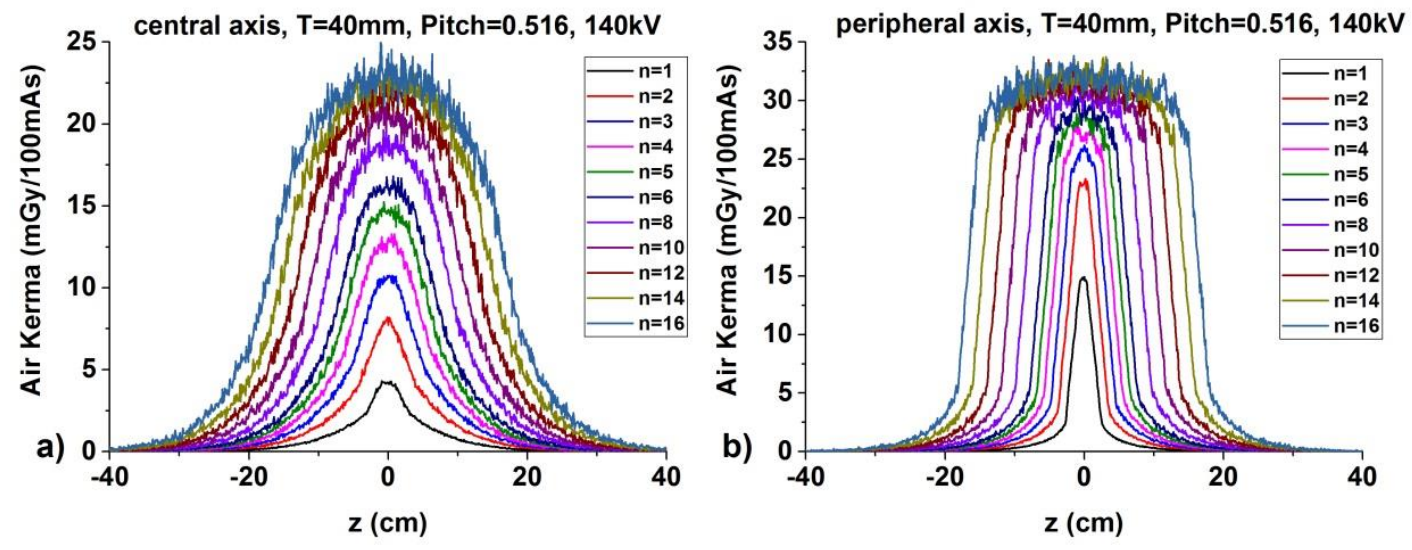

Figure 116. a) Central and b) peripheral cumulated dose profile in body CTDI phantom for helical scan with pitch=0.516. 

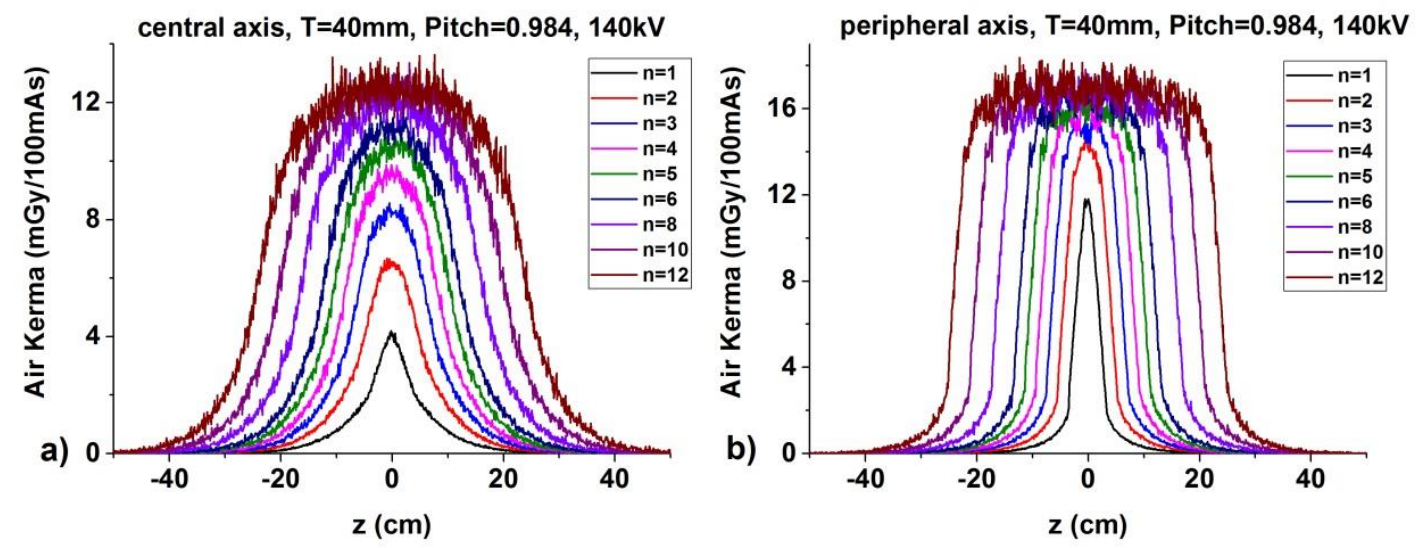

Figure 117. a) Central and b) peripheral cumulated dose profile in body CTDI phantom for helical scan with pitch=0.984.
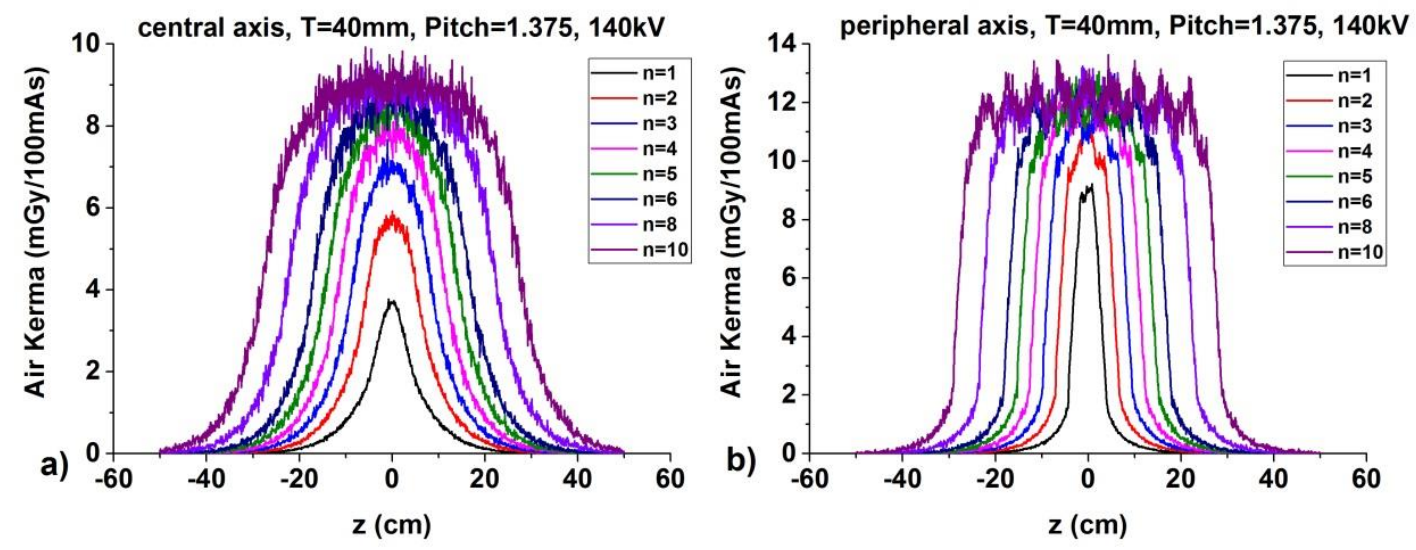

Figure 118. a) Central and b) peripheral cumulated dose profile in body CTDI phantom for helical scan with pitch=1.375. 
Appendix D: Dose Line Integral versus Scan Length.

- Head
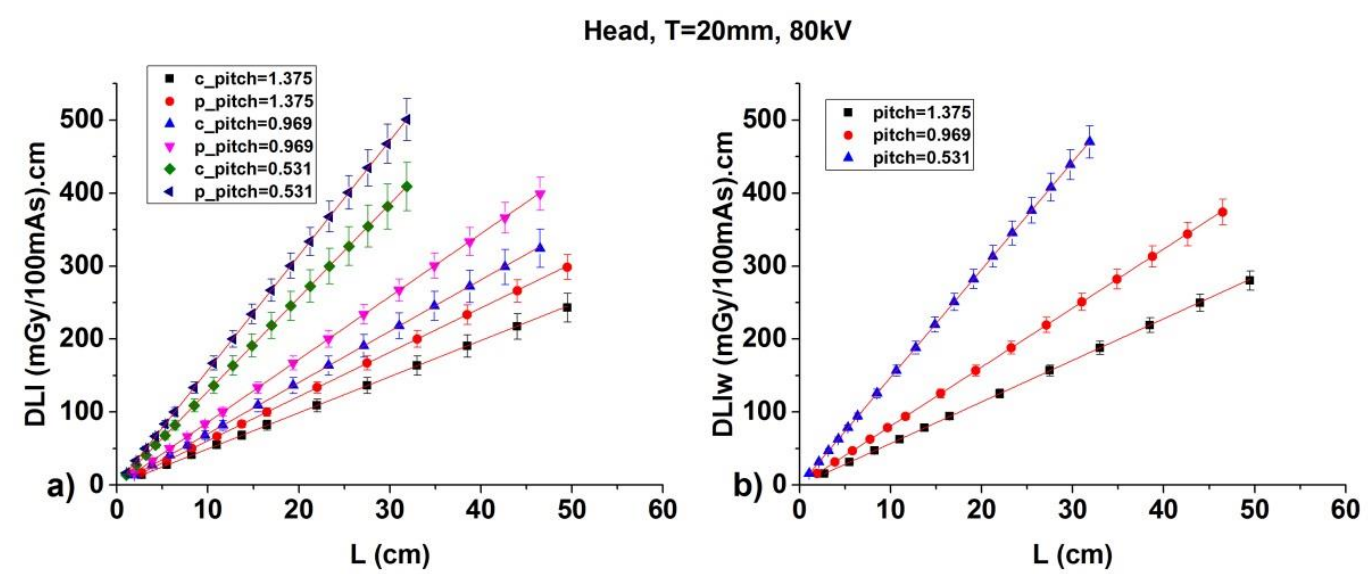

Figure 119. Simulated Dose Line Integral for head helical protocols of pitch values: $1.375,0.969$ and 0.531 at $80 \mathrm{kV}$ as a function of the scan length, $\mathrm{L}$, a) along the central (DLIc) and peripheral (DLIc) axes and for b) Weighted DLI, DLIw. Simulations were performed in an infinite cylindrical phantom of PMMA with $16 \mathrm{~cm}$ diameter and beam width of $20 \mathrm{~mm}$.
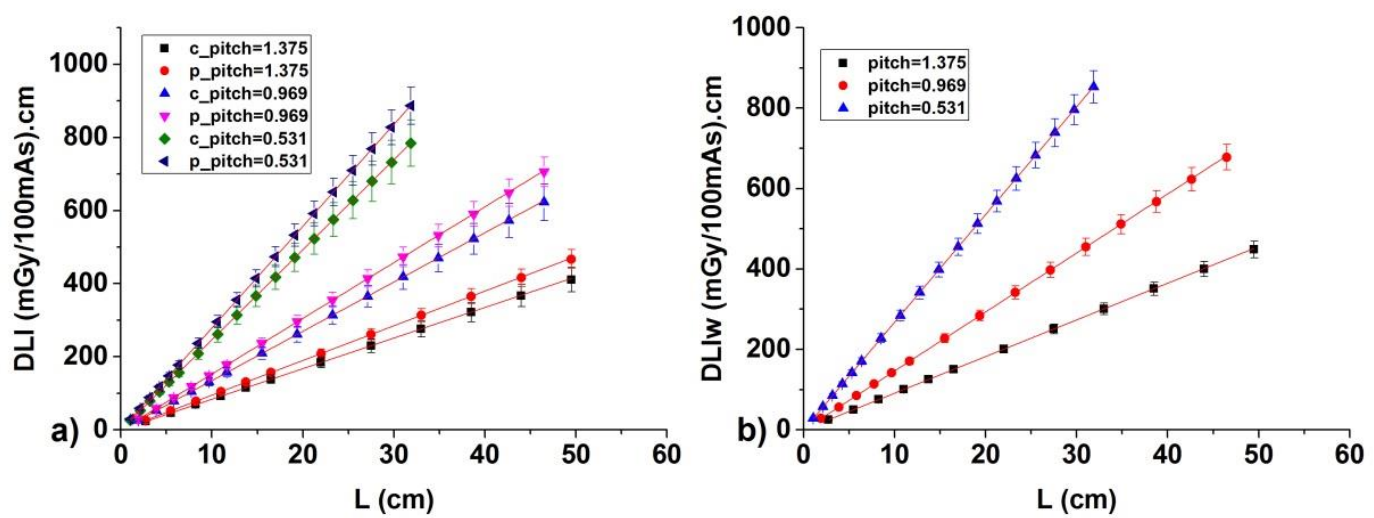

Figure 120. Simulated Dose Line Integral for head helical protocols of pitch values: $1.375,0.969$ and 0.531 at $100 \mathrm{kV}$ as a function of the scan length, $L$, a) along the central (DLIc) and peripheral (DLIc) axes and for b) Weighted DLI, DLIw. Simulations were performed in an infinite cylindrical phantom of PMMA with $16 \mathrm{~cm}$ diameter and beam width of $20 \mathrm{~mm}$ 


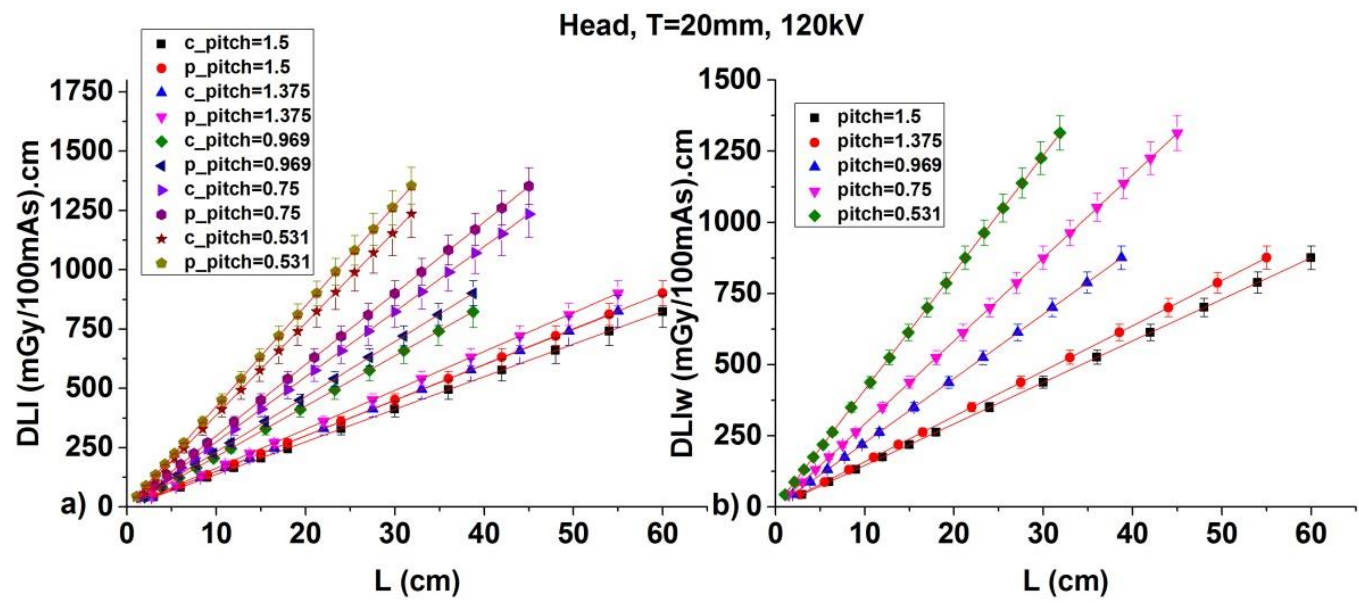

Figure 121. Simulated Dose Line Integral for head helical protocols of pitch values: $1.5,1.375,0.969,0.75$ and 0.531 at $120 \mathrm{kV}$ as a function of the scan length, $L$, a) along the central (DLIc) and peripheral (DLIc) axes and for b) Weighted DLI, DLIw. Simulations were performed in an infinite cylindrical phantom of PMMA with $16 \mathrm{~cm}$ diameter and beam width of $20 \mathrm{~mm}$.

Head, $\mathrm{T}=20 \mathrm{~mm}, 140 \mathrm{kV}$
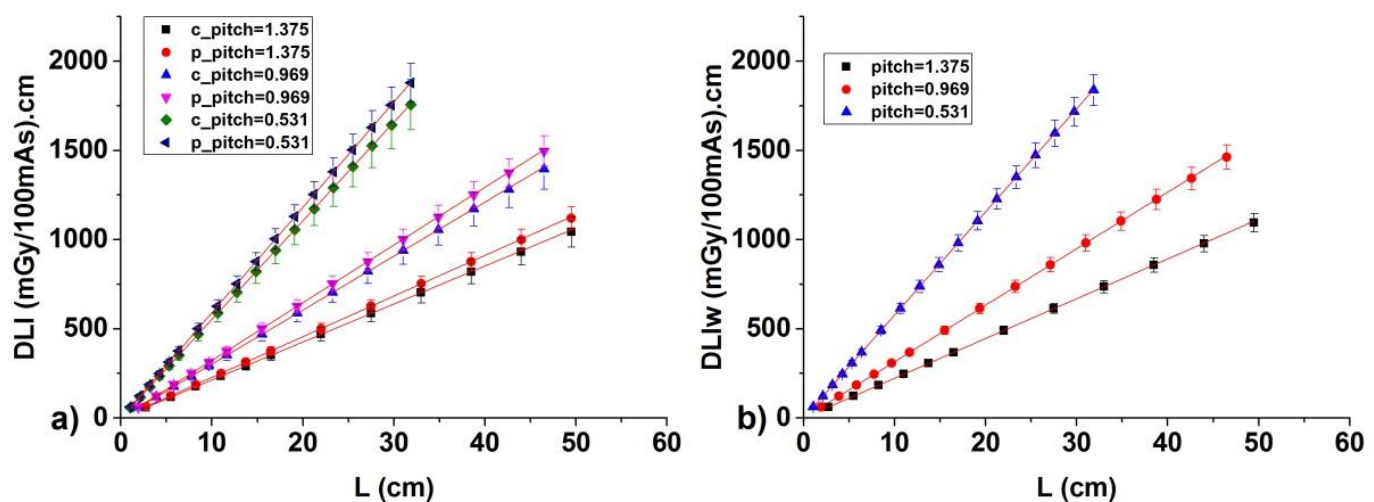

Figure 122. Simulated Dose Line Integral for head helical protocols of pitch values: $1.375,0.969$ and 0.531 at $140 \mathrm{kV}$ as a function of the scan length, $\mathrm{L}$, a) along the central (DLIc) and peripheral (DLIc) axes and for b) Weighted DLI, DLIw. Simulations were performed in an infinite cylindrical phantom of PMMA with $16 \mathrm{~cm}$ diameter and beam width of $20 \mathrm{~mm}$. 
- Body
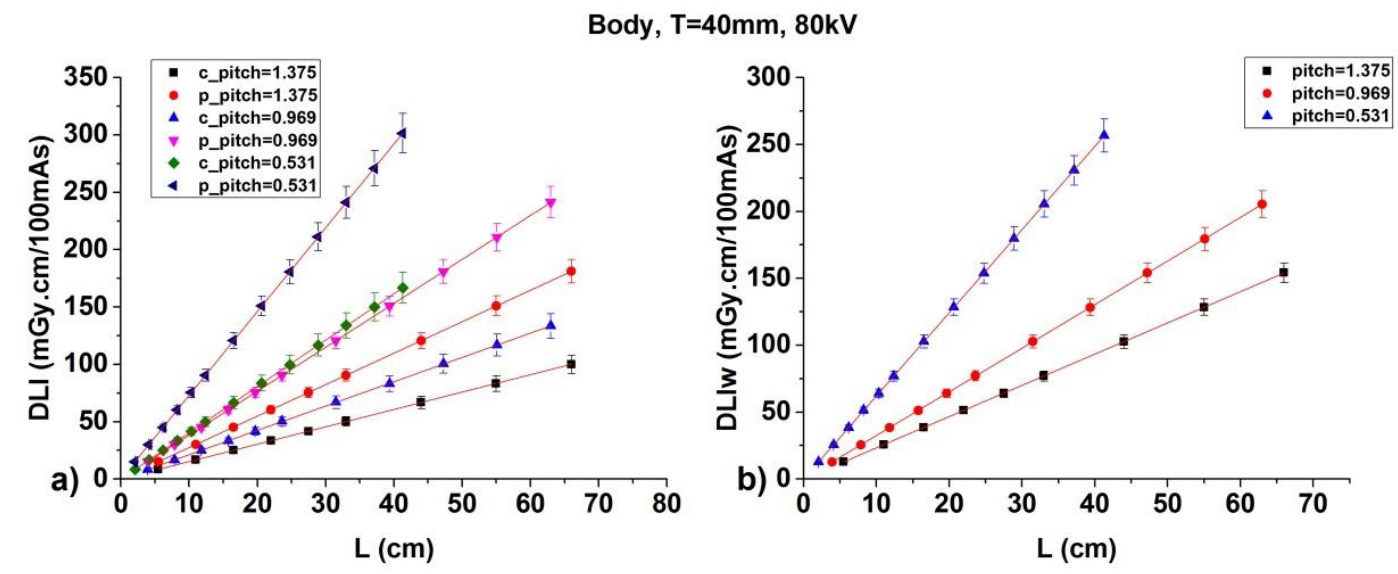

Figure 123. Simulated Dose Line Integral for body helical protocols of pitch values: 1.375, 0.984, and 0.516 at $80 \mathrm{kV}$ as a function of the irradiation length, L. Simulations were performed in an infinite cylindrical phantom of PMMA with $32 \mathrm{~cm}$ diameter and beam width of $40 \mathrm{~mm}$.
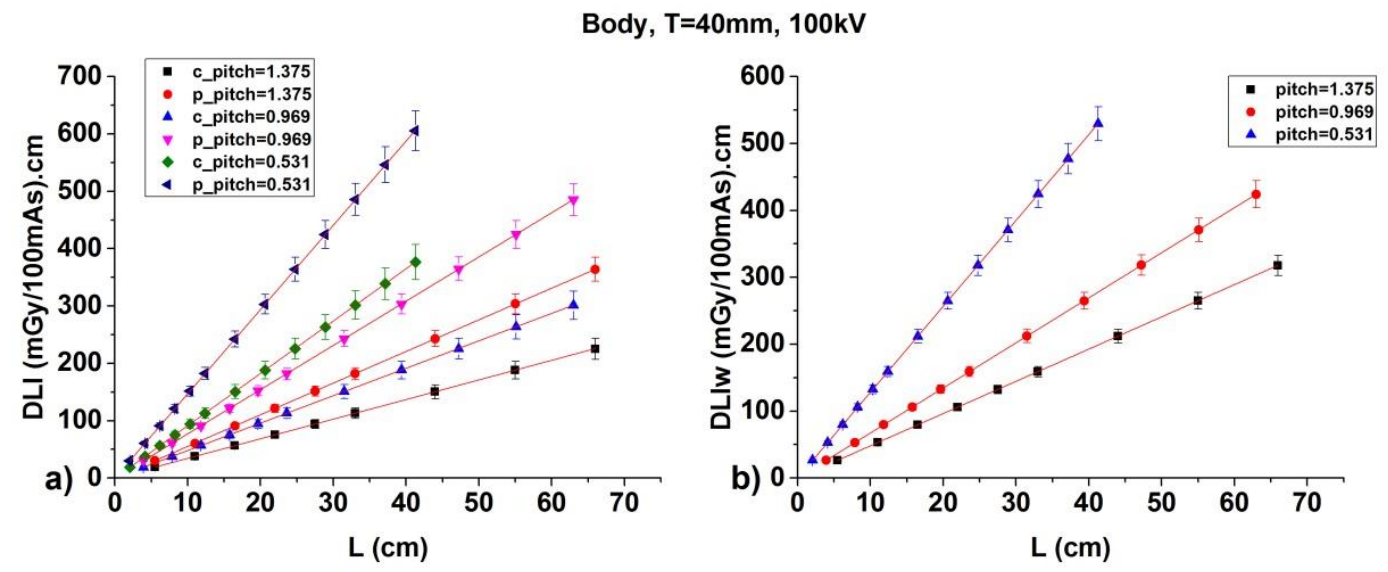

Figure 124. Simulated Dose Line Integral for body helical protocols of pitch values: $1.375,0.984$, and 0.516 at $100 \mathrm{kV}$ as a function of the irradiation length, L. Simulations were performed in an infinite cylindrical phantom of PMMA with $32 \mathrm{~cm}$ diameter and beam width of $40 \mathrm{~mm}$. 

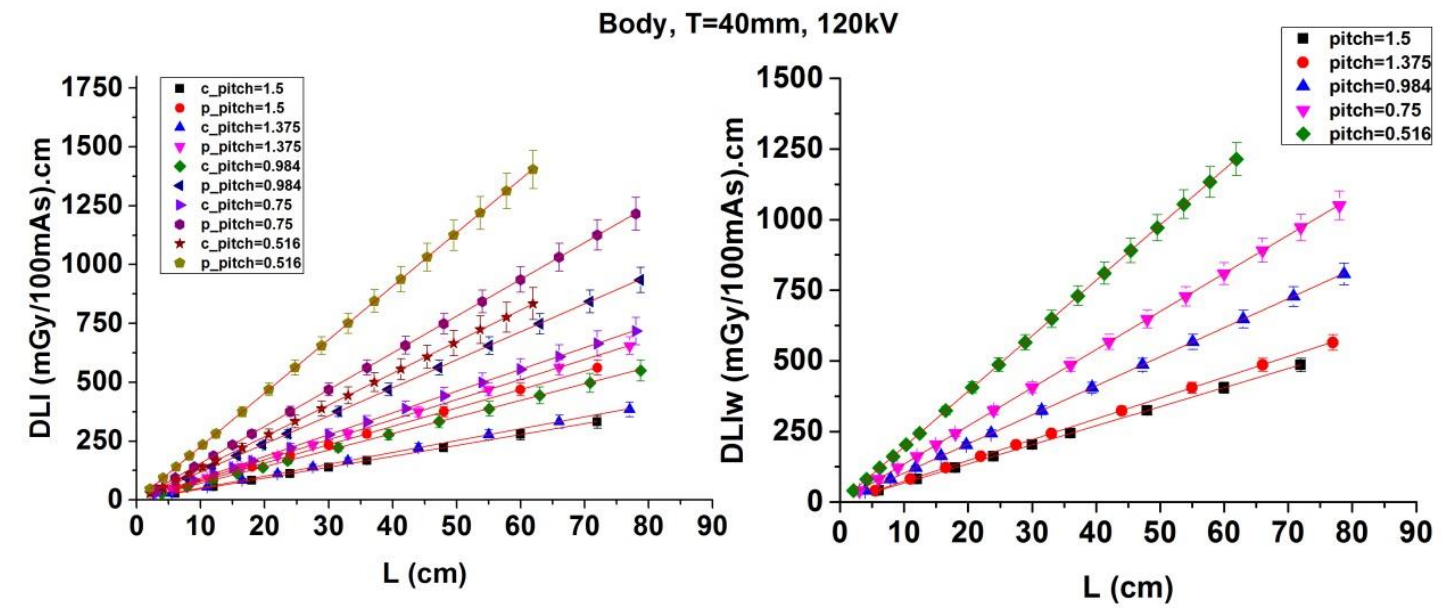

Figure 125 Simulated Dose Line Integral for body helical protocols of pitch values: 1.5, 1.375, 0.984, 0.75 and 0.516 at $120 \mathrm{kV}$ as a function of the irradiation length, $L$. Simulations were performed in an infinite cylindrical phantom of PMMA with $32 \mathrm{~cm}$ diameter and beam width of $40 \mathrm{~mm}$.

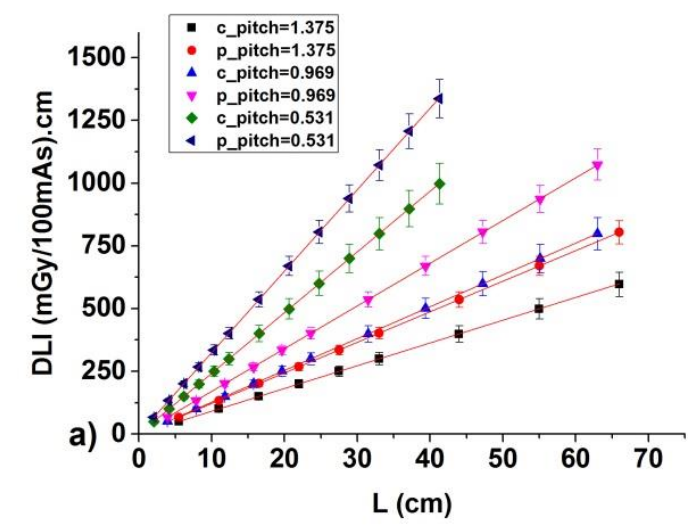

Body, $\mathrm{T}=40 \mathrm{~mm}, 140 \mathrm{kV}$

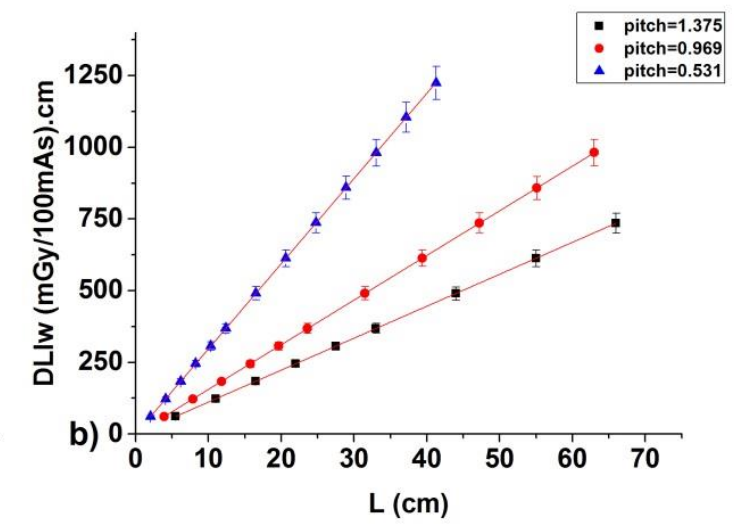

Figure 126. Simulated Dose Line Integral for body helical protocols of pitch values: $1.375,0.984$, and 0.516 at $140 \mathrm{kV}$ as a function of the irradiation length, $\mathrm{L}$. Simulations were performed in an infinite cylindrical phantom of PMMA with $32 \mathrm{~cm}$ diameter and beam width of $40 \mathrm{~mm}$. 
Appendix E: Rise to Equilibrium functions, assessing the Equilibrium Scanning Length, $L_{e q}$.

- Head
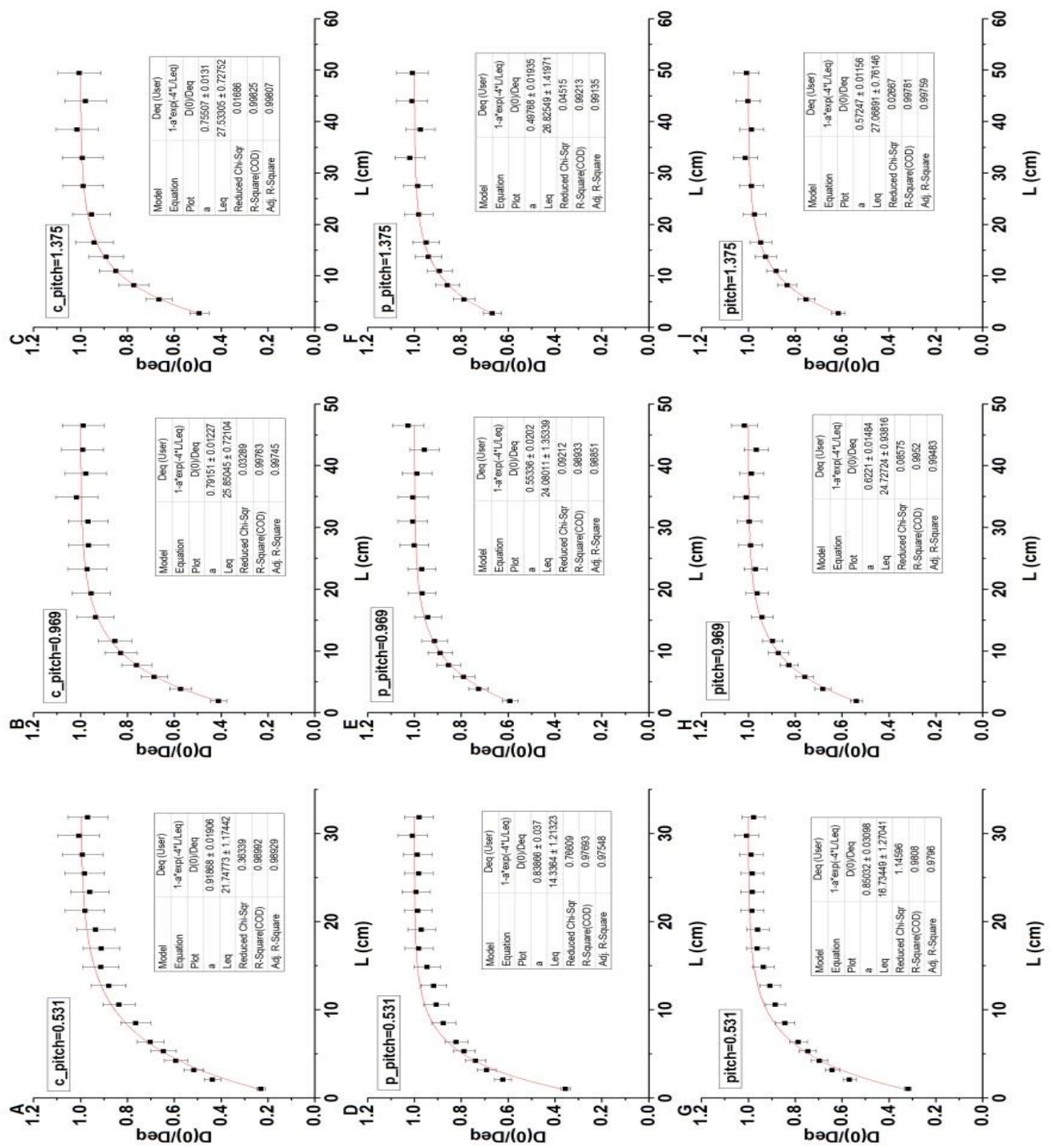

Figure 127. Equilibrium Length for $80 \mathrm{kV}$ head protocol 

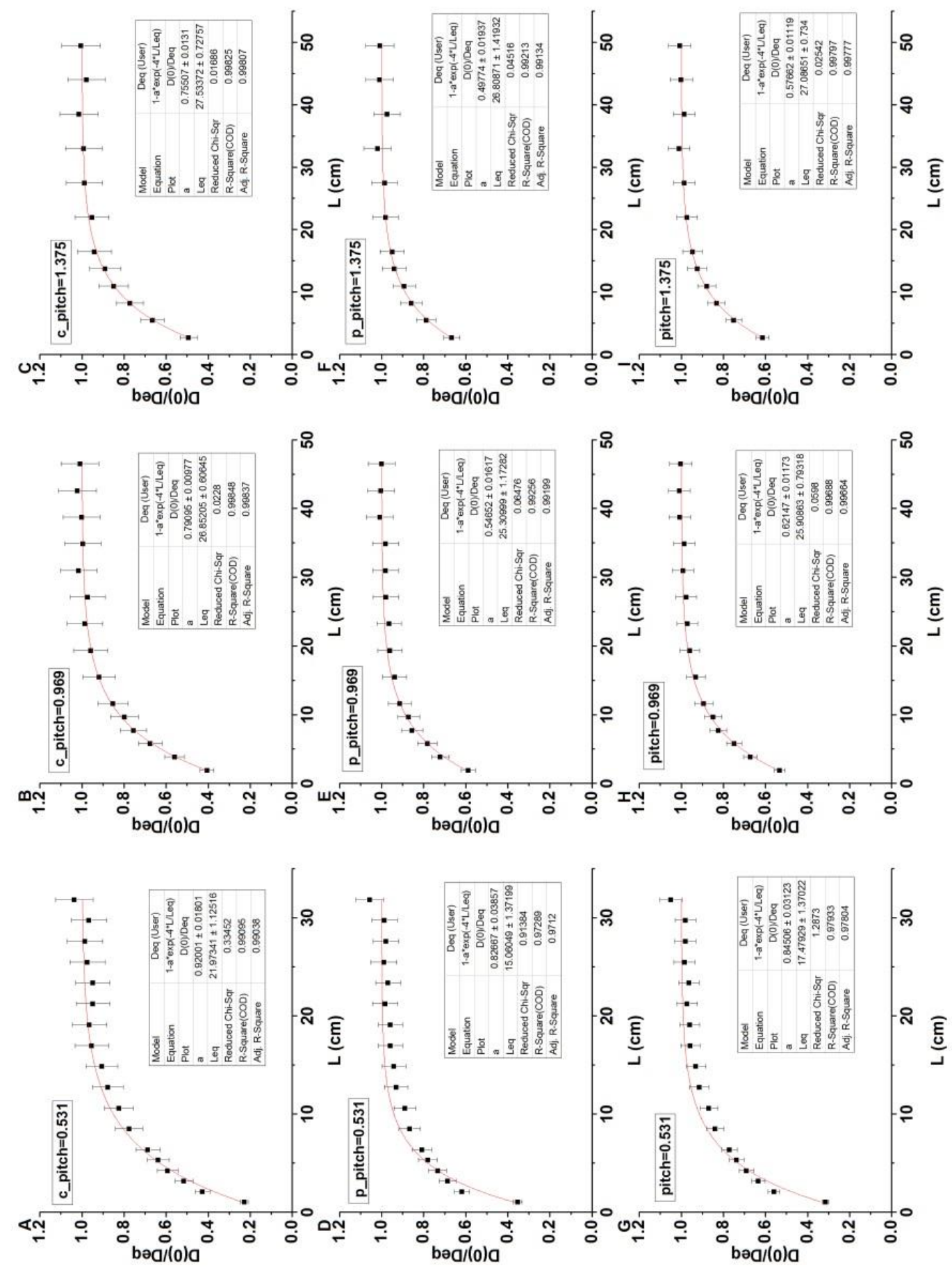

Figure 128. Equilibrium Length for $100 \mathrm{kV}$ head protocol 

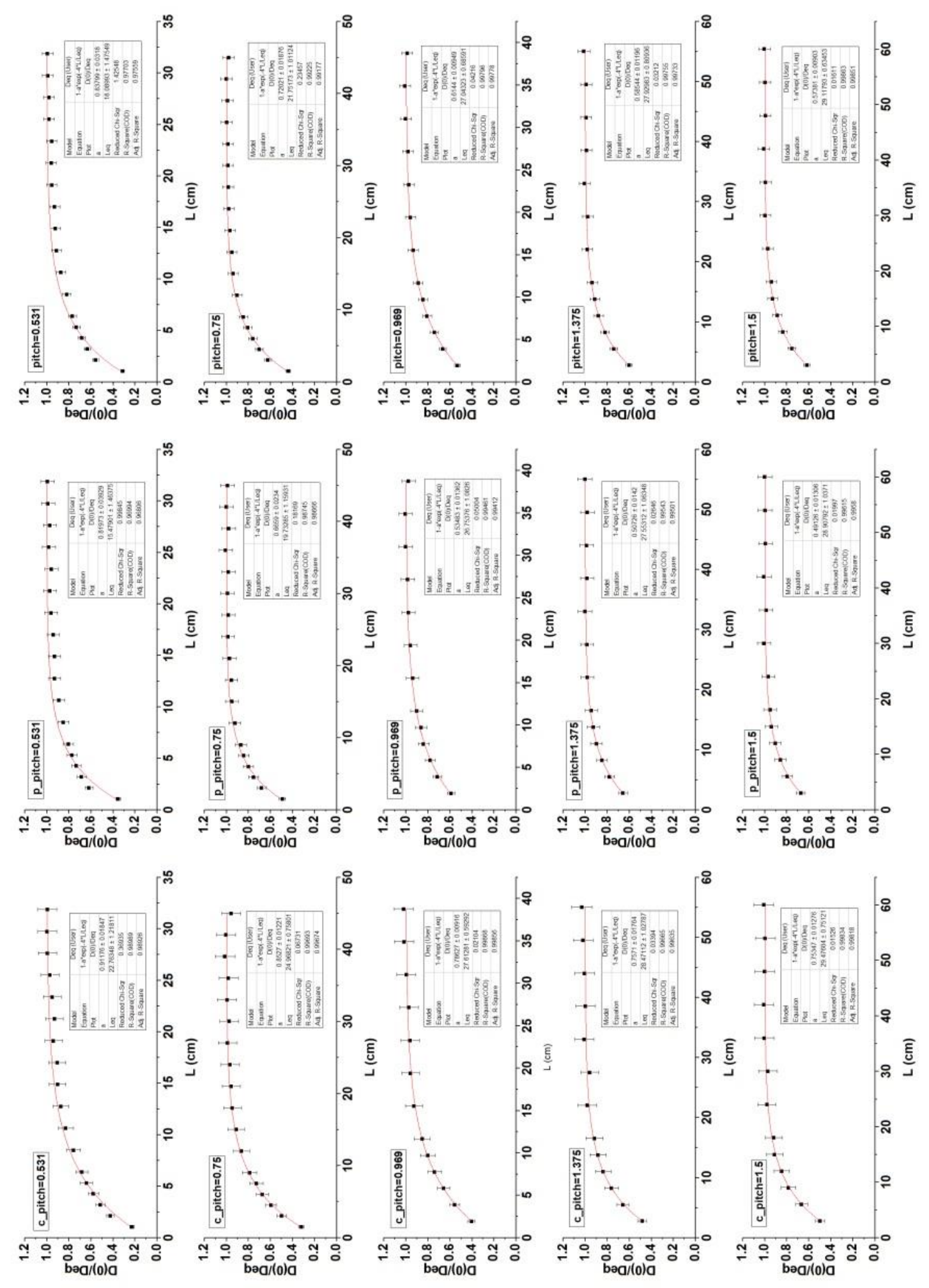

Figure 129. Equilibrium Length for $120 \mathrm{kV}$ head protocol 

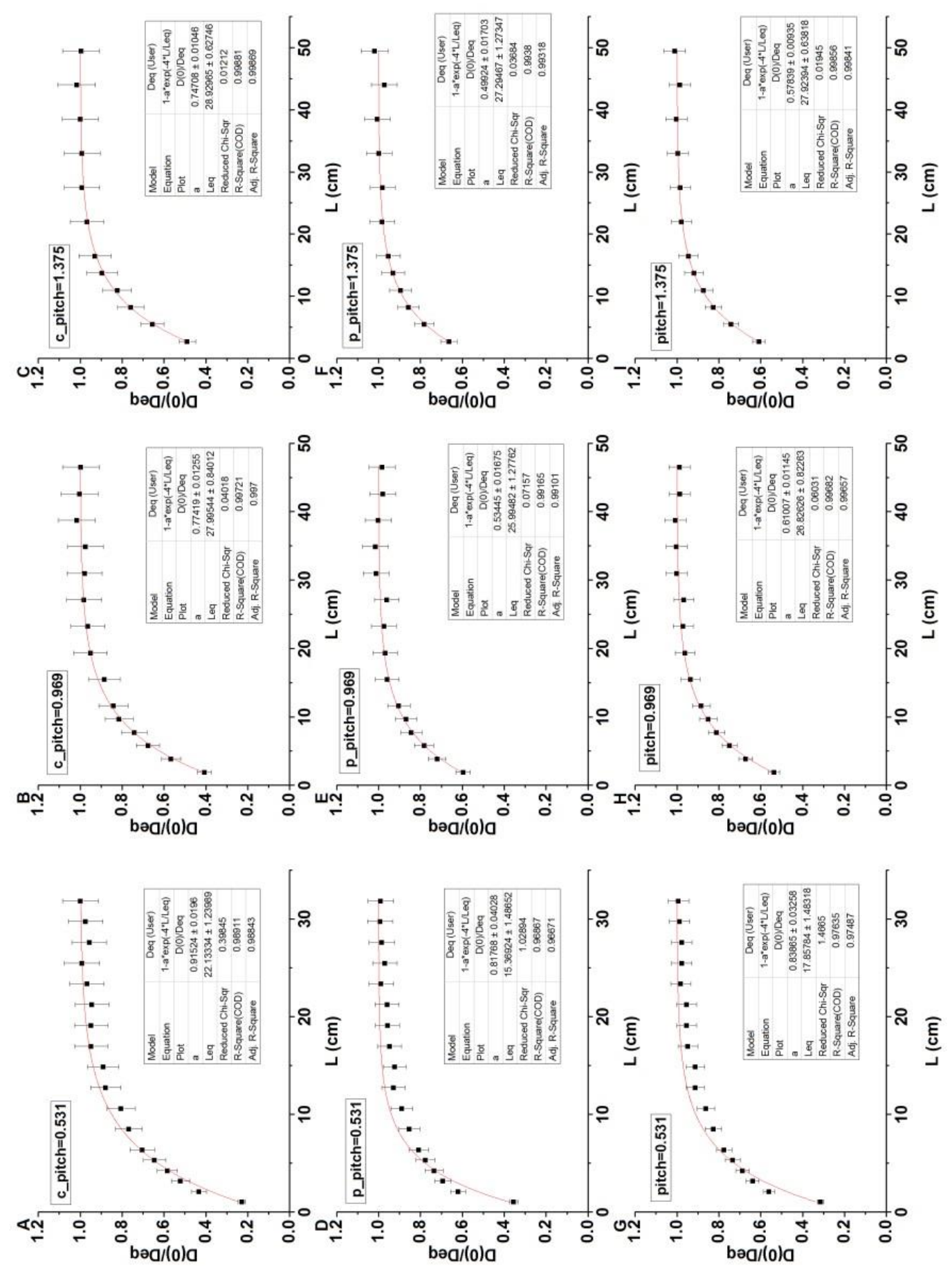

Figure 130. Equilibrium Length for $140 \mathrm{kV}$ head protocol 
- Body
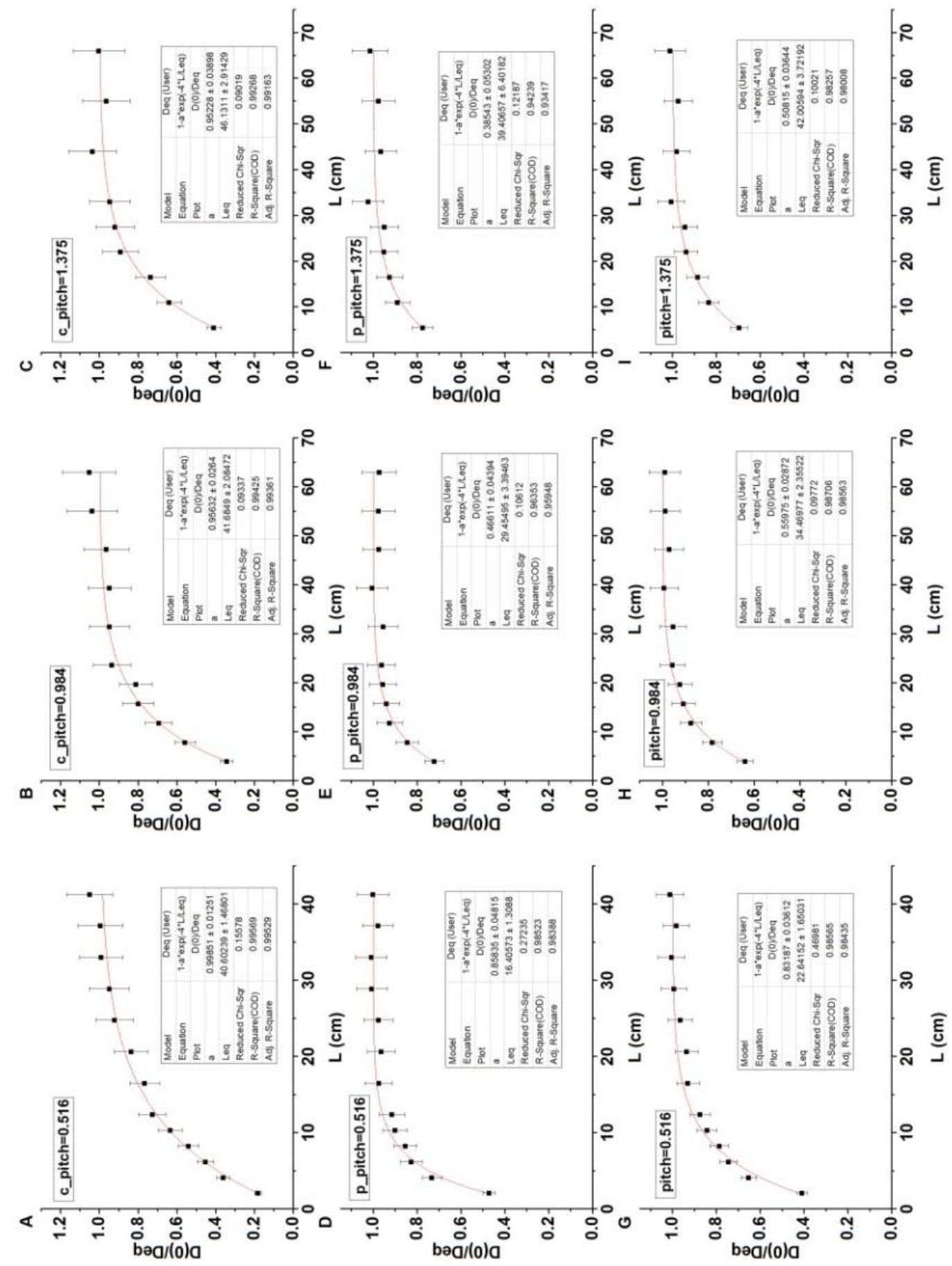

Figure 131. Equilibrium Length for $80 \mathrm{kV}$ body protocol 

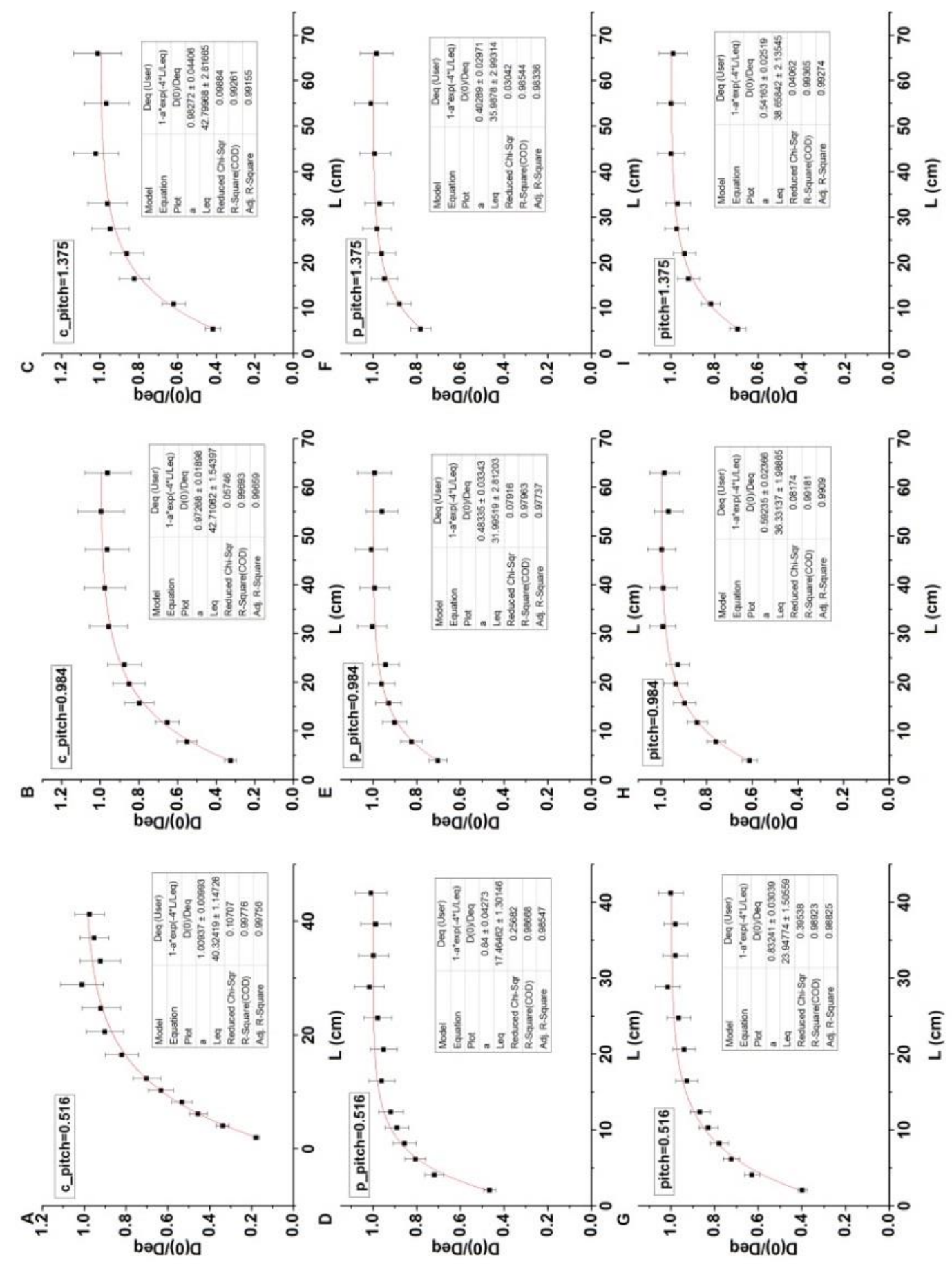

Figure 132. Equilibrium Length for $100 \mathrm{kV}$ body protocol 


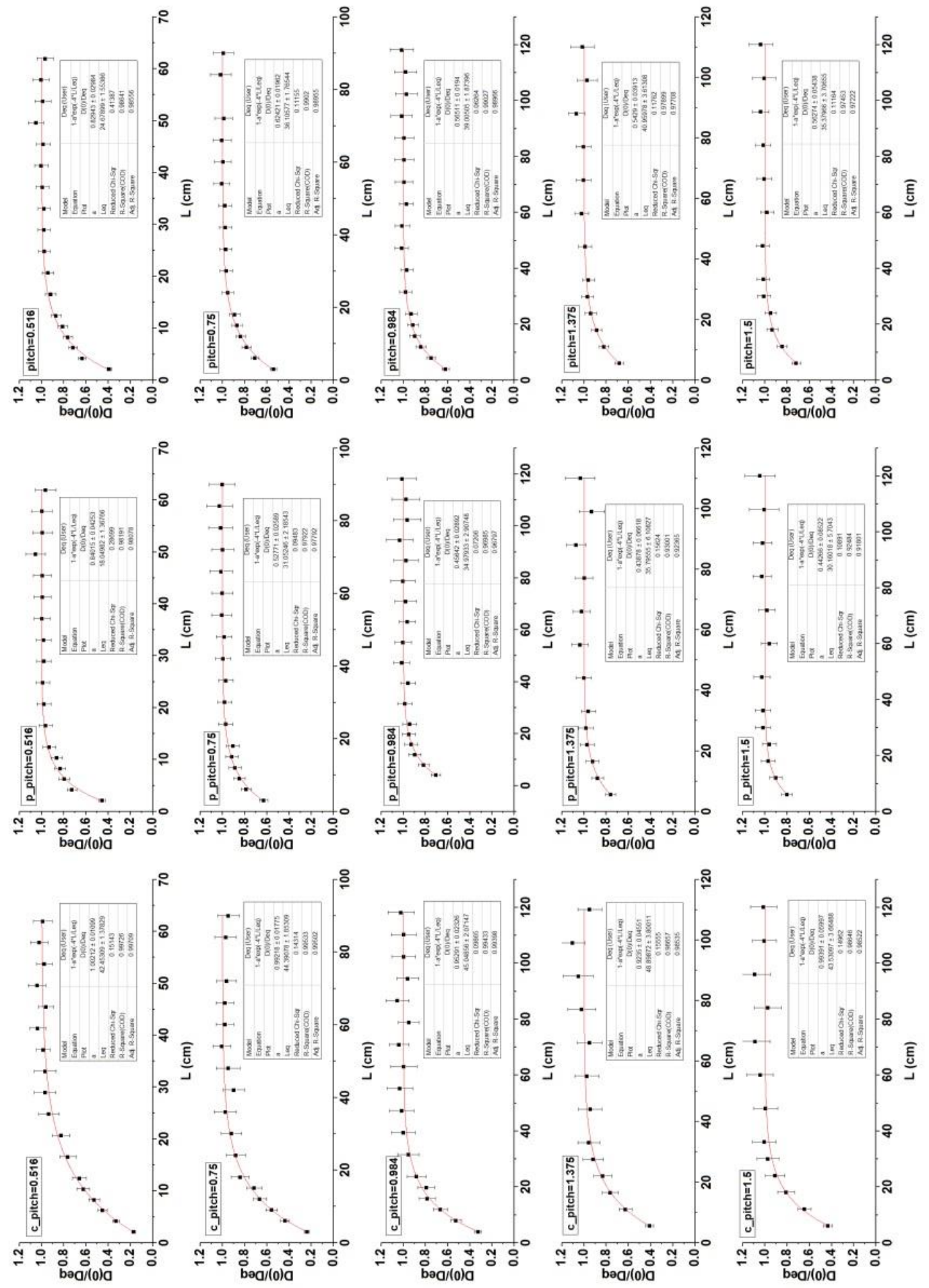

Figure 133. Equilibrium Length for $120 \mathrm{kV}$ body protocol 

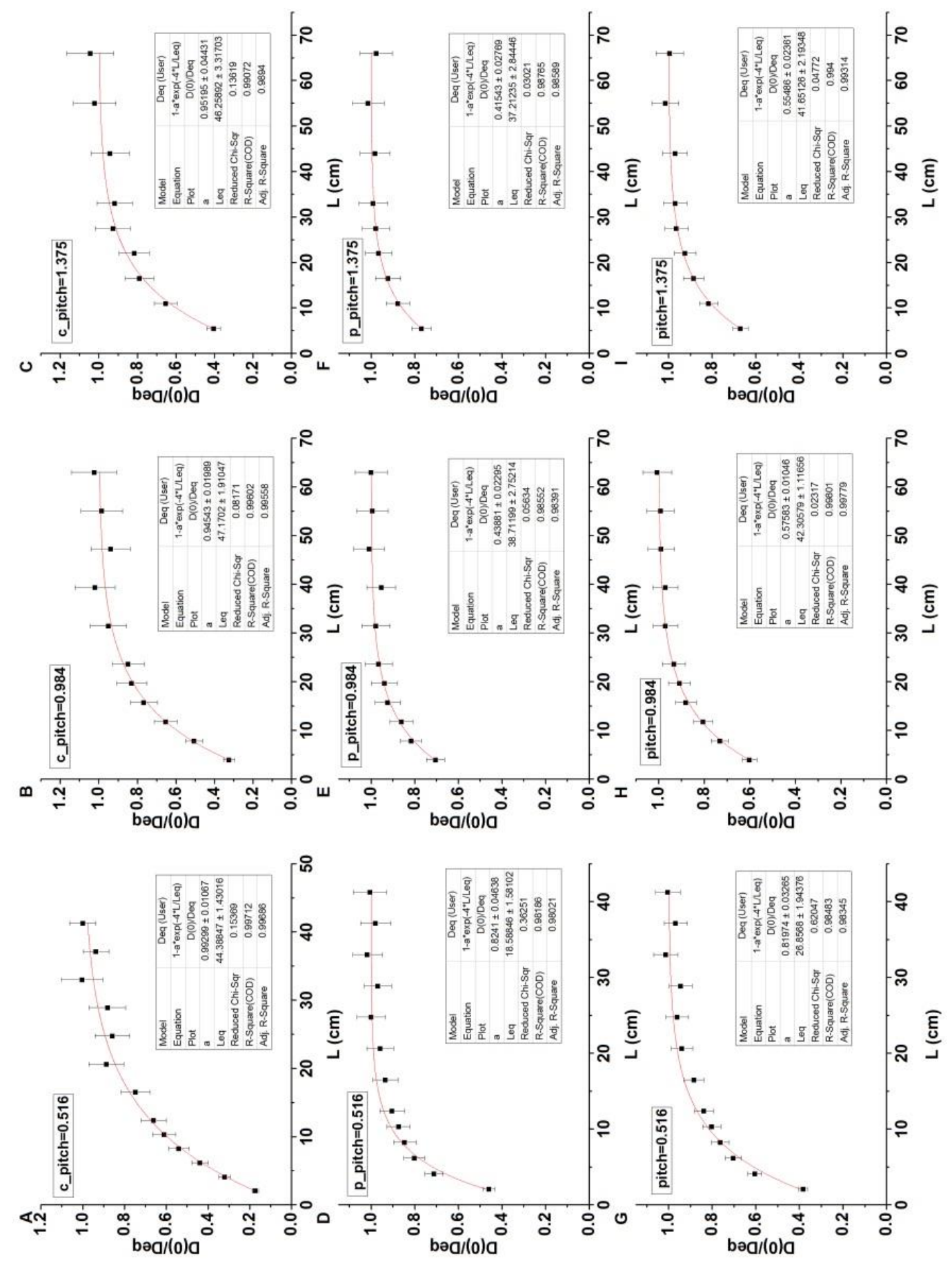

Figure 134. Equilibrium Length for $140 \mathrm{kV}$ body protocol 


\section{List of publications, conferences papers, posters and training sessions}

\section{- Publications during the Doctoral Studies period}

Alejandro H.L. Gonzalez, Josilene C. Santos; Paulo R. Costa, "Determinação dos Coeficientes de Conversão Médios entre Kerma no Ar e H*(10) usando Feixes de Raios X Primários, Secundários e Transmitidos na Faixa de Energia da Radiologia Diagnóstica", Revista Brasileira de Física Médica, (2017).

Josilene C. Santos; Alejandro H.L. Gonzalez; Ricardo A. Terini; Paulo R. Costa, "Determinação da camada semirredutora e da tensão aplicada a partir de espectros emitidos por um tubo de raios X", Revista Brasileira de Física Médica, (2017)

\section{- Conferences papers}

A H Lopez Gonzales, L P Robayo Puerto, P R Costa, "Computed tomography phase-space source model using the PENELOPE/PenEasy Monte Carlo code: implementation and validation", Proceeding of the International Conference on Applications of Radiation Science and Technology, (2017).

Abstract ID: 91 Image acquisition and material differentiation for. Dual Energy Computed Tomography by Monte Carlo simulations. Lorena Robayo, Alejandro H. Lopez, Paulo R. Costa. International Conference on Monte Carlo Techniques for Medical Applications (MCMA2017)

Abstract ID: 167 Assessing the $C T D I_{v o l}$ and SSDE from dose pro-files in cylindrical phantoms of water equivalent materials. Alejandro Lopez *, Lorena Robayo, Paulo Costa. International Conference on Monte Carlo Techniques for Medical Applications (MCMA2017)

\section{- Posters}

Alejandro H. Lopez, Paulo R. Costa. Influence of the tube current modulation on CT cumulative dose profile for central and peripheral cylindrical phantom axis. CÓDIGO DE TRABAJO: PA193. Asociacion Latinoamericana de Fisica Medica (ALFIM). 
Lopez Gonzales, A.H., Robayo Puerto, L. P. Costa, P.R.. Assessing the CTDI vol $_{\text {and SSDE }}$ from dose profiles in cylindrical phantoms of water equivalent materials. International Conference on Monte Carlo Techniques for Medical Applications (MCMA2017).

A.H. Lopez Gonzales, L.P. Robayo Puerto, P.R. Costa. Computed tomography phase-space source model using the PENELOPE/PenEasy Monte Carlo code: implementation and validation. International Conference on Applications of Radiation Science and Technology (ICARST 2017)24 to 28 April 2017, Vienna, Austria. ID: PB-09. 


\section{References}

AAPM-EFOMP 2019. Report No. 246 - Estimating Patient Organ Dose with Computed Tomography: A Review of Present Methodology and Required DICOM Information. One Physics Ellipse, College Park, MD 20740-3846.

AAPM 1977. Phantoms for performance evaluation and quality assurance of CT scanners. Diagnostic Radiology Committe Task Force on CT Scanner Phantoms.

AAPM. 2008. Report No. 096 - The Measurement, Reporting, and Management of Radiation Dose in CT [Online]. One Physics Ellipse, College Park, MD 20740-3846: American Association of Physicists in Medicine.

AAPM 2010. Report No. 111 - Comprehensive Methodology for the Evaluation of Radiation Dose in X-Ray Computed Tomography. One Physics Ellipse, College Park, MD 20740-3846.

AAPM 2011. Size-Specific Dose Estimates (SSDE) in Pediatric and Adult Body CT Examinations. Report of AAPM Task Group 204, The American Association of Physicists in Medicine.

AAPM 2014a. Report No. 220 - Use of Water Equivalent Diameter for Calculating Patient Size and Size-Specific Dose Estimates (SSDE) in CT. One Physics Ellipse, College Park, MD 20740-3846.

AAPM 2014b. Use of Water Equivalent Diameter for Calculating Patient Size and SizeSpecific Dose Estimates (SSDE) in CT. Report of AAPM Task Group 220, The American Association of Physicists in Medicine.

ABEL, N. H. 1826. Auflösung einer mechanischen Aufgabe. Journal für die reine und angewandte Mathematik, 1, 5.

ACHENBACH, S. \& KONDO, T. 2012. Technical Advances in Cardiac CT. Cardiology Clinics, 30, 1-8.

ALEXANDER, A., MCGILL, M., TARASOVA, A., FERREIRA, C. \& ZURKIYA, D. 2019. Scanning the Future of Medical Imaging. Journal of the American College of Radiology, 16, 501-507.

ALMANSA, J., SALVAT-PUJOL, F., DÍAZ-LONDOÑO, G., CARNICER, A., LALLENA, A. M. \& SALVAT, F. 2016. PENGEOM-A general-purpose geometry package for Monte Carlo simulation of radiation transport in material systems defined by quadric surfaces. Computer Physics Communications, 199, 102-113.

AMBROSE, J. 1973. Computerized transverse axial scanning (tomography): Part 2. Clinical application. The British Journal of Radiology, 46, 1023-1047.

ANDREO, P. 1991. Monte Carlo techniques in medical radiation physics. Physics in Medicine and Biology, 36, 861-920.

ANDREO, P. 2018. Monte Carlo simulations in radiotherapy dosimetry. Radiation Oncology, $13,121$.

ANDREO, P. 2019. Data for the dosimetry of low- and medium-energy kV x rays. Physics in Medicine \& Biology, 64, 205019.

ATHERTON, J. V. \& HUDA, W. 1995. CT doses in cylindrical phantoms. Physics in Medicine and Biology, 40, 891.

ATTIX, F. H. 1986. Introduction to Radiological Physics and Radiation Dosimetry, Wiley.

BAERT, A. L. \& PASSARIELLO, R. 2010. Multidetector-Row CT Angiography, Springer Berlin Heidelberg. 
BEHLING, R. 2015. Modern Diagnostic X-Ray Sources: Technology, Manufacturing, Reliability, CRC Press.

BEHLING, R. \& GRÜNER, F. 2018. Diagnostic X-ray sources-present and future. Nuclear Instruments and Methods in Physics Research Section A: Accelerators, Spectrometers, Detectors and Associated Equipment, 878, 50-57.

BERGER, M. J. 1963. Monte Carlo calculation of the penetration and diffusion of fast charged particles, New York, Academic Press.

BERGER, M. J. \& SELTZER, S. M. 1972. Response functions for sodium iodide scintillation detectors. Nuclear Instruments and Methods, 104, 317-332.

BIELAJEW, A. F. \& ROGERS, D. W. O. 1986. Presta: The parameter reduced electron-step transport algorithm for electron monte carlo transport. Nuclear Instruments and Methods in Physics Research Section B: Beam Interactions with Materials and Atoms, 18, 165-181.

BMJ 1899. Special Correspondence. British medical journal, 1, 877-878.

BOONE, J. M. 2007. The trouble with CTDI100. Medical Physics, 34, 1364-1371.

BOONE, J. M. 2009. Dose spread functions in computed tomography: A Monte Carlo study. Medical Physics, 36, 4547-4554.

BRACEWELL, R. N. 2000. The Fourier Transform and its Applications, Boston, McGrawHill.

BRENNER, D. J., MCCOLLOUGH, C. H. \& ORTON, C. G. 2006. It is time to retire the computed tomography dose index (CTDI) for CT quality assurance and dose optimization. Medical Physics, 33, 1189-1191.

BRUAllA, L., SALVAT, F. \& PALANCO-ZAMORA, R. 2009. Efficient Monte Carlo simulation of multileaf collimators using geometry-related variance-reduction techniques. Physics in Medicine and Biology, 54, 4131.

BUSH, K., ZAVGORODNI, S. F. \& BECKHAM, W. A. 2007. Azimuthal particle redistribution for the reduction of latent phase-space variance in Monte Carlo simulations. Physics in Medicine and Biology, 52, 4345.

BUSHBERG, J. T. 2002. The Essential Physics of Medical Imaging, Lippincott Williams \& Wilkins.

BUSHBERG, J. T., SEIBERT, J. A., LEIDHOLDT, E. M. \& BOONE, J. M. 2011. The Essential Physics of Medical Imaging, Wolters Kluwer Health.

BUZUG, T. M. 2008. Computed Tomography: From Photon Statistics to Modern Cone-Beam $C T$, Springer Berlin Heidelberg.

CHETTY, I. J., CURRAN, B., CYGLER, J. E., DEMARCO, J. J., EZZELL, G., FADDEGON, B. A., KAWRAKOW, I., KEALL, P. J., LIU, H., MA, C.-M. C., ROGERS, D. W. O., SEUNTJENS, J., SHEIKH-BAGHERI, D. \& SIEBERS, J. V. 2007. Report of the AAPM Task Group No. 105: Issues associated with clinical implementation of Monte Carlo-based photon and electron external beam treatment planning. Medical Physics, 34, 4818-4853.

CHO, S. H., VASSILIEV, O. N., LEE, S., LIU, H. H., IBBOTT, G. S. \& MOHAN, R. 2005. Reference photon dosimetry data and reference phase space data for the $6 \mathrm{MV}$ photon beam from Varian Clinac 2100 series linear accelerators. Medical Physics, 32, 137148.

CIERNIAK, R. 2011. X-Ray Computed Tomography in Biomedical Engineering, SpringerVerlag London.

CORMACK, A. 1980. Early two-dimensional reconstruction and recent topics stemming from it. Science, 209, 1482-1486. 
CORMACK, A. M. 1963. Representation of a Function by Its Line Integrals, with Some Radiological Applications. Journal of Applied Physics, 34, 2722-2727.

CORMACK, A. M. 1973. Reconstruction of densities from their projections, with applications in radiological physics. Physics in Medicine and Biology, 18, 195-207.

CORMACK, A. M. \& M., C. A. 1964. Representation of a Function by Its Line Integrals, with Some Radiological Applications. II. Journal of Applied Physics, 35, 2908-2913.

COSTA, P. 1993. Eficiência Geométrica em Tomografia Computadorizada: Modelo Generalizado Instituto de Física, Universidade de São Paulo., Dissertação de Mestrado.

COSTA, P. R., NERSISSIAN, D. Y., SALVADOR, F. C., RIO, P. B. \& CALDAS, L. V. E. 2007. Generation of calibrated tungsten target X-ray spectra: Modified TBC model. Health Phys, 92, 24-32.

CROCKER, H. R. 1897. A Case of Dermatitis from Roentgen Rays. British medical journal, $1,8-9$.

DIXON, R. 2019. The Physics of CT Dosimetry: Ctdi and Beyond, CRC Press, Taylor \& Francis Group.

DIXON, R. L. 2003. A new look at CT dose measurement: Beyond CTDI. Medical Physics, $30,1272-1280$.

DIXON, R. L. \& BALLARD, A. C. 2007. Experimental validation of a versatile system of CT dosimetry using a conventional ion chamber: Beyond. Medical Physics, 34, 33993413.

DIXON, R. L. \& BOONE, J. M. 2010. Cone beam CT dosimetry: A unified and selfconsistent approach including all scan modalities - With or without phantom motion. Medical Physics, 37, 2703-2718.

DIXON, R. L. \& BOONE, J. M. 2011. Analytical equations for CT dose profiles derived using a scatter kernel of Monte Carlo parentage with broad applicability to CT dosimetry problems. Med Phys, 38, 4251-64.

DIXON, R. L. \& BOONE, J. M. 2013. Dose equations for tube current modulation in CT scanning and the interpretation of the associated CTDIvol. Medical Physics, 40, 111920 .

DIXON, R. L. \& BOONE, J. M. 2014. Stationary table CT dosimetry and anomalous scannerreported values of CTDIvol. Medical Physics, 41, 011907.

DIXON, R. L. \& EKSTRAND, K. E. 1978. A Film Dosimetry System for Use in Computed Tomography. Radiology, 127, 255-258.

DIXON, R. L., MUNLEY, M. T. \& BAYRAM, E. 2005. An improved analytical model for CT dose simulation with a new look at the theory of CT dose. Medical Physics, 32, 3712-3728.

EC 1998. European Commission's Study Group: Quality criteria for computed tomography.

FDA, U. S. 1984. Code of Federal Regulations, Diagnostic x-ray systems and their major components 21 CFR 1020.33 Govt. Printing Office.

FERAS, M. O. A.-D., ROJAS, E. L. \& ANTONIO, M. L. 2005. Effects of bone- and airtissue inhomogeneities on the dose distributions of the Leksell Gamma Knife® calculated with PENELOPE. Physics in Medicine and Biology, 50, 5665.

FERNÁNDEZ-VAREA, J. M., MAYOL, R., BARÓ, J. \& SALVAT, F. 1993. On the theory and simulation of multiple elastic scattering of electrons. Nuclear Instruments and Methods in Physics Research Section B: Beam Interactions with Materials and Atoms, 73, 447-473.

GAGNE, R. M. 1989. Geometrical aspects of computed tomography: Sensitivity profile and exposure profile. Medical Physics, 16, 29-37. 
GE. 2019. Time line of CT [Online]. Available: http://newsroom.gehealthcare.com/radiological-diagnosis-40-years-on-the-evolutionof-ct-imaging/ [Accessed 12/13/2019.

GIANSANTE, L., SANTOS, J. C., UMISEDO, N. K., TERINI, R. A. \& COSTA, P. R. 2018. Characterization of OSL dosimeters for use in dose assessment in Computed Tomography procedures. Physica Medica, 47, 16-22.

HAYWARD, E. \& HUBBELL, J. 1954. The Albedo of Various Materials for 1-Mev Photons. Physical Review, 93, 955-956.

HOSNY, A., PARMAR, C., QUACKENBUSH, J., SCHWARTZ, L. H. \& AERTS, H. J. W. L. 2018. Artificial intelligence in radiology. Nature reviews. Cancer, 18, 500-510.

HOUNSFIELD, G. N. 1973. Computerized transverse axial scanning (tomography): Part 1. Description of system. The British Journal of Radiology, 46, 1016-1022.

HSIEH, J. 2009. Computed Tomography Principles, Design, Artifacts, and Recent Advances, Wiley.

HUDA, W. 2010. Review of Radiologic Physics, Lippincott Williams \& Wilkins.

IAEA 2011. Implementation of the International Code of Practice on Dosimetry in Diagnostic Radiology (TRS 457): Review of Test Results. Viena.

ICRU 2012. Report No. 87: Radiation dose and image-quality assessment in computed tomography. Journal of the International Commission on Radiation Units and Measurements.

IEC 2001. Medical Electrical Equipment: Part 2-44: Particular requirements for the safety of $\mathrm{X}$-ray equipment for computed tomography (Amendment 1 ).

IEC 2004. Evaluation and routine testing in medical imaging departments - Part 3-5: Acceptance tests - Imaging performance of computed tomography X-ray equipment.

IEC 2005. Medical Diagnostic X-ray Equipment-Radiation Conditions for Use in the Determination of Characteristics. IEC 61267 (Geneva: International Electrotechnical Commission).

JACKSON, J. D. 1975. Classical electrodynamics, Wiley.

JENSEN, K., MARTINSEN, A. C. T., TINGBERG, A., AALØKKEN, T. M. \& FOSSE, E. 2014. Comparing five different iterative reconstruction algorithms for computed tomography in an ROC study. European Radiology, 24, 2989-3002.

JERAJ, R., MACKIE, T. R., BALOG, J., OLIVERA, G., PEARSON, D., KAPATOES, J., RUCHALA, K. \& RECKWERDT, P. 2004. Radiation characteristics of helical tomotherapy. Medical Physics, 31, 396-404.

JOCELYNE, M., JEAN, G., BRUNO, C. \& JEAN, B. 2001. Calculation of perturbation correction factors for some reference dosimeters in high-energy photon beams with the Monte Carlo code PENELOPE. Physics in Medicine and Biology, 46, 1707.

JUCIUS, R. A. \& KAMBIC, G. X. 1977. Radiation Dosimetry In Computed Tomography (CT). Application of Optical Instrumentation in Medicine VI. SPIE.

KAHN, H. 1950a. Random sampling (Monte Carlo) techniques in neutron attenuation problems--I. Nucleonics, 6, 5.

KAHN, H. 1950b. Random sampling (Monte Carlo) techniques in neutron attenuation problems--II. Nucleonics, 6, 6.

KALENDER, W., SEISSLER, W. \& VOCK, P. 1989. Single-breath-hold spiral volumetric CT by continuous patient translation and scanner rotation, United States, Radiological Society of North America Inc.

KALENDER, W. A. 2011. Computed Tomography: Fundamentals, System Technology, Image Quality, Applications, 3rd Edition, Publicis. 
KAMBIC, G. X. \& JUCIUS, R. A. 1979. Pencil shaped ionization chamber for exposure from a computed tomography scan. Medical Physics, 6, 459-459.

KRAMER, R., CASSOLA, V. F., ANDRADE, M. E. A., ARAÚJO, M. W. C. D., BRENNER, D. J. \& KHOURY, H. J. 2017. Mathematical modelling of scannerspecific bowtie filters for Monte Carlo CT dosimetry. Physics in Medicine and Biology, 62, 781.

KRAMERS, H. A. 1923. XCIII. On the theory of X-ray absorption and of the continuous Xray spectrum. The London, Edinburgh, and Dublin Philosophical Magazine and Journal of Science, 46, 836-871.

KUHN, T. S. \& HACKING, I. 2012. The Structure of Scientific Revolutions: 50th Anniversary Edition, University of Chicago Press.

LEITZ, W., AXELSSON, B. \& SZENDRO, G. 1995. Computed Tomography Dose Assessment - A Practical Approach. Radiation Protection Dosimetry, 57, 377-380.

LELL, M. M. \& KACHELRIEß, M. 2019. Recent and Upcoming Technological Developments in Computed Tomography: High Speed, Low Dose, Deep Learning, Multienergy. Investigative Radiology, Publish Ahead of Print.

LELL, M. M., WILDBERGER, J. E., ALKADHI, H., DAMILAKIS, J. \& KACHELRIESS, M. 2015. Evolution in Computed Tomography: The Battle for Speed and Dose. Investigative Radiology, 50, 629-644.

LEWIS, M., KEAT, N. \& EDYVEAN, S. 2006. Six to ten slice CT scanner comparison report version 14. 06011.

LI, B. T., THOMAS L; HSIEH, JIANG; TANG, XIANGYANG 2012. Simulation and analysis of image quality impacts from single source, ultra-wide coverage CT scanner. Journal of X-Ray Science and Technology, 20, 395-404.

LI, X., ZHANG, D. \& LIU, B. 2013. Monte Carlo assessment of CT dose equilibration in PMMA and water cylinders with diameters from 6 to $55 \mathrm{~cm}$. Medical Physics, 40, 031903.

LOPEZ GONZALES, A. H., TOMAL, A. \& COSTA, P. R. 2015. Evaluation of characteristic-to-total spectrum ratio: Comparison between experimental and a semiempirical model. Applied Radiation and Isotopes, 100, 27-31.

LÜCK, F., KOLDITZ, D., HUPFER, M., STEIDING, C. \& KALENDER, W. A. 2014. Experimental validation of a single shaped filter approach for CT using variable source-to-filter distance for examination of arbitrary object diameters. Physics in Medicine and Biology, 59, 5691-5706.

MAHESH, M. 2009. MDCT Physics: The Basics - Technology, Image Quality and Radiation Dose, Lippincott Williams \& Wilkins.

MARCHAL, G., VOGL, T. J., HEIKEN, J. P. \& RUBIN, G. D. 2005. Multidetector-Row Computed Tomography: Scanning and Contrast Protocols, Springer Milan.

MAXQ. 2019. Available: https://maxq.ai/wp-content/uploads/2019/10/MaxQ-AI-PhilipsAgreement-Release_CLEAN-AND-FINAL_101519.pdf [Accessed 10/11 2019].

MCCOLlOUGH, C. H., LENG, S., YU, L., CODY, D. D., BOONE, J. M. \& MCNITTGRAY, M. F. 2011. CT Dose Index and Patient Dose: They Are Not the Same Thing. Radiology, 259, 311-316.

METROPOLIS, N. \& ULAM, S. 1949. The Monte Carlo Method. Journal of the American Statistical Association, 44, 335-341.

MOLIERE, G. 1948. Theorie der Streuung schneller geladener Teilchen II Mehrfach-und Vielfachstreuung. Zeitschrift für Naturforschung A.

MOORE, M. M., CACAK, R. K. \& HENDEE, W. R. 1981. Multisegmented ion chamber for CT scanner dosimetry. Medical Physics, 8, 640-645. 
MORI, S., ENDO, M., NISHIZAWA, K., TSUNOO, T., AOYAMA, T., FUJIWARA, H. \& MURASE, K. 2005. Enlarged longitudinal dose profiles in cone-beam CT and the need for modified dosimetry. Medical Physics, 32, 1061-1069.

MORNEBURG, H. \& AKTIENGESELLSCHAFT, S. 1995. Bildgebende Systeme für die medizinische Diagnostik: Röntgendiagnostik und Angiographie, Computertomographie, Nuklearmedizin, Magnetresonanztomographie, Sonographie, integrierte Informationssysteme, Publicis MCD Verlag.

OTENDAL, M. 2006. A Compact High-Brightness Liquid-Metal-Jet X-Ray Source Doctoral Thesis, Royal Institute of Technology.

PELC, N. J. 2014. Recent and future directions in CT imaging. Annals of biomedical engineering, 42, 260-268.

PESAPANE, F., CODARI, M. \& SARDANELLI, F. 2018. Artificial intelligence in medical imaging: threat or opportunity? Radiologists again at the forefront of innovation in medicine. European Radiology Experimental, 2, 35.

PUERTO, L. 2018. Monte Carlo Simulation of the Image Acquisition process of a Dual Energy Computed Tomography device Master, University of Sao Paulo.

RADON, J. 1917. "über die Bestimmung von Funktionen durch ihre Integralwerte längs gewisser Mannigfaltigkeiten". Berichte Über Die Verhandlungen der KöniglichSächsischen Akademie der Wissenschaften zu Leipzig, Mathematisch-Physische Klasse [Reports on the Proceedings of the Royal Saxonian Academy of Sciences at Leipzig, Mathematical and Physical Section], 262-277.

RENKER, M., RAMACHANDRA, A., SCHOEPF, U. J., RAUPACH, R., APFALTRER, P., ROWE, G. W., VOGT, S., FLOHR, T. G., KERL, J. M., BAUER, R. W., FINK, C. \& HENZLER, T. 2011. Iterative image reconstruction techniques: Applications for cardiac CT. Journal of Cardiovascular Computed Tomography, 5, 225-230.

ROGERS, D. W. O. 2006. Fifty years of Monte Carlo simulations for medical physics. Physics in Medicine and Biology, 51, R287-R301.

SALVAT, F., FERNÁNDEZ-VAREA, J. \& SEMPAU, J. 2015. PENELOPE: A code system for Monte Carlo simulation of electron and photon transport. In: NEA-OECD. Paris.

SCHARDT, P., DEURINGER, J., FREUDENBERGER, J., HELL, E., KNÜPFER, W., MATTERN, D. \& SCHILD, M. 2004. New x-ray tube performance in computed tomography by introducing the rotating envelope tube technology. Medical Physics, 31, 2699-2706.

SCHINDERA, S. T., ODEDRA, D., RAZA, S. A., KIM, T. K., JANG, H.-J., SZUCSFARKAS, Z. \& ROGALLA, P. 2013. Iterative Reconstruction Algorithm for CT: Can Radiation Dose Be Decreased While Low-Contrast Detectability Is Preserved? Radiology, 269, 511-518.

SCHMIDT, B. \& KALENDER, W. A. 2002. A fast voxel-based Monte Carlo method for scanner- and patient-specific dose calculations in computed tomography. Physica Medica, 18, 43-53.

SCHOEPF, U. J. \& MEINEL, F. G. 2016. Multidetector-Row CT of the Thorax, Springer International Publishing.

SECHOPOULOS, I., ALI, E. S. M., BADAL, A., BADANO, A., BOONE, J. M., KYPRIANOU, I. S., MAINEGRA-HING, E., MCMILLAN, K. L., MCNITT-GRAY, M. F., ROGERS, D. W. O., SAMEI, E. \& TURNER, A. C. 2015. Monte Carlo reference data sets for imaging research: Executive summary of the report of AAPM Research Committee Task Group 195. Medical Physics, 42, 5679-5691.

SEMPAU, J. \& ANDREO, P. 2006. Configuration of the electron transport algorithm of PENELOPE to simulate ion chambers. Physics in Medicine and Biology, 51, 3533. 
SEMPAU, J., BADAL, A. \& BRUALLA, L. 2011. A PENELOPE-based system for the automated Monte Carlo simulation of clinacs and voxelized geometries - application to far-from-axis fields. Medical Physics, 38, 5887-5895.

SEMPAU, J., FERNÁNDEZ-VAREA, J. M., ACOSTA, E. \& SALVAT, F. 2003. Experimental benchmarks of the Monte Carlo code penelope. Nuclear Instruments and Methods in Physics Research Section B: Beam Interactions with Materials and Atoms, 207, 107-123.

SEMPAU, J., SÁNCHEZ-REYES, A., SALVAT, F., TAHAR, H. O. B., JIANG, S. B. \& FERNÁNDEZ-VAREA, J. M. 2001. Monte Carlo simulation of electron beams from an accelerator head using PENELOPE. Physics in Medicine and Biology, 46, 1163.

SHEFER, E., ALTMAN, A., BEHLING, R., GOSHEN, R., GREGORIAN, L., ROTERMAN, Y., UMAN, I., WAINER, N., YAGIL, Y. \& ZARCHIN, O. 2013. State of the Art of CT Detectors and Sources: A Literature Review. Current Radiology Reports, 1, 76-91.

SHOPE, T. B., GAGNE, R. M. \& JOHNSON, G. C. 1981. A method for describing the doses delivered by transmission x-ray computed tomography. Medical Physics, 8, 488-495.

SHOPE, T. B., MORGAN, T. J., SHOWALTER, C. K., PENTLOW, K. S., ROTHENBERG, L. N., WHITE, D. R. \& SPELLER, R. D. 1982. Radiation dosimetry survey of computed tomography systems from ten manufacturers. The British Journal of Radiology, 55, 60-69.

SIEGBAHN, E. A., NILSSON, B., FERNÁNDEZ-VAREA, J. M. \& ANDREO, P. 2003. Calculations of electron fluence correction factors using the Monte Carlo code PENELOPE. Physics in Medicine and Biology, 48, 1263.

SPOKAS, J. J. 1982. Dose descriptors for computed tomography. Medical Physics, 9, 288292.

STERPIN, E., SALVAT, F., CRAVENS, R., RUCHALA, K., OLIVERA, G. H. \& VYNCKIER, S. 2008. Monte Carlo simulation of helical tomotherapy with PENELOPE. Physics in Medicine and Biology, 53, 2161.

SUNG-JOON, Y., IVAN, A. B., PREM, P. \& SHAHID, A. N. 2004. Benchmark of PENELOPE code for low-energy photon transport: dose comparisons with MCNP4 and EGS4. Physics in Medicine and Biology, 49, 387.

SUZUKI, A. \& SUZUKI, M. N. 1978. Use of a pencil-shaped ionization chamber for measurement of exposure resulting from a computed tomography scan. Medical Physics, 5, 536-539.

TEASDALE, E. M. \& AITKEN, S. 2009. Multidetector CT in Neuroimaging: An Atlas and Practical Guide, Clinical Publishing.

TERINI, R., NERSSISSIAN, D. Y., CAMPELO, S. \& YOSHIMURA, E. M. 2017. Caracterização de feixes de TC utilizando Espectrometria Compton. Revista Brasileira de Física Médica, 11, 16-20.

TITUS, F. 1970. Measurements of the energy response functions of scintillators for monoenergetic electrons. Nuclear Instruments and Methods, 89, 93-100.

TSAI, H. Y., TUNG, C. J., HUANG, M. H. \& WAN, Y. L. 2003. Analyses and applications of single scan dose profiles in computed tomography. Medical Physics, 30, 19821989.

TUCKER, D. M., BARNES, G. T. \& CHAKRABORTY, D. P. 1991. Semiempirical model for generating tungsten target X-ray spectra. Med Phys, 18, 211-218.

TURING, A. M. 1937. On Computable Numbers, with an Application to the Entscheidungsproblem. Proceedings of the London Mathematical Society, s2-42, 230265. 
TURNER, A. C., ZHANG, D., KHATONABADI, M., ZANKL, M., DEMARCO, J. J., CAGNON, C. H., CODY, D. D., STEVENS, D. M., MCCOLlOUGH, C. H. \& MCNITT-GRAY, M. F. 2011. The feasibility of patient size-corrected, scannerindependent organ dose estimates for abdominal CT exams. Medical physics, 38, 820829.

ULZHEIMER, S. \& FLOHR, T. 2009. Multislice CT: Current Technology and Future Developments. In: REISER, M. F., BECKER, C. R., NIKOLAOU, K. \& GLAZER, G. (eds.) Multislice CT. Berlin, Heidelberg: Springer Berlin Heidelberg.

UMISEDO, N. K. \& YOSHIMURA, E. M. POSLI - Instrumentação para leitura de detetores OSL em forma de fita. XXII CONGRESSO BRASILEIRO DE FÍSICA MÉDICA, 2017 Ribeirão Preto - SP. ABFM.

WILSON, R. R. 1952. Monte Carlo Study of Shower Production. Physical Review, 86, 261269.

WOOD, R. W. 1896. Note on "focus tubes" for producing x-rays. The London, Edinburgh, and Dublin Philosophical Magazine and Journal of Science, 41, 382-383.

YUKIHARA, E. G. \& MCKEEVER, S. W. S. 2008. Optically stimulated luminescence (OSL) dosimetry in medicine. Physics in Medicine and Biology, 53, R351-R379.

ZENG, G. 2010. Medical Image Reconstruction: A Conceptual Tutorial, Springer Berlin Heidelberg.

ZHANG, D., SAVANDI, A. S., DEMARCO, J. J., CAGNON, C. H., ANGEL, E., TURNER, A. C., CODY, D. D., STEVENS, D. M., PRIMAK, A. N., MCCOLlOUGH, C. H. \& MCNITT-GRAY, M. F. 2009. Variability of surface and center position radiation dose in MDCT: Monte Carlo simulations using CTDI and anthropomorphic phantoms. Medical Physics, 36, 1025-1038.

ZHENG-MING, L. \& BRAHME, A. 1993. An overview of the transport theory of charged particles. Radiation Physics and Chemistry, 41, 673-703. 